Supporting Information

\title{
Asymmetric $\alpha$-Pentadienylation of Aldehydes with Cyclopropylacetylenes
}

$$
\text { Min-Song } \mathrm{Wu}^{\dagger}, \text { Zhi-Yong Han*† and Liu-Zhu Gong*†: }
$$

${ }^{\dagger}$ Hefei National Laboratory for Physical Sciences at the Microscale and Department of Chemistry, University of Science and Technology of China, Hefei, 230026, China

$\$$ Collaborative Innovation Center of Chemical Science and Engineering (Tianjin), Tianjin, 300072, China E-mail: hanzy2014@ustc.edu.cn;gonglz@ustc.edu.cn

\section{Table of Contents}

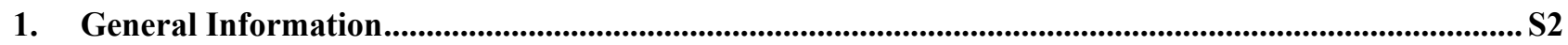

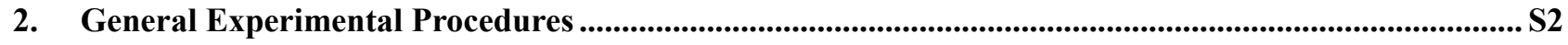

3. Characterization Data for the Product and Absolute Configuration Determination ............................... S5

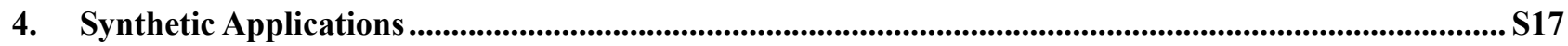

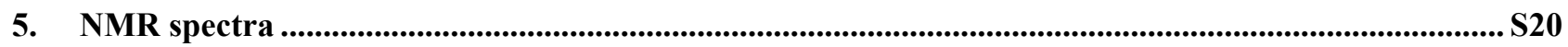

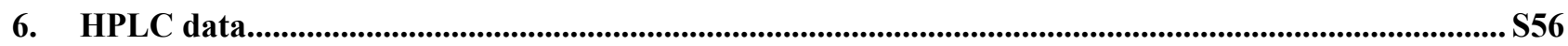




\section{General Information}

NMR spectra were recorded on a Brucker-400 MHz spectrometer and a Brucker-500 $\mathrm{MHz}$ spectrometer. Chemical shifts $(\delta)$ are given in ppm relative to TMS. The residual solvent signals were used as references and the chemical shifts converted to the TMS scale $\left(\mathrm{CDCl}_{3}: \delta \mathrm{H}=7.26 \mathrm{ppm}, \delta \mathrm{C}=\right.$ $77.16 \mathrm{ppm}$ ). HPLC analysis was performed on Waters-Breeze (2998 PDADetector and e2695 Separations Module, UV detection monitored at 210-254nm). Chiralpak AS, OD, IB, IC, ID, OJ columns were purchased from Daicel Chemical Industries, Ltd. Optical rotationswere determined at $589 \mathrm{~nm}$ (sodium D line) by using a Perkin-Elmer-343 polarimeter. The high resolution mass spectra were recorded on a Thermo LTQ Orbitrap XL (ESI+). Infrared spectra were recorded on a Nicolet MX-1E FT-IR spectrometer.

Starting materials were purchased from commercial suppliers (Alfa, TCI, Energy, etc.) and used as supplied unless otherwise stated. $\mathrm{Pd}\left(\mathrm{PPh}_{3}\right)_{4}$ was purchased from TCI. The ligands and acids were purchased from TCI or Daicel Chemical Industries, Ltd. The substituted cyclopropylacetylenes ${ }^{1-2}$ and aldehyde ${ }^{3-5}$ were known compounds and prepared by following the literature report.

\section{General Experimental Procedures}

\section{Method A:}

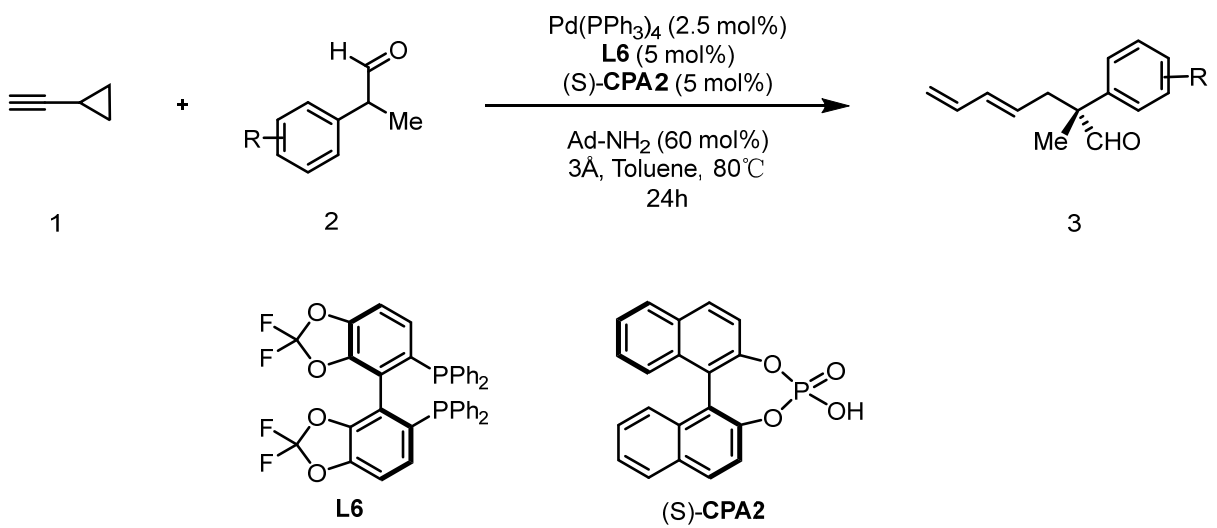

To a flame-dried and $\mathrm{N}_{2}$-purged Schlenk tube $(10 \mathrm{~mL})$ were added $\mathrm{Pd}\left(\mathrm{PPh}_{3}\right)_{4}(0.005 \mathrm{mmol}, 5.8 \mathrm{mg})$, L6 $(0.01 \mathrm{mmol}, 6.8 \mathrm{mg})$, (S)-CPA2 $(0.01 \mathrm{mmol}, 3.5 \mathrm{mg})$, Ad-NH $2(0.12 \mathrm{mmol}, 18.2 \mathrm{mg})$, and $3 \AA$ MS $(60.0 \mathrm{mg})$. The Schlenk tube was then evacuated and filled with $\mathrm{N}_{2}$. This cycle was repeated three times and followed by addition of aldehyde $(0.2 \mathrm{mmol})$, which was dissolved in toluene $(1 \mathrm{~mL})$, and then cyclopropylacetylene $(1.0 \mathrm{mmol}, 66.0 \mathrm{mg})$ was added via syringe. The mixture was stirred at $80^{\circ} \mathrm{C}$ for $24 \mathrm{~h}$. After the vial was cooled down to room temperature, the reaction mixture was acidified with $2 \mathrm{~N} \mathrm{HCl}(2 \mathrm{~mL})$ at room temperature for 30 minutes and extracted with ethyl ether $(4 \times 5 \mathrm{~mL})$. The combined organic layers were dried over anhydrous $\mathrm{Na}_{2} \mathrm{SO}_{4}$. The mixture was filtered and concentrated in vacuo. The residue was purified by flash chromatography $\left(\mathrm{SiO}_{2}\right.$, hexanes/ ethyl ether $=20 / 1$ to $15 / 1)$ to provide the $\alpha$-pentadienylation product 3 . 


\section{Method B:}
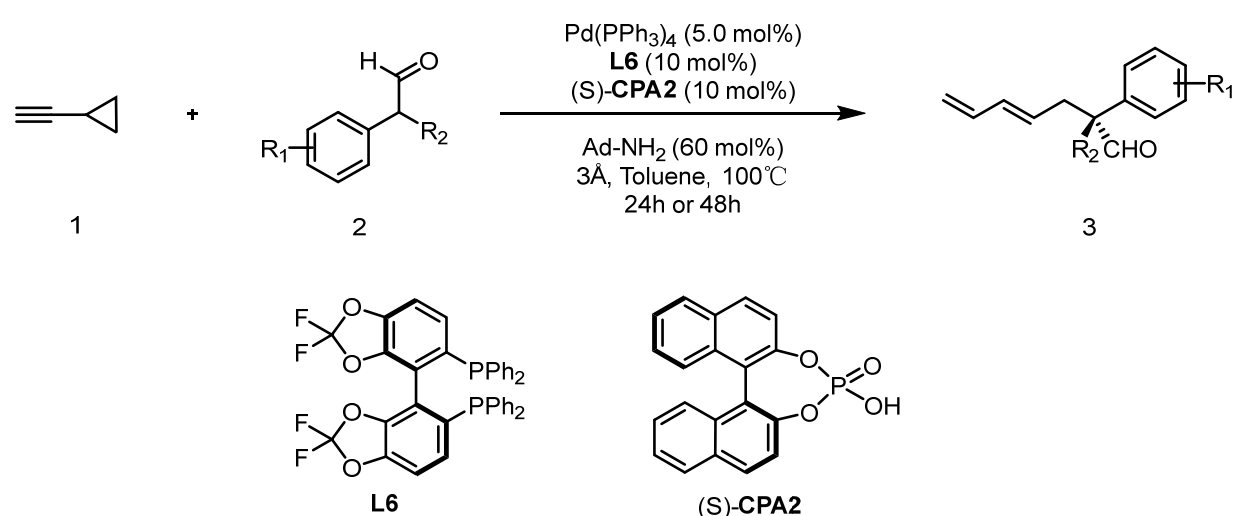

(S)-CPA2

To a flame-dried and $\mathrm{N}_{2}$-purged Schlenk tube $(10 \mathrm{~mL})$ were added $\mathrm{Pd}\left(\mathrm{PPh}_{3}\right)_{4}(0.01 \mathrm{mmol}, 11.6 \mathrm{mg})$, L6 (0.02 mmol, $13.6 \mathrm{mg})$, (S)-CPA2 (0.02 mmol, $7.0 \mathrm{mg})$, Ad-NH $2(0.12 \mathrm{mmol}, 18.2 \mathrm{mg})$, and $3 \AA$ MS $(60.0 \mathrm{mg})$. The Schlenk tube was then evacuated and filled with $\mathrm{N}_{2}$. This cycle was repeated three times and followed by addition of addition of aldehyde $(0.2 \mathrm{mmol})$, which was dissolved in toluene $(1.0 \mathrm{~mL})$, and then cyclopropylacetylene $(1.0 \mathrm{mmol}, 66 \mathrm{mg})$ was added via syringe. The mixture was stirred at $100{ }^{\circ} \mathrm{C}$ for $24 \mathrm{~h}$ or $48 \mathrm{~h}$. After the vial was cooled down to room temperature, the reaction mixture was acidified with $2 \mathrm{~N} \mathrm{HCl}(2 \mathrm{~mL})$ at room temperature for 30 minutes and extracted with ethyl ether $(4 \times 5 \mathrm{~mL})$. The combined organic layers were dried over anhydrous $\mathrm{Na}_{2} \mathrm{SO}_{4}$. The mixture was filtered and concentrated in vacuo. The residue was purified by flash chromatography $\left(\mathrm{SiO}_{2}\right.$, hexanes/ ethyl ether $=20 / 1$ to $15 / 1$ ) to provide the $\alpha$-pentadienylation product 3 .

\section{Method C:}

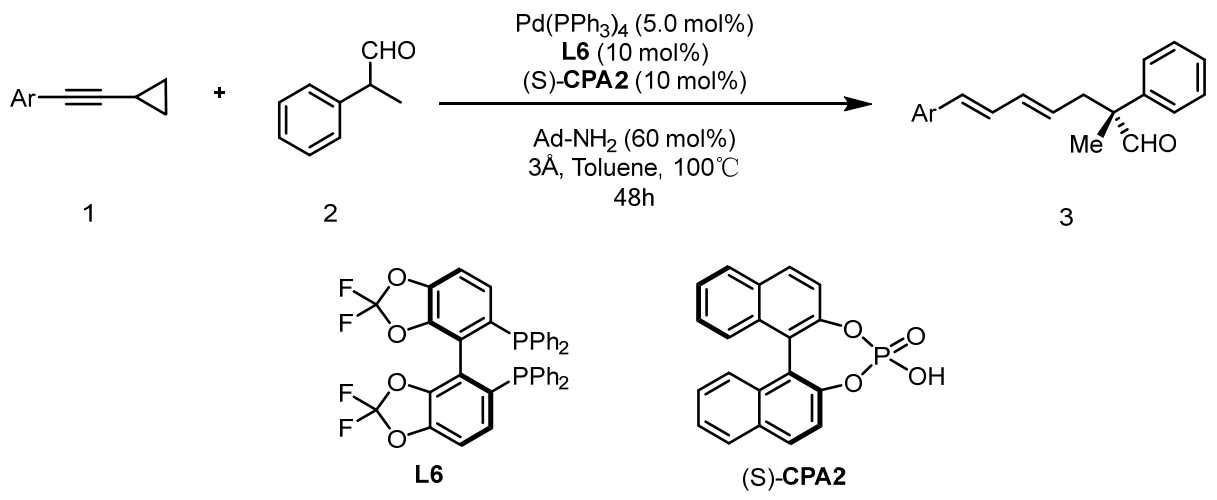

To a flame-dried and $\mathrm{N}_{2}$-purged Schlenk tube $(10 \mathrm{~mL})$ were added $\mathrm{Pd}\left(\mathrm{PPh}_{3}\right)_{4}(0.01 \mathrm{mmol}, 11.6 \mathrm{mg})$, L6 (0.02 mmol, $13.6 \mathrm{mg})$, (S)-CPA2 (0.02 mmol, $7.0 \mathrm{mg}), \mathrm{Ad}_{-} \mathrm{NH}_{2}(0.12 \mathrm{mmol}, 18.2 \mathrm{mg})$, and $3 \AA$ MS $(60.0 \mathrm{mg})$. The schlenk tube was then evacuated and filled with $\mathrm{N}_{2}$. This cycle was repeated three times and followed by addition of addition of aldehyde $(0.2 \mathrm{mmol}, 26.8 \mathrm{mg})$, which was dissolved in toluene $(1.0 \mathrm{~mL})$, and then cyclopropylacetylene $(0.4 \mathrm{mmol}$ or $1.0 \mathrm{mmol})$ was added via syringe. The mixture was stirred at $100{ }^{\circ} \mathrm{C}$ for $48 \mathrm{~h}$. After the vial was cooled down to room temperature, the reaction mixture was acidified with $2 \mathrm{~N} \mathrm{HCl}(2 \mathrm{~mL})$ at room temperature for 30 minutes and extracted with ethyl ether $(4 \times 5 \mathrm{~mL})$. The combined organic layers were dried over anhydrous $\mathrm{Na}_{2} \mathrm{SO}_{4}$. The mixture was filtered and concentrated in vacuo. The residue was purified by flash chromatography $\left(\mathrm{SiO}_{2}\right.$, hexanes/ ethyl ether $=20 / 1$ to $\left.15 / 1\right)$ to provide the $\alpha$-pentadienylation product 3 . 
Method D:

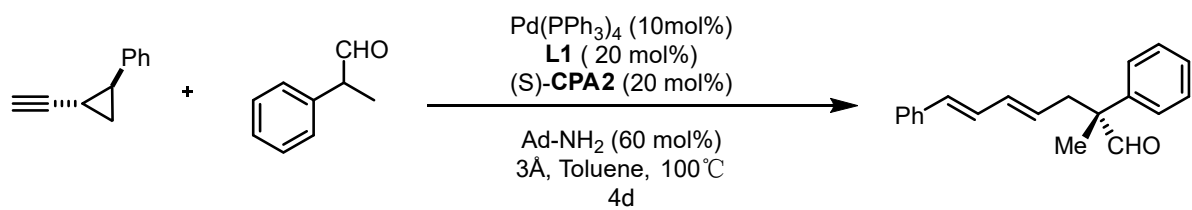

(土)-trans-1n

2a

(E)-3r

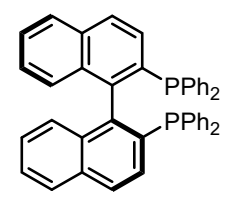

L1

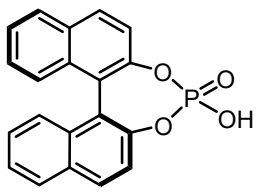

(S)-CPA2

To a flame-dried and $\mathrm{N}_{2}$-purged Schlenk tube $(10 \mathrm{~mL})$ were added $\mathrm{Pd}\left(\mathrm{PPh}_{3}\right)_{4}(0.02 \mathrm{mmol}, 23.2 \mathrm{mg})$, L1 (0.04 mmol, $24.8 \mathrm{mg})$, (S)-CPA2 (0.04 mmol, $14.0 \mathrm{mg}), \mathrm{Ad}-\mathrm{NH}_{2}(0.12 \mathrm{mmol}, 18.2 \mathrm{mg})$, and $3 \AA$ MS (60.0 mg). The Schlenk tube was then evacuated and filled with $\mathrm{N}_{2}$. This cycle was repeated three times and followed by addition of addition of $\mathbf{2 a}(0.2 \mathrm{mmol}, 26.8 \mathrm{mg})$, which was dissolved in toluene $(1.0 \mathrm{~mL})$, and then $( \pm)$-trans $-\mathbf{1 n}(1.0 \mathrm{mmol}, 142.0 \mathrm{mg})$ was added via syringe. The mixture was stirred at $100{ }^{\circ} \mathrm{C}$ for $96 \mathrm{~h}$. After the vial was cooled down to room temperature, the reaction mixture was acidified with $2 \mathrm{~N} \mathrm{HCl}(2 \mathrm{~mL})$ at room temperature for 30 minutes and extracted with ethyl ether (4 $\times 5 \mathrm{~mL}$ ). The combined organic layers were dried over anhydrous $\mathrm{Na}_{2} \mathrm{SO}_{4}$. The mixture was filtered and concentrated in vacuo. The residue was purified by flash chromatography $\left(\mathrm{SiO}_{2}\right.$, hexanes/ ethyl ether $=20 / 1)$ to provide the $\alpha$-pentadienylation product $(E)-3 \mathbf{r}$ as a yellowish oil. Yield: $28.2 \mathrm{mg}$, $51 \%$; E/Z $>20 / 1$; ee $=82 \%$, determined by HPLC (OD, hexane/isopropanol $=97 / 3$, flow rate 1.0 $\mathrm{mL} / \mathrm{min}, \mathrm{T}=30{ }^{\circ} \mathrm{C}, 254 \mathrm{~nm}$ ): $\mathrm{t}_{\mathrm{R}}=9.512 \mathrm{~min}$ (major), $\mathrm{t}_{\mathrm{R}}=11.040 \mathrm{~min}$ (minor). $[\alpha]_{\mathrm{d}}{ }^{20}=-88.0$ (c 1.30 , $\left.\mathrm{CH}_{2} \mathrm{Cl}_{2}\right)$,

${ }^{1}$ H NMR (500 MHz, Chloroform-d) $\delta 9.47(\mathrm{~s}, 1 \mathrm{H}), 7.35-7.31(\mathrm{~m}, 2 \mathrm{H}), 7.28-7.19(\mathrm{~m}, 7 \mathrm{H}), 7.14-$ $7.10(\mathrm{~m}, 1 \mathrm{H}), 6.59(\mathrm{dd}, J=15.7,10.4 \mathrm{~Hz}, 1 \mathrm{H}), 6.36(\mathrm{~d}, J=15.7 \mathrm{~Hz}, 1 \mathrm{H}), 6.15(\mathrm{dd}, J=15.0,10.4 \mathrm{~Hz}$, $1 \mathrm{H}), 5.46(\mathrm{dt}, J=15.1,7.6 \mathrm{~Hz}, 1 \mathrm{H}), 2.73-2.62(\mathrm{~m}, 2 \mathrm{H}), 1.40(\mathrm{~s}, 3 \mathrm{H})$.

${ }^{13}$ C NMR (126 MHz, Chloroform-d) $\delta$ 202.1, 139.5, 137.4, 134.3, 131.3, 129.4, 129.0, 128.8, 128.7, $127.5,127.5,127.3,126.3,54.2,39.9,19.1$.

HRMS (ESI-TOF) m/z: [M+H] $]^{+}$Calcd for $\mathrm{C}_{20} \mathrm{H}_{21} \mathrm{O}$ 277.1587; Found 277.1584.

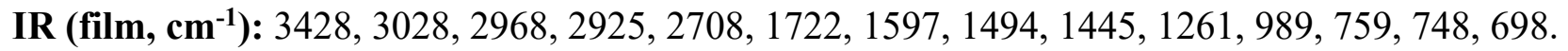

\section{Method of 2 mmol scale:}

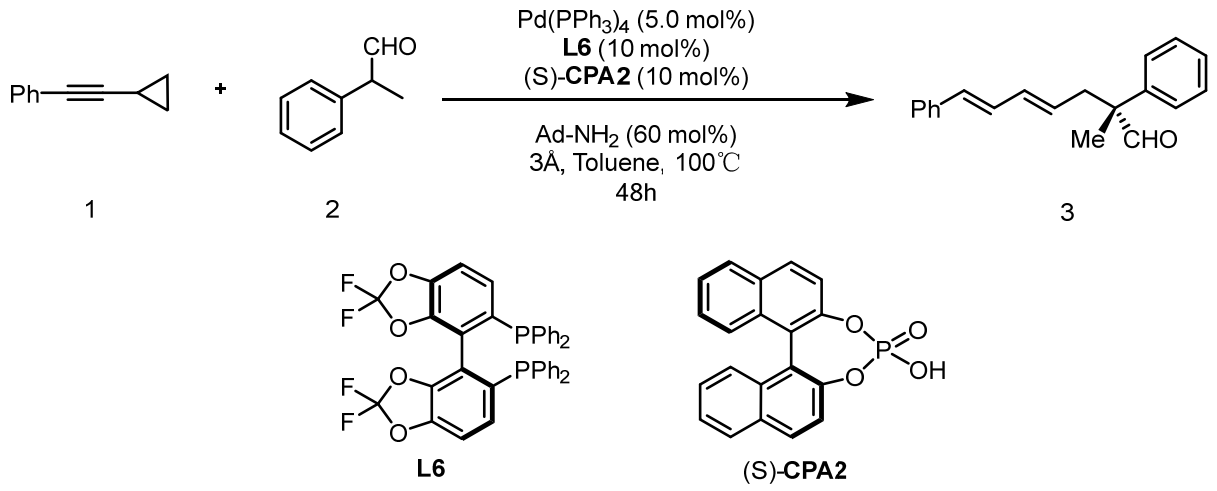

To a flame-dried and $\mathrm{N}_{2}$-purged Schlenk tube $(100 \mathrm{~mL})$ were added $\mathrm{Pd}\left(\mathrm{PPh}_{3}\right)_{4}(0.1 \mathrm{mmol}, 116.0 \mathrm{mg})$, L6 (0.2 mmol, $136.4 \mathrm{mg}),(\mathrm{S})$-CPA2 (0.2 mmol, $69.6 \mathrm{mg}), \mathrm{Ad}_{-} \mathrm{NH}_{2}(1.2 \mathrm{mmol}, 181.4 \mathrm{mg})$, and $3 \AA$ 
MS (600 mg). The schlenk tube was then evacuated and filled with $\mathrm{N}_{2}$. This cycle was repeated three times and followed by addition of addition of aldehyde $(2.0 \mathrm{mmol}, 268.1 \mathrm{mg})$, which was dissolved in toluene $(10 \mathrm{~mL})$, and then cyclopropylacetylene $(4.0 \mathrm{mmol}, 571.0 \mathrm{mg})$ was added via syringe. The mixture was stirred at $100{ }^{\circ} \mathrm{C}$ for $48 \mathrm{~h}$. After the vial was cooled down to room temperature, the reaction mixture was acidified with $2 \mathrm{~N} \mathrm{HCl}(10 \mathrm{~mL})$ at room temperature for 30 minutes and extracted with ethyl ether $(4 \times 20 \mathrm{~mL})$. The combined organic layers were dried over anhydrous $\mathrm{Na}_{2} \mathrm{SO}_{4}$. The mixture was filtered and concentrated in vacuo. The residue was purified by flash chromatography $\left(\mathrm{SiO}_{2}\right.$, hexanes/ ethyl ether $\left.=15 / 1\right)$ to provide a yellowish oil, yield: $497.1 \mathrm{mg}, 90 \%$; ee $=89 \%$.

\section{Characterization Data for the Product and Absolute Configuration}

\section{Determination}

(R,E)-2-methyl-2-phenylhepta-4,6-dienal (3a). $0.2 \mathrm{mmol}$ aldehyde and $1.0 \mathrm{mmol}$

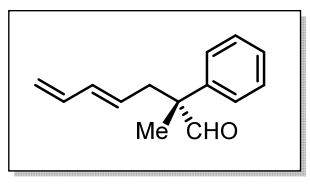
cyclopropylacetylene reacted in the presence of $\mathrm{Pd}\left(\mathrm{PPh}_{3}\right)_{4}(0.005 \mathrm{mmol}), \mathbf{L 6}$ $(0.01 \mathrm{mmol}), \mathbf{A 2}(0.12 \mathrm{mmol})$ and $(\mathrm{S})$-CPA2 $(0.01 \mathrm{mmol})$ and $3 \AA$ molecular sieves $(60 \mathrm{mg})$ in toluene $(1.0 \mathrm{~mL})$ at $80{ }^{\circ} \mathrm{C}$ for $24 \mathrm{~h}$ following the method $\mathrm{A}$. Flash chromatography $\left(\mathrm{SiO}_{2}\right.$, hexanes/ ethyl ether $\left.=20 / 1\right)$ of crude product gave pure 3a as a light yellow oil. Yield: $31.2 \mathrm{mg}, 78 \%$; E/Z $>20 / 1$; L/B $>20 / 1$; ee $=90 \%$, determined by HPLC (AS, hexane/isopropanol $=95 / 5$, flow rate $0.5 \mathrm{~mL} / \mathrm{min}, \mathrm{T}=30{ }^{\circ} \mathrm{C}, 220 \mathrm{~nm}$ ): $\mathrm{t}_{\mathrm{R}}=9.510 \mathrm{~min}$ (major), $\mathrm{t}_{\mathrm{R}}=10.266 \mathrm{~min}$ (minor). $[\alpha]_{\mathrm{d}}{ }^{20}=-65.5\left(\mathrm{c} 0.60, \mathrm{CH}_{2} \mathrm{Cl}_{2}\right.$ ).

${ }^{1}$ H NMR (500 MHz, Chloroform-d) $\delta 9.48(\mathrm{~s}, 1 \mathrm{H}), 7.38-7.33(\mathrm{~m}, 2 \mathrm{H}), 7.26(\mathrm{~m}, 1 \mathrm{H}), 7.21(\mathrm{~m}, 2 \mathrm{H})$, $6.17(\mathrm{dt}, J=16.9,10.2 \mathrm{~Hz}, 1 \mathrm{H}), 6.02(\mathrm{dd}, J=15.1,10.4 \mathrm{~Hz}, 1 \mathrm{H}), 5.38(\mathrm{dt}, J=15.0,7.5 \mathrm{~Hz}, 1 \mathrm{H}), 5.05$ $(\mathrm{d}, J=16.9 \mathrm{~Hz}, 1 \mathrm{H}), 4.93(\mathrm{~d}, J=10.1 \mathrm{~Hz}, 1 \mathrm{H}), 2.65(\mathrm{~m}, 2 \mathrm{H}), 1.40(\mathrm{~s}, 3 \mathrm{H})$.

${ }^{13}$ C NMR (126 MHz, Chloroform-d) $\delta$ 202.1, 139.2, 136.8, 134.8, 129.2, 129.0, 127.5, 127.3, 116.2, $54.1,39.5,19.1$.

HRMS (ESI-TOF) m/z: [M+H] $]^{+}$Calcd for $\mathrm{C}_{14} \mathrm{H}_{17} \mathrm{O}$ 201.1274; Found 201.1279.

IR (film, $\mathbf{c m}^{-1}$ ): 3428, 3086, 2972, 2931, 2807, 2709, 1724, 1600, 1495, 1445, 1005, 902, 761, 699.

(R,E)-2-methyl-2-(p-tolyl)hepta-4,6-dienal (3b). $0.2 \quad \mathrm{mmol}$ aldehyde and 1.0 mmol

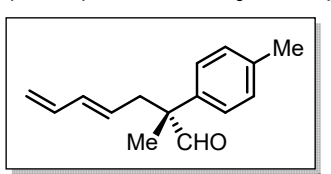

cyclopropylacetylene reacted in the presence of $\mathrm{Pd}\left(\mathrm{PPh}_{3}\right)_{4}(0.005 \mathrm{mmol}), \mathbf{L 6}$ (0.01 mmol), A2 $(0.12 \mathrm{mmol})$ and $(\mathrm{S})$-CPA2 $(0.01 \mathrm{mmol})$ and $3 \AA$ molecular sieves $(60 \mathrm{mg})$ in toluene $(1.0 \mathrm{~mL})$ at $80{ }^{\circ} \mathrm{C}$ for $24 \mathrm{~h}$ following the method $\mathrm{A}$. Flash chromatography $\left(\mathrm{SiO}_{2}\right.$, hexanes/ ethyl ether $\left.=20 / 1\right)$ of crude product gave pure $\mathbf{3 b}$ as a light yellow oil. Yield: $30.0 \mathrm{mg}, 70 \%$; $\mathrm{E} / \mathrm{Z}>20 / 1 ; \mathrm{L} / \mathrm{B}>20 / 1$; ee $=93 \%$, determined by HPLC (AS, hexane/isopropanol $=95 / 5$, flow rate $0.5 \mathrm{~mL} / \mathrm{min}, \mathrm{T}=30{ }^{\circ} \mathrm{C}, 220 \mathrm{~nm}$ ): $\mathrm{t}_{\mathrm{R}}=9.407 \mathrm{~min}$ (major), $\mathrm{t}_{\mathrm{R}}=10.544 \mathrm{~min}$ (minor). $[\alpha]_{\mathrm{d}}{ }^{20}=-120.3\left(\mathrm{c} 0.93, \mathrm{CH}_{2} \mathrm{Cl}_{2}\right)$.

${ }^{1}$ H NMR (500 MHz, Chloroform-d) $\delta 9.49(\mathrm{~s}, 1 \mathrm{H}), 7.20(\mathrm{~d}, 2 \mathrm{H}), 7.14(\mathrm{~d}, 2 \mathrm{H}), 6.22(\mathrm{dt}, J=16.9,10.3$ $\mathrm{Hz}, 1 \mathrm{H}), 6.07(\mathrm{dd}, J=15.1,10.4 \mathrm{~Hz}, 1 \mathrm{H}), 5.43(\mathrm{dt}, J=15.1,7.5 \mathrm{~Hz}, 1 \mathrm{H}), 5.10(\mathrm{~d}, J=16.9 \mathrm{~Hz}, 1 \mathrm{H})$, $4.98(\mathrm{~d}, J=10.1 \mathrm{~Hz}, 1 \mathrm{H}), 2.74-2.61(\mathrm{~m}, 2 \mathrm{H}), 2.35(\mathrm{~s}, 3 \mathrm{H}), 1.42(\mathrm{~s}, 3 \mathrm{H})$.

${ }^{13}$ C NMR (126 MHz, Chloroform-d) $\delta$ 202.1, 137.3, 136.8, 136.4, 134.7, 129.7, 129.3, 127.2, 116.1, 53.7, 39.5, 21.1, 19.1.

HRMS (ESI-TOF) m/z: [M+H] $]^{+}$Calcd for $\mathrm{C}_{15} \mathrm{H}_{19} \mathrm{O}$ 215.1430; Found 215.1429.

IR (film, $\mathbf{c m}^{-1}$ ): 3088, 2970, 2922, 2705, 1725, 1513, 1004, 901, 812, 721, 536. 
(R,E)-2-(4-methoxyphenyl)-2-methylhepta-4,6-dienal (3c). $0.2 \mathrm{mmol}$ aldehyde and $1.0 \mathrm{mmol}$

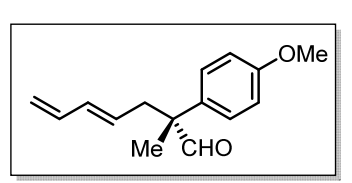
cyclopropylacetylene reacted in the presence of $\mathrm{Pd}\left(\mathrm{PPh}_{3}\right)_{4}(0.005 \mathrm{mmol}), \mathbf{L 6}$ (0.01 mmol), A2 $(0.12 \mathrm{mmol})$ and (S)-CPA2 $(0.01 \mathrm{mmol})$ and $3 \AA$ molecular sieves $(60 \mathrm{mg})$ in toluene $(1.0 \mathrm{~mL})$ at $80{ }^{\circ} \mathrm{C}$ for $24 \mathrm{~h}$ following the method $\mathrm{A}$. Flash chromatography $\left(\mathrm{SiO}_{2}\right.$, hexanes/ ethyl ether $\left.=15 / 1\right)$ of crude product gave pure 3c as a light yellow oil. Yield: $27.2 \mathrm{mg}, 59 \%$; $\mathrm{E} / \mathrm{Z}>20 / 1 ; \mathrm{L} / \mathrm{B}>20 / 1$; ee $=93 \%$, determined by HPLC (AS, hexane/isopropanol $=95 / 5$, flow rate $0.5 \mathrm{~mL} / \mathrm{min}, \mathrm{T}=30{ }^{\circ} \mathrm{C}, 220 \mathrm{~nm}$ ): $\mathrm{t}_{\mathrm{R}}=14.135$ $\min$ (major), $\mathrm{t}_{\mathrm{R}}=15.933 \mathrm{~min}$ (minor). $[\alpha]_{\mathrm{d}}{ }^{20}=-75.1\left(\mathrm{c} 0.87, \mathrm{CH}_{2} \mathrm{Cl}_{2}\right)$.

${ }^{1}$ H NMR $(500 \mathrm{MHz}$, Chloroform- $d) \delta 9.46(\mathrm{~s}, 1 \mathrm{H}), 7.17(\mathrm{~d}, J=8.9 \mathrm{~Hz}, 2 \mathrm{H}), 6.92(\mathrm{~d}, J=8.9 \mathrm{~Hz}, 2 \mathrm{H})$, 6.22 (dt, $J=16.9,10.3 \mathrm{~Hz}, 1 \mathrm{H}), 6.07$ (dd, $J=15.1,10.4 \mathrm{~Hz}, 1 \mathrm{H}), 5.42(\mathrm{dt}, J=15.0,7.5 \mathrm{~Hz}, 1 \mathrm{H}), 5.09$ (d, $J=16.9 \mathrm{~Hz}, 1 \mathrm{H}), 4.97$ (d, $J=10.1 \mathrm{~Hz}, 1 \mathrm{H}), 3.81(\mathrm{~s}, 3 \mathrm{H}), 2.72-2.59(\mathrm{~m}, 2 \mathrm{H}), 1.41(\mathrm{~s}, 3 \mathrm{H})$.

${ }^{13}$ C NMR (126 MHz, Chloroform-d) $\delta$ 201.9, 158.9, 136.8, 134.6, 131.2, 129.3, 128.4, 116.1, 114.4, $55.4,53.4,39.5,19.1$.

HRMS (ESI-TOF) m/z: [M+H] $]^{+}$Calcd for $\mathrm{C}_{15} \mathrm{H}_{19} \mathrm{O}_{2} 231.1380$; Found 231.1382.

IR (film, $\mathbf{c m}^{-1}$ ): 3084, 2963, 2931, 2836, 2707, 1720, 1608, 1513, 1256, 1184, 1032, 827, 797, 550.

(R,E)-2-(4-fluorophenyl)-2-methylhepta-4,6-dienal (3d). $0.2 \mathrm{mmol}$ aldehyde and $1.0 \mathrm{mmol}$

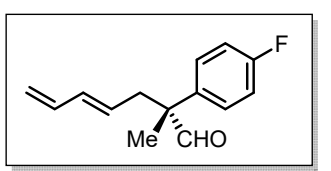
cyclopropylacetylene reacted in the presence of $\mathrm{Pd}\left(\mathrm{PPh}_{3}\right)_{4}(0.005 \mathrm{mmol}), \mathbf{L 6}$ (0.01 mmol), A2 (0.12 mmol) and (S)-CPA2 (0.01 mmol) and $3 \AA$ molecular sieves $(60 \mathrm{mg})$ in toluene $(1.0 \mathrm{~mL})$ at $80{ }^{\circ} \mathrm{C}$ for $24 \mathrm{~h}$ following the method $\mathrm{A}$. Flash chromatography $\left(\mathrm{SiO}_{2}\right.$, hexanes/ ethyl ether $\left.=20 / 1\right)$ of crude product gave pure 3d as a light yellow oil. Yield: $26.2 \mathrm{mg}, 60 \%$; $\mathrm{E} / \mathrm{Z}>20 / 1$; L/B $>20 / 1$; ee $=87 \%$, determined by HPLC (AS, hexane/isopropanol $=95 / 5$, flow rate $0.5 \mathrm{~mL} / \mathrm{min}, \mathrm{T}=30{ }^{\circ} \mathrm{C}, 220 \mathrm{~nm}$ ): $\mathrm{t}_{\mathrm{R}}=10.637 \mathrm{~min}$ (major), $\mathrm{t}_{\mathrm{R}}=11.302 \mathrm{~min}$ (minor). $[\alpha]_{\mathrm{d}}{ }^{20}=-79.4\left(\mathrm{c} 1.18, \mathrm{CH}_{2} \mathrm{Cl}_{2}\right.$ ).

${ }^{1}$ H NMR (400 MHz, Chloroform-d) $\delta 9.49(\mathrm{~s}, 1 \mathrm{H}), 7.25-7.17(\mathrm{~m}, 2 \mathrm{H}), 7.12-7.03$ (m, 2H), 6.21 (dt, $J=16.9,10.2 \mathrm{~Hz}, 1 \mathrm{H}), 6.05$ (dd, $J=15.0,10.5 \mathrm{~Hz}, 1 \mathrm{H}), 5.39$ (dt, $J=15.0,7.5 \mathrm{~Hz}, 1 \mathrm{H}), 5.09$ (d, $J=16.7 \mathrm{~Hz}, 1 \mathrm{H}), 4.98(\mathrm{~d}, J=10.0 \mathrm{~Hz}, 1 \mathrm{H}), 2.74-2.57(\mathrm{~m}, 2 \mathrm{H}), 1.43(\mathrm{~s}, 3 \mathrm{H})$.

${ }^{13} \mathrm{C}$ NMR (126 MHz, Chloroform- $\left.d\right) \delta 201.7,163.1\left(\mathrm{C}-\mathrm{F},{ }^{1} \mathrm{~J}_{\mathrm{C}-\mathrm{F}}=246.8 \mathrm{~Hz}\right), 161.2\left(\mathrm{C}-\mathrm{F},{ }^{1} \mathrm{~J}_{\mathrm{C}-\mathrm{F}}=246.8\right.$ $\mathrm{Hz}), 136.7,135.2\left(\mathrm{C}-\mathrm{F},{ }^{4} \mathrm{~J}_{\mathrm{C}-\mathrm{F}}=3.7 \mathrm{~Hz}\right), 135.2\left(\mathrm{C}-\mathrm{F},{ }^{4} \mathrm{~J}_{\mathrm{C}-\mathrm{F}}=3.7 \mathrm{~Hz}\right), 135.0,129.0\left(\mathrm{C}-\mathrm{F},{ }^{3} \mathrm{~J}_{\mathrm{C}-\mathrm{F}}=8.7 \mathrm{~Hz}\right)$, $129.0\left(\mathrm{C}-\mathrm{F},{ }^{3} \mathrm{~J}_{\mathrm{C}-\mathrm{F}}=8.7 \mathrm{~Hz}\right), 128.7,116.4,116.0\left(\mathrm{C}-\mathrm{F},{ }^{2} \mathrm{~J}_{\mathrm{C}-\mathrm{F}}=21.1 \mathrm{~Hz}\right), 115.8\left(\mathrm{C}-\mathrm{F},{ }^{2} \mathrm{~J}_{\mathrm{C}-\mathrm{F}}=21.1 \mathrm{~Hz}\right)$, 53.6, 39.7, 19.2.

${ }^{19}$ F NMR (376 MHz, Chloroform-d) $\delta-115.1$.

HRMS (ESI-TOF) m/z: [M+H] $]^{+}$Calcd for $\mathrm{C}_{14} \mathrm{H}_{16} \mathrm{FO} 219.1180$; Found 219.1182.

IR (film, $\mathbf{c m}^{-1}$ ): 3085, 2972, 2918, 2849, 2709, 1726, 1602, 1510, 1236, 1166, 1005, 905, 832, 541.

(R,E)-2-(3-chlorophenyl)-2-methylhepta-4,6-dienal (3e). $0.2 \mathrm{mmol}$ aldehyde and $1.0 \mathrm{mmol}$

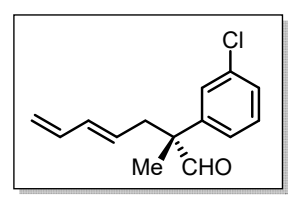
cyclopropylacetylene reacted in the presence of $\mathrm{Pd}\left(\mathrm{PPh}_{3}\right)_{4}(0.005 \mathrm{mmol}), \mathbf{L 6}(0.01$ $\mathrm{mmol}), \mathbf{A 2}(0.12 \mathrm{mmol})$ and (S)-CPA2 $(0.01 \mathrm{mmol})$ and $3 \AA$ molecular sieves $(60$ $\mathrm{mg})$ in toluene $(1.0 \mathrm{~mL})$ at $80{ }^{\circ} \mathrm{C}$ for $24 \mathrm{~h}$ following the method A. Flash chromatography $\left(\mathrm{SiO}_{2}\right.$, hexanes/ ethyl ether $\left.=20 / 1\right)$ of crude product gave pure 3e as a light yellow oil. Yield: $35.1 \mathrm{mg}, 75 \%$; E/Z $>20 / 1 ; \mathrm{L} / \mathrm{B}>20 / 1$; ee $=84 \%$, determined by HPLC (AS, hexane/isopropanol $=95 / 5$, flow rate $0.5 \mathrm{~mL} / \mathrm{min}, \mathrm{T}=30^{\circ} \mathrm{C}, 220 \mathrm{~nm}$ ): $\mathrm{t}_{\mathrm{R}}=9.707 \mathrm{~min}$ (major), $\mathrm{t}_{\mathrm{R}}=10.244 \min$ (minor). $[\alpha]_{\mathrm{d}}{ }^{20}=-79.4\left(\mathrm{c} 1.03, \mathrm{CH}_{2} \mathrm{Cl}_{2}\right)$. 
${ }^{1}$ H NMR (500 MHz, Chloroform- $\left.d\right) \delta 9.52(\mathrm{~s}, 1 \mathrm{H}), 7.36-7.24(\mathrm{~m}, 3 \mathrm{H}), 7.14(\mathrm{~d}, J=8.3 \mathrm{~Hz}, 1 \mathrm{H})$, $6.23(\mathrm{dt}, J=16.9,10.3 \mathrm{~Hz}, 1 \mathrm{H}), 6.08(\mathrm{dd}, J=15.2,10.4 \mathrm{~Hz}, 1 \mathrm{H}), 5.41(\mathrm{dt}, J=15.1,7.5 \mathrm{~Hz}, 1 \mathrm{H}), 5.12$ $(\mathrm{d}, J=16.9 \mathrm{~Hz}, 1 \mathrm{H}), 5.01(\mathrm{~d}, J=10.0 \mathrm{~Hz}, 1 \mathrm{H}), 2.68(\mathrm{~m}, 2 \mathrm{H}), 1.45(\mathrm{~s}, 3 \mathrm{H})$.

${ }^{13}$ C NMR (126 MHz, Chloroform-d) $\delta$ 201.3, 141.7, 136.6, 135.2, 135.1, 130.2, 128.4, 127.8, 127.5, $125.6,116.5,54.0,39.5,19.1$.

HRMS (ESI-TOF) m/z: [M+H] ${ }^{+}$Calcd for $\mathrm{C}_{14} \mathrm{H}_{16} \mathrm{ClO} 235.0884$; Found 235.0890.

IR (film, $\mathbf{c m}^{-1}$ ): 3431, 3084, 2970, 2928, 2850, 2809, 2709, 1725, 1594, 1571, 1415, 1260, 1092 , $1005,902,786,697,443$.

(R,E)-2-methyl-2-(m-tolyl)hepta-4,6-dienal (3f). $0.2 \quad \mathrm{mmol}$ aldehyde and $1.0 \mathrm{mmol}$

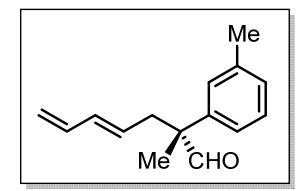
cyclopropylacetylene reacted in the presence of $\mathrm{Pd}\left(\mathrm{PPh}_{3}\right)_{4}(0.005 \mathrm{mmol}), \mathbf{L 6}(0.01$ mmol), A2 (0.12 mmol) and (S)-CPA2 (0.01 mmol) and $3 \AA$ molecular sieves (60 $\mathrm{mg})$ in toluene $(1.0 \mathrm{~mL})$ at $80{ }^{\circ} \mathrm{C}$ for $24 \mathrm{~h}$ following the method A. Flash chromatography $\left(\mathrm{SiO}_{2}\right.$, hexanes/ ethyl ether $\left.=20 / 1\right)$ of crude product gave pure $\mathbf{3 f}$ as a light yellow oil. Yield: $32.1 \mathrm{mg}, 75 \%$; $\mathrm{E} / \mathrm{Z}>20 / 1$; $\mathrm{L} / \mathrm{B}>20 / 1$; ee $=90 \%$, determined by HPLC (AS, hexane/isopropanol $=95 / 5$, flow rate $0.5 \mathrm{~mL} / \mathrm{min}, \mathrm{T}=30{ }^{\circ} \mathrm{C}, 220 \mathrm{~nm}$ ): $\mathrm{t}_{\mathrm{R}}=8.814 \mathrm{~min}$ (major), $\mathrm{t}_{\mathrm{R}}=9.762 \min$ (minor). $[\alpha]_{\mathrm{d}}{ }^{20}=-96.7\left(\mathrm{c} 0.85, \mathrm{CH}_{2} \mathrm{Cl}_{2}\right)$.

${ }^{1}$ H NMR (400 MHz, Chloroform- $d$ ) $\delta 9.50(\mathrm{~s}, 1 \mathrm{H}), 7.29(\mathrm{~d}, J=7.5 \mathrm{~Hz}, 1 \mathrm{H}), 7.11(\mathrm{~d}, J=7.4 \mathrm{~Hz}, 1 \mathrm{H})$, $7.08-7.02(\mathrm{~m}, 2 \mathrm{H}), 6.22(\mathrm{dt}, J=16.8,10.2 \mathrm{~Hz}, 1 \mathrm{H}), 6.07(\mathrm{dd}, J=15.0,10.4 \mathrm{~Hz}, 1 \mathrm{H}), 5.43(\mathrm{dt}, J=$ 15.0, 7.5 Hz, 1H), $5.10(\mathrm{~d}, J=17.3 \mathrm{~Hz}, 1 \mathrm{H}), 4.98(\mathrm{~d}, J=10.5 \mathrm{~Hz}, 1 \mathrm{H}), 2.74-2.62(\mathrm{~m}, 2 \mathrm{H}), 2.36$ (s, $3 \mathrm{H}), 1.42(\mathrm{~s}, 3 \mathrm{H})$.

${ }^{13}$ C NMR (101 MHz, Chloroform-d) $\delta$ 202.1, 139.4, 138.7, 136.8, 134.7, 129.3, 128.9, 128.3, 128.0, $124.3,116.1,54.0,39.5,21.7,19.1$.

HRMS (ESI-TOF) m/z: [M+H] $]^{+}$Calcd for $\mathrm{C}_{15} \mathrm{H}_{19} \mathrm{O}$ 215.1430; Found 215.1435.

IR (film, $\mathbf{c m}^{-1}$ ): 3433, 3084, 2966, 2917, 2849, 2804, 2706, 1724, 1603, 1456, 1260, 1005, 899, 785, $704,442$.

(R,E)-2-(2-methoxyphenyl)-2-methylhepta-4,6-dienal (3g). $0.2 \mathrm{mmol}$ aldehyde and $1.0 \mathrm{mmol}$

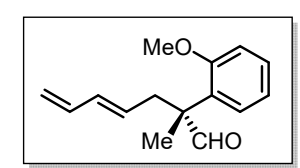
cyclopropylacetylene reacted in the presence of $\mathrm{Pd}\left(\mathrm{PPh}_{3}\right)_{4}(0.01 \mathrm{mmol}), \mathbf{L 6}(0.02$ $\mathrm{mmol}), \mathbf{A 2}(0.12 \mathrm{mmol})$ and (S)-CPA2 $(0.02 \mathrm{mmol})$ and $3 \AA$ molecular sieves $(60$ $\mathrm{mg})$ in toluene $(1.0 \mathrm{~mL})$ at $100{ }^{\circ} \mathrm{C}$ for $48 \mathrm{~h}$ following the method B. Flash chromatography $\left(\mathrm{SiO}_{2}\right.$, hexanes/ ethyl ether $\left.=15 / 1\right)$ of crude product gave pure $\mathbf{3 g}$ as a light yellow oil. Yield: $21.6 \mathrm{mg}, 47 \%$; $\mathrm{E} / \mathrm{Z}>20 / 1$; $/ \mathrm{B}>20 / 1$; ee $=91 \%$, determined by HPLC (AS, hexane/isopropanol $=95 / 5$, flow rate $0.5 \mathrm{~mL} / \mathrm{min}, \mathrm{T}=30{ }^{\circ} \mathrm{C}, 220 \mathrm{~nm}$ ): $\mathrm{t}_{\mathrm{R}}=9.225 \mathrm{~min}$ (major), $\mathrm{t}_{\mathrm{R}}=10.279 \min$ (minor). $[\alpha]_{\mathrm{d}}{ }^{20}=-164.5\left(\mathrm{c} 1.00, \mathrm{CH}_{2} \mathrm{Cl}_{2}\right)$.

${ }^{1}$ H NMR (500 MHz, Chloroform-d) $\delta 9.54(\mathrm{~s}, 1 \mathrm{H}), 7.31(\mathrm{td}, J=7.8,1.6 \mathrm{~Hz}, 1 \mathrm{H}), 7.22(\mathrm{dd}, J=7.7$, $1.7 \mathrm{~Hz}, 1 \mathrm{H}), 7.01(\mathrm{td}, J=7.6,1.2 \mathrm{~Hz}, 1 \mathrm{H}), 6.91(\mathrm{dd}, J=8.1,1.1 \mathrm{~Hz}, 1 \mathrm{H}), 6.20$ (dt, $J=17.0,10.3 \mathrm{~Hz}$, $1 \mathrm{H}), 5.99(\mathrm{dd}, J=15.0,10.5 \mathrm{~Hz}, 1 \mathrm{H}), 5.38(\mathrm{dd}, J=15.2,7.5 \mathrm{~Hz}, 1 \mathrm{H}), 5.06(\mathrm{~d}, J=17.0 \mathrm{~Hz}, 1 \mathrm{H}), 4.94$ $(\mathrm{d}, J=10.1 \mathrm{~Hz}, 1 \mathrm{H}), 3.77(\mathrm{~s}, 3 \mathrm{H}), 2.75(\mathrm{dd}, J=14.1,6.7 \mathrm{~Hz}, 1 \mathrm{H}), 2.64(\mathrm{dd}, J=14.1,8.5 \mathrm{~Hz}, 1 \mathrm{H})$, $1.32(\mathrm{~s}, 3 \mathrm{H})$.

${ }^{13}$ C NMR (126 MHz, Chloroform-d) $\delta$ 203.7, 156.7, 137.0, 134.2, 130.0, 129.8, 128.9, 128.1, 121.1, 115.6, 111.2, 55.4, 51.8, 37.6, 19.8 .

HRMS (ESI-TOF) m/z: [M+H] $]^{+}$Calcd for $\mathrm{C}_{15} \mathrm{H}_{19} \mathrm{O}_{2} 231.1380$; Found 231.1379.

IR (film, $\mathbf{c m}^{-1}$ ): 3436, 3085, 2966, 2933, 2837, 2706, 1727, 1489, 1462, 1242, 1026, 1006, 901, 790, $754,493$. 
(R,E)-2-(2-fluorophenyl)-2-methylhepta-4,6-dienal (3h). $0.2 \mathrm{mmol}$ aldehyde and $1.0 \mathrm{mmol}$

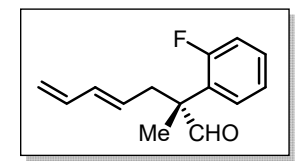
cyclopropylacetylene reacted in the presence of $\mathrm{Pd}\left(\mathrm{PPh}_{3}\right)_{4}(0.005 \mathrm{mmol}), \mathbf{L 6}(0.01$ $\mathrm{mmol}), \mathbf{A 2}(0.12 \mathrm{mmol})$ and (S)-CPA2 $(0.01 \mathrm{mmol})$ and $3 \AA$ molecular sieves $(60$ $\mathrm{mg})$ in toluene $(1.0 \mathrm{~mL})$ at $80{ }^{\circ} \mathrm{C}$ for $24 \mathrm{~h}$ following the method A. Flash chromatography $\left(\mathrm{SiO}_{2}\right.$, hexanes/ ethyl ether $\left.=20 / 1\right)$ of crude product gave pure $\mathbf{3 h}$ as a light yellow oil. Yield: $24.4 \mathrm{mg}, 56 \%$; $/ \mathrm{Z}>20 / 1 ; \mathrm{L} / \mathrm{B}>20 / 1$; ee $=93 \%$, determined by HPLC (OD, hexane/isopropanol $=99.7 / 0.3$, flow rate $0.5 \mathrm{~mL} / \mathrm{min}, \mathrm{T}=30^{\circ} \mathrm{C}, 220 \mathrm{~nm}$ ): $\mathrm{t}_{\mathrm{R}}=14.757$ min (major), $\mathrm{t}_{\mathrm{R}}=15.481 \mathrm{~min}$ (minor). $[\alpha]_{\mathrm{d}}{ }^{20}=-104.5\left(\mathrm{c} 1.10, \mathrm{CH}_{2} \mathrm{Cl}_{2}\right)$.

${ }^{1}$ H NMR (400 MHz, Chloroform- $d$ ) $\delta 9.68(\mathrm{~d}, J=5.0 \mathrm{~Hz}, 1 \mathrm{H}), 7.37-7.26(\mathrm{~m}, 1 \mathrm{H}), 7.30-7.21(\mathrm{~m}$, $3 \mathrm{H}), 7.18(\mathrm{ddd}, J=7.8,7.2,1.3 \mathrm{~Hz}, 1 \mathrm{H}), 7.08(\mathrm{ddd}, J=11.5,8.1,1.2 \mathrm{~Hz}, 1 \mathrm{H}), 6.20$ (dt, $J=16.9,10.4$ $\mathrm{Hz}, 1 \mathrm{H}), 6.03(\mathrm{dd}, J=15.1,10.4 \mathrm{~Hz}, 1 \mathrm{H}), 5.40$ (dt, $J=15.2,7.6 \mathrm{~Hz}, 1 \mathrm{H}), 5.08(\mathrm{~d}, J=16.7 \mathrm{~Hz}, 1 \mathrm{H})$, $4.97(\mathrm{~d}, J=9.8 \mathrm{~Hz}, 1 \mathrm{H}), 2.77(\mathrm{dd}, J=14.2,7.0 \mathrm{~Hz}, 1 \mathrm{H}), 2.66(\mathrm{dd}, J=14.3,8.1 \mathrm{~Hz}, 1 \mathrm{H}), 1.41(\mathrm{~s}, 3 \mathrm{H})$. ${ }^{13}$ C NMR (126 MHz, Chloroform-d) $\delta 202.0\left(\mathrm{C}-\mathrm{F},{ }^{4} \mathrm{~J}_{\mathrm{C}-\mathrm{F}}=2.8 \mathrm{~Hz}\right), 202.0\left(\mathrm{C}-\mathrm{F},{ }^{4} \mathrm{~J}_{\mathrm{C}-\mathrm{F}}=2.8 \mathrm{~Hz}\right), 162.0$ $\left(\mathrm{C}-\mathrm{F},{ }^{1} \mathrm{~J}_{\mathrm{C}-\mathrm{F}}=247.3 \mathrm{~Hz}\right), 160.0\left(\mathrm{C}-\mathrm{F},{ }^{1} \mathrm{~J}_{\mathrm{C}-\mathrm{F}}=247.3 \mathrm{~Hz}\right), 136.7,135.0,129.6\left(\mathrm{C}-\mathrm{F},{ }^{3} \mathrm{~J}_{\mathrm{C}-\mathrm{F}}=8.7 \mathrm{~Hz}\right), 129.6$ $\left(\mathrm{C}-\mathrm{F},{ }^{3} \mathrm{~J}_{\mathrm{C}-\mathrm{F}}=8.7 \mathrm{~Hz}\right), 128.7\left(\mathrm{C}-\mathrm{F},{ }^{3} \mathrm{~J}_{\mathrm{C}-\mathrm{F}}=5.0 \mathrm{~Hz}\right), 128.7\left(\mathrm{C}-\mathrm{F},{ }^{3} \mathrm{~J}_{\mathrm{C}-\mathrm{F}}=5.0 \mathrm{~Hz}\right), 128.6,128.0\left(\mathrm{C}-\mathrm{F},{ }^{2} \mathrm{~J}_{\mathrm{C}-}\right.$ $\mathrm{F}=12.9 \mathrm{~Hz}), 127.9\left(\mathrm{C}-\mathrm{F},{ }^{2} \mathrm{~J}_{\mathrm{C}-\mathrm{F}}=12.9 \mathrm{~Hz}\right), 124.6\left(\mathrm{C}-\mathrm{F},{ }^{4} \mathrm{~J}_{\mathrm{C}-\mathrm{F}}=3.2 \mathrm{~Hz}\right), 124.6\left(\mathrm{C}-\mathrm{F},{ }^{4} \mathrm{~J}_{\mathrm{C}-\mathrm{F}}=3.2 \mathrm{~Hz}\right)$, $116.4\left(\mathrm{C}-\mathrm{F},{ }^{2} \mathrm{~J}_{\mathrm{C}-\mathrm{F}}=22.5 \mathrm{~Hz}\right), 116.3,116.2\left(\mathrm{C}-\mathrm{F},{ }^{2} \mathrm{~J}_{\mathrm{C}-\mathrm{F}}=22.5 \mathrm{~Hz}\right), 52.3\left(\mathrm{C}-\mathrm{F},{ }^{3} \mathrm{~J}_{\mathrm{C}-\mathrm{F}}=3.7 \mathrm{~Hz}\right), 52.3(\mathrm{C}-$ $\left.\mathrm{F},{ }^{3} \mathrm{~J}_{\mathrm{C}-\mathrm{F}}=3.7 \mathrm{~Hz}\right), 38.2\left(\mathrm{C}-\mathrm{F},{ }^{4} \mathrm{~J}_{\mathrm{C}-\mathrm{F}}=2.8 \mathrm{~Hz}\right), 38.2\left(\mathrm{C}-\mathrm{F},{ }^{4} \mathrm{~J}_{\mathrm{C}-\mathrm{F}}=2.8 \mathrm{~Hz}\right), 19.4$.

${ }^{19}$ F NMR (471 MHz, Chloroform-d) $\delta-111.8$.

HRMS (ESI-TOF) m/z: [M+H] $]^{+}$Calcd for $\mathrm{C}_{14} \mathrm{H}_{16} \mathrm{FO} 219.1180$; Found 219.1183.

IR (film, $\mathbf{c m}^{-1}$ ): 3447, 3085, 2972, 2933, 2808, 2710, 1731, 1488, 1448, 1216, 1005, 904, 804, 758, 485.

(R,E)-2-(9H-fluoren-2-yl)-2-methylhepta-4,6-dienal (3i). $0.2 \mathrm{mmol}$ aldehyde and $1.0 \mathrm{mmol}$

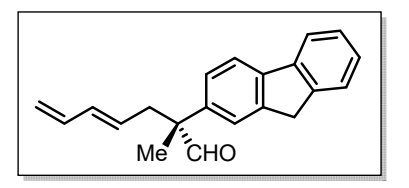
cyclopropylacetylene reacted in the presence of $\mathrm{Pd}\left(\mathrm{PPh}_{3}\right) 4(0.005 \mathrm{mmol}), \mathbf{L 6}$ (0.01 mmol), A2 $(0.12 \mathrm{mmol})$ and (S)-CPA2 $(0.01 \mathrm{mmol})$ and $3 \AA$ molecular sieves $(60 \mathrm{mg})$ in toluene $(1.0 \mathrm{~mL})$ at $80^{\circ} \mathrm{C}$ for $24 \mathrm{~h}$ following the method $\mathrm{A}$. Flash chromatography $\left(\mathrm{SiO}_{2}\right.$, hexanes/ ethyl ether $\left.=20 / 1\right)$ of crude product gave pure 3i as a light yellow oil. Yield: $40.3 \mathrm{mg}, 70 \%$; E/Z $>20 / 1 ; \mathrm{L} / \mathrm{B}>20 / 1$; ee $=90 \%$, determined by HPLC (AS, hexane/isopropanol $=95 / 5$, flow rate $0.5 \mathrm{~mL} / \mathrm{min}, \mathrm{T}=30{ }^{\circ} \mathrm{C}, 254 \mathrm{~nm}$ ): $\mathrm{t}_{\mathrm{R}}=13.283 \mathrm{~min}$ (major), $\mathrm{t}_{\mathrm{R}}=14.986 \mathrm{~min}$ (minor). $[\alpha]_{\mathrm{d}}{ }^{20}=-118.9\left(\mathrm{c} 0.93, \mathrm{CH}_{2} \mathrm{Cl}_{2}\right)$.

${ }^{1}$ H NMR (500 MHz, Chloroform- $\left.d\right) \delta 9.55(\mathrm{~s}, 1 \mathrm{H}), 7.79(\mathrm{~d}, J=8.4 \mathrm{~Hz}, 2 \mathrm{H}), 7.55$ (d, J= $\left.7.4 \mathrm{~Hz}, 1 \mathrm{H}\right)$, $7.44(\mathrm{~s}, 1 \mathrm{H}), 7.39$ (t, $J=7.5 \mathrm{~Hz}, 1 \mathrm{H}), 7.32(\mathrm{t}, J=7.6 \mathrm{~Hz}, 1 \mathrm{H}), 7.27(\mathrm{~d}, J=6.5 \mathrm{~Hz}, 1 \mathrm{H}), 6.22(\mathrm{dt}, J=$ $16.9,10.3 \mathrm{~Hz}, 1 \mathrm{H}), 6.10(\mathrm{dd}, J=15.1,10.4 \mathrm{~Hz}, 1 \mathrm{H}), 5.46(\mathrm{dt}, J=15.0,7.5 \mathrm{~Hz}, 1 \mathrm{H}), 5.10(\mathrm{~d}, J=16.9$ $\mathrm{Hz}, 1 \mathrm{H}), 4.98(\mathrm{~d}, J=10.0 \mathrm{~Hz}, 1 \mathrm{H}), 3.91(\mathrm{~s}, 2 \mathrm{H}), 2.83-2.65(\mathrm{~m}, 2 \mathrm{H}), 1.50$ (s, 3H).

${ }^{13}$ C NMR (126 MHz, Chloroform-d) $\delta$ 202.0, 144.1, 143.5, 141.2, 141.2, 137.8, 136.8, 134.7, 129.3, 127.1, 127.0, 126.0, 125.2, 124.0, 120.3, 120.1, 116.2, 54.2, 39.7, 37.1, 19.2.

HRMS (ESI-TOF) m/z: [M+H] ${ }^{+}$Calcd for $\mathrm{C}_{21} \mathrm{H}_{21} \mathrm{O}$ 289.1587; Found 289.1593.

IR (film, $\mathbf{c m}^{-1}$ ): 3428, 3011, 2968, 2919, 2849, 1722, 1456, 1418, 1260, 1004, 902, 770, 736.

(R,E)-2-methyl-2-(naphthalen-2-yl)hepta-4,6-dienal (3j). $0.2 \mathrm{mmol}$ aldehyde and $1.0 \mathrm{mmol}$

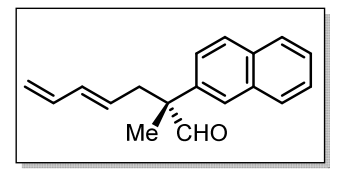
cyclopropylacetylene reacted in the presence of $\mathrm{Pd}\left(\mathrm{PPh}_{3}\right)_{4}(0.005 \mathrm{mmol}), \mathbf{L 6}$ (0.01 mmol), A2 $(0.12 \mathrm{mmol})$ and (S)-CPA2 $(0.01 \mathrm{mmol})$ and $3 \AA$ molecular sieves $(60 \mathrm{mg})$ in toluene $(1.0 \mathrm{~mL})$ at $80{ }^{\circ} \mathrm{C}$ for $24 \mathrm{~h}$ following the method A. 
Flash chromatography $\left(\mathrm{SiO}_{2}\right.$, hexanes/ ethyl ether $\left.=20 / 1\right)$ of crude product gave pure $\mathbf{3} \mathbf{j}$ as a light yellow oil. Yield: $40.5 \mathrm{mg}, 81 \%$; E/Z $>20 / 1 ; \mathrm{L} / \mathrm{B}>20 / 1$; ee $=89 \%$, determined by HPLC (AS, hexane/isopropanol $=92.5 / 7.5$, flow rate $0.5 \mathrm{~mL} / \mathrm{min}, \mathrm{T}=30^{\circ} \mathrm{C}, 230 \mathrm{~nm}$ ): $\mathrm{tR}_{\mathrm{R}}=11.205 \mathrm{~min}$ (major), $\mathrm{t}_{\mathrm{R}}=12.236 \min$ (minor). $[\alpha]_{\mathrm{d}}{ }^{20}=-132.3\left(\mathrm{c} 0.60, \mathrm{CH}_{2} \mathrm{Cl}_{2}\right)$.

${ }^{1} \mathbf{H}$ NMR (400 MHz, Chloroform-d) $\delta 9.59(\mathrm{~s}, 1 \mathrm{H}), 7.91-7.82(\mathrm{~m}, 3 \mathrm{H}), 7.72(\mathrm{~d}, J=2.0 \mathrm{~Hz}, 1 \mathrm{H})$, $7.54-7.47(\mathrm{~m}, 2 \mathrm{H}), 7.37(\mathrm{dd}, J=8.6,2.0 \mathrm{~Hz}, 1 \mathrm{H}), 6.28-6.04(\mathrm{~m}, 2 \mathrm{H}), 5.44(\mathrm{dt}, J=14.9,7.5 \mathrm{~Hz}$, $1 \mathrm{H}), 5.10(\mathrm{~d}, J=15.9 \mathrm{~Hz}, 1 \mathrm{H}), 4.97(\mathrm{~d}, J=9.2 \mathrm{~Hz}, 1 \mathrm{H}), 2.90-2.71(\mathrm{~m}, 2 \mathrm{H}), 1.56(\mathrm{~s}, 3 \mathrm{H})$.

${ }^{13}$ C NMR (126 MHz, Chloroform-d) $\delta$ 202.0, 136.8, 136.8, 134.8, 133.5, 132.6, 129.1, 128.8, 128.2, $127.7,126.5,126.5,126.4,125.1,116.2,54.3,39.5,19.2$.

HRMS (ESI-TOF) m/z: [M+H] $]^{+}$Calcd for $\mathrm{C}_{18} \mathrm{H}_{19} \mathrm{O}$ 251.1430; Found 251.1439.

IR (film, $\mathbf{c m}^{-1}$ ): 3060, 3013, 2971, 2934, 2811, 2707, 1723, 1599, 1506, 1275, 1260, 1005, 902, 816, 764, 749, 476 .

(R,E)-2-ethyl-2-phenylhepta-4,6-dienal (3k). $0.2 \mathrm{mmol}$ aldehyde and $1.0 \mathrm{mmol}$

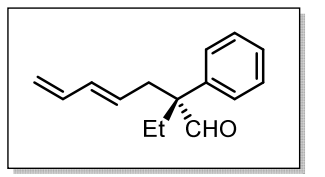
cyclopropylacetylene reacted in the presence of $\mathrm{Pd}\left(\mathrm{PPh}_{3}\right)_{4}(0.01 \mathrm{mmol}), \mathbf{L 6}$ $(0.02 \mathrm{mmol}), \mathbf{A 2}(0.12 \mathrm{mmol})$ and $(\mathrm{S})$-CPA2 $(0.02 \mathrm{mmol})$ and $3 \AA$ molecular sieves $(60 \mathrm{mg})$ in toluene $(1.0 \mathrm{~mL})$ at $100{ }^{\circ} \mathrm{C}$ for $24 \mathrm{~h}$ following the method $\mathrm{B}$. Flash chromatography $\left(\mathrm{SiO}_{2}\right.$, hexanes/ ethyl ether $\left.=20 / 1\right)$ of crude product gave pure 3k as a light yellow oil. Yield: $26.1 \mathrm{mg}, 61 \%$; E/Z $>20 / 1 ; \mathrm{L} / \mathrm{B}>20 / 1$; ee $=84 \%$, determined by HPLC (AS, hexane/isopropanol $=95 / 5$, flow rate $0.5 \mathrm{~mL} / \mathrm{min}, \mathrm{T}=30{ }^{\circ} \mathrm{C}, 220 \mathrm{~nm}$ ): $\mathrm{t}_{\mathrm{R}}=9.448 \mathrm{~min}$ (major), $\mathrm{t}_{\mathrm{R}}=9.977 \mathrm{~min}$ (minor). $[\alpha]_{\mathrm{d}}{ }^{20}=-50.0\left(\mathrm{c} 1.25, \mathrm{CH}_{2} \mathrm{Cl}_{2}\right.$ ).

${ }^{1}$ H NMR (500 MHz, Chloroform-d) $\delta 9.51(\mathrm{~s}, 1 \mathrm{H}), 7.42$ - $7.35(\mathrm{~m}, 2 \mathrm{H}), 7.34-7.28(\mathrm{~m}, 1 \mathrm{H}), 7.25$ $7.16(\mathrm{~m}, 2 \mathrm{H}), 6.21(\mathrm{dt}, J=16.9,10.2 \mathrm{~Hz}, 1 \mathrm{H}), 6.07(\mathrm{dd}, J=15.2,10.4 \mathrm{~Hz}, 1 \mathrm{H}), 5.39(\mathrm{dt}, J=15.0$, $7.5 \mathrm{~Hz}, 1 \mathrm{H}), 5.08$ (dd, $J=16.9,1.7 \mathrm{~Hz}, 1 \mathrm{H}), 4.97(\mathrm{dd}, J=10.2,1.7 \mathrm{~Hz}, 1 \mathrm{H}), 2.77$ (dd, $J=14.6,7.9$ $\mathrm{Hz}, 1 \mathrm{H}), 2.69(\mathrm{dd}, J=14.6,7.1 \mathrm{~Hz}, 1 \mathrm{H}), 1.98(\mathrm{qd}, J=7.3,3.6 \mathrm{~Hz}, 2 \mathrm{H}), 0.80$ (t, $J=7.5 \mathrm{~Hz}, 3 \mathrm{H})$.

${ }^{13}$ C NMR (126 MHz, Chloroform-d) $\delta$ 202.7, 138.6, 136.8, 134.5, 129.0, 128.9, 127.8, 127.5, 116.1, 57.9, 35.2, 24.8, 8.2.

HRMS (ESI-TOF) m/z: [M+H] $]^{+}$Calcd for $\mathrm{C}_{15} \mathrm{H}_{19} \mathrm{O}$ 215.1430; Found 215.1431.

IR (film, $\mathbf{c m}^{-1}$ ): 3085, 3011, 2969, 2922, 2879, 2700, 1724, 1600, 1495,1446, 1004, 902, 759, 700.

\section{(S,E)-2-cyclopropyl-2-phenylhepta-4,6-dienal (3I)}

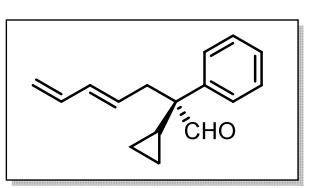

$0.2 \mathrm{mmol}$ aldehyde and $1.0 \mathrm{mmol}$ cyclopropylacetylene reacted in the presence of $\mathrm{Pd}\left(\mathrm{PPh}_{3}\right)_{4}(0.01 \mathrm{mmol}), \mathbf{L 6}(0.02 \mathrm{mmol}), \mathbf{A 2}(0.12 \mathrm{mmol})$ and $(\mathrm{S})-\mathbf{C P A} 2(0.02$ $\mathrm{mmol})$ and $3 \AA$ molecular sieves $(60 \mathrm{mg})$ in toluene $(1.0 \mathrm{~mL})$ at $100{ }^{\circ} \mathrm{C}$ for $24 \mathrm{~h}$ following the method $\mathrm{B}$. Flash chromatography $\left(\mathrm{SiO}_{2}\right.$, hexanes/ ethyl ether $=$ 20/1) of crude product gave pure 31 as a light yellow oil. Yield: $22.6 \mathrm{mg}, 50 \%$; E/Z > 20/1; L/B > 20/1; ee $=84 \%$, determined by HPLC (AS, hexane/isopropanol $=98 / 2$, flow rate $0.4 \mathrm{~mL} / \mathrm{min}, \mathrm{T}=$ $30{ }^{\circ} \mathrm{C}, 220 \mathrm{~nm}$ ): $\mathrm{t}_{\mathrm{R}}=13.688 \mathrm{~min}$ (major), $\mathrm{t}_{\mathrm{R}}=12.949 \mathrm{~min}$ (minor). $[\alpha]_{\mathrm{d}}{ }^{20}=-73.2$ (c $1.20, \mathrm{CH}_{2} \mathrm{Cl}_{2}$ ).

${ }^{1} \mathbf{H}$ NMR (500 MHz, Chloroform-d) $\delta 9.49(\mathrm{~s}, 1 \mathrm{H}), 7.39(\mathrm{t}, J=7.6 \mathrm{~Hz}, 2 \mathrm{H}), 7.34-7.27$ (m, 3H), $6.19(\mathrm{dt}, J=16.9,10.2 \mathrm{~Hz}, 1 \mathrm{H}), 6.06(\mathrm{dd}, J=15.1,10.4 \mathrm{~Hz}, 1 \mathrm{H}), 5.47(\mathrm{dt}, J=15.0,7.5 \mathrm{~Hz}, 1 \mathrm{H})$, $5.07(\mathrm{~d}, J=16.9 \mathrm{~Hz}, 1 \mathrm{H}), 4.95(\mathrm{~d}, J=10.7 \mathrm{~Hz}, 1 \mathrm{H}), 2.75(\mathrm{dd}, J=14.5,6.9 \mathrm{~Hz}, 1 \mathrm{H}), 2.62(\mathrm{dd}, J=$ $14.5,8.1 \mathrm{~Hz}, 1 \mathrm{H}), 1.34(\mathrm{tt}, J=8.6,5.7 \mathrm{~Hz}, 1 \mathrm{H}), 0.68-0.58(\mathrm{~m}, 2 \mathrm{H}), 0.38-0.24(\mathrm{~m}, 2 \mathrm{H})$.

${ }^{13}$ C NMR (126 MHz, Chloroform-d) $\delta 201.9,138.4,136.9,134.5,129.5,128.8,128.5,127.6$, 115.9, 56.7, 37.7, 15.3, 2.0, 0.7.

HRMS (ESI-TOF) m/z: [M+H] $]^{+}$Calcd for $\mathrm{C}_{16} \mathrm{H}_{19} \mathrm{O}$ 227.1430; Found 227.1433. 
IR (film, cm-1): 3083, 3010, 2920, 2848, 2716, 1723, 1600, 1493, 1446, 1004, 900, 761, 701.

(R,E)-1-(penta-2,4-dien-1-yl)-2,3-dihydro-1H-indene-1-carbaldehyde (3m). 0.2 mmol aldehyde

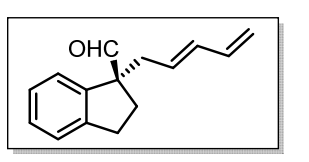
and $1.0 \mathrm{mmol}$ cyclopropylacetylene reacted in the presence of $\mathrm{Pd}\left(\mathrm{PPh}_{3}\right)_{4}(0.005$ mmol), L6 (0.01 mmol), A2 $(0.12 \mathrm{mmol})$ and (S)-CPA2 $(0.01 \mathrm{mmol})$ and $3 \AA$ molecular sieves $(60 \mathrm{mg})$ in toluene $(1.0 \mathrm{~mL})$ at $80{ }^{\circ} \mathrm{C}$ for $24 \mathrm{~h}$ following the method A. Flash chromatography $\left(\mathrm{SiO}_{2}\right.$, hexanes/ ethyl ether $\left.=20 / 1\right)$ of crude product gave pure $\mathbf{3 m}$ as a light yellow oil. Yield: $28.0 \mathrm{mg}, 66 \%$; $\mathrm{E} / \mathrm{Z}>20 / 1 ; \mathrm{L} / \mathrm{B}>20 / 1$; ee $=84 \%$, determined by HPLC (AS, hexane/isopropanol $=95 / 5$, flow rate $0.5 \mathrm{~mL} / \mathrm{min}, \mathrm{T}=30{ }^{\circ} \mathrm{C}, 220 \mathrm{~nm}$ ): $\mathrm{t}_{\mathrm{R}}=10.877 \mathrm{~min}$ (major), $\mathrm{t}_{\mathrm{R}}=14.554 \min$ (minor). $[\alpha]_{\mathrm{d}}{ }^{20}=-88.6\left(\mathrm{c} 0.93, \mathrm{CH}_{2} \mathrm{Cl}_{2}\right)$.

${ }^{1}$ H NMR (400 MHz, Chloroform-d) $\delta 9.55(\mathrm{~s}, 1 \mathrm{H}), 7.31-7.22(\mathrm{~m}, 3 \mathrm{H}), 7.19-7.14(\mathrm{~m}, 1 \mathrm{H}), 6.23$ (dt, $J=16.8,10.2 \mathrm{~Hz}, 1 \mathrm{H}), 6.09$ (dd, $J=15.1,10.4 \mathrm{~Hz}, 1 \mathrm{H}), 5.49$ (dt, $J=15.0,7.5 \mathrm{~Hz}, 1 \mathrm{H}), 5.10$ (d, $J=16.9 \mathrm{~Hz}, 1 \mathrm{H}), 4.99(\mathrm{~d}, J=10.0 \mathrm{~Hz}, 1 \mathrm{H}), 2.98(\mathrm{t}, J=7.4 \mathrm{~Hz}, 2 \mathrm{H}), 2.75(\mathrm{dd}, J=14.2,8.0 \mathrm{~Hz}, 1 \mathrm{H})$, $2.59-2.47(\mathrm{~m}, 2 \mathrm{H}), 2.06(\mathrm{dt}, J=13.3,7.8 \mathrm{~Hz}, 1 \mathrm{H})$.

${ }^{13}$ C NMR (126 MHz, Chloroform-d) $\delta 200.9,145.1,141.5,136.8,134.6,129.3,128.4,127.0,125.3$, 124.3, 116.2, 63.7, 37.7, 31. 0, 30.6.

HRMS (ESI-TOF) m/z: [M+H] $]^{+}$Calcd for $\mathrm{C}_{15} \mathrm{H}_{17} \mathrm{O}$ 213.1274; Found 213.1276.

IR (film, $\mathbf{~ c m}^{-1}$ ): 3425, 3012, 2927, 2852, 2712, 1723, 1477, 1456, 1260, 1095, 1005, 902, 800, 757.

(R,E)-1-(penta-2,4-dien-1-yl)-1,2,3,4-tetrahydronaphthalene-1-carbaldehyde (3n). 0.2 mmol

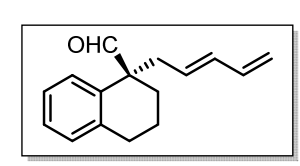
aldehyde and $1.0 \mathrm{mmol}$ cyclopropylacetylene reacted in the presence of $\mathrm{Pd}\left(\mathrm{PPh}_{3}\right)_{4}$ (0.005 $\mathrm{mmol}), \mathbf{L 6}(0.01 \mathrm{mmol}), \mathbf{A 2}(0.12 \mathrm{mmol})$ and $(\mathrm{S})-\mathbf{C P A 2}(0.01 \mathrm{mmol})$ and $3 \AA$ molecular sieves $(60 \mathrm{mg})$ in toluene $(1.0 \mathrm{~mL})$ at $80{ }^{\circ} \mathrm{C}$ for $24 \mathrm{~h}$ following the method A. Flash chromatography $\left(\mathrm{SiO}_{2}\right.$, hexanes/ ethyl ether $\left.=20 / 1\right)$ of crude product gave pure 3n as a light yellow oil. Yield: $27.1 \mathrm{mg}, 60 \%$; E/Z $>20 / 1$; L/B $>20 / 1$; ee $=88 \%$, determined by HPLC (AS, hexane/isopropanol $=95 / 5$, flow rate $0.5 \mathrm{~mL} / \mathrm{min}, \mathrm{T}=30{ }^{\circ} \mathrm{C}, 220 \mathrm{~nm}$ ): $\mathrm{t}_{\mathrm{R}}=9.708$ min (major), $\mathrm{t}_{\mathrm{R}}=15.472 \mathrm{~min}$ (minor). $[\alpha]_{\mathrm{d}}{ }^{20}=-68.8\left(\mathrm{c} 0.50, \mathrm{CH}_{2} \mathrm{Cl}_{2}\right)$.

${ }^{1}$ H NMR (500 MHz, Chloroform-d) $\delta 9.54(\mathrm{~s}, 1 \mathrm{H}), 7.24-7.14(\mathrm{~m}, 4 \mathrm{H}), 6.24(\mathrm{dt}, J=16.9,10.3 \mathrm{~Hz}$, $1 \mathrm{H}), 6.07(\mathrm{dd}, J=15.1,10.5 \mathrm{~Hz}, 1 \mathrm{H}), 5.48(\mathrm{dt}, J=15.1,7.5 \mathrm{~Hz}, 1 \mathrm{H}), 5.10$ (d, $J=17.0 \mathrm{~Hz}, 1 \mathrm{H}), 4.98$ $(\mathrm{d}, J=10.0 \mathrm{~Hz}, 1 \mathrm{H}), 2.81-2.76(\mathrm{~m}, 2 \mathrm{H}), 2.65(\mathrm{~d}, J=7.5 \mathrm{~Hz}, 2 \mathrm{H}), 2.12-2.04(\mathrm{~m}, 1 \mathrm{H}), 1.87-1.76$ $(\mathrm{m}, 3 \mathrm{H})$.

${ }^{13}$ C NMR (126 MHz, Chloroform-d) $\delta$ 202.2, 138.8, 136.9, 134.6, 134.0, 130.1, 129.8, 128.4, 127.2, $126.5,116.1,53.5,40.1,30.0,28.1,19.3$.

HRMS (ESI-TOF) m/z: [M+H] $]^{+}$Calcd for $\mathrm{C}_{16} \mathrm{H}_{19} \mathrm{O} 227.1430$; Found 227.1432.

IR (film, $\mathbf{c m}^{-1}$ ): 3018, 2934, 2872, 2710, 1722, 1600, 1445, 1263, 1005, 899, 799, 750.

(R,E)-5-(penta-2,4-dien-1-yl)-6,7,8,9-tetrahydro-5H-benzo[7]annulene-5-carbaldehyde

(30).

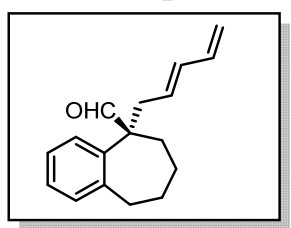

$0.2 \mathrm{mmol}$ aldehyde and $1.0 \mathrm{mmol}$ cyclopropylacetylene reacted in the presence of $\mathrm{Pd}\left(\mathrm{PPh}_{3}\right)_{4}(0.005 \mathrm{mmol}), \mathbf{L 6}(0.01 \mathrm{mmol}), \mathbf{A 2}(0.12 \mathrm{mmol})$ and (S)-CPA2 $(0.01$ $\mathrm{mmol})$ and $3 \AA$ molecular sieves $(60 \mathrm{mg})$ in toluene $(1.0 \mathrm{~mL})$ at $80{ }^{\circ} \mathrm{C}$ for $24 \mathrm{~h}$ following the method A. Flash chromatography $\left(\mathrm{SiO}_{2}\right.$, hexanes/ ethyl ether $\left.=20 / 1\right)$ of crude product gave pure 30 as a light yellow oil. Yield: $30.7 \mathrm{mg}, 64 \%$; E/Z > 20/1; L/B > 20/1; ee $=94 \%$, determined by HPLC (AS, hexane/isopropanol $=95 / 5$, flow rate 0.5 $\left.\mathrm{mL} / \mathrm{min}, \mathrm{T}=30^{\circ} \mathrm{C}, 220 \mathrm{~nm}\right): \quad \mathrm{t}_{\mathrm{R}}=9.119 \min$ (major), $\mathrm{t}_{\mathrm{R}}=10.257 \mathrm{~min}$ (minor). $[\alpha]_{\mathrm{d}}{ }^{20}=6.6(\mathrm{c} 0.80$, $\mathrm{CH}_{2} \mathrm{Cl}_{2}$ ). 
${ }^{1}$ H NMR (500 MHz, Chloroform-d) $\delta 9.61(\mathrm{~s}, 1 \mathrm{H}), 7.23-7.12(\mathrm{~m}, 3 \mathrm{H}), 7.07-7.01(\mathrm{~m}, 1 \mathrm{H}), 6.25$ $(\mathrm{dt}, J=16.9,10.3 \mathrm{~Hz}, 1 \mathrm{H}), 6.11(\mathrm{dd}, J=15.2,10.4 \mathrm{~Hz}, 1 \mathrm{H}), 5.58(\mathrm{dt}, J=14.9,7.4 \mathrm{~Hz}, 1 \mathrm{H}), 5.10$ (d, $J=16.9 \mathrm{~Hz}, 1 \mathrm{H}), 4.99(\mathrm{~d}, J=10.0 \mathrm{~Hz}, 1 \mathrm{H}), 2.91(\mathrm{ddd}, J=13.7,9.0,4.2 \mathrm{~Hz}, 1 \mathrm{H}), 2.74-2.65$ (m, $3 \mathrm{H}), 2.08-1.99(\mathrm{~m}, 1 \mathrm{H}), 1.84-1.66(\mathrm{~m}, 5 \mathrm{H})$.

${ }^{13}$ C NMR (126 MHz, Chloroform-d) $\delta$ 202.8, 142.4, 138.6, 136.9, 134.8, 131.4, 129.7, 128.7, 127.6, 126.6, 116.1, 58.2, 39.2, 34.4, 30.7, 26.6, 23.5.

HRMS (ESI-TOF) m/z: [M+H] $]^{+}$Calcd for $\mathrm{C}_{17} \mathrm{H}_{21} \mathrm{O}$ 241.1587; Found 241.1586.

IR (film, $\mathbf{c m}^{-1}$ ): 3015, 2928, 2856, 2710, 1723, 1489, 1447, 1260, 1095, 1005, 899, 800, 756.

(R,E)-2-methyl-2-(pyridin-2-yl)hepta-4,6-dienal (3p). $0.2 \mathrm{mmol}$ aldehyde and $1.0 \mathrm{mmol}$

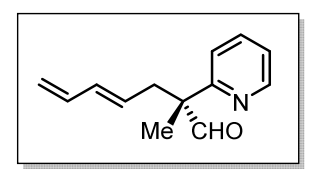

cyclopropylacetylene reacted in the presence of $\mathrm{Pd}\left(\mathrm{PPh}_{3}\right)_{4}(0.005 \mathrm{mmol}), \mathbf{L 6}$ (0.01 mmol), A2 $(0.12 \mathrm{mmol})$ and (S)-CPA2 $(0.01 \mathrm{mmol})$ and $3 \AA$ molecular sieves $(60 \mathrm{mg})$ in toluene $(1.0 \mathrm{~mL})$ at $80{ }^{\circ} \mathrm{C}$ for $24 \mathrm{~h}$ following the method A. Flash chromatography $\left(\mathrm{SiO}_{2}\right.$, hexanes/ ethyl ether $\left.=12 / 1\right)$ of crude product gave pure 3p as a light yellow oil. Yield: $24.1 \mathrm{mg}, 60 \%$; $\mathrm{E} / \mathrm{Z}>20 / 1 ; \mathrm{L} / \mathrm{B}>20 / 1$; ee $=81 \%$, determined by HPLC (ID, hexane/isopropanol $=95 / 5$, flow rate $1.0 \mathrm{~mL} / \mathrm{min}, \mathrm{T}=30^{\circ} \mathrm{C}, 220 \mathrm{~nm}$ ): $\mathrm{t}_{\mathrm{R}}=6.823 \mathrm{~min}$ (major), $\mathrm{t}_{\mathrm{R}}=6.182 \mathrm{~min}$ (minor). $[\alpha]_{\mathrm{d}}{ }^{20}=-28.2\left(\mathrm{c} 0.91, \mathrm{CH}_{2} \mathrm{Cl}_{2}\right)$.

${ }^{1}$ H NMR (400 MHz, Chloroform-d) $\delta 9.79(\mathrm{~s}, 1 \mathrm{H}), 8.61$ (ddd, J = 4.8, 1.9, $\left.1.0 \mathrm{~Hz}, 1 \mathrm{H}\right), 7.69$ (td, J = 7.8, $1.9 \mathrm{~Hz}, 1 \mathrm{H}), 7.25-7.15(\mathrm{~m}, 2 \mathrm{H}), 6.21(\mathrm{dt}, \mathrm{J}=16.9,10.3 \mathrm{~Hz}, 1 \mathrm{H}), 6.03$ (dd, J = 15.1, $10.4 \mathrm{~Hz}$,

1H), $5.45(\mathrm{dt}, \mathrm{J}=15.1,7.6 \mathrm{~Hz}, 1 \mathrm{H}), 5.07(\mathrm{~d}, \mathrm{~J}=17.1 \mathrm{~Hz}, 1 \mathrm{H}), 4.96(\mathrm{~d}, \mathrm{~J}=10.3 \mathrm{~Hz}, 1 \mathrm{H}), 2.77(\mathrm{~m}$ $2 \mathrm{H}), 1.45$ (s, 3H).

${ }^{13}$ C NMR (101 MHz, Chloroform-d) $\delta$ 203.0, 160.9, 149.5, 136.9, 136.8, 134.8, 128.9, 122.1, $121.6,116.2,56.3,39.5,18.8$.

HRMS (ESI-TOF) m/z: [M+H] $]^{+}$Calcd for $\mathrm{C}_{13} \mathrm{H}_{16} \mathrm{NO} 202.1226$; Found 202.1230.

IR (film, $\mathbf{c m}^{-1}$ ): 3084, 2973, 2924, 2851, 1725, 1588, 1570, 1470, 1432, 1004, 993, 786, 750.

(S,E)-2-methyl-2-(thiophen-2-yl)hepta-4,6-dienal (3q). $0.2 \mathrm{mmol}$ aldehyde and $1.0 \mathrm{mmol}$

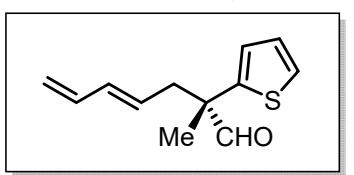
cyclopropylacetylene reacted in the presence of $\mathrm{Pd}\left(\mathrm{PPh}_{3}\right)_{4}(0.005 \mathrm{mmol}), \mathbf{L 6}$ $(0.01 \mathrm{mmol}), \mathbf{A 2}(0.12 \mathrm{mmol})$ and $(\mathrm{S})-\mathbf{C P A} 2(0.01 \mathrm{mmol})$ and $3 \AA$ molecular sieves $(60 \mathrm{mg})$ in toluene $(1.0 \mathrm{~mL})$ at $80{ }^{\circ} \mathrm{C}$ for $24 \mathrm{~h}$ following the method $\mathrm{A}$. Flash chromatography $\left(\mathrm{SiO}_{2}\right.$, hexanes/ ethyl ether $\left.=15 / 1\right)$ of crude product gave pure 3q as a light yellow oil. Yield: $21.4 \mathrm{mg}, 52 \%$; E/Z $>20 / 1 ; \mathrm{L} / \mathrm{B}>20 / 1$; ee $=86 \%$, determined by HPLC (AS, hexane/isopropanol $=95 / 5$, flow rate $0.5 \mathrm{~mL} / \mathrm{min}, \mathrm{T}=30^{\circ} \mathrm{C}, 220 \mathrm{~nm}$ ): $\mathrm{t}_{\mathrm{R}}=9.783$ $\min$ (major), $\mathrm{t}_{\mathrm{R}}=10.621 \mathrm{~min}$ (minor). $[\alpha]_{\mathrm{d}}{ }^{20}=-34.1\left(\mathrm{c} 0.80, \mathrm{CH}_{2} \mathrm{Cl}_{2}\right)$.

${ }^{1}$ H NMR (400 MHz, Chloroform-d) $\delta 9.48(\mathrm{~s}, 1 \mathrm{H}), 7.31(\mathrm{dd}, \mathrm{J}=5.1,1.2 \mathrm{~Hz}, 1 \mathrm{H}), 7.04$ (dd, J = 5.1, $3.6 \mathrm{~Hz}, 1 \mathrm{H}), 6.91(\mathrm{dd}, \mathrm{J}=3.6,1.2 \mathrm{~Hz}, 1 \mathrm{H}), 6.25(\mathrm{dt}, \mathrm{J}=16.9,10.3 \mathrm{~Hz}, 1 \mathrm{H}), 6.11(\mathrm{dd}, \mathrm{J}=15.0,10.5$ $\mathrm{Hz}, 1 \mathrm{H}), 5.52(\mathrm{dt}, \mathrm{J}=15.0,7.5 \mathrm{~Hz}, 1 \mathrm{H}), 5.12(\mathrm{~d}, \mathrm{~J}=16.3 \mathrm{~Hz}, 1 \mathrm{H}), 5.01(\mathrm{~d}, \mathrm{~J}=10.2 \mathrm{~Hz}, 1 \mathrm{H}), 2.71$ $(\mathrm{d}, \mathrm{J}=7.5 \mathrm{~Hz}, 2 \mathrm{H}), 1.50(\mathrm{~s}, 3 \mathrm{H})$.

${ }^{13}$ C NMR (101 MHz, Chloroform-d) $\delta$ 199.7, 144.2, 136.7, 135.3, 128.3, 127.6, 125.6, 125.4, 116.6, 52.6, 40.4, 20.2 .

HRMS (ESI-TOF) m/z: [M+H] $]^{+}$Calcd for $\mathrm{C}_{12} \mathrm{H}_{15} \mathrm{OS} 207.0838$; Found 207.0841.

IR (film, $\mathbf{c m}^{-1}$ ): 2972, 2929, 2806, 2706, 1727, 1455, 1435, 1238, 1004, 904, 701. 
(R,4E,6E)-2-methyl-2,7-diphenylhepta-4,6-dienal (3r). $0.2 \mathrm{mmol}$ aldehyde and $0.4 \mathrm{mmol}$

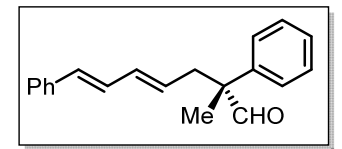
cyclopropylacetylene reacted in the presence of $\mathrm{Pd}\left(\mathrm{PPh}_{3}\right)_{4}(0.01 \mathrm{mmol}), \mathbf{L 6}$ (0.02 mmol), A2 (0.12 mmol) and (S)-CPA2 $(0.02 \mathrm{mmol})$ and $3 \AA$ molecular sieves $(60 \mathrm{mg})$ in toluene $(1.0 \mathrm{~mL})$ at $100{ }^{\circ} \mathrm{C}$ for $48 \mathrm{~h}$ following the method $\mathrm{C}$. Flash chromatography $\left(\mathrm{SiO}_{2}\right.$, hexanes/ ethyl ether $\left.=20 / 1\right)$ of crude product gave pure $3 \mathbf{r}$ as a light yellow oil. Yield: $46.9 \mathrm{mg}, 85 \%$; $\mathrm{E} / \mathrm{Z}>20 / 1$; ee $=89 \%$, determined by HPLC (OD, hexane/isopropanol $=97 / 3$, flow rate $1.0 \mathrm{~mL} / \mathrm{min}, \mathrm{T}=30{ }^{\circ} \mathrm{C}, 254 \mathrm{~nm}$ ): $\mathrm{t}_{\mathrm{R}}=9.560 \mathrm{~min}$ (major), $\mathrm{t}_{\mathrm{R}}=$ 11.100 min (minor). $[\alpha]_{\mathrm{d}^{20}}=-90.8\left(\mathrm{c} 1.20, \mathrm{CH}_{2} \mathrm{Cl}_{2}\right),\left([\alpha]_{\mathrm{d}}{ }^{20}=-96.2\left(\mathrm{c} 0.57, \mathrm{CHCl}_{3}\right)\right)$. The absolute configuration of $\mathbf{3 r}$ was determined by comparison of the optical data with literature reports $((S)-3 \mathbf{r}$ : $\left.[\alpha]_{\mathrm{d}^{20}}=+156.7\left(\mathrm{c} 0.41, \mathrm{CHCl}_{3}\right)\right)^{[2]}$, and the absolute configuration of the other products were tentatively assigned by analogy.

${ }^{1}$ H NMR (500 MHz, Chloroform-d) $\delta 9.47(\mathrm{~s}, 1 \mathrm{H}), 7.35-7.31(\mathrm{~m}, 2 \mathrm{H}), 7.28-7.19(\mathrm{~m}, 7 \mathrm{H}), 7.14-$ $7.10(\mathrm{~m}, 1 \mathrm{H}), 6.59$ (dd, $J=15.7,10.4 \mathrm{~Hz}, 1 \mathrm{H}), 6.36(\mathrm{~d}, J=15.7 \mathrm{~Hz}, 1 \mathrm{H}), 6.15(\mathrm{dd}, J=15.0,10.4 \mathrm{~Hz}$, $1 \mathrm{H}), 5.46(\mathrm{dt}, J=15.1,7.6 \mathrm{~Hz}, 1 \mathrm{H}), 2.73-2.62(\mathrm{~m}, 2 \mathrm{H}), 1.40(\mathrm{~s}, 3 \mathrm{H})$.

${ }^{13}$ C NMR (126 MHz, Chloroform-d) $\delta$ 202.1, 139.5, 137.4, 134.3, 131.3, 129.4, 129.0, 128.8, 128.7, $127.5,127.5,127.3,126.3,54.2,39.9,19.1$.

HRMS (ESI-TOF) m/z: [M+H] $]^{+}$Calcd for $\mathrm{C}_{20} \mathrm{H}_{21} \mathrm{O}$ 277.1587; Found 277.1584.

IR (film, cm-1): 3428, 3028, 2968, 2925, 2708, 1722, 1597, 1494, 1445, 1261, 989, 759, 748, 698.

(R,4E,6E)-2-methyl-2-phenyl-7-(p-tolyl)hepta-4,6-dienal (3s). $0.2 \mathrm{mmol}$ aldehyde and $1.0 \mathrm{mmol}$

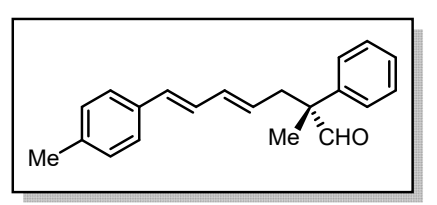
cyclopropylacetylene reacted in the presence of $\mathrm{Pd}\left(\mathrm{PPh}_{3}\right)_{4}(0.01 \mathrm{mmol})$, L6 (0.02 $\mathrm{mmol}), \mathbf{A 2}(0.12 \mathrm{mmol})$ and (S)-CPA2 $(0.02 \mathrm{mmol})$ and $3 \AA$ molecular sieves $(60 \mathrm{mg})$ in toluene $(1.0 \mathrm{~mL})$ at $100{ }^{\circ} \mathrm{C}$ for $48 \mathrm{~h}$ following the method C. Flash chromatography $\left(\mathrm{SiO}_{2}\right.$, hexanes/ ethyl ether $\left.=20 / 1\right)$ of crude product gave pure $3 \mathrm{~s}$ as a light yellow oil. Yield: $54.0 \mathrm{mg}, 93 \%$; E/Z $>20 / 1$; ee $=88 \%$, determined by HPLC (OD, hexane/isopropanol $=95 / 5$, flow rate $0.5 \mathrm{~mL} / \mathrm{min}, \mathrm{T}=30{ }^{\circ} \mathrm{C}, 254 \mathrm{~nm}$ ): $\mathrm{t}_{\mathrm{R}}=13.983 \mathrm{~min}$ (major), $\mathrm{t}_{\mathrm{R}}=15.793 \mathrm{~min}$ (minor). $[\alpha]_{\mathrm{d}}{ }^{20}=-96.8\left(\mathrm{c} 1.15, \mathrm{CH}_{2} \mathrm{Cl}_{2}\right)$.

${ }^{1} \mathbf{H}$ NMR (500 MHz, Chloroform-d) $\delta 9.52(\mathrm{~s}, 1 \mathrm{H}), 7.41-7.35(\mathrm{~m}, 2 \mathrm{H}), 7.32-7.20(\mathrm{~m}, 5 \mathrm{H}), 7.07$ $(\mathrm{d}, J=7.9 \mathrm{~Hz}, 2 \mathrm{H}), 6.60(\mathrm{dd}, J=15.7,10.4 \mathrm{~Hz}, 1 \mathrm{H}), 6.39$ (d, $J=15.7 \mathrm{~Hz}, 1 \mathrm{H}), 6.19$ (dd, $J=15.0$, $10.4 \mathrm{~Hz}, 1 \mathrm{H}), 5.48$ (dt, $J=15.1,7.6 \mathrm{~Hz}, 1 \mathrm{H}), 2.78-2.66(\mathrm{~m}, 2 \mathrm{H}), 2.30(\mathrm{~s}, 3 \mathrm{H}), 1.45(\mathrm{~s}, 3 \mathrm{H})$.

${ }^{13}$ C NMR (126 MHz, Chloroform-d) $\delta$ 202.1, 139.5, 137.3, 134.6, 134.5, 131.3, 129.4, 129.0, 128.7, $127.9,127.5,127.3,126.3,54.2,39.9,21.3,19.1$.

HRMS (ESI-TOF) m/z: [M+H] $]^{+}$Calcd for $\mathrm{C}_{21} \mathrm{H}_{23} \mathrm{O}$ 291.1743; Found 291.1750.

IR (film, $\mathbf{c m}^{-1}$ ): 3022, 2921, 2806, 2708, 1723, 1510, 1445, 988, 815, 760, 699, 511.

(R,4E,6E)-7-(4-fluorophenyl)-2-methyl-2-phenylhepta-4,6-dienal (3t). $0.2 \mathrm{mmol}$ aldehyde and

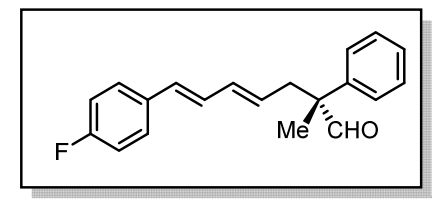
$0.4 \mathrm{mmol}$ cyclopropylacetylene reacted in the presence of $\mathrm{Pd}\left(\mathrm{PPh}_{3}\right)_{4}(0.01$ mmol), L6 (0.02 mmol), A2 (0.12 mmol) and (S)-CPA2 (0.02 mmol) and $3 \AA$ molecular sieves $(60 \mathrm{mg})$ in toluene $(1.0 \mathrm{~mL})$ at $100{ }^{\circ} \mathrm{C}$ for $48 \mathrm{~h}$ following the method $\mathrm{C}$. Flash chromatography $\left(\mathrm{SiO}_{2}\right.$, hexanes/ ethyl ether $=20 / 1$ ) of crude product gave pure 3t as a light yellow oil. Yield: $55.9 \mathrm{mg}, 95 \%$; E/Z > 20/1; ee $=88 \%$, determined by HPLC (OD, hexane/isopropanol $=95 / 5$, flow rate $0.5 \mathrm{~mL} / \mathrm{min}, \mathrm{T}=30{ }^{\circ} \mathrm{C}$,

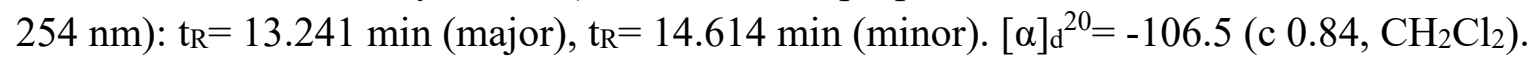


${ }^{1}$ H NMR $(500 \mathrm{MHz}$, Chloroform- $d) \delta 9.52(\mathrm{~s}, 1 \mathrm{H}), 7.38(\mathrm{t}, J=7.6 \mathrm{~Hz}, 2 \mathrm{H}), 7.32-7.23(\mathrm{~m}, 5 \mathrm{H}), 6.95$ (t, $J=8.5 \mathrm{~Hz}, 2 \mathrm{H}), 6.55(\mathrm{dd}, J=15.7,10.4 \mathrm{~Hz}, 1 \mathrm{H}), 6.37(\mathrm{~d}, J=15.7 \mathrm{~Hz}, 1 \mathrm{H}), 6.18$ (dd, $J=15.0$, $10.4 \mathrm{~Hz}, 1 \mathrm{H}), 5.51(\mathrm{dt}, J=15.1,7.5 \mathrm{~Hz}, 1 \mathrm{H}), 2.80-2.66(\mathrm{~m}, 2 \mathrm{H}), 1.45(\mathrm{~s}, 3 \mathrm{H})$.

${ }^{13} \mathrm{C}$ NMR (126 MHz, Chloroform-d) $\delta 202.0,163.2\left(\mathrm{C}-\mathrm{F},{ }^{1} \mathrm{~J}_{\mathrm{C}-\mathrm{F}}=247.3 \mathrm{~Hz}\right), 161.3\left(\mathrm{C}-\mathrm{F},{ }^{1} \mathrm{~J}_{\mathrm{C}-\mathrm{F}}=247.3\right.$ $\mathrm{Hz}), 139.5,134.1,133.6\left(\mathrm{C}-\mathrm{F},{ }^{4} \mathrm{~J}_{\mathrm{C}-\mathrm{F}}=3.2 \mathrm{~Hz}\right), 133.6\left(\mathrm{C}-\mathrm{F},{ }^{4} \mathrm{~J}_{\mathrm{C}-\mathrm{F}}=3.2 \mathrm{~Hz}\right), 130.0,129.4,129.0,128.6$ $\left(\mathrm{C}-\mathrm{F},{ }^{5} \mathrm{~J}_{\mathrm{C}-\mathrm{F}}=2.3 \mathrm{~Hz}\right), 128.6\left(\mathrm{C}-\mathrm{F},{ }^{5} \mathrm{~J}_{\mathrm{C}-\mathrm{F}}=2.3 \mathrm{~Hz}\right), 127.8\left(\mathrm{C}-\mathrm{F},{ }^{3} \mathrm{~J}_{\mathrm{C}-\mathrm{F}}=7.8 \mathrm{~Hz}\right), 127.7\left(\mathrm{C}-\mathrm{F},{ }^{3} \mathrm{~J}_{\mathrm{C}-\mathrm{F}}=7.8\right.$ $\mathrm{Hz}), 127.5,127.3,115.7\left(\mathrm{C}-\mathrm{F},{ }^{2} \mathrm{~J}_{\mathrm{C}-\mathrm{F}}=22.1 \mathrm{~Hz}\right), 115.5\left(\mathrm{C}-\mathrm{F},{ }^{2} \mathrm{~J}_{\mathrm{C}-\mathrm{F}}=22.1 \mathrm{~Hz}\right), 54.2,39.8,19.1$.

${ }^{19}$ F NMR (376 MHz, Chloroform-d) $\delta-114.5$.

HRMS (ESI-TOF) m/z: [M+H] $]^{+}$Calcd for $\mathrm{C}_{20} \mathrm{H}_{20} \mathrm{FO} 295.1493$; Found 295.1502.

IR (film, $\mathbf{c m}^{-1}$ ): 3428, 3023, 2973, 2930, 2807, 2709, 1723, 1599, 1507, 1445, 1228, 1157, 988, 828, $761,700,518$.

(R,4E,6E)-7-(4-chlorophenyl)-2-methyl-2-phenylhepta-4,6-dienal (3u). $0.2 \mathrm{mmol}$ aldehyde and

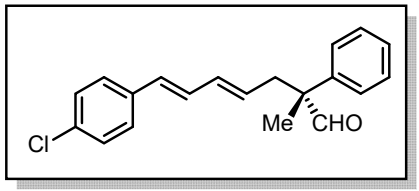
$0.4 \mathrm{mmol}$ cyclopropylacetylene reacted in the presence of $\mathrm{Pd}\left(\mathrm{PPh}_{3}\right)_{4}(0.01$ mmol), L6 (0.02 mmol), A2 (0.12 mmol) and (S)-CPA2 (0.02 mmol) and $3 \AA$ molecular sieves $(60 \mathrm{mg})$ in toluene $(1.0 \mathrm{~mL})$ at $100{ }^{\circ} \mathrm{C}$ for $48 \mathrm{~h}$ following the method $\mathrm{C}$. Flash chromatography $\left(\mathrm{SiO}_{2}\right.$, hexanes/ ethyl ether $=20 / 1$ ) of crude product gave pure $3 \mathbf{u}$ as a light yellow oil. Yield: $57.7 \mathrm{mg}, 93 \%$; E/Z $>20 / 1$; ee $=88 \%$, determined by HPLC (OD, hexane/isopropanol $=95 / 5$, flow rate $0.5 \mathrm{~mL} / \mathrm{min}, \mathrm{T}=30{ }^{\circ} \mathrm{C}$, $220 \mathrm{~nm}$ ): $\mathrm{t}_{\mathrm{R}}=13.886 \min$ (major), $\mathrm{t}_{\mathrm{R}}=14.850 \min$ (minor). $[\alpha]_{\mathrm{d}}{ }^{20}=-99.2$ (c $1.38, \mathrm{CH}_{2} \mathrm{Cl}_{2}$ ).

${ }^{1} \mathbf{H}$ NMR (400 MHz, Chloroform-d) $\delta 9.55(\mathrm{~s}, 1 \mathrm{H}), 7.42(\mathrm{t}, J=7.5 \mathrm{~Hz}, 2 \mathrm{H}), 7.36-7.23(\mathrm{~m}, 7 \mathrm{H}), 6.63$ $(\mathrm{dd}, J=15.7,10.4 \mathrm{~Hz}, 1 \mathrm{H}), 6.38(\mathrm{~d}, J=15.7 \mathrm{~Hz}, 1 \mathrm{H}), 6.21(\mathrm{dd}, J=15.1,10.4 \mathrm{~Hz}, 1 \mathrm{H}), 5.56(\mathrm{dt}, J=$ 15.1, 7.6 Hz, 1H), $2.84-2.68(\mathrm{~m}, 2 \mathrm{H}), 1.48(\mathrm{~s}, 3 \mathrm{H})$.

${ }^{13}$ C NMR (126 MHz, Chloroform-d) $\delta$ 202.0, 139.4, 135.9, 134.0, 133.0, 130.1, 129.9, 129.4, 129.1, 128.8, 127.6, 127.5, 127.3, 54.2, 39.9, 19.0.

HRMS (ESI-TOF) m/z: [M+H] ${ }^{+}$Calcd for $\mathrm{C}_{20} \mathrm{H}_{20} \mathrm{ClO} 311.1197$; Found 311.1198.

IR (film, $\mathbf{c m}^{-1}$ ): 3422, 3025, 2968, 2928, 2708, 1723, 1489, 1445, 1089, 988, 820, 760, 699.

(R,4E,6E)-2-methyl-2-phenyl-7-(4-(trifluoromethyl)phenyl)hepta-4,6-dienal (3v). 0.2 mmol

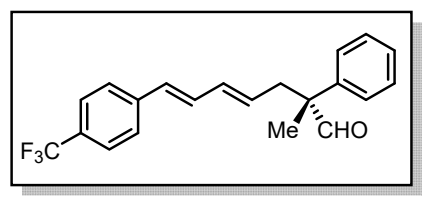
aldehyde and $0.4 \mathrm{mmol}$ cyclopropylacetylene reacted in the presence of $\mathrm{Pd}\left(\mathrm{PPh}_{3}\right)_{4}(0.01 \mathrm{mmol}), \mathbf{L 6}(0.02 \mathrm{mmol}), \mathbf{A 2}(0.12 \mathrm{mmol})$ and (S)-CPA2 $(0.02 \mathrm{mmol})$ and $3 \AA$ molecular sieves $(60 \mathrm{mg})$ in toluene $(1.0 \mathrm{~mL})$ at 100 ${ }^{\circ} \mathrm{C}$ for $48 \mathrm{~h}$ following the method $\mathrm{C}$. Flash chromatography $\left(\mathrm{SiO}_{2}\right.$, hexanes/ ethyl ether $=20 / 1$ ) of crude product gave pure $3 \mathbf{v}$ as a light yellow oil. Yield: $68.1 \mathrm{mg}, 99 \%$; $\mathrm{E} / \mathrm{Z}>20 / 1$; ee $=88 \%$, determined by HPLC (OD, hexane/isopropanol $=95 / 5$, flow rate $0.5 \mathrm{~mL} / \mathrm{min}$, $\mathrm{T}=30{ }^{\circ} \mathrm{C}, 254 \mathrm{~nm}$ ): $\mathrm{t}_{\mathrm{R}}=13.258 \mathrm{~min}$ (major), $\mathrm{t}_{\mathrm{R}}=13.947 \mathrm{~min}$ (minor). $[\alpha]_{\mathrm{d}}{ }^{20}=-87.1$ (c $1.06, \mathrm{CH}_{2} \mathrm{Cl}_{2}$ ). ${ }^{1} \mathbf{H}$ NMR (400 MHz, Chloroform-d) $\delta 9.54(\mathrm{~s}, 1 \mathrm{H}), 7.52(\mathrm{~d}, J=8.1 \mathrm{~Hz}, 2 \mathrm{H}), 7.44-7.38(\mathrm{~m}, 4 \mathrm{H})$, $7.34-7.23(\mathrm{~m}, 3 \mathrm{H}), 6.73(\mathrm{dd}, J=15.7,10.4 \mathrm{~Hz}, 1 \mathrm{H}), 6.44(\mathrm{~d}, J=15.7 \mathrm{~Hz}, 1 \mathrm{H}), 6.23$ (dd, $J=15.1$, $10.5 \mathrm{~Hz}, 1 \mathrm{H}), 5.62(\mathrm{dt}, J=15.2,7.6 \mathrm{~Hz}, 1 \mathrm{H}), 2.84-2.66(\mathrm{~m}, 2 \mathrm{H}), 1.48(\mathrm{~s}, 3 \mathrm{H})$.

${ }^{13}$ C NMR (126 MHz, Chloroform-d) $\delta$ 201.9, 140.9, 139.3, 133.8, 131.5, 131.2, 129.7, 129.5(C-F, $\left.{ }^{2} \mathrm{~J}_{\mathrm{C}-\mathrm{F}}=32.6 \mathrm{~Hz}\right), 129.2\left(\mathrm{C}-\mathrm{F},{ }^{2} \mathrm{~J}_{\mathrm{C}-\mathrm{F}}=32.6 \mathrm{~Hz}\right), 129.1,129.0\left(\mathrm{C}-\mathrm{F},{ }^{2} \mathrm{~J}_{\mathrm{C}-\mathrm{F}}=32.6 \mathrm{~Hz}\right), 128.7\left(\mathrm{C}-\mathrm{F},{ }^{2} \mathrm{~J}_{\mathrm{C}-\mathrm{F}}\right.$ $=32.6 \mathrm{~Hz}), 127.6,127.3,126.4,125.7\left(\mathrm{C}-\mathrm{F},{ }^{3} \mathrm{~J}_{\mathrm{C}-\mathrm{F}}=3.9 \mathrm{~Hz}\right), 125.7\left(\mathrm{C}-\mathrm{F},{ }^{3} \mathrm{~J}_{\mathrm{C}-\mathrm{F}}=3.9 \mathrm{~Hz}\right), 125.6(\mathrm{C}-\mathrm{F}$, $\left.{ }^{3} \mathrm{~J}_{\mathrm{C}-\mathrm{F}}=3.9 \mathrm{~Hz}\right), 125.6\left(\mathrm{C}-\mathrm{F},{ }^{3} \mathrm{~J}_{\mathrm{C}-\mathrm{F}}=3.9 \mathrm{~Hz}\right), 125.4\left(\mathrm{C}-\mathrm{F},{ }^{1} \mathrm{~J}_{\mathrm{C}-\mathrm{F}}=271.6 \mathrm{~Hz}\right), 123.3\left(\mathrm{C}-\mathrm{F},{ }^{1} \mathrm{~J}_{\mathrm{C}-\mathrm{F}}=271.6\right.$ $\mathrm{Hz}), 121.1\left(\mathrm{C}-\mathrm{F},{ }^{1} \mathrm{~J}_{\mathrm{C}-\mathrm{F}}=271.6 \mathrm{~Hz}\right), 54.2,39.9,19.1$.

${ }^{19}$ F NMR (376 MHz, Chloroform- $d$ ) $\delta-62.4$. 
HRMS (ESI-TOF) m/z: [M+H] $]^{+}$Calcd for $\mathrm{C}_{21} \mathrm{H}_{20} \mathrm{~F}_{3} \mathrm{O} 345.1461$; Found 345.1447 .

IR (film, $\mathbf{~ c m}^{-1}$ ): 3024, 2973, 2930, 2808, 2710, 1724, 1613, 1325, 1165, 1122, 1067, 989, 829, 760, 700 .

(R,4E,6E)-7-(3-fluorophenyl)-2-methyl-2-phenylhepta-4,6-dienal (3w). 0.2 mmol aldehyde and

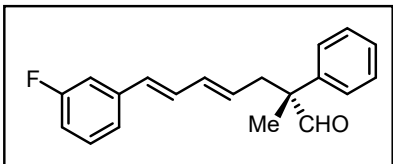
$0.4 \mathrm{mmol}$ cyclopropylacetylene reacted in the presence of $\mathrm{Pd}\left(\mathrm{PPh}_{3}\right)_{4}(0.01$ mmol), L6 (0.02 mmol), A2 (0.12 mmol) and (S)-CPA2 $(0.02 \mathrm{mmol})$ and $3 \AA$ molecular sieves $(60 \mathrm{mg})$ in toluene $(1.0 \mathrm{~mL})$ at $100{ }^{\circ} \mathrm{C}$ for $48 \mathrm{~h}$ following the method $\mathrm{C}$. Flash chromatography $\left(\mathrm{SiO}_{2}\right.$, hexanes/ ethyl ether $=20 / 1$ ) of crude product gave pure $3 \mathbf{w}$ as a light yellow oil. Yield: $52.9 \mathrm{mg}, 90 \%$; E/Z $>20 / 1$; ee $=$ $89 \%$, determined by HPLC (OD, hexane/isopropanol $=95 / 5$, flow rate $0.5 \mathrm{~mL} / \mathrm{min}, \mathrm{T}=30{ }^{\circ} \mathrm{C}, 254$ $\mathrm{nm}$ ): $\mathrm{t}_{\mathrm{R}}=14.142 \min$ (major), $\mathrm{t}_{\mathrm{R}}=15.889 \min$ (minor). $[\alpha]_{\mathrm{d}}{ }^{20}=-80.8\left(\mathrm{c} 1.20, \mathrm{CH}_{2} \mathrm{Cl}_{2}\right.$ ).

${ }^{1}$ H NMR (400 MHz, Chloroform-d) $\delta 9.52(\mathrm{~s}, 1 \mathrm{H}), 7.39(\mathrm{t}, J=7.5 \mathrm{~Hz}, 2 \mathrm{H}), 7.33-7.18(\mathrm{~m}, 4 \mathrm{H}), 7.07$ $(\mathrm{d}, J=7.8 \mathrm{~Hz}, 1 \mathrm{H}), 7.01(\mathrm{~d}, J=10.3 \mathrm{~Hz}, 1 \mathrm{H}), 6.86(\mathrm{t}, J=8.2 \mathrm{~Hz}, 1 \mathrm{H}), 6.62(\mathrm{dd}, J=15.7,10.4 \mathrm{~Hz}$, $1 \mathrm{H}), 6.37(\mathrm{~d}, J=15.7 \mathrm{~Hz}, 1 \mathrm{H}), 6.19(\mathrm{dd}, J=15.1,10.5 \mathrm{~Hz}, 1 \mathrm{H}), 5.55(\mathrm{dt}, J=15.2,7.6 \mathrm{~Hz}, 1 \mathrm{H}), 2.81$ $-2.65(\mathrm{~m}, 2 \mathrm{H}), 1.45(\mathrm{~s}, 3 \mathrm{H})$.

${ }^{13}$ C NMR (126 MHz, Chloroform- $d$ ) $\delta 202.0,164.2\left(\mathrm{C}-\mathrm{F},{ }^{1} \mathrm{~J}_{\mathrm{C}-\mathrm{F}}=245.4 \mathrm{~Hz}\right), 162.3\left(\mathrm{C}-\mathrm{F},{ }^{1} \mathrm{~J}_{\mathrm{C}-\mathrm{F}}=245.4\right.$ $\mathrm{Hz}), 139.9\left(\mathrm{C}-\mathrm{F},{ }^{3} \mathrm{~J}_{\mathrm{C}-\mathrm{F}}=7.8 \mathrm{~Hz}\right), 139.8\left(\mathrm{C}-\mathrm{F},{ }^{3} \mathrm{~J}_{\mathrm{C}-\mathrm{F}}=7.8 \mathrm{~Hz}\right), 139.4,133.9,130.6,130.1\left(\mathrm{C}-\mathrm{F},{ }^{3} \mathrm{~J}_{\mathrm{C}-\mathrm{F}}=\right.$ $7.8 \mathrm{~Hz}), 130.1\left(\mathrm{C}-\mathrm{F},{ }^{3} \mathrm{~J}_{\mathrm{C}-\mathrm{F}}=7.8 \mathrm{~Hz}\right), 130.1,130.0,129.1,127.6,127.3,122.3\left(\mathrm{C}-\mathrm{F},{ }^{4} \mathrm{~J}_{\mathrm{C}-\mathrm{F}}=2.8 \mathrm{~Hz}\right)$, $122.2\left(\mathrm{C}-\mathrm{F},{ }^{4} \mathrm{~J}_{\mathrm{C}-\mathrm{F}}=2.8 \mathrm{~Hz}\right), 114.3\left(\mathrm{C}-\mathrm{F},{ }^{2} \mathrm{~J}_{\mathrm{C}-\mathrm{F}}=21.6 \mathrm{~Hz}\right), 114.1\left(\mathrm{C}-\mathrm{F},{ }^{2} \mathrm{~J}_{\mathrm{C}-\mathrm{F}}=21.6 \mathrm{~Hz}\right), 112.7(\mathrm{C}-\mathrm{F}$, $\left.{ }^{2} \mathrm{~J}_{\mathrm{C}-\mathrm{F}}=21.6 \mathrm{~Hz}\right), 112.5\left(\mathrm{C}-\mathrm{F},{ }^{2} \mathrm{~J}_{\mathrm{C}-\mathrm{F}}=21.6 \mathrm{~Hz}\right), 54.2,39.9,19.1$.

${ }^{19}$ F NMR (471 MHz, Chloroform-d) $\delta$-113.6.

HRMS (ESI-TOF) m/z: [M+H] $]^{+}$Calcd for $\mathrm{C}_{20} \mathrm{H}_{20} \mathrm{FO} 295.1493$; Found 295.1491.

IR (film, $\mathbf{c m}^{-1}$ ): 3433, 3023, 2972, 2929, 2807, 2709, 1723, 1606, 1579, 1485, 1445, 1251,1144, 988, $777,760,699,682$.

(R,4E,6E)-7-(3-chlorophenyl)-2-methyl-2-phenylhepta-4,6-dienal (3x). 0.2 mmol aldehyde and

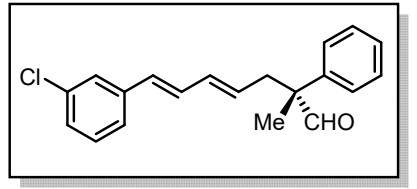
$0.4 \mathrm{mmol}$ cyclopropylacetylene reacted in the presence of $\mathrm{Pd}\left(\mathrm{PPh}_{3}\right) 4(0.01$ mmol), L6 (0.02 mmol), A2 (0.12 mmol) and (S)-CPA2 (0.02 mmol) and $3 \AA$ molecular sieves $(60 \mathrm{mg})$ in toluene $(1.0 \mathrm{~mL})$ at $100{ }^{\circ} \mathrm{C}$ for $48 \mathrm{~h}$ following the method $\mathrm{C}$. Flash chromatography $\left(\mathrm{SiO}_{2}\right.$, hexanes/ ethyl ether $=20 / 1)$ of crude product gave pure 3x as a light yellow oil. Yield: $61.4 \mathrm{mg}, 99 \%$; E/Z $>20 / 1$; ee = $90 \%$, determined by HPLC (OD, hexane/isopropanol $=95 / 5$, flow rate $0.5 \mathrm{~mL} / \mathrm{min}, \mathrm{T}=30{ }^{\circ} \mathrm{C}, 254$ $\mathrm{nm}$ ): $\mathrm{t}_{\mathrm{R}}=15.106 \mathrm{~min}$ (major), $\mathrm{t}_{\mathrm{R}}=17.058 \min$ (minor). $[\alpha]_{\mathrm{d}}{ }^{20}=-84.0\left(\mathrm{c} 1.38, \mathrm{CH}_{2} \mathrm{Cl}_{2}\right)$.

${ }^{1}$ H NMR (500 MHz, Chloroform-d) $\delta 9.52(\mathrm{~s}, 1 \mathrm{H}), 7.44-7.36(\mathrm{~m}, 2 \mathrm{H}), 7.33-7.22(\mathrm{~m}, 4 \mathrm{H}), 7.21$ $7.10(\mathrm{~m}, 3 \mathrm{H}), 6.63(\mathrm{dd}, J=15.7,10.5 \mathrm{~Hz}, 1 \mathrm{H}), 6.34(\mathrm{~d}, J=15.7 \mathrm{~Hz}, 1 \mathrm{H}), 6.18(\mathrm{dd}, J=15.1,10.4 \mathrm{~Hz}$, $1 \mathrm{H}), 5.55$ (dt, $J=14.9,7.0 \mathrm{~Hz}, 1 \mathrm{H}), 2.82-2.65(\mathrm{~m}, 2 \mathrm{H}), 1.46(\mathrm{~s}, 3 \mathrm{H})$.

${ }^{13}$ C NMR (126 MHz, Chloroform-d) $\delta$ 201.9, 139.3, 134.6, 133.9, 130.7, 130.2, 129.9, 129.7, 129.1, 127.6, 127.3, 127.3, 126.1, 124.5, 54.2, 39.9, 19.0.

HRMS (ESI-TOF) m/z: [M+H] ${ }^{+}$Calcd for $\mathrm{C}_{20} \mathrm{H}_{20} \mathrm{ClO} 311.1197$; Found 311.1198.

IR (film, $\mathbf{c m}^{-1}$ ): 3058, 3022, 2973, 2929, 2807, 2709, 1723, 1590, 1560, 1476, 1445, 1077, 988, 880, $776,761,700,682$. 
(R,4E,6E)-2-methyl-2-phenyl-7-(m-tolyl)hepta-4,6-dienal (3y). $0.2 \mathrm{mmol}$ aldehyde and $0.4 \mathrm{mmol}$

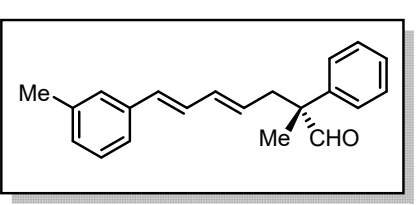
cyclopropylacetylene reacted in the presence of $\mathrm{Pd}\left(\mathrm{PPh}_{3}\right)_{4}(0.01 \mathrm{mmol})$, L6 $(0.02 \mathrm{mmol}), \mathbf{A 2}(0.12 \mathrm{mmol})$ and $(\mathrm{S})$-CPA2 $(0.02 \mathrm{mmol})$ and $3 \AA$ molecular sieves $(60 \mathrm{mg})$ in toluene $(1.0 \mathrm{~mL})$ at $100{ }^{\circ} \mathrm{C}$ for $48 \mathrm{~h}$ following the method C. Flash chromatography $\left(\mathrm{SiO}_{2}\right.$, hexanes/ ethyl ether $\left.=20 / 1\right)$ of crude product gave pure $3 \mathbf{y}$ as a light yellow oil. Yield: $50.5 \mathrm{mg}, 87 \%$; E/Z $>20 / 1$; ee $=88 \%$, determined by HPLC (OD, hexane/isopropanol $=95 / 5$, flow rate $0.5 \mathrm{~mL} / \mathrm{min}, \mathrm{T}=30{ }^{\circ} \mathrm{C}, 254 \mathrm{~nm}$ ): $\mathrm{t}_{\mathrm{R}}=15.931 \mathrm{~min}$ (major), $\mathrm{t}_{\mathrm{R}}=18.501 \mathrm{~min}$ (minor). $[\alpha]_{\mathrm{d}}{ }^{20}=-85.2\left(\mathrm{c} 1.62, \mathrm{CH}_{2} \mathrm{Cl}_{2}\right)$.

${ }^{1}$ H NMR (400 MHz, Chloroform-d) $\delta 9.52(\mathrm{~s}, 1 \mathrm{H}), 7.41$ - $7.35(\mathrm{~m}, 2 \mathrm{H}), 7.32$ - $7.23(\mathrm{~m}, 3 \mathrm{H}), 7.19$ $7.11(\mathrm{~m}, 3 \mathrm{H}), 7.02-6.97(\mathrm{~m}, 1 \mathrm{H}), 6.63(\mathrm{dd}, J=15.7,10.4 \mathrm{~Hz}, 1 \mathrm{H}), 6.38(\mathrm{~d}, J=15.7 \mathrm{~Hz}, 1 \mathrm{H}), 6.19$ (dd, $J=15.1,10.5 \mathrm{~Hz}, 1 \mathrm{H}), 5.50$ (dt, $J=15.1,7.6 \mathrm{~Hz}, 1 \mathrm{H}), 2.80-2.66$ (m, 2H), 2.30 (s, 3H), 1.45 (s, $3 \mathrm{H})$.

${ }^{13}$ C NMR (126 MHz, Chloroform-d) $\delta$ 202.1, 139.5, 137.3, 134.6, 134.5, 131.3, 129.4, 129.0, 128.7, $127.9,127.5,127.3,126.3,54.2,39.9,21.3,19.1$.

HRMS (ESI-TOF) m/z: [M+H] $]^{+}$Calcd for $\mathrm{C}_{21} \mathrm{H}_{23} \mathrm{O}$ 291.1743; Found 291.1756.

IR (film, $\mathbf{c m}^{-1}$ ): 3021, 2971, 2920, 2806, 2708, 1724, 1599, 1494, 1445, 988, 775, 761, 699.

(R,4E,6E)-7-(3-methoxyphenyl)-2-methyl-2-phenylhepta-4,6-dienal (3z). $0.2 \mathrm{mmol}$ aldehyde and

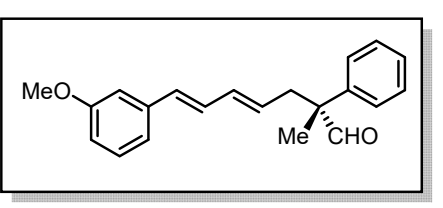
$0.4 \mathrm{mmol}$ cyclopropylacetylene reacted in the presence of $\mathrm{Pd}\left(\mathrm{PPh}_{3}\right)_{4}$ (0.01 mmol), L6 (0.02 mmol), A2 $(0.12 \mathrm{mmol})$ and (S)-CPA2 (0.02 $\mathrm{mmol})$ and $3 \AA$ molecular sieves $(60 \mathrm{mg})$ in toluene $(1.0 \mathrm{~mL})$ at $100{ }^{\circ} \mathrm{C}$ for $48 \mathrm{~h}$ following the method $\mathrm{C}$. Flash chromatography $\left(\mathrm{SiO}_{2}\right.$, hexanes/ ethyl ether $=15 / 1)$ of crude product gave pure $\mathbf{3 z}$ as a light yellow oil. Yield: $44.1 \mathrm{mg}, 72 \%$; E/Z > 20/1; ee $=87 \%$, determined by HPLC (OD, hexane/isopropanol $=95 / 5$, flow rate $0.5 \mathrm{~mL} / \mathrm{min}, \mathrm{T}=$ $30{ }^{\circ} \mathrm{C}, 254 \mathrm{~nm}$ ): $\mathrm{t}_{\mathrm{R}}=24.413 \mathrm{~min}$ (major), $\mathrm{t}_{\mathrm{R}}=28.213 \mathrm{~min}$ (minor). $[\alpha]_{\mathrm{d}}{ }^{20}=-86.0$ (c 1.64, $\mathrm{CH}_{2} \mathrm{Cl}_{2}$ ).

${ }^{1} \mathbf{H}$ NMR (500 MHz, Chloroform- $\left.d\right) \delta 9.52(\mathrm{~s}, 1 \mathrm{H}), 7.39(\mathrm{t}, J=7.7 \mathrm{~Hz}, 2 \mathrm{H}), 7.31-7.23(\mathrm{~m}, 3 \mathrm{H}), 7.18$ $(\mathrm{t}, J=7.9 \mathrm{~Hz}, 1 \mathrm{H}), 6.92(\mathrm{~d}, J=7.7 \mathrm{~Hz}, 1 \mathrm{H}), 6.86(\mathrm{~s}, 1 \mathrm{H}), 6.74(\mathrm{dd}, J=8.2,2.6 \mathrm{~Hz}, 1 \mathrm{H}), 6.63(\mathrm{dd}, J$ $=15.6,10.4 \mathrm{~Hz}, 1 \mathrm{H}), 6.39(\mathrm{~d}, J=15.7 \mathrm{~Hz}, 1 \mathrm{H}), 6.19(\mathrm{dd}, J=15.1,10.4 \mathrm{~Hz}, 1 \mathrm{H}), 5.52(\mathrm{dt}, J=15.1$, $7.6 \mathrm{~Hz}, 1 \mathrm{H}), 3.78$ (s, 3H), $2.79-2.67$ (m, 2H), 1.45 (s, 3H).

${ }^{13}$ C NMR (126 MHz, Chloroform-d) $\delta$ 202.1, 159.9, 139.5, 138.9, 134.2, 131.2, 129.7, 129.6, 129.1, 129.1, 127.6, 127.3, 119.1, 113.3, 111.4, 55.3, 54.2, 39.9, 19.1.

HRMS (ESI-TOF) m/z: [M+H] $]^{+}$Calcd for $\mathrm{C}_{21} \mathrm{H}_{23} \mathrm{O}_{2}$ 307.1693; Found 307.1685.

IR (film, $\mathbf{c m}^{-1}$ ): 3425, 3022, 2935, 2834, 2709, 1723, 1596, 1576, 1493, 1266, 1157, 1046, 989, 760, 700,688 .

(R,4E,6E)-7-(2-fluorophenyl)-2-methyl-2-phenylhepta-4,6-dienal (3aa). 0.2 mmol aldehyde and

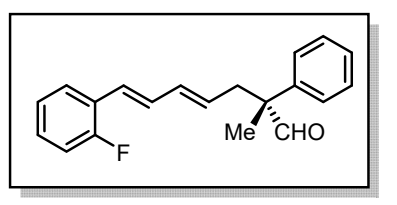
$0.4 \mathrm{mmol}$ cyclopropylacetylene reacted in the presence of $\mathrm{Pd}\left(\mathrm{PPh}_{3}\right)_{4}(0.01$ mmol), L6 (0.02 mmol), A2 (0.12 mmol) and (S)-CPA2 $(0.02 \mathrm{mmol})$ and $3 \AA$ molecular sieves $(60 \mathrm{mg})$ in toluene $(1.0 \mathrm{~mL})$ at $100{ }^{\circ} \mathrm{C}$ for $48 \mathrm{~h}$ following the method $\mathrm{C}$. Flash chromatography $\left(\mathrm{SiO}_{2}\right.$, hexanes/ ethyl ether $=20 / 1$ ) of crude product gave pure 3aa as a light yellow oil. Yield: $51.2 \mathrm{mg}, 87 \%$; E/Z $>20 / 1$; ee = $86 \%$, determined by HPLC (OD, hexane/isopropanol $=95 / 5$, flow rate $0.5 \mathrm{~mL} / \mathrm{min}, \mathrm{T}=30{ }^{\circ} \mathrm{C}, 254$ $\mathrm{nm}$ ): $\mathrm{t}_{\mathrm{R}}=14.449 \mathrm{~min}$ (major), $\mathrm{t}_{\mathrm{R}}=16.310 \mathrm{~min}$ (minor). $[\alpha]_{\mathrm{d}}{ }^{20}=-98.9\left(\mathrm{c} 1.12, \mathrm{CH}_{2} \mathrm{Cl}_{2}\right.$ ). 
${ }^{1}$ H NMR (500 MHz, Chloroform-d) $\delta 9.54(\mathrm{~s}, 1 \mathrm{H}), 7.42-7.37(\mathrm{~m}, 3 \mathrm{H}), 7.34-7.25(\mathrm{~m}, 3 \mathrm{H}), 7.18-$ $7.13(\mathrm{~m}, 1 \mathrm{H}), 7.07-6.97(\mathrm{~m}, 2 \mathrm{H}), 6.72(\mathrm{dd}, J=15.9,10.4 \mathrm{~Hz}, 1 \mathrm{H}), 6.58(\mathrm{~d}, J=15.9 \mathrm{~Hz}, 1 \mathrm{H}), 6.23$ $(\mathrm{dd}, J=15.1,10.4 \mathrm{~Hz}, 1 \mathrm{H}), 5.57$ (dt, $J=15.1,7.5 \mathrm{~Hz}, 1 \mathrm{H}), 2.81-2.67(\mathrm{~m}, 2 \mathrm{H}), 1.47(\mathrm{~s}, 3 \mathrm{H})$.

${ }^{13} \mathrm{C}$ NMR $\left(126 \mathrm{MHz}\right.$, Chloroform-d) $\delta 202.0,161.3\left(\mathrm{C}-\mathrm{F},{ }^{1} \mathrm{~J}_{\mathrm{C}-\mathrm{F}}=249.6 \mathrm{~Hz}\right), 159.3\left(\mathrm{C}-\mathrm{F},{ }^{1} \mathrm{~J}_{\mathrm{C}-\mathrm{F}}=\right.$ $249.6 \mathrm{~Hz}), 139.4,134.5,131.2\left(\mathrm{C}-\mathrm{F},{ }^{3} \mathrm{~J}_{\mathrm{C}-\mathrm{F}}=5.1 \mathrm{~Hz}\right), 131.1\left(\mathrm{C}-\mathrm{F},{ }^{3} \mathrm{~J}_{\mathrm{C}-\mathrm{F}}=5.1 \mathrm{~Hz}\right), 130.3,129.1$, $128.6\left(\mathrm{C}-\mathrm{F},{ }^{3} \mathrm{~J}_{\mathrm{C}-\mathrm{F}}=8.3 \mathrm{~Hz}\right), 128.6\left(\mathrm{C}-\mathrm{F},{ }^{3} \mathrm{~J}_{\mathrm{C}-\mathrm{F}}=8.3 \mathrm{~Hz}\right), 127.6,127.3,127.0\left(\mathrm{C}-\mathrm{F},{ }^{3} \mathrm{~J}_{\mathrm{C}-\mathrm{F}}=3.7 \mathrm{~Hz}\right)$, $127.0\left(\mathrm{C}-\mathrm{F},{ }^{4} \mathrm{~J}_{\mathrm{C}-\mathrm{F}}=3.7 \mathrm{~Hz}\right), 125.3\left(\mathrm{C}-\mathrm{F},{ }^{2} \mathrm{~J}_{\mathrm{C}-\mathrm{F}}=11.9 \mathrm{~Hz}\right), 125.2\left(\mathrm{C}-\mathrm{F},{ }^{2} \mathrm{~J}_{\mathrm{C}-\mathrm{F}}=11.9 \mathrm{~Hz}\right), 124.2(\mathrm{C}-\mathrm{F}$, $\left.{ }^{3} \mathrm{~J}_{\mathrm{C}-\mathrm{F}}=3.7 \mathrm{~Hz}\right), 124.2\left(\mathrm{C}-\mathrm{F},{ }^{4} \mathrm{~J}_{\mathrm{C}-\mathrm{F}}=3.7 \mathrm{~Hz}\right), 123.6\left(\mathrm{C}-\mathrm{F},{ }^{4} \mathrm{~J}_{\mathrm{C}-\mathrm{F}}=3.2 \mathrm{~Hz}\right), 123.5\left(\mathrm{C}-\mathrm{F},{ }^{4} \mathrm{~J}_{\mathrm{C}-\mathrm{F}}=3.2 \mathrm{~Hz}\right)$, $115.9\left(\mathrm{C}-\mathrm{F},{ }^{2} \mathrm{~J}_{\mathrm{C}-\mathrm{F}}=22.1 \mathrm{~Hz}\right), 115.7\left(\mathrm{C}-\mathrm{F},{ }^{2} \mathrm{~J}_{\mathrm{C}-\mathrm{F}}=22.1 \mathrm{~Hz}\right), 54.2,39.9,19.1$.

${ }^{19}$ F NMR (376 MHz, Chloroform-d) $\delta-118.1$.

HRMS (ESI-TOF) m/z: [M+H] $]^{+}$Calcd for $\mathrm{C}_{20} \mathrm{H}_{20} \mathrm{FO} 295.1493$; Found 295.1483.

IR (film, $\mathbf{c m}^{-1}$ ): 3025, 2965, 2920, 2849, 2804, 2708, 1723, 1484, 1228, 1088, 991, 754, 699.

(R,4E,6E)-7-(2-methoxyphenyl)-2-methyl-2-phenylhepta-4,6-dienal (3ab). 0.2 mmol aldehyde

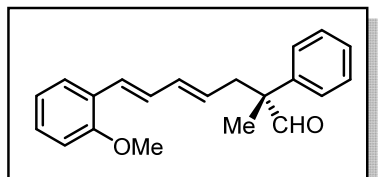
and $1.0 \mathrm{mmol}$ cyclopropylacetylene reacted in the presence of $\mathrm{Pd}\left(\mathrm{PPh}_{3}\right)_{4}$ (0.01 mmol), L6 (0.02 mmol), A2 (0.12 mmol) and (S)-CPA2 $(0.02 \mathrm{mmol})$ and $3 \AA$ molecular sieves $(60 \mathrm{mg})$ in toluene $(1.0 \mathrm{~mL})$ at $100{ }^{\circ} \mathrm{C}$ for $48 \mathrm{~h}$ following the method $\mathrm{C}$. Flash chromatography $\left(\mathrm{SiO}_{2}\right.$, hexanes/ ethyl ether $=15 / 1$ ) of crude product gave pure 3ab as a light yellow oil. Yield: $25.7 \mathrm{mg}, 42 \%$; E/Z $>20 / 1$; ee = $83 \%$, determined by HPLC (OD, hexane/isopropanol = 95/5, flow rate $0.5 \mathrm{~mL} / \mathrm{min}, \mathrm{T}=30{ }^{\circ} \mathrm{C}, 254$ $\mathrm{nm}$ ): $\mathrm{t}_{\mathrm{R}}=37.731 \mathrm{~min}$ (major), $\mathrm{t}_{\mathrm{R}}=50.222 \mathrm{~min}$ (minor). $[\alpha]_{\mathrm{d}}{ }^{20}=-84.0\left(\mathrm{c} 1.00, \mathrm{CH}_{2} \mathrm{Cl}_{2}\right)$.

${ }^{1}$ H NMR (400 MHz, Chloroform-d) $\delta 9.52(\mathrm{~s}, 1 \mathrm{H}), 7.42-7.33(\mathrm{~m}, 3 \mathrm{H}), 7.32-7.22(\mathrm{~m}, 4 \mathrm{H}), 7.16$ (ddd, $J=8.2,7.3,1.7 \mathrm{~Hz}, 1 \mathrm{H}), 6.90-6.80(\mathrm{~m}, 2 \mathrm{H}), 6.76(\mathrm{~d}, J=15.9 \mathrm{~Hz}, 1 \mathrm{H}), 6.66$ (dd, $J=15.9,9.9$ $\mathrm{Hz}, 1 \mathrm{H}), 6.24$ (ddt, $J=15.0,10.0,1.3 \mathrm{~Hz}, 1 \mathrm{H}), 5.49$ (dt, $J=15.1,7.6 \mathrm{~Hz}, 1 \mathrm{H}), 3.82$ (s, 3H), $2.81-$ $2.64(\mathrm{~m}, 2 \mathrm{H}), 1.44(\mathrm{~s}, 3 \mathrm{H})$.

${ }^{13}$ C NMR (126 MHz, Chloroform-d) $\delta$ 202.2, 156.8, 139.6, 135.2, 129.5, 129.0, 128.6, 128.5, 127.5, $127.3,126.5,126.4,126.2,120.8,111.0,55.6,54.2,39.9,19.1$.

HRMS (ESI-TOF) m/z: [M+H] $]^{+}$Calcd for $\mathrm{C}_{21} \mathrm{H}_{23} \mathrm{O}_{2}$ 307.1693; Found 307.1689.

IR (film, $\mathbf{c m}^{-1}$ ): 3203, 2962, 2920, 2849, 2708, 1723, 1487, 1463, 1243, 1028, 992, 751, 700.

(R,4E,6E)-2-methyl-2-phenyl-7-(thiophen-3-yl)hepta-4,6-dienal (3ac). $0.2 \mathrm{mmol}$ aldehyde and

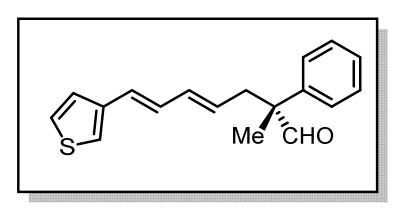
$0.4 \mathrm{mmol}$ cyclopropylacetylene reacted in the presence of $\mathrm{Pd}\left(\mathrm{PPh}_{3}\right)_{4}(0.01$ mmol), L6 (0.02 mmol), A2 (0.12 mmol) and (S)-CPA2 (0.02 mmol) and $3 \AA$ molecular sieves $(60 \mathrm{mg})$ in toluene $(1.0 \mathrm{~mL})$ at $100^{\circ} \mathrm{C}$ for $48 \mathrm{~h}$ following the method C. Flash chromatography $\left(\mathrm{SiO}_{2}\right.$, hexanes/ ethyl ether $\left.=15 / 1\right)$ of crude product gave pure 3ac as a light yellow oil. Yield: $51.3 \mathrm{mg}, 91 \%$; E/Z $>20 / 1$; ee $=88 \%$, determined by HPLC (OD, hexane/isopropanol $=95 / 5$, flow rate $\left.0.5 \mathrm{~mL} / \mathrm{min}, \mathrm{T}=30{ }^{\circ} \mathrm{C}, 254 \mathrm{~nm}\right)$ : $\mathrm{t}_{\mathrm{R}}=17.533 \mathrm{~min}$ (major), $\mathrm{t}_{\mathrm{R}}=20.436 \mathrm{~min}$ (minor). $[\alpha]_{\mathrm{d}}{ }^{20}=-106.9\left(\mathrm{c} 1.50, \mathrm{CH}_{2} \mathrm{Cl}_{2}\right)$.

${ }^{1} \mathbf{H}$ NMR (500 MHz, Chloroform-d) $\delta 9.52(\mathrm{~s}, 1 \mathrm{H}), 7.38(\mathrm{t}, J=7.6 \mathrm{~Hz}, 2 \mathrm{H}), 7.33-7.20(\mathrm{~m}, 5 \mathrm{H}), 7.15$ $(\mathrm{d}, J=5.1 \mathrm{~Hz}, 1 \mathrm{H}), 7.08(\mathrm{~d}, J=2.9 \mathrm{~Hz}, 1 \mathrm{H}), 6.52-6.39(\mathrm{~m}, 2 \mathrm{H}), 6.15(\mathrm{dd}, J=15.1,9.5 \mathrm{~Hz}, 1 \mathrm{H})$, $5.48(\mathrm{dt}, J=15.1,7.5 \mathrm{~Hz}, 1 \mathrm{H}), 2.78-2.65(\mathrm{~m}, 2 \mathrm{H}), 1.45(\mathrm{~s}, 3 \mathrm{H})$.

${ }^{13}$ C NMR (126 MHz, Chloroform-d) $\delta$ 202.1, 140.2, 139.5, 134.2, 129.0, 128.9, 128.8, 127.5, 127.3, $126.1,125.5,124.9,121.9,54.2,39.9,19.1$.

HRMS (ESI-TOF) m/z: [M+H] $]^{+}$Calcd for $\mathrm{C}_{18} \mathrm{H}_{19} \mathrm{OS} 283.1151$; Found 283.1147.

IR (film, $\mathbf{c m}^{-1}$ ): 3421, 3022, 2975, 2920, 2848, 2807, 2708, 1721, 1495, 1445, 987, 762, 699, 624. 


\section{Synthetic Applications}
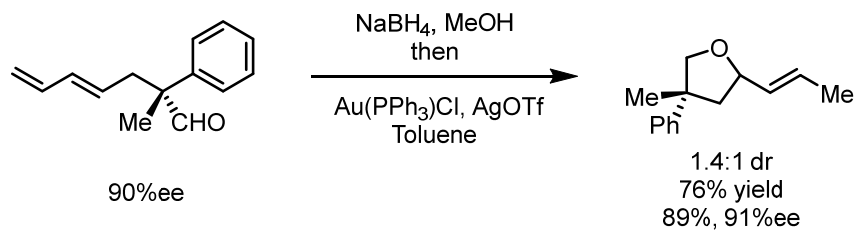

$\mathrm{NaBH}_{4}(15.2 \mathrm{mg}, 0.4 \mathrm{mmol})$ was added to a solution of aldehyde $(20.0 \mathrm{mg}, 0.1 \mathrm{mmol})$ in methanol $(0.5 \mathrm{~mL})$ at $0^{\circ} \mathrm{C}$. The mixture was stirred at this temperature for $0.5 \mathrm{~h}$ and then $\mathrm{H}_{2} \mathrm{O}(2 \mathrm{~mL})$ was added slowly. The mixture was extracted with ethyl acetate $(3 \times 4 \mathrm{~mL})$. The combined organic layers were dried over anhydrous $\mathrm{Na}_{2} \mathrm{SO}_{4}$. The mixture was filtered and concentrated in vacuo. The residue was purified by flash chromatography $\left(\mathrm{SiO}_{2}\right.$, hexanes/ ethyl ether $\left.=6 / 1\right)$ to give the alcohol. A mixture of $\left(\mathrm{PPh}_{3}\right) \mathrm{AuCl}(2.5 \mathrm{mg}, 0.005 \mathrm{mmol})$ and $\mathrm{AgOTf}(1.3 \mathrm{mg}, 0.005 \mathrm{mmol})$ in anhydrous toluene $(0.2 \mathrm{~mL})$ was stirred at room temperature for $10 \mathrm{~min}$, treated with a solution of the alcohol in toluene $(0.8 \mathrm{~mL})$, and the resulting suspension was stirred at $60^{\circ} \mathrm{C}$ for $12 \mathrm{~h}$. Progress of the reaction was monitored by TLC. Upon the completion of the reaction, solvent was evaporated. The residue was purified by flash chromatography $\left(\mathrm{SiO}_{2}\right.$, hexanes/ ethyl ether $\left.=20 / 1\right)$ to provide the product $(76 \%$ yield, $15.2 \mathrm{mg})$.

\section{(2S,4R)-4-methyl-4-phenyl-2-((E)-prop-1-en-1-yl)tetrahydrofuran (4-major)}

Colorless oil, yield: $8.6 \mathrm{mg}, 43 \%$; ee $=89 \%$, determined by HPLC (IB,

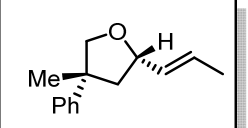
hexane/isopropanol $=98 / 2$, flow rate $\left.0.4 \mathrm{~mL} / \mathrm{min}, \mathrm{T}=30{ }^{\circ} \mathrm{C}, 210 \mathrm{~nm}\right): \mathrm{t}_{\mathrm{R}}=10.517$ $\min$ (major), $\mathrm{t}_{\mathrm{R}}=11.366 \mathrm{~min}$ (minor). $[\alpha]_{\mathrm{d}}{ }^{20}=-18.9\left(\mathrm{c} 0.65, \mathrm{CH}_{2} \mathrm{Cl}_{2}\right.$ ).

${ }^{1} \mathbf{H}$ NMR (400 MHz, Chloroform-d) $\delta 7.36-7.28(\mathrm{~m}, 2 \mathrm{H}), 7.26-7.15(\mathrm{~m}, 3 \mathrm{H})$, $5.74(\mathrm{dq}, J=15.1,6.5 \mathrm{~Hz}, 1 \mathrm{H}), 5.51$ (ddq, $J=15.2,7.6,1.7 \mathrm{~Hz}, 1 \mathrm{H}), 4.59$ (dt, $J=9.2,6.6 \mathrm{~Hz}, 1 \mathrm{H})$, $4.02-3.94(\mathrm{~m}, 2 \mathrm{H}), 2.23(\mathrm{dd}, J=12.2,6.1 \mathrm{~Hz}, 1 \mathrm{H}), 2.00(\mathrm{dd}, J=12.2,9.7 \mathrm{~Hz}, 1 \mathrm{H}), 1.70(\mathrm{dd}, J=$ $6.5,1.6 \mathrm{~Hz}, 3 \mathrm{H}), 1.44(\mathrm{~s}, 3 \mathrm{H})$.

${ }^{13}$ C NMR (126 MHz, Chloroform-d) $\delta$ 147.7, 132.2, 128.6, 128.1, 126.3, 126.0, 80.0, 78.9, 48.0, 46.1, 28.2, 17.8 .

HRMS (ESI-TOF) m/z: [M+H $]^{+}$Calcd for $\mathrm{C}_{14} \mathrm{H}_{19} \mathrm{O}$ 203.1430; Found 203.1432.

IR (film, $\mathbf{c m}^{-1}$ ): 3397, 2962, 2920, 2854, 1496, 1446, 1047, 963, 762, 700, 545.

\section{(2R,4R)-4-methyl-4-phenyl-2-((E)-prop-1-en-1-yl)tetrahydrofuran (4-minor)}

Colorless oil, yield: $6.6 \mathrm{mg}, 33 \%$; ee $=91 \%$, determined by HPLC (IF,

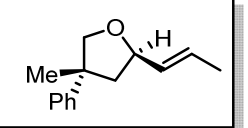
hexane/isopropanol $=98 / 2$, flow rate $\left.0.4 \mathrm{~mL} / \mathrm{min}, \mathrm{T}=30{ }^{\circ} \mathrm{C}, 210 \mathrm{~nm}\right): \mathrm{t}_{\mathrm{R}}=12.866$ $\min$ (major), $\mathrm{t}_{\mathrm{R}}=13.643 \mathrm{~min}$ (minor). $[\alpha]_{\mathrm{d}}^{20}=27.2\left(\mathrm{c} 0.50, \mathrm{CH}_{2} \mathrm{Cl}_{2}\right)$.

${ }^{1}$ H NMR (400 MHz, Chloroform-d) $\delta 7.39-7.28(\mathrm{~m}, 4 \mathrm{H}), 7.25-7.18(\mathrm{~m}, 1 \mathrm{H})$, $5.77-5.51(\mathrm{~m}, 2 \mathrm{H}), 4.37(\mathrm{q}, J=7.6 \mathrm{~Hz}, 1 \mathrm{H}), 4.04(\mathrm{~d}, J=8.5 \mathrm{~Hz}, 1 \mathrm{H}), 3.91(\mathrm{~d}, J=8.4 \mathrm{~Hz}, 1 \mathrm{H}), 2.42$ $(\mathrm{dd}, J=12.6,7.7 \mathrm{~Hz}, 1 \mathrm{H}), 1.83(\mathrm{dd}, J=12.6,7.8 \mathrm{~Hz}, 1 \mathrm{H}), 1.72(\mathrm{~d}, J=6.2 \mathrm{~Hz}, 3 \mathrm{H}), 1.45$ (s, 3H).

${ }^{13}$ C NMR (126 MHz, Chloroform-d) $\delta 147.5,132.5,128.6,128.2,126.2,126.1,80.3,79.0,47.9,46.8$, 28.6, 17.8 .

HRMS (ESI-TOF) m/z: [M+H] $]^{+}$Calcd for $\mathrm{C}_{14} \mathrm{H}_{19} \mathrm{O}$ 203.1430; Found 203.1432.

IR (film, $\mathbf{c m}^{-1}$ ): 3380, 2963, 2927, 2856, 1496, 1446, 1048, 963, 762, 700, 545. 

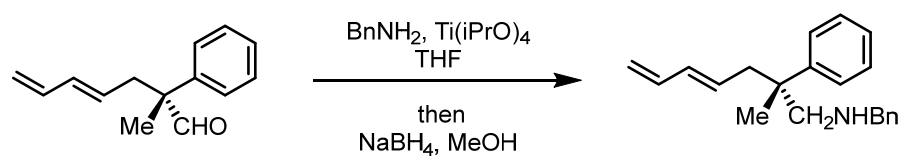

$90 \%$ yield, $88 \%$ ee

Aldehyde (20 mg, $0.1 \mathrm{mmol}, 1.0$ equiv.) was added to a flame-dried vial (10 mL) with a magnetic stirring bar. Under inert atmosphere, THF $(0.60 \mathrm{~mL})$ and amine $(0.12 \mathrm{~mol}, 1.2$ equiv. $)$ were added and the resulting solution was cooled down to $0^{\circ} \mathrm{C}$. $\mathrm{Ti}(\mathrm{iPrO})_{4}(0.12 \mathrm{mmol}, 1.2$ equiv. $)$ was added via a syringe at $0^{\circ} \mathrm{C}$, and the reaction mixture was warmed up to $25^{\circ} \mathrm{C}$ and stirred for $3 \mathrm{~h}$. Then, $\mathrm{NaBH}_{4}$ $(0.15 \mathrm{mmol}, 1.5$ equiv. $)$ and methanol $(0.60 \mathrm{~mL})$ were added, and the mixture was stirred at room temperature for additional $1 \mathrm{~h}$. The mixture was then diluted with $\mathrm{CH}_{2} \mathrm{Cl}_{2}(5.0 \mathrm{~mL})$ and treated with aqueous $\mathrm{NaOH}$ solution $(2 \mathrm{~N}, 5.0 \mathrm{~mL}$ ) for $5 \mathrm{~min}$. The layers were separated, and the aqueous layer was extracted with $\mathrm{CH}_{2} \mathrm{Cl}_{2}(3 \times 5.0 \mathrm{~mL})$. The combined organic layers were washed with brine (3.0 $\mathrm{mL}$ ), dried over $\mathrm{Na}_{2} \mathrm{SO}_{4}$ and concentrated in vacuo. The residue was purified by flash chromatography $\left(\mathrm{SiO}_{2}\right.$, hexanes/ ethyl ether $\left.=15 / 1\right)$ to provide the product $(90 \%$ yield, $26.2 \mathrm{mg})$ as a colorless oil.

\section{(R,E)-N-benzyl-2-methyl-2-phenylhepta-4,6-dien-1-amine (5)}

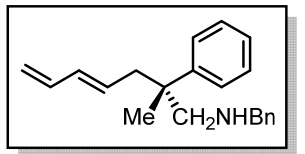

Colorless oil, yield: $26.2 \mathrm{mg}, 90 \%$; ee $=88 \%$, determined by HPLC (OJ, hexane/isopropanol $=95 / 5$, flow rate $\left.0.5 \mathrm{~mL} / \mathrm{min}, \mathrm{T}=30{ }^{\circ} \mathrm{C}, 220 \mathrm{~nm}\right): \mathrm{t}_{\mathrm{R}}=14.003$ $\min$ (major), $\mathrm{t}_{\mathrm{R}}=11.437 \mathrm{~min}$ (minor). $[\alpha]_{\mathrm{d}}{ }^{20}=-27.9\left(\mathrm{c} 1.12, \mathrm{CH}_{2} \mathrm{Cl}_{2}\right)$.

${ }^{1}$ H NMR (400 MHz, Chloroform-d) $\delta 7.29-7.20(\mathrm{~m}, 6 \mathrm{H}), 7.20-7.12(\mathrm{~m}, 4 \mathrm{H})$, $6.15(\mathrm{dt}, J=16.9,10.3 \mathrm{~Hz}, 1 \mathrm{H}), 5.98(\mathrm{dd}, J=15.1,10.4 \mathrm{~Hz}, 1 \mathrm{H}), 5.39(\mathrm{dt}, J=15.0,7.4 \mathrm{~Hz}, 1 \mathrm{H}), 5.00$ $(\mathrm{d}, J=16.5 \mathrm{~Hz}, 1 \mathrm{H}), 4.88(\mathrm{~d}, J=9.8 \mathrm{~Hz}, 1 \mathrm{H}), 3.70-3.61(\mathrm{~m}, 2 \mathrm{H}), 2.78(\mathrm{~d}, J=11.4 \mathrm{~Hz}, 1 \mathrm{H}), 2.62$ $(\mathrm{d}, J=11.4 \mathrm{~Hz}, 1 \mathrm{H}), 2.51(\mathrm{dd}, J=13.9,6.7 \mathrm{~Hz}, 1 \mathrm{H}), 2.35(\mathrm{dd}, J=14.0,8.2 \mathrm{~Hz}, 1 \mathrm{H}), 1.30(\mathrm{~s}, 3 \mathrm{H})$.

${ }^{13}$ C NMR (126 MHz, Chloroform-d) $\delta 146.1,140.7,137.3,133.7,131.4,128.4,128.4,128.0,126.9$, 126.6, 126.1, 115.2, 59.9, 54.3, 43.7, 42.3, 23.7.

HRMS (ESI-TOF) m/z: [M+ Na $]^{+}$Calcd for $\mathrm{C}_{21} \mathrm{H}_{25} \mathrm{NNa} 314.1879$; Found 314.1887.

IR (film, $\mathbf{c m}^{-1}$ ): 3349, 3085, 3060, 3026, 2965, 2919, 2848, 1601, 1495, 1454, 1117, 1005, 898, 765, $736,698$.
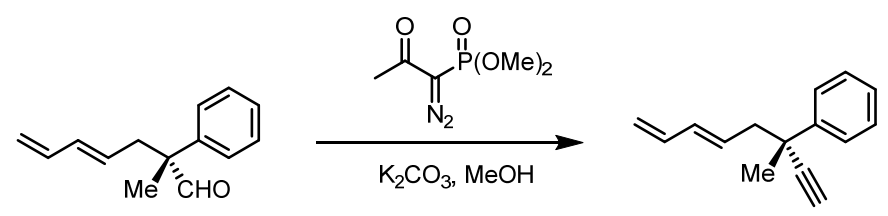

$80 \%$ yield, $90 \%$ ee

To a flame-dried vial $(10 \mathrm{~mL})$, aldehyde ( $20 \mathrm{mg}, 0.1 \mathrm{mmol}, 1.0$ equiv.), and $\mathrm{K}_{2} \mathrm{CO}_{3}(41.5 \mathrm{mg}, 0.3$ mmol, 3.0 equiv.) were added with a magnetic stirring bar. Then, methanol $(2.0 \mathrm{~mL})$ was added and followed by the addition of Ohira-Bestmann reagent $(0.15 \mathrm{mmol}, 1.5$ equiv. $)$. The reaction mixture was vigorously stirred at $25^{\circ} \mathrm{C}$ for $5 \mathrm{~h}$. The mixture was then diluted with diethyl ether $(5.0 \mathrm{~mL})$ and water $(5.0 \mathrm{~mL})$. The layers were separated, and the aqueous layer was extracted with diethyl ether (3 x $5.0 \mathrm{~mL})$. The combined organic layers were washed with brine $(10 \mathrm{~mL})$, dried over $\mathrm{Na}_{2} \mathrm{SO}_{4}$, and concentrated in vacuo. The residue was purified by flash chromatography ( $\mathrm{SiO}_{2}$, hexanes) to provide the product $(80 \%$ yield, $15.7 \mathrm{mg}$ ) as a colorless oil.

(S,E)-(3-methylocta-5,7-dien-1-yn-3-yl)benzene (6) 
Colorless oil, yield: $15.7 \mathrm{mg}, 80 \%$; ee $=90 \%$, determined by HPLC (IC, hexane/isopropanol $=100 / 0$, flow rate $\left.0.5 \mathrm{~mL} / \mathrm{min}, \mathrm{T}=30^{\circ} \mathrm{C}, 220 \mathrm{~nm}\right): \mathrm{t}_{\mathrm{R}}=8.648$ $\min$ (major), $\mathrm{t}_{\mathrm{R}}=8.159 \mathrm{~min}$ (minor). $[\alpha]_{\mathrm{d}}{ }^{20}=9.3\left(\mathrm{c} 1.07, \mathrm{CH}_{2} \mathrm{Cl}_{2}\right.$ ).

${ }^{1}$ H NMR (400 MHz, Chloroform- $d$ ) $\delta 7.52(\mathrm{~d}, J=7.5 \mathrm{~Hz}, 2 \mathrm{H}), 7.33(\mathrm{t}, J=7.5$ $\mathrm{Hz}, 2 \mathrm{H}), 7.25-7.20$ (m, 2H), 6.27 (dt, $J=16.9,10.2 \mathrm{~Hz}, 1 \mathrm{H}), 6.05(\mathrm{dd}, J=15.2,10.4 \mathrm{~Hz}, 1 \mathrm{H}), 5.65$ $(\mathrm{dt}, J=15.0,7.4 \mathrm{~Hz}, 1 \mathrm{H}), 5.09(\mathrm{~d}, J=16.9 \mathrm{~Hz}, 1 \mathrm{H}), 4.97(\mathrm{~d}, J=10.1 \mathrm{~Hz}, 1 \mathrm{H}), 2.67-2.48(\mathrm{~m}, 2 \mathrm{H})$, $2.41(\mathrm{~s}, 1 \mathrm{H}), 1.58(\mathrm{~s}, 3 \mathrm{H})$.

${ }^{13}$ C NMR (101 MHz, Chloroform-d) $\delta 144.7,137.1,134.2,130.5,128.4,126.7,126.2,115.9,89.2$, 71.8, 47.3, 40.4, 29.0 .

HRMS (ESI-TOF) m/z: [M+H] $]^{+}$Calcd for $\mathrm{C}_{15} \mathrm{H}_{17}$ 197.1325; Found 197.1319.

IR (film, $\mathbf{c m}^{-1}$ ): 3301, 2975, 2929, 1601, 1495, 1446, 1004, 901, 762, 698, 639.

[1]Ye, S.; Yu, Z.-X.; Org. Lett. 2010, 12, 804-807.

[2]Jang, Y. H.; Youn. S. W; Org. Lett. 2014, 16, 3720-3723.

[3]Y. L. Su; L. L. Li; X. L. Zhou; Z. Y. Dai; P. S. Wang; L. Z. Gong; Org. Lett. 2018, 20, 2403-2406.

[4] Guimond, N.; MacDonald, M. J.; Lemieux, V.; Beauchemin, A. M.; J. Am. Chem. Soc. 2012, 134, $16571-16577$.

[5] Hoffmann, S.; Nicoletti, M.; List, B.; J. Am. Chem. Soc. 2006, 128, 13074-13075. 


\section{NMR spectra}

(R,E)-2-methyl-2-phenylhepta-4,6-dienal (3a)

${ }^{1}$ H NMR (500 MHz, Chloroform-d)

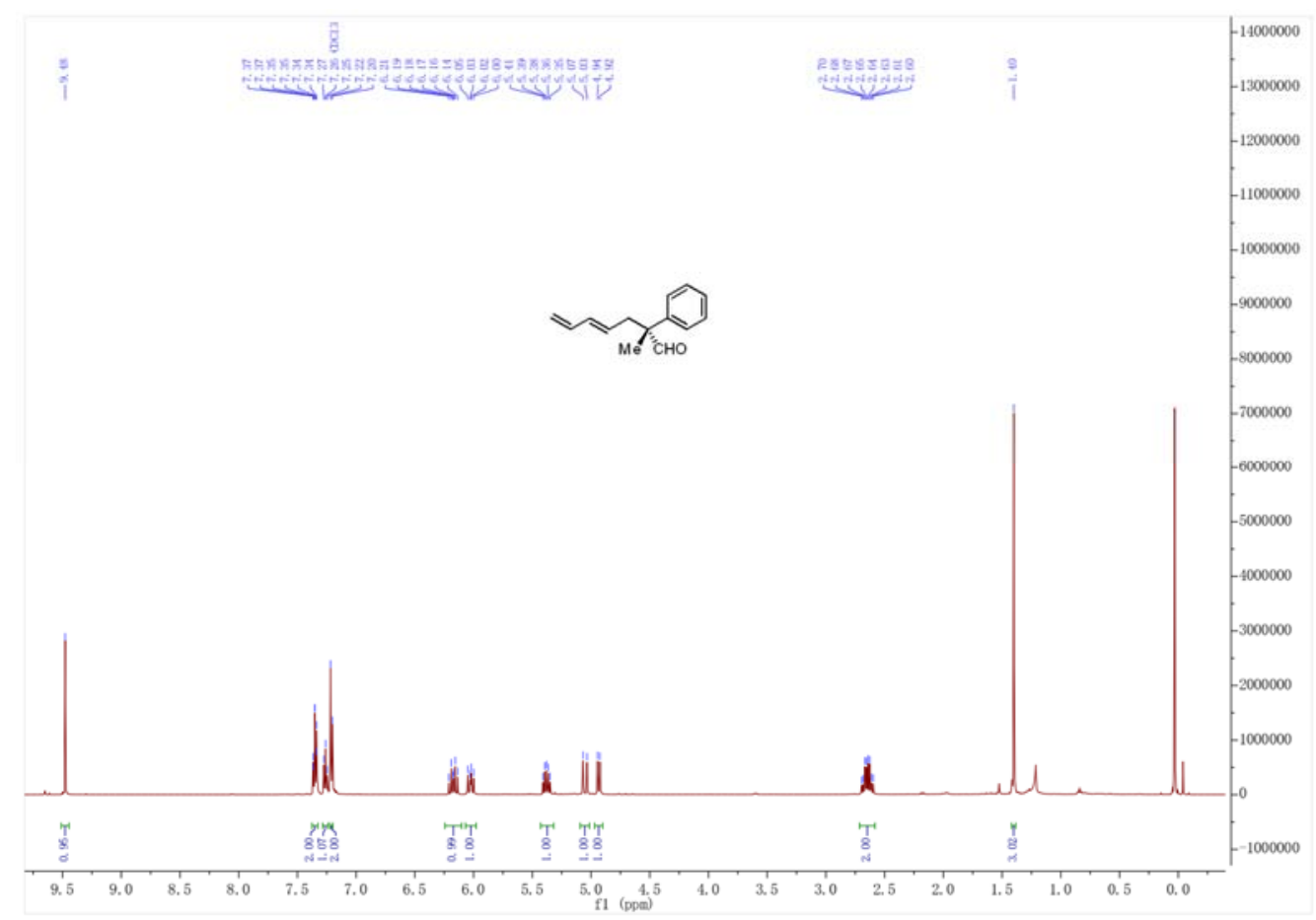

${ }^{13}$ C NMR (126 MHz, Chloroform-d)

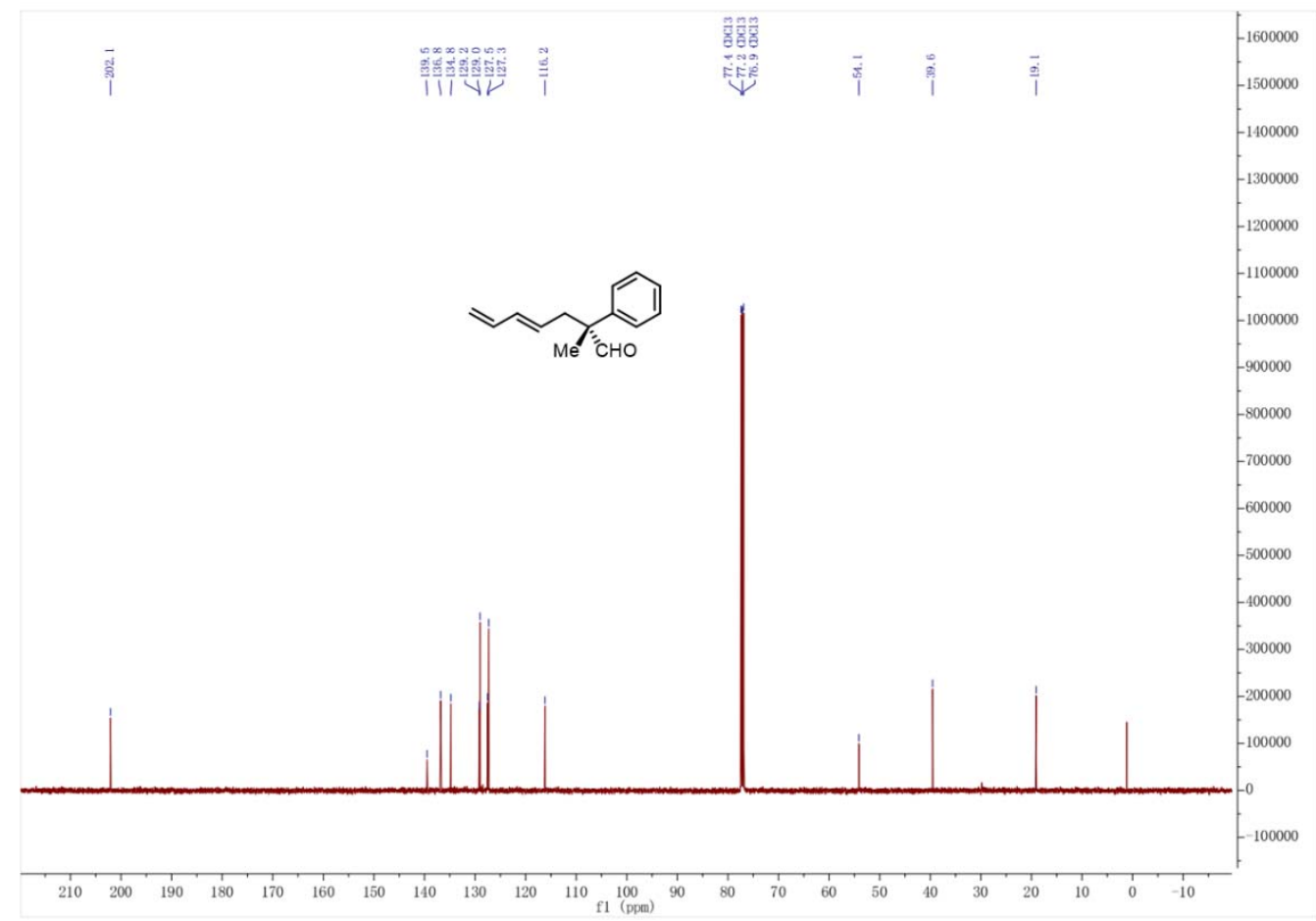


(R,E)-2-methyl-2-(p-tolyl)hepta-4,6-dienal (3b)

${ }^{1} \mathbf{H}$ NMR (500 MHz, Chloroform-d)

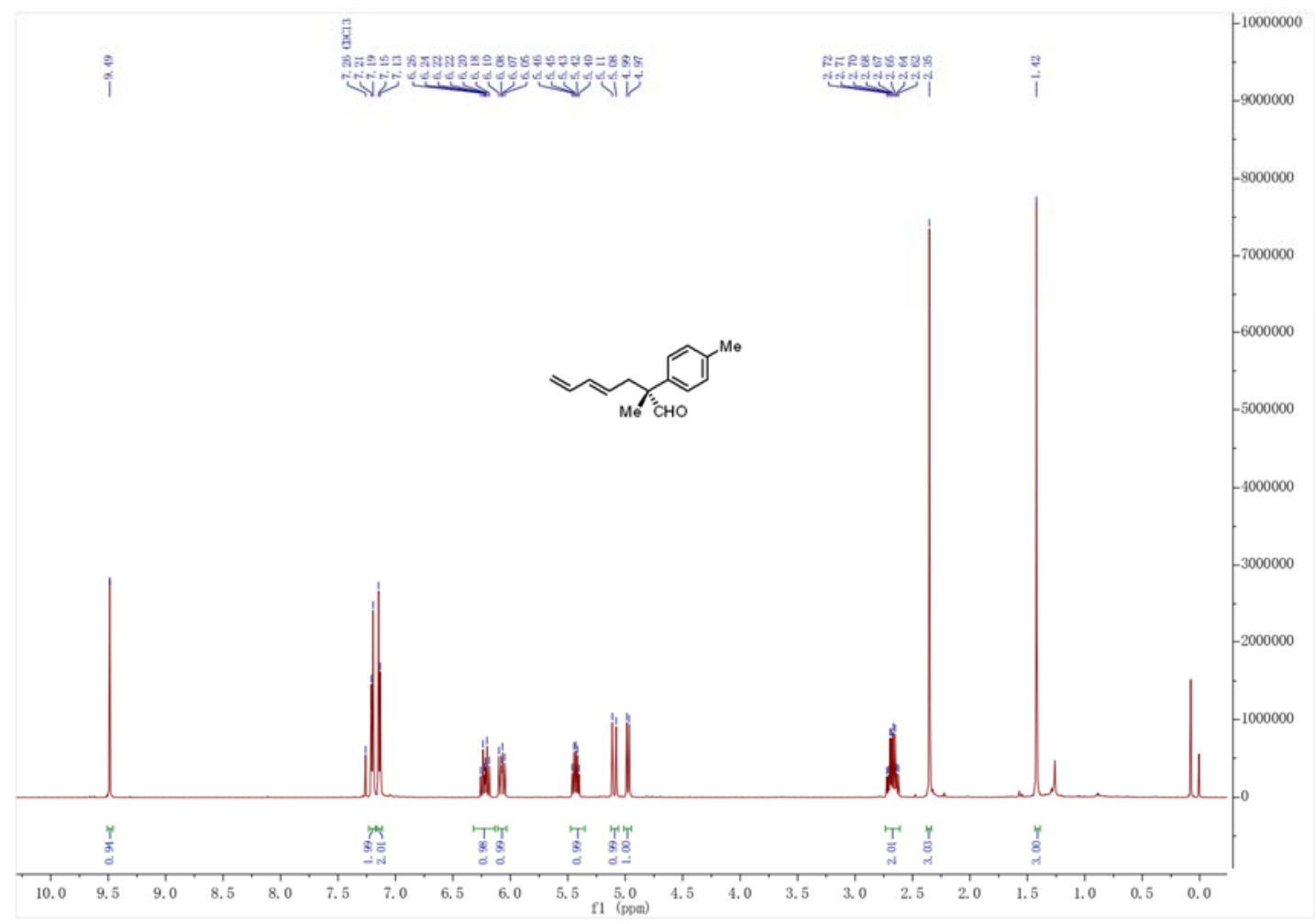

${ }^{13}$ C NMR (126 MHz, Chloroform-d)

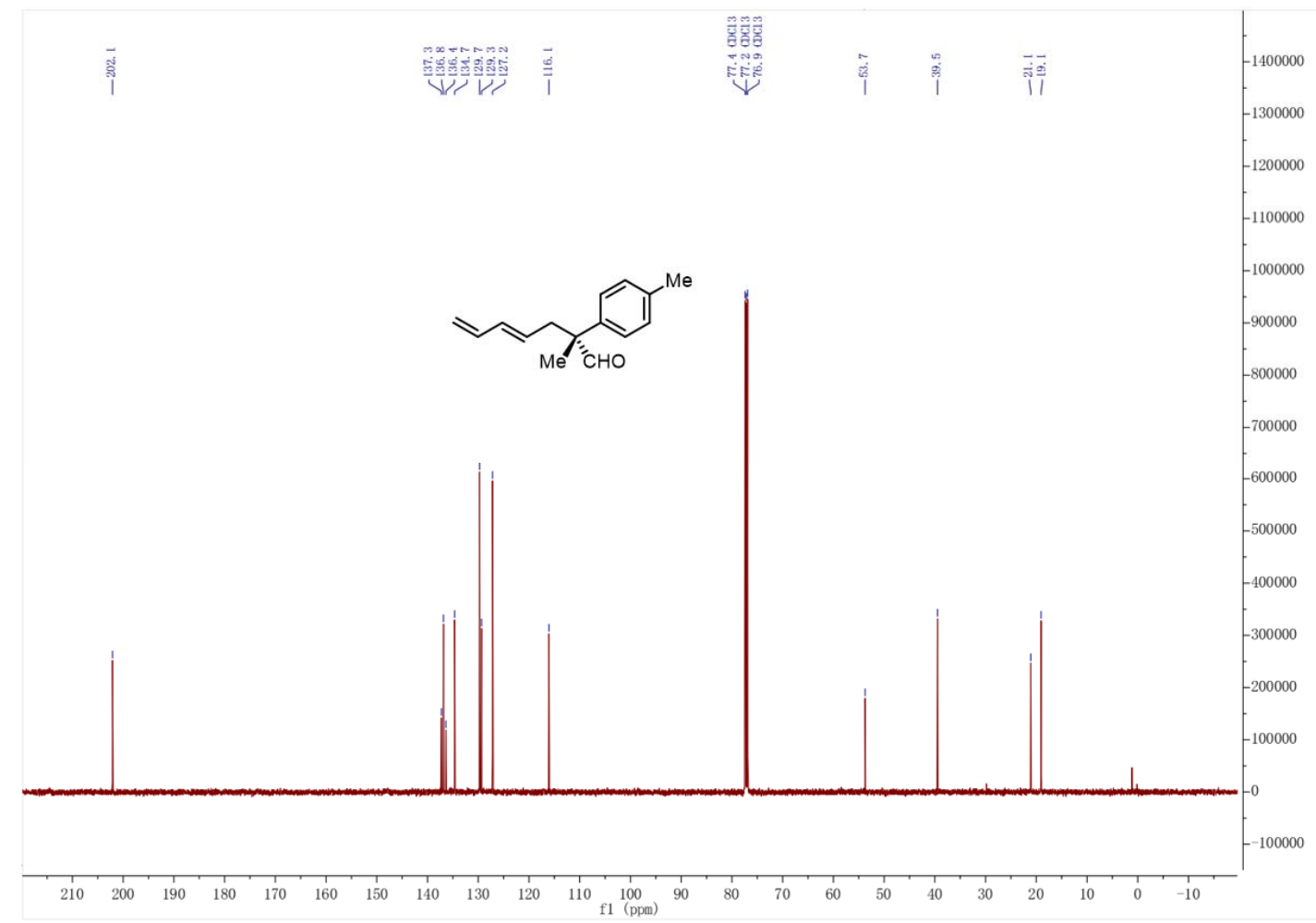


(R,E)-2-(4-methoxyphenyl)-2-methylhepta-4,6-dienal (3c)

${ }^{1}$ H NMR (500 MHz, Chloroform-d)

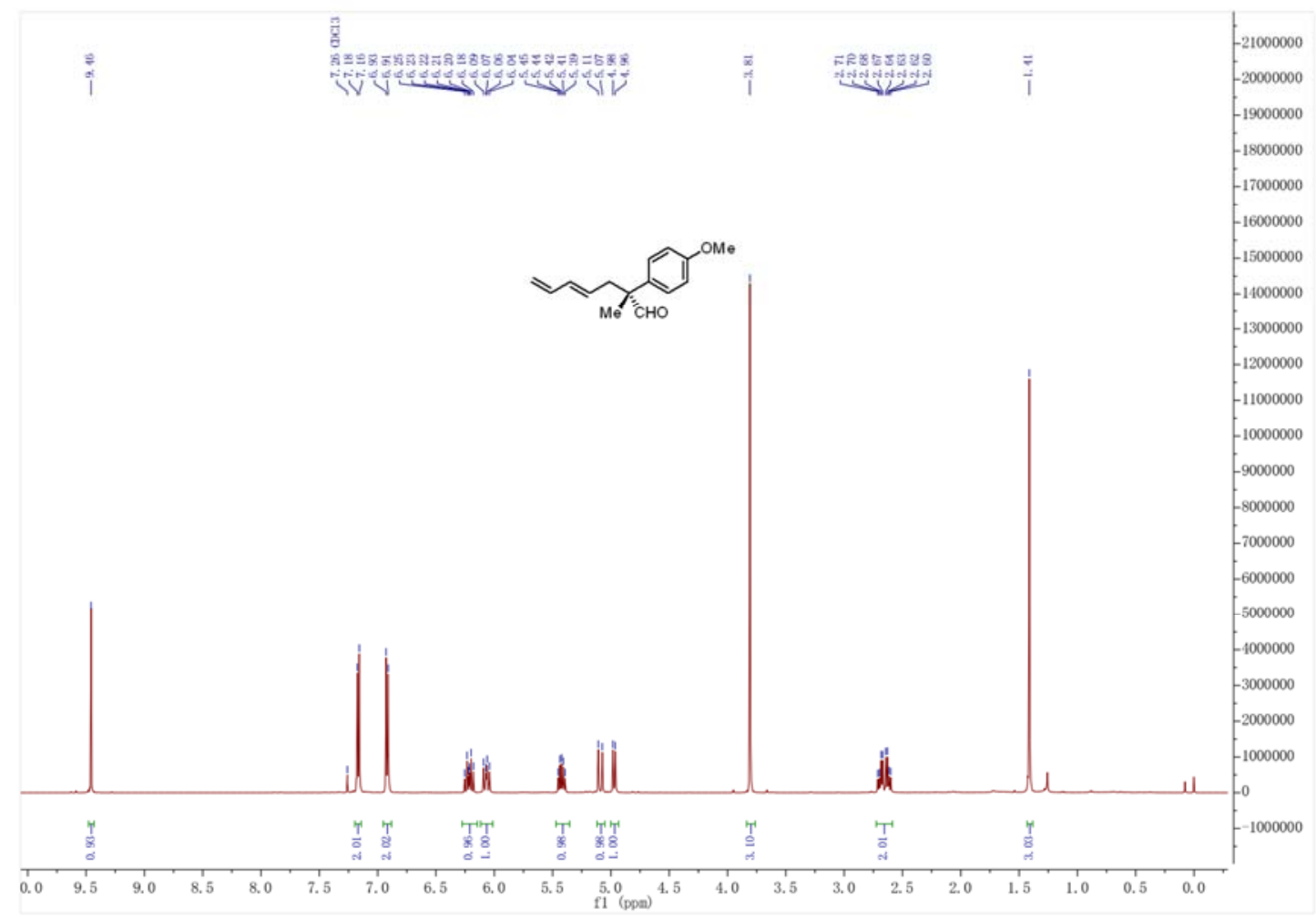

${ }^{13}$ C NMR (126 MHz, Chloroform-d)

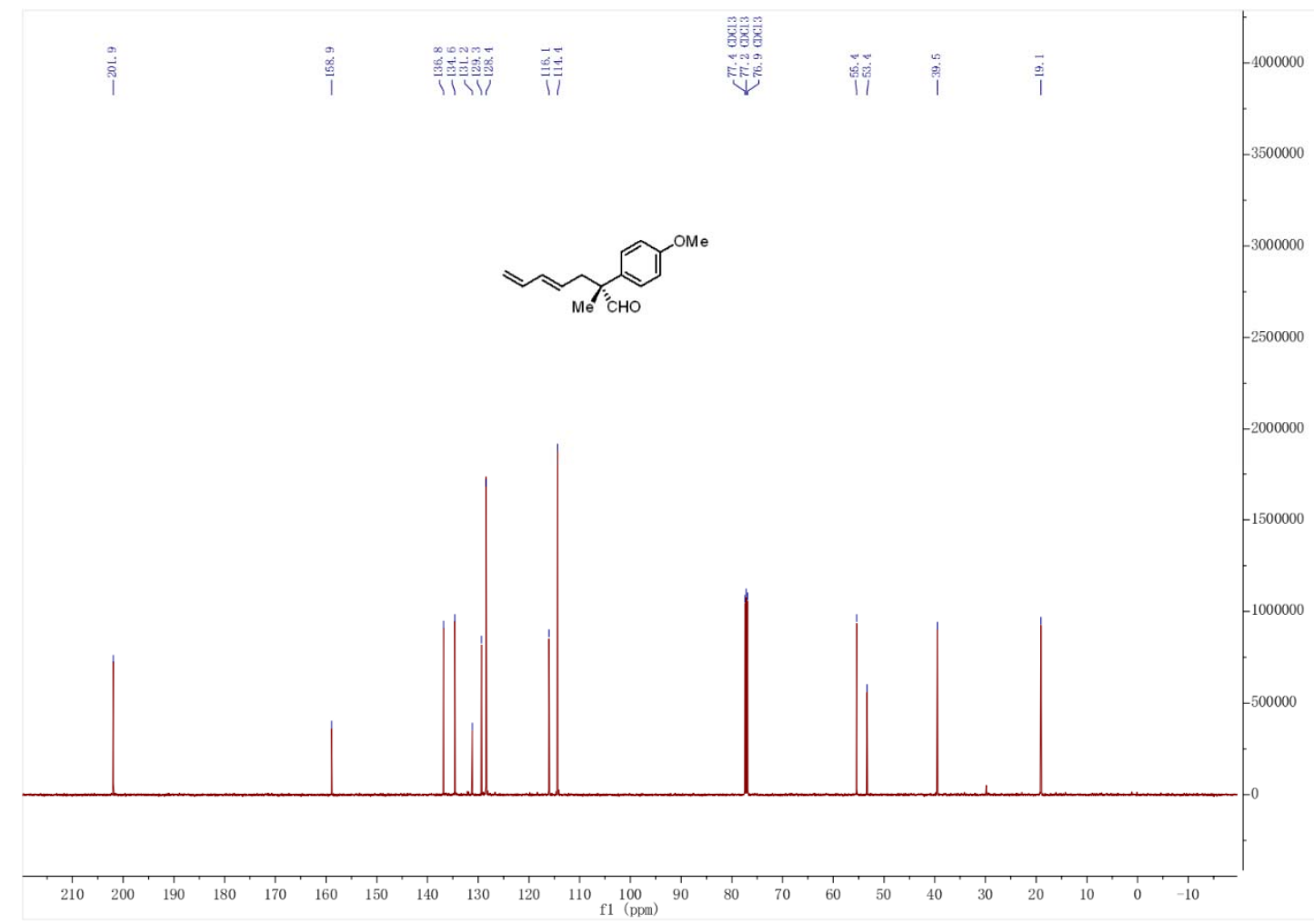


(R,E)-2-(4-fluorophenyl)-2-methylhepta-4,6-dienal (3d)

${ }^{1}$ H NMR (400 MHz, Chloroform-d)

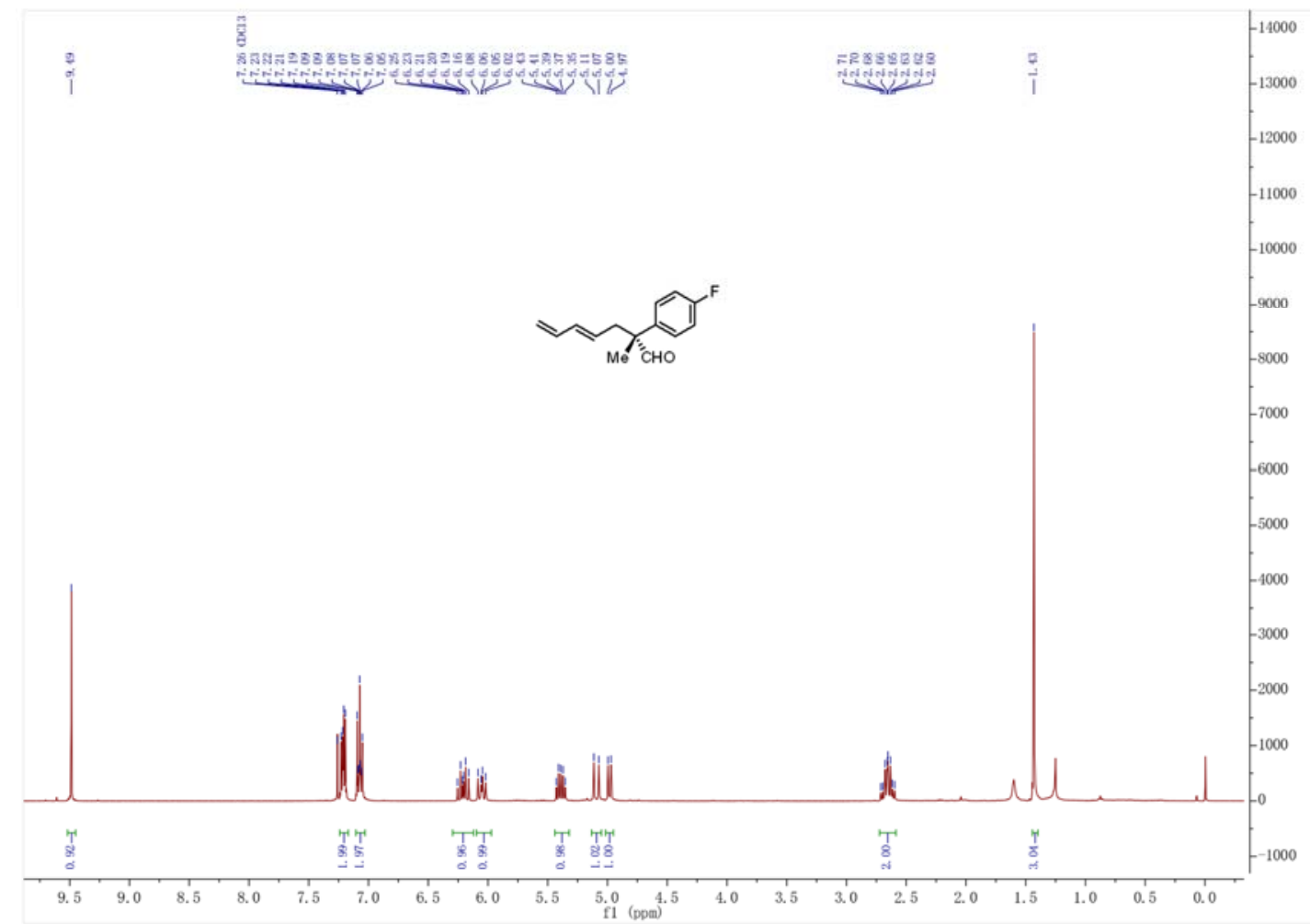

${ }^{13}$ C NMR (126 MHz, Chloroform-d)

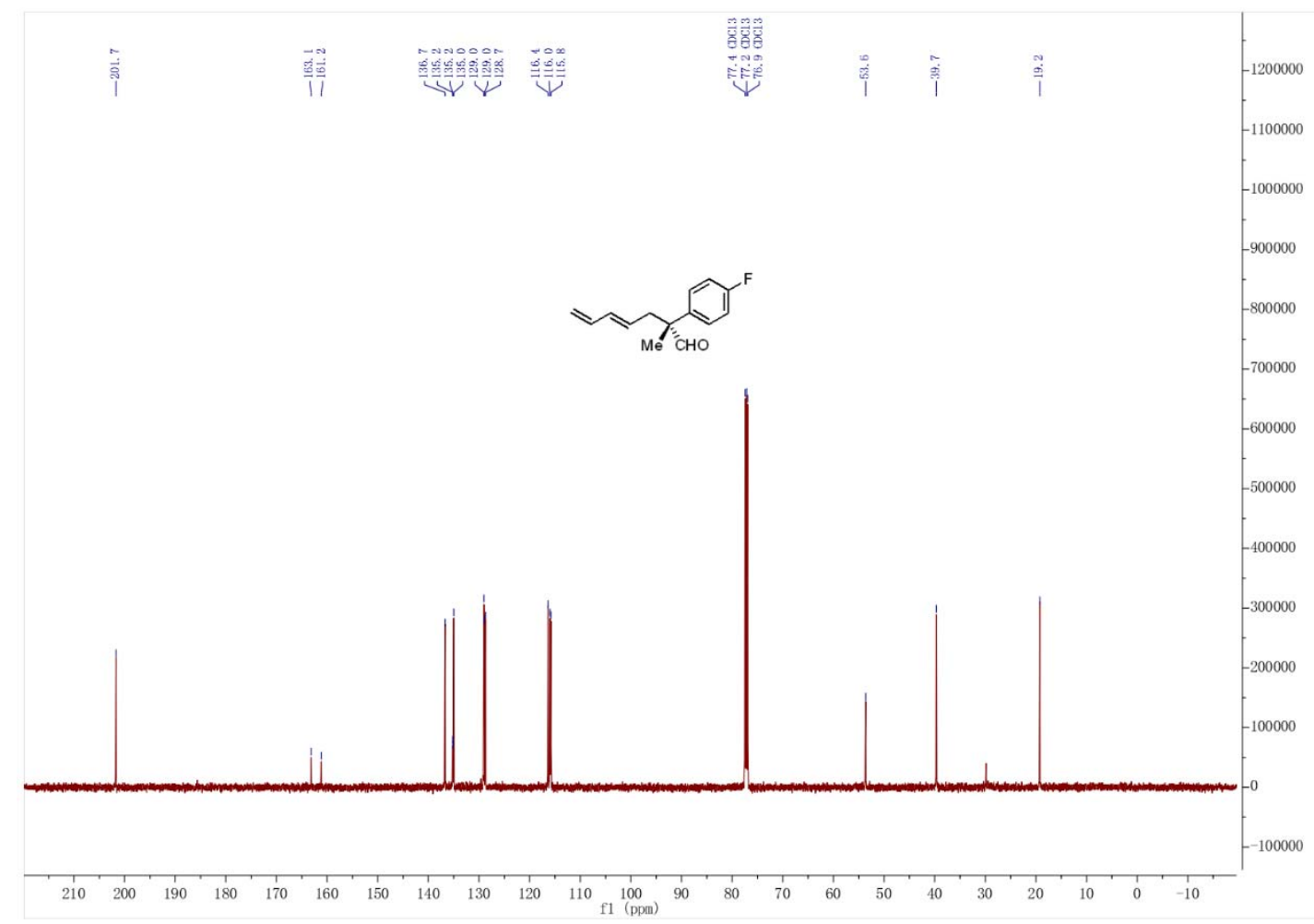


${ }^{19}$ F NMR (376 MHz, Chloroform-d)

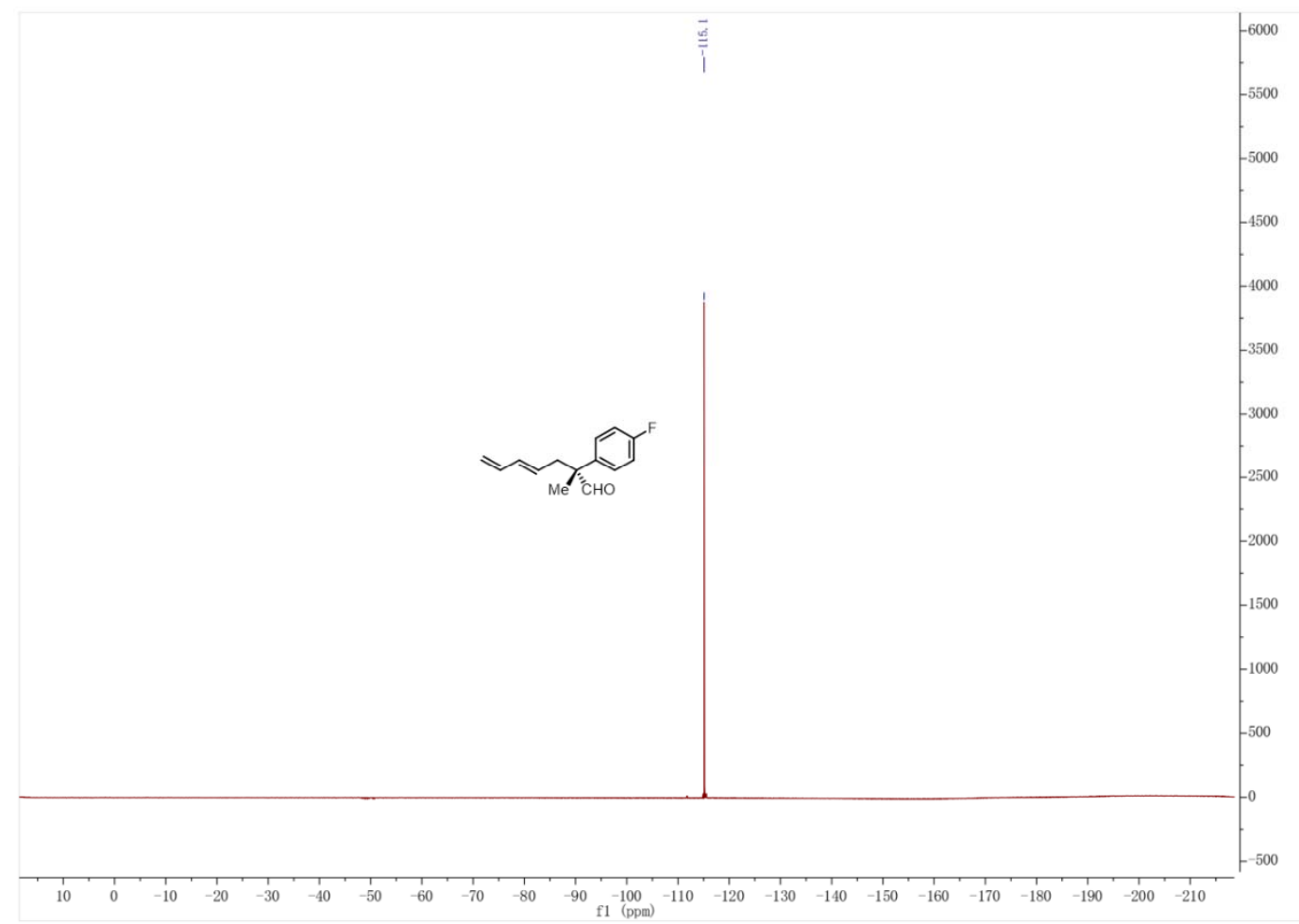

(R,E)-2-(3-chlorophenyl)-2-methylhepta-4,6-dienal (3e)

${ }^{1}$ H NMR (500 MHz, Chloroform-d)

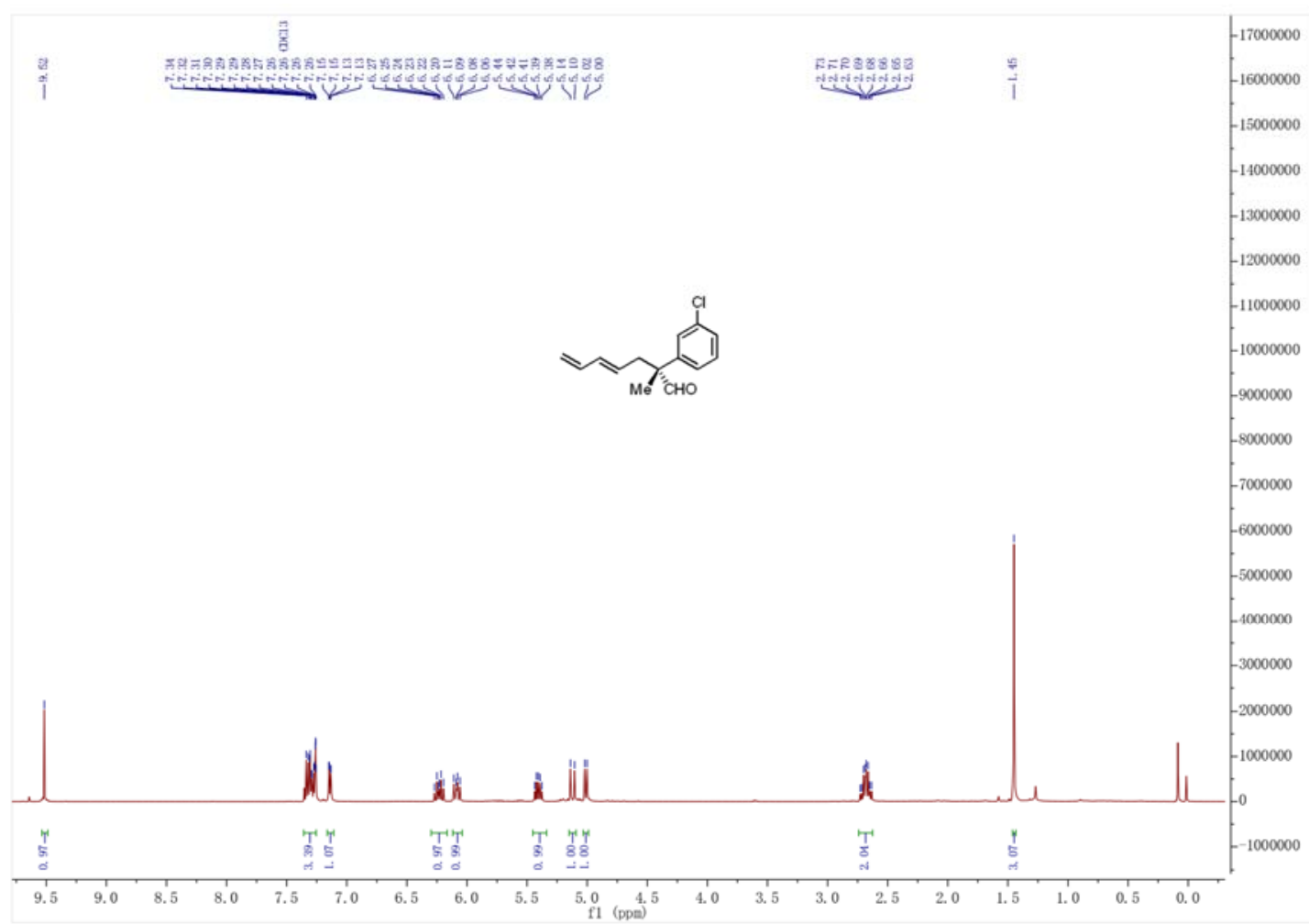


${ }^{13}$ C NMR (126 MHz, Chloroform-d)

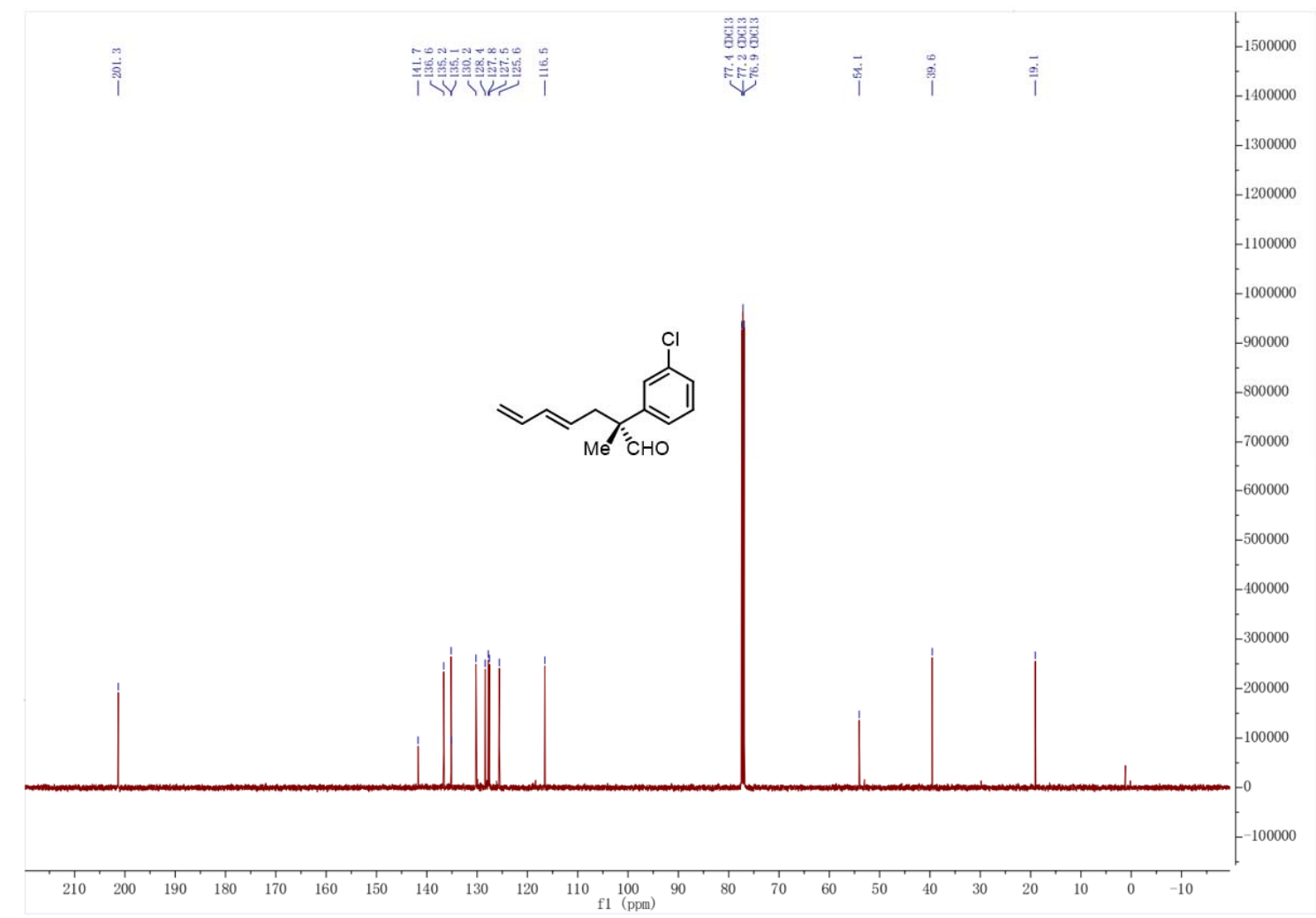

(R,E)-2-methyl-2-(m-tolyl)hepta-4,6-dienal (3f)

${ }^{1}$ H NMR (400 MHz, Chloroform-d)

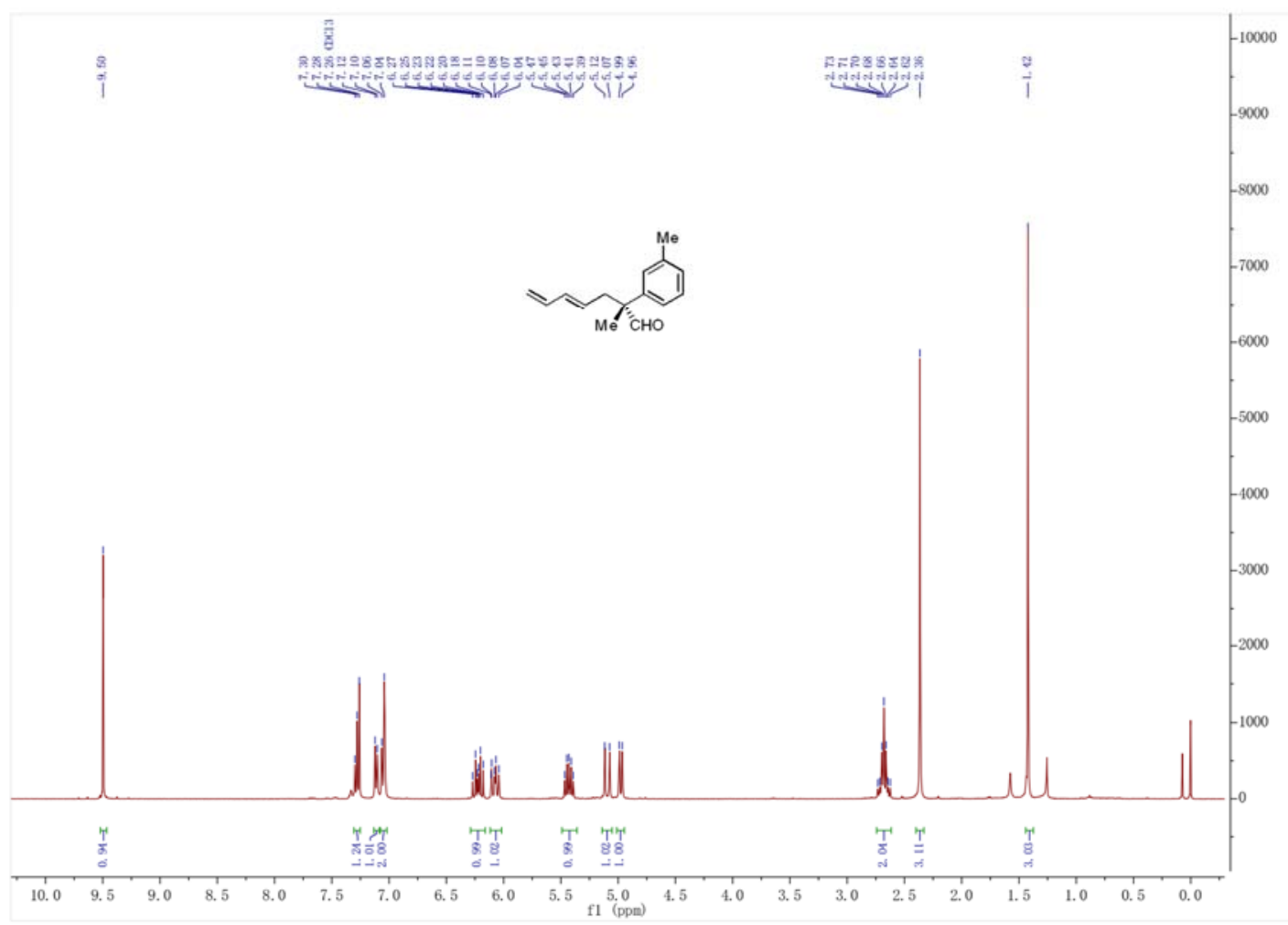


${ }^{13}$ C NMR (101 MHz, Chloroform-d)

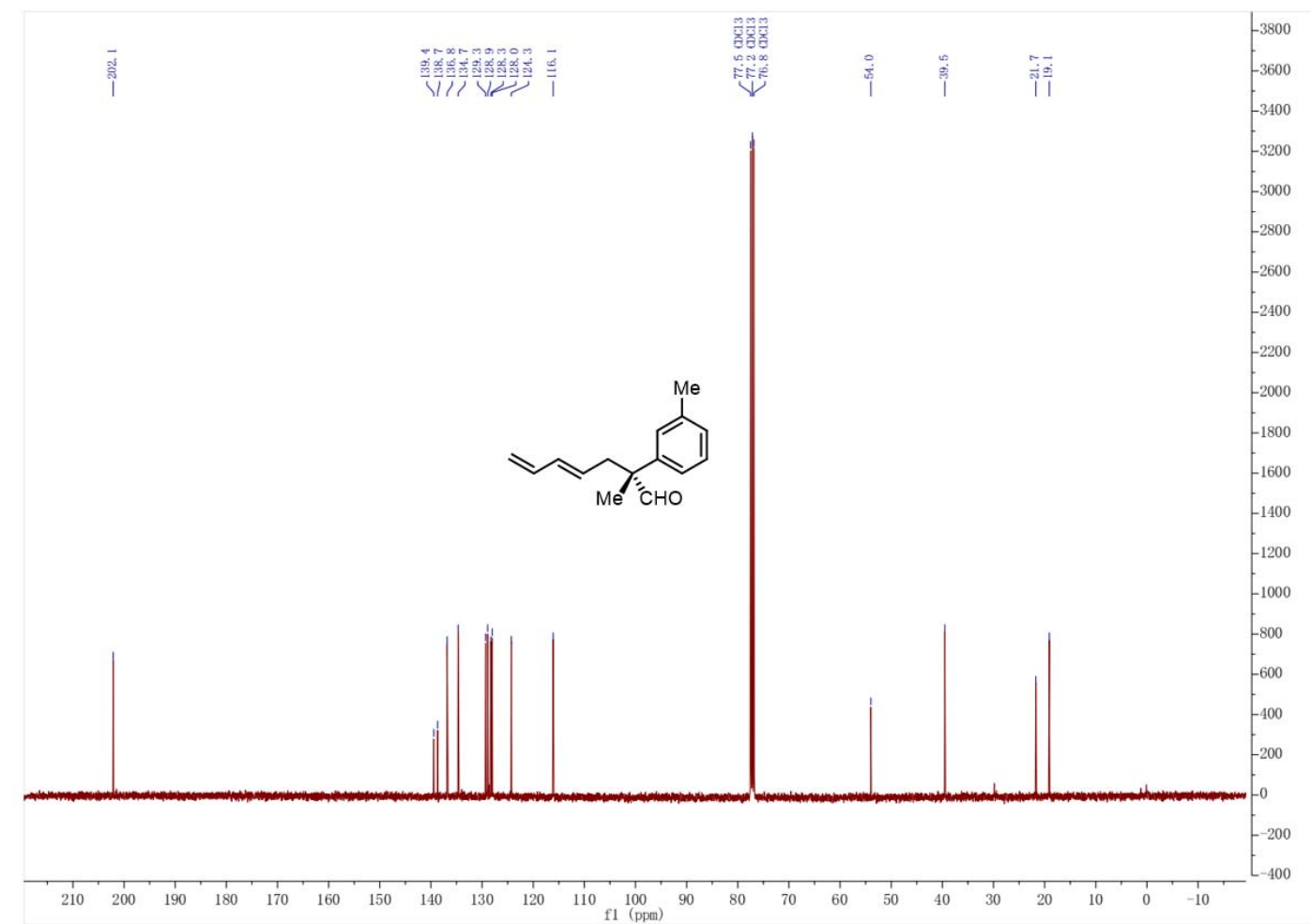

(R,E)-2-(2-methoxyphenyl)-2-methylhepta-4,6-dienal (3g)

${ }^{1}$ H NMR (500 MHz, Chloroform- $d$ )

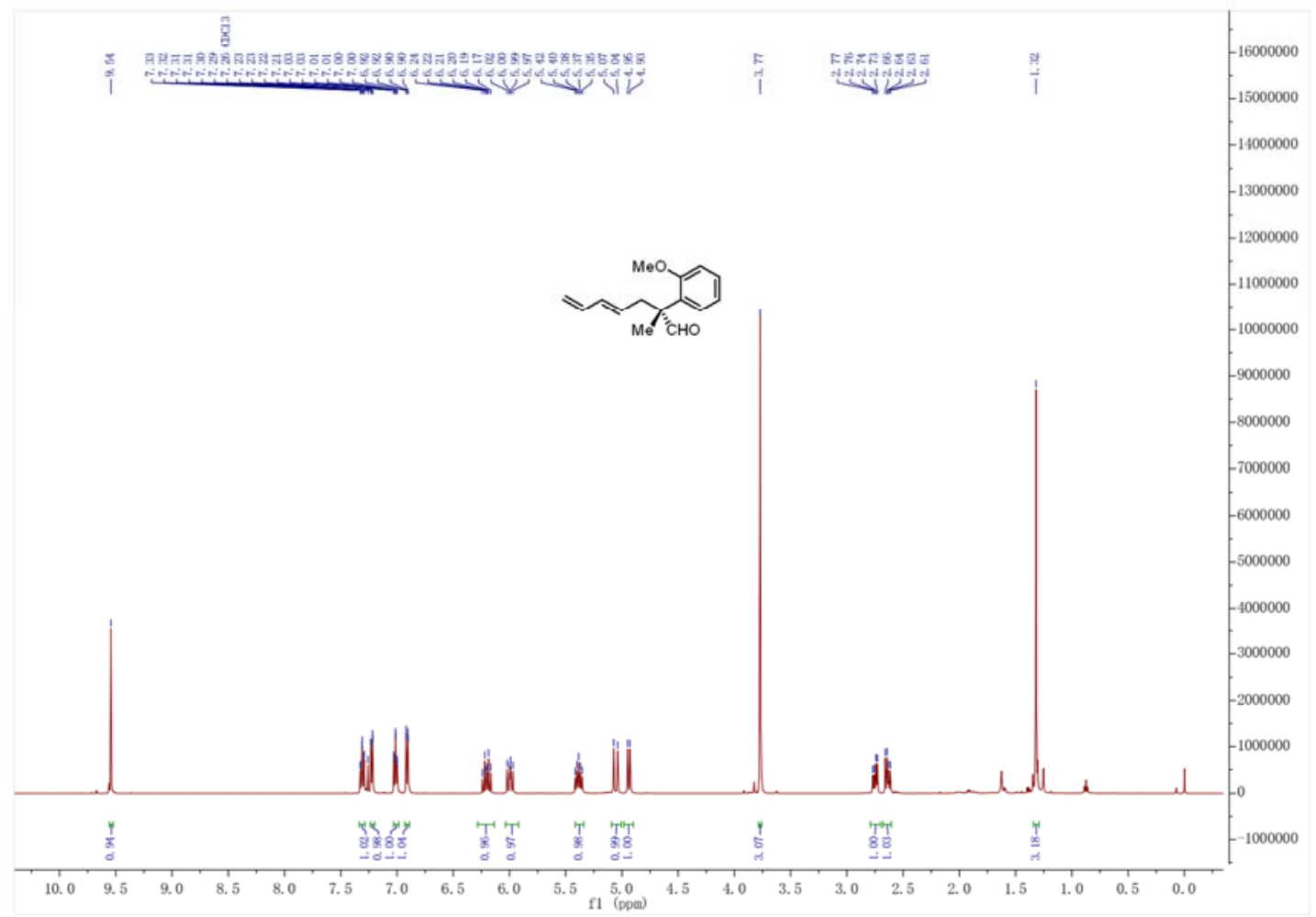


${ }^{13}$ C NMR (126 MHz, Chloroform-d)

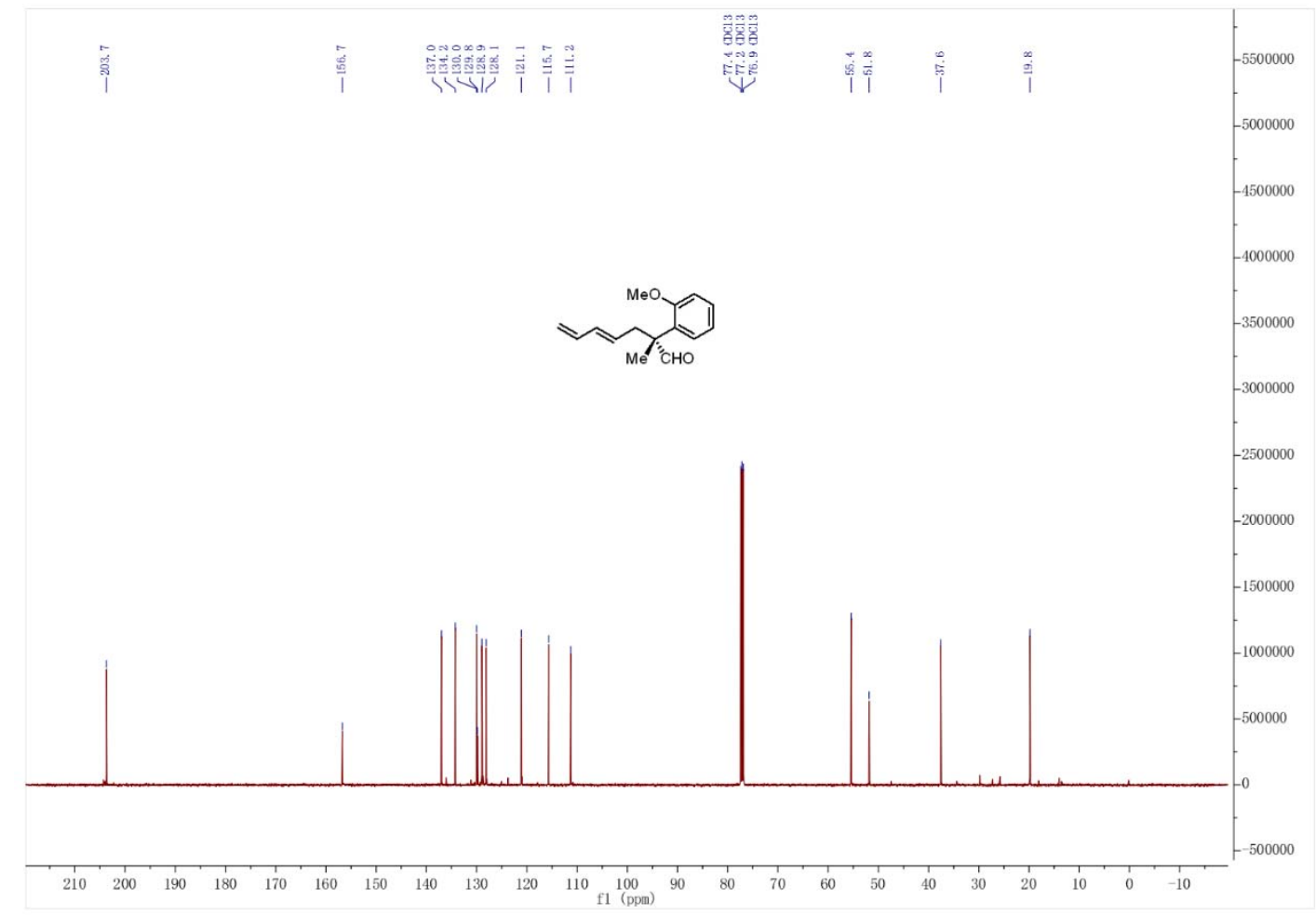

(R,E)-2-(2-fluorophenyl)-2-methylhepta-4,6-dienal (3h)

${ }^{1}$ H NMR (400 MHz, Chloroform-d)

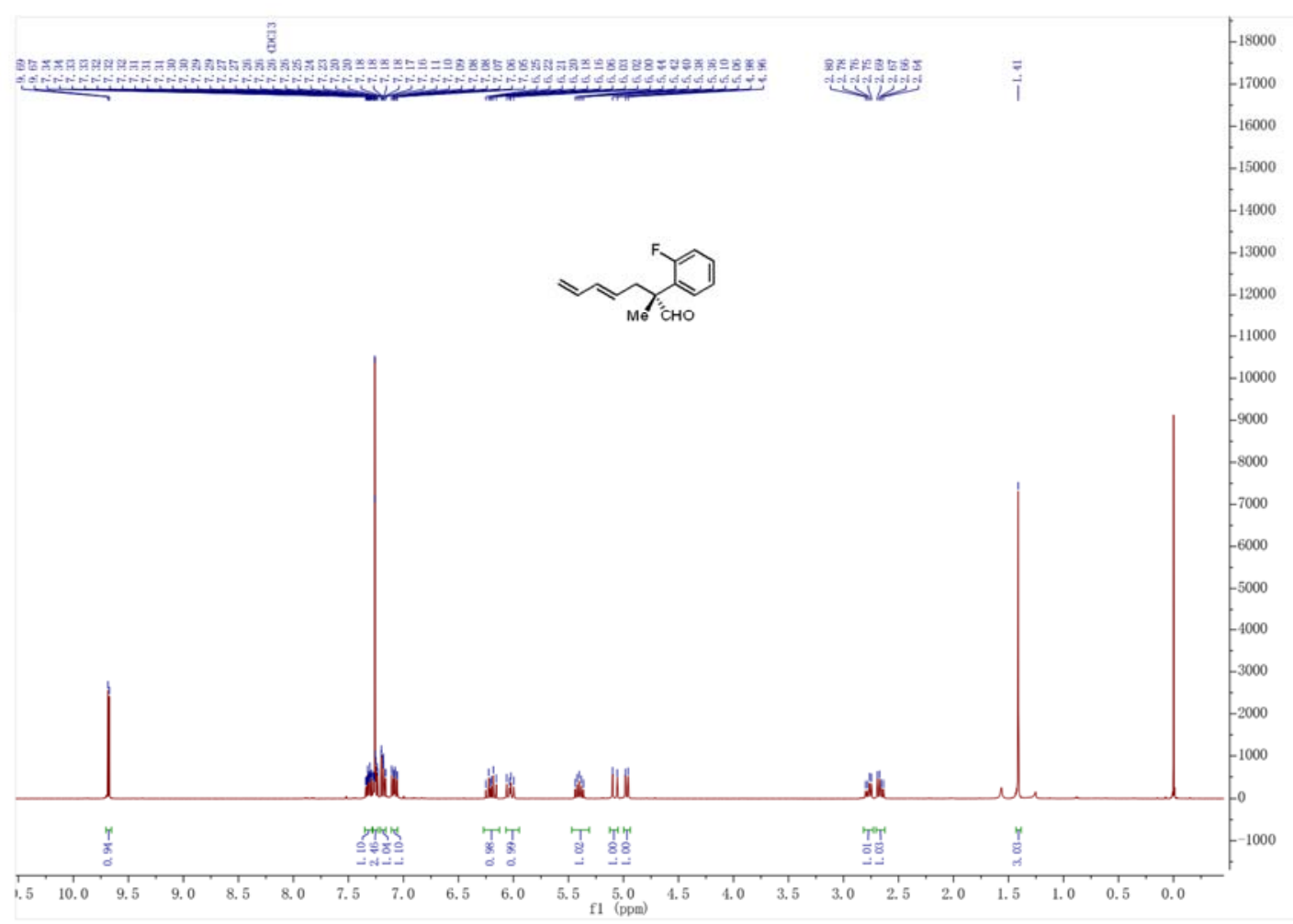


${ }^{13}$ C NMR (126 MHz, Chloroform-d)

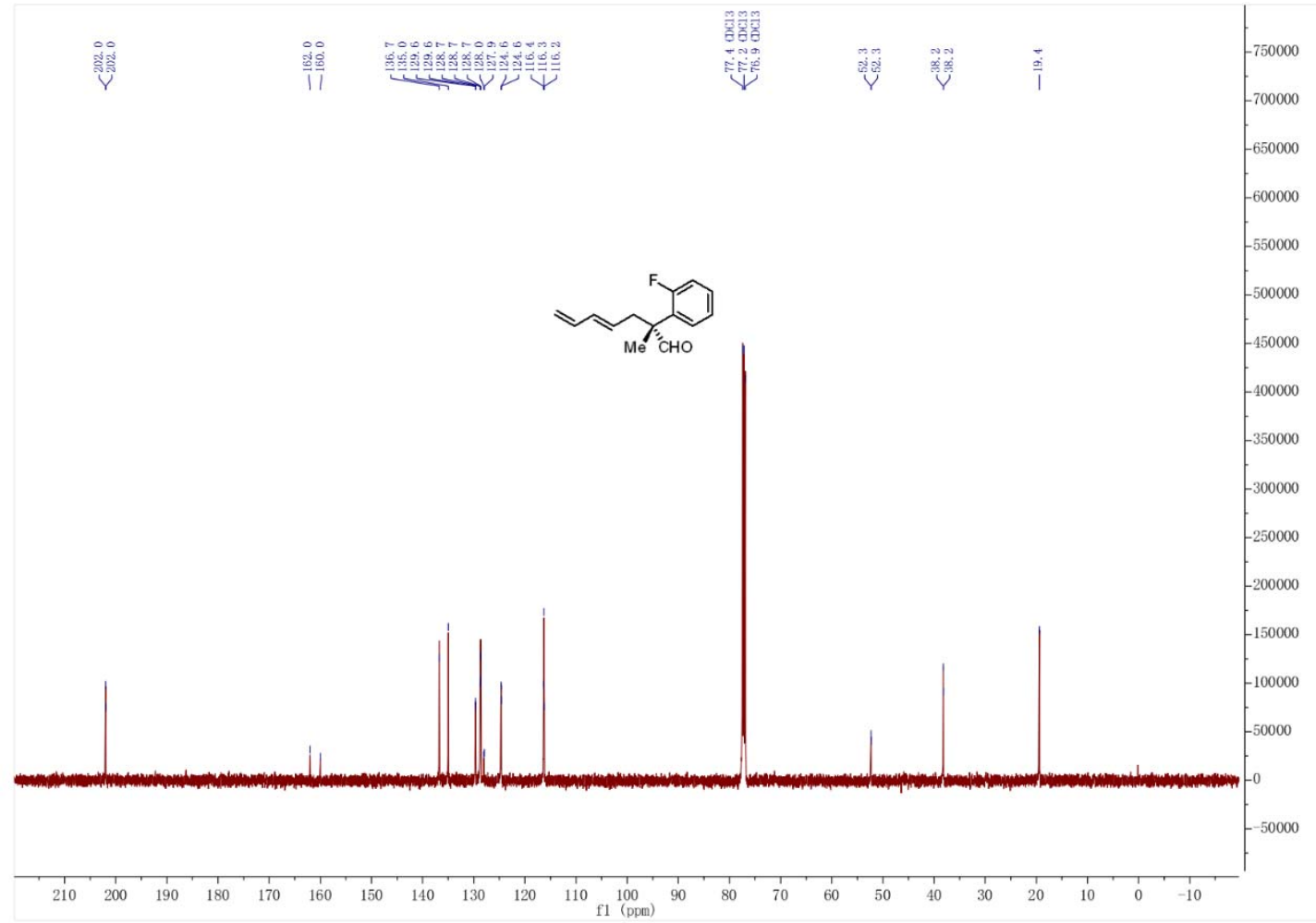

${ }^{19}$ F NMR (471 MHz, Chloroform-d)

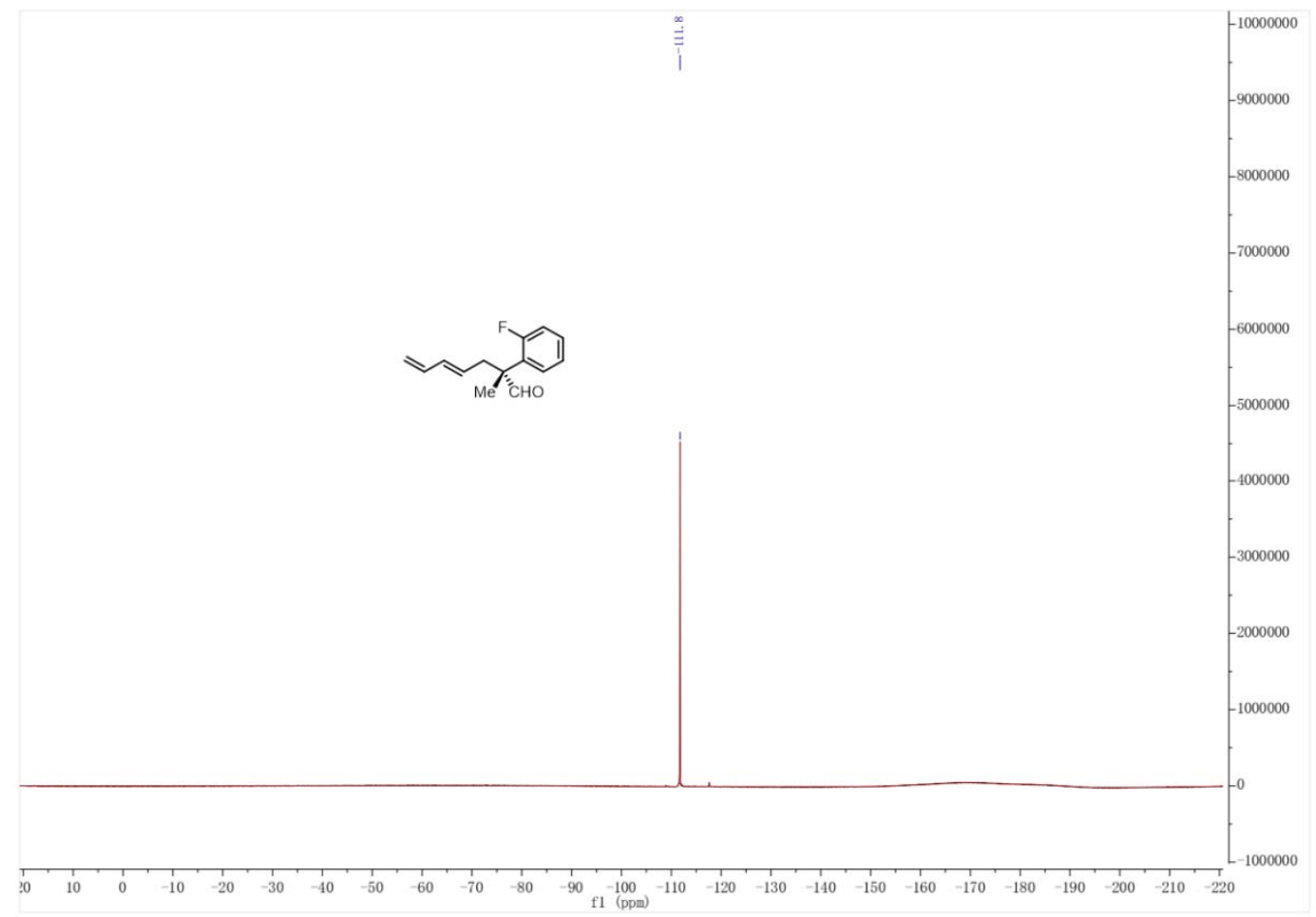


(R,E)-2-(9H-fluoren-2-yl)-2-methylhepta-4,6-dienal (3i)

${ }^{1}$ H NMR (500 MHz, Chloroform-d)

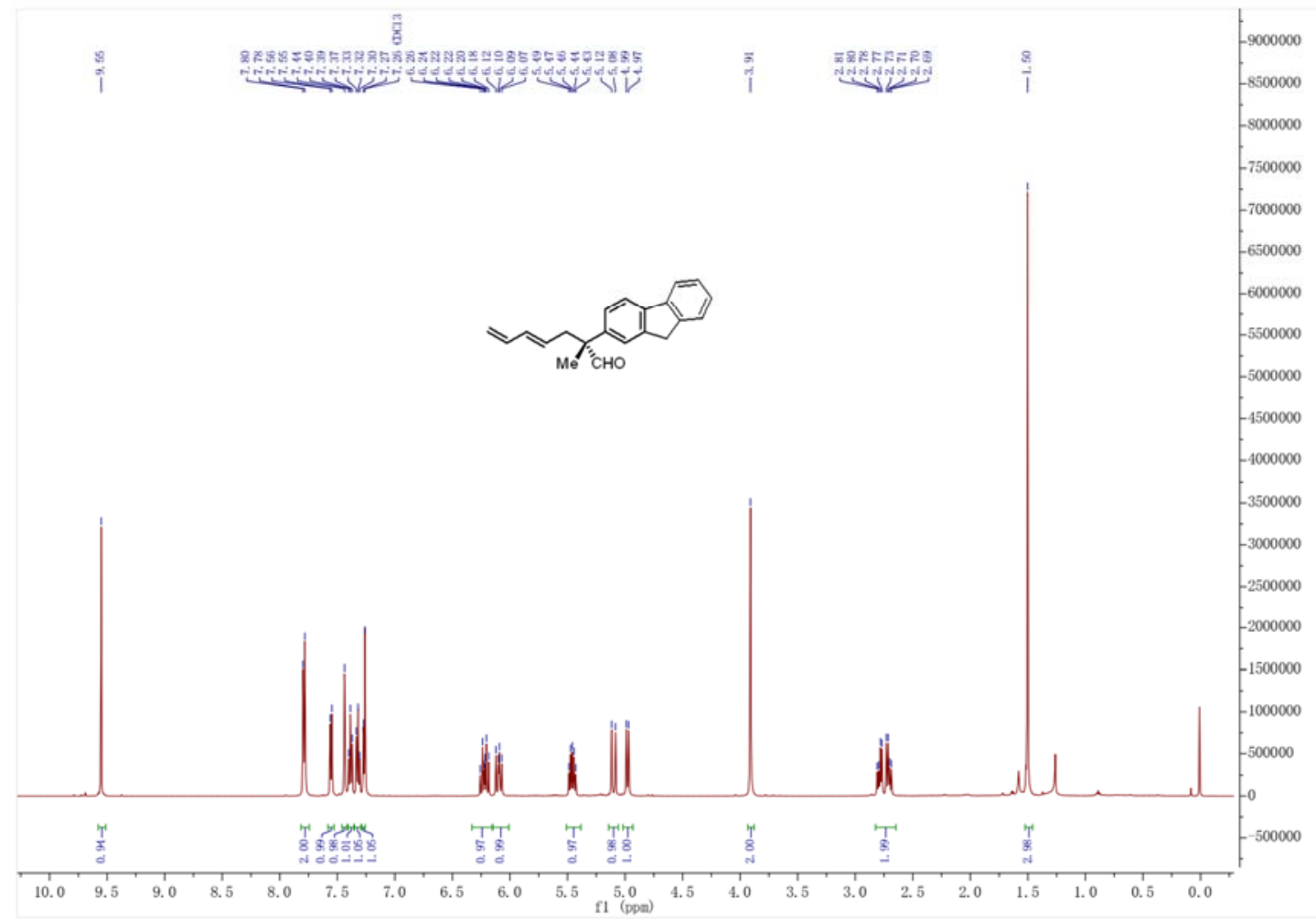

${ }^{13}$ C NMR (126 MHz, Chloroform-d)

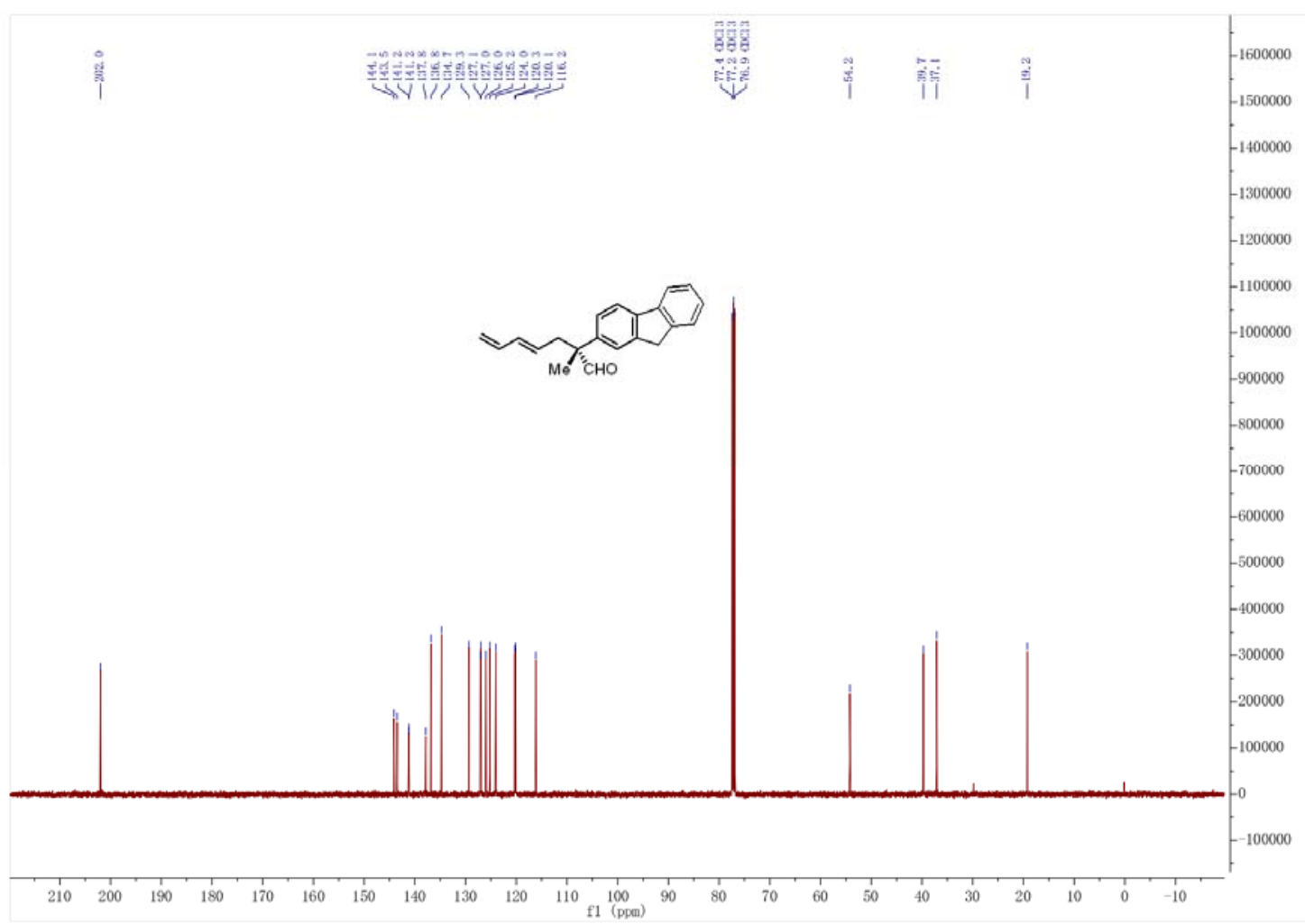


(R,E)-2-methyl-2-(naphthalen-2-yl)hepta-4,6-dienal (3j)

${ }^{1}$ H NMR (400 MHz, Chloroform-d)

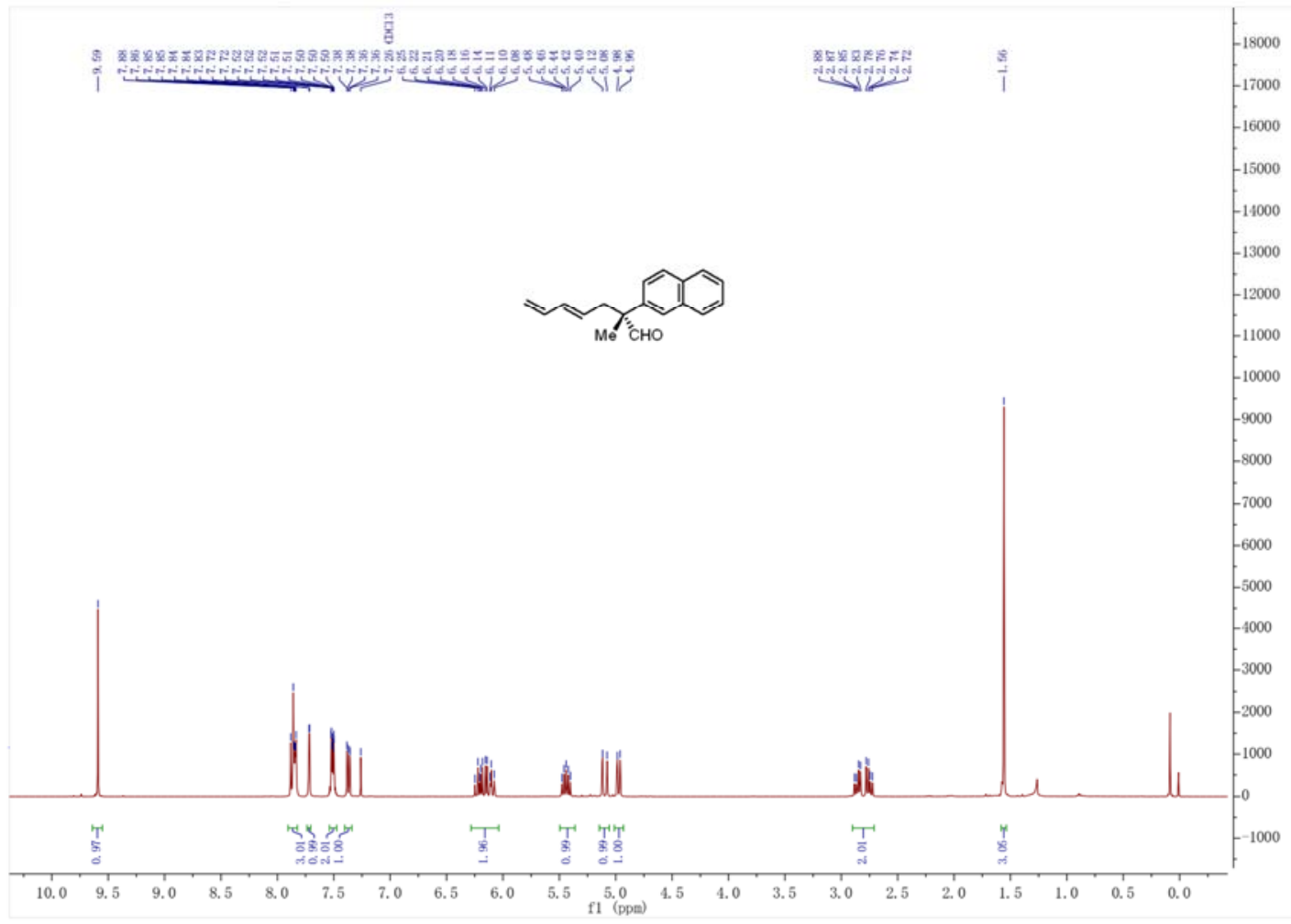

${ }^{13}$ C NMR (126 MHz, Chloroform-d)

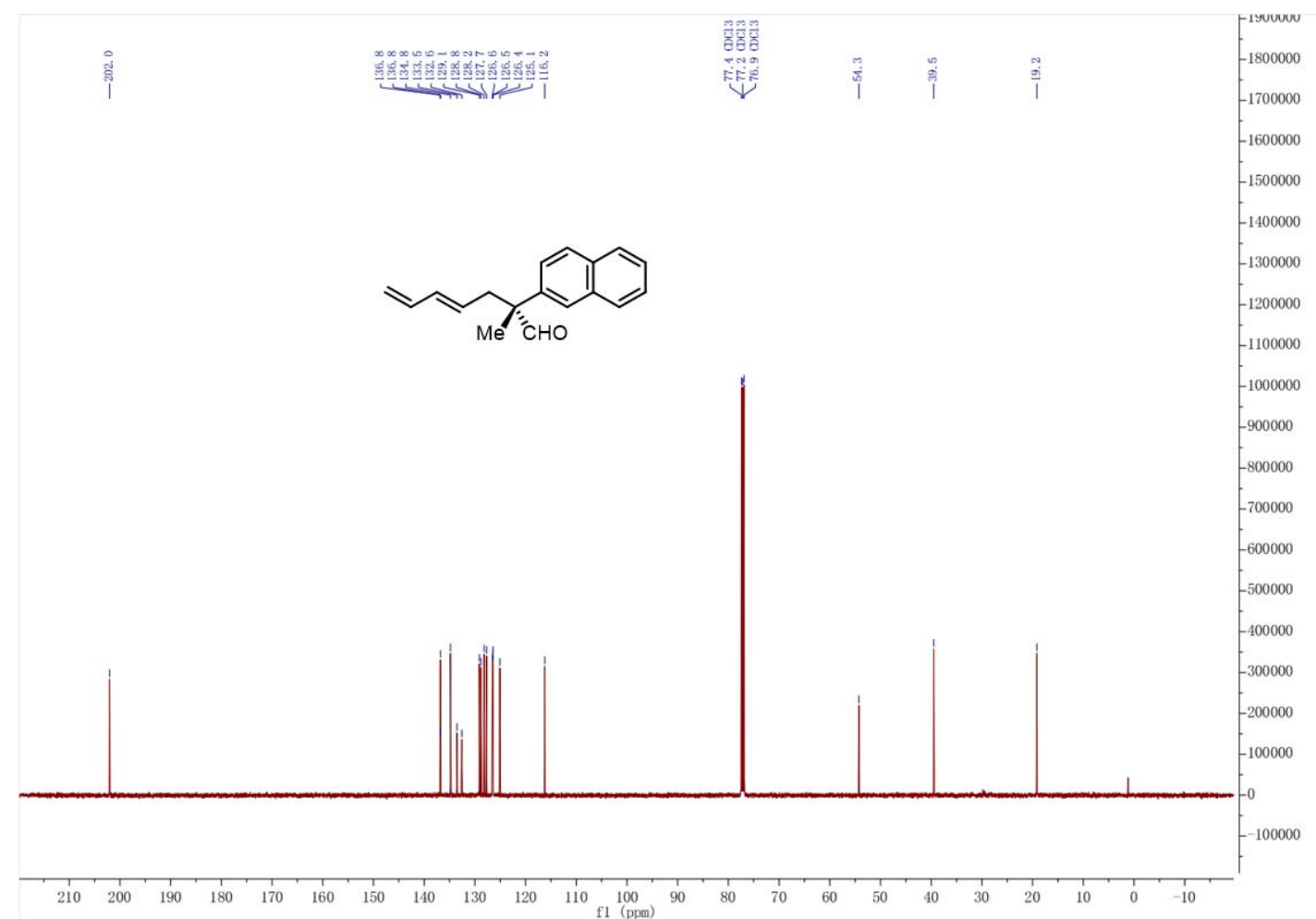


(R,E)-2-ethyl-2-phenylhepta-4,6-dienal (3k)

${ }^{1}$ H NMR (500 MHz, Chloroform-d)

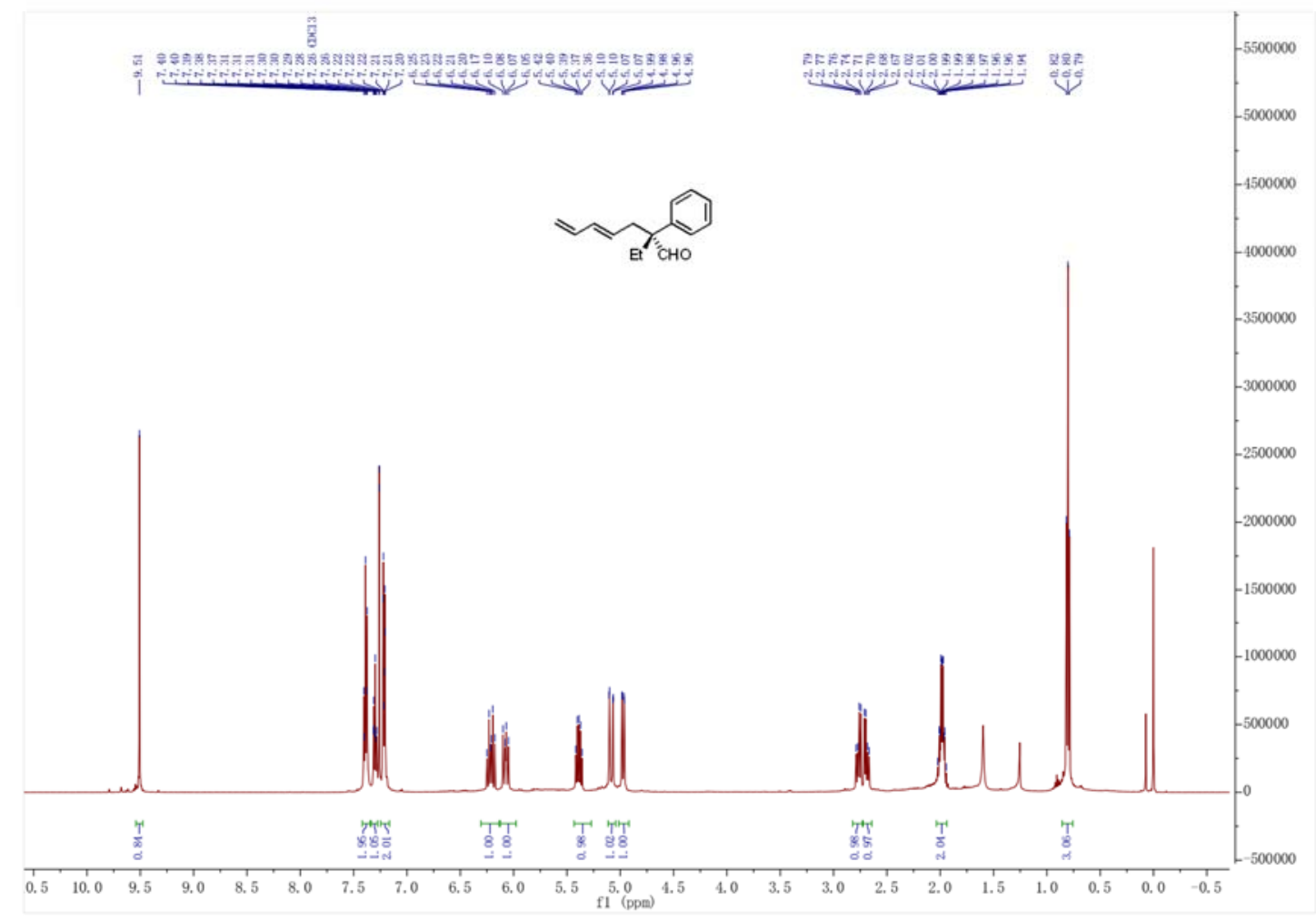

${ }^{13}$ C NMR (126 MHz, Chloroform-d)

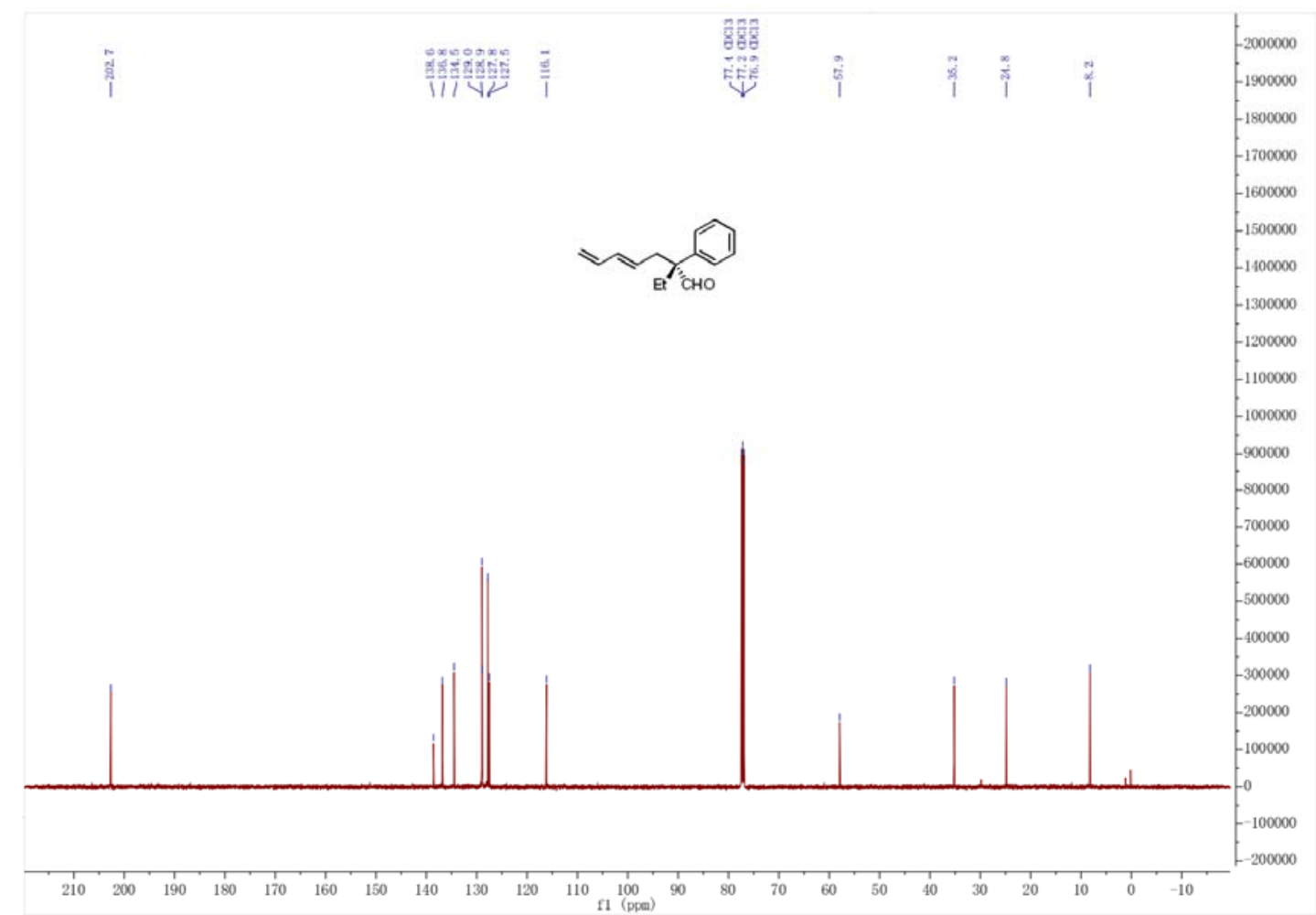


(S,E)-2-cyclopropyl-2-phenylhepta-4,6-dienal (3I)

${ }^{1} \mathbf{H}$ NMR (500 MHz, Chloroform-d)

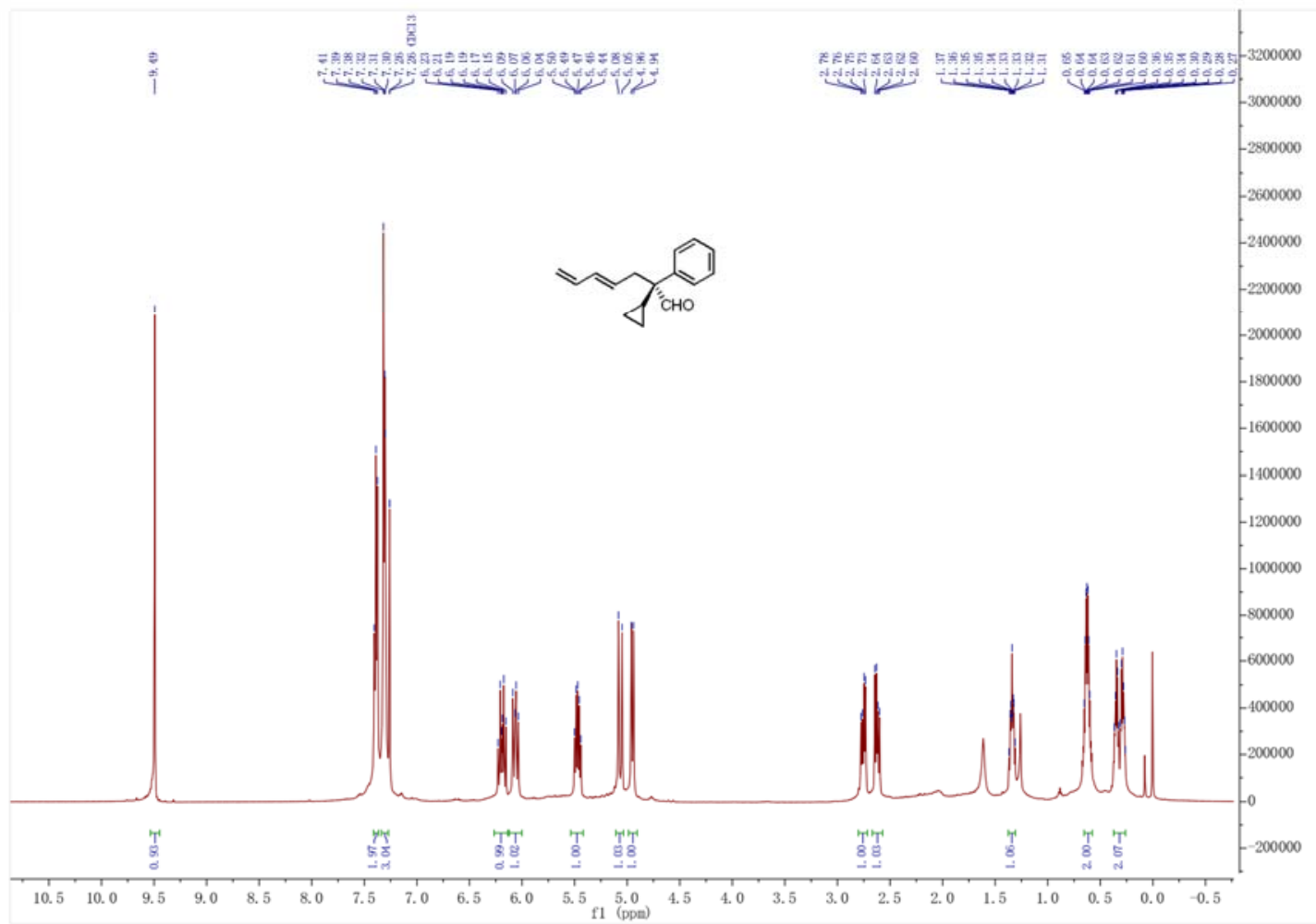

${ }^{13}$ C NMR (126 MHz, Chloroform-d)

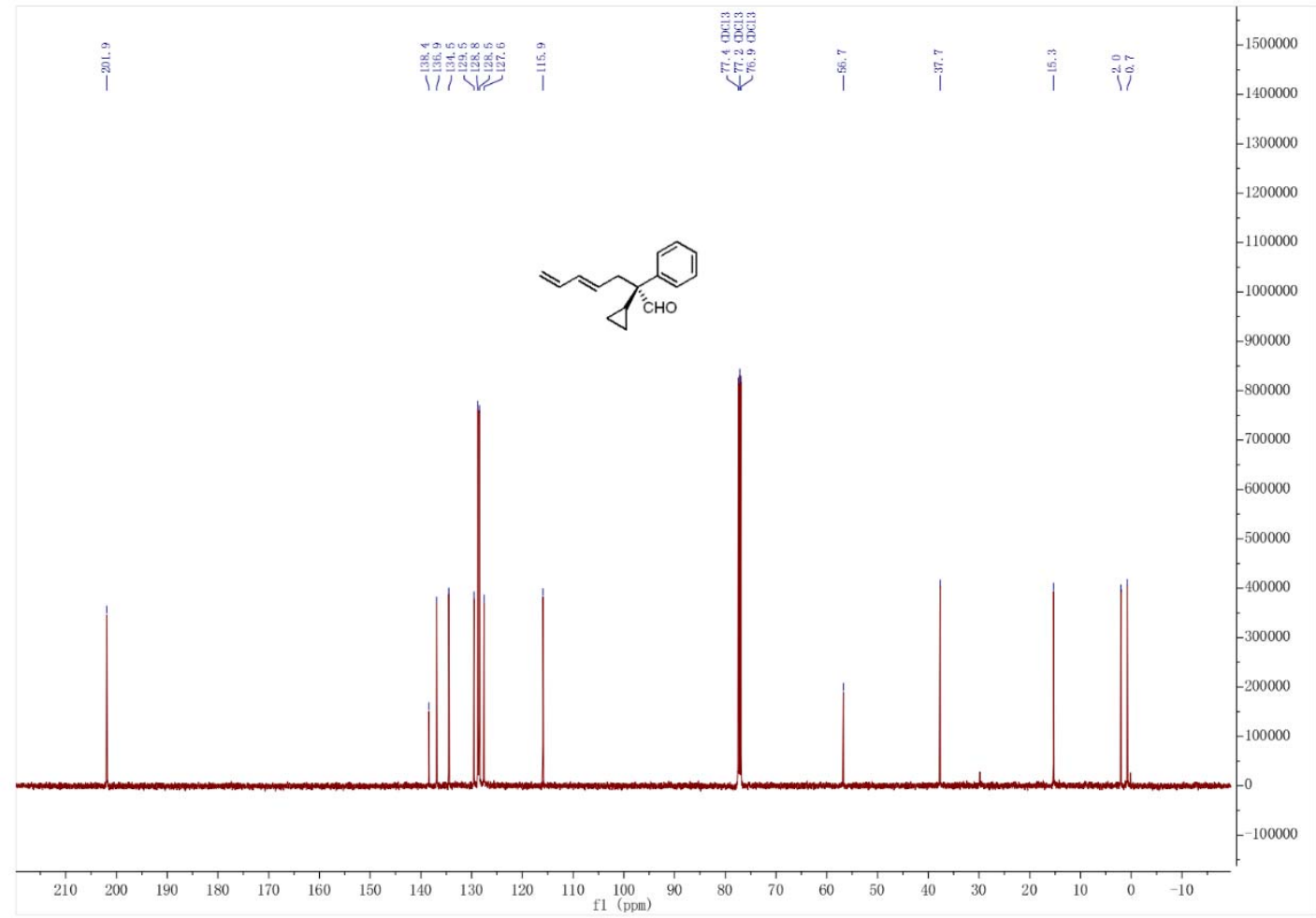


(R,E)-1-(penta-2,4-dien-1-yl)-2,3-dihydro-1H-indene-1-carbaldehyde (3m)

${ }^{1}$ H NMR (400 MHz, Chloroform-d)

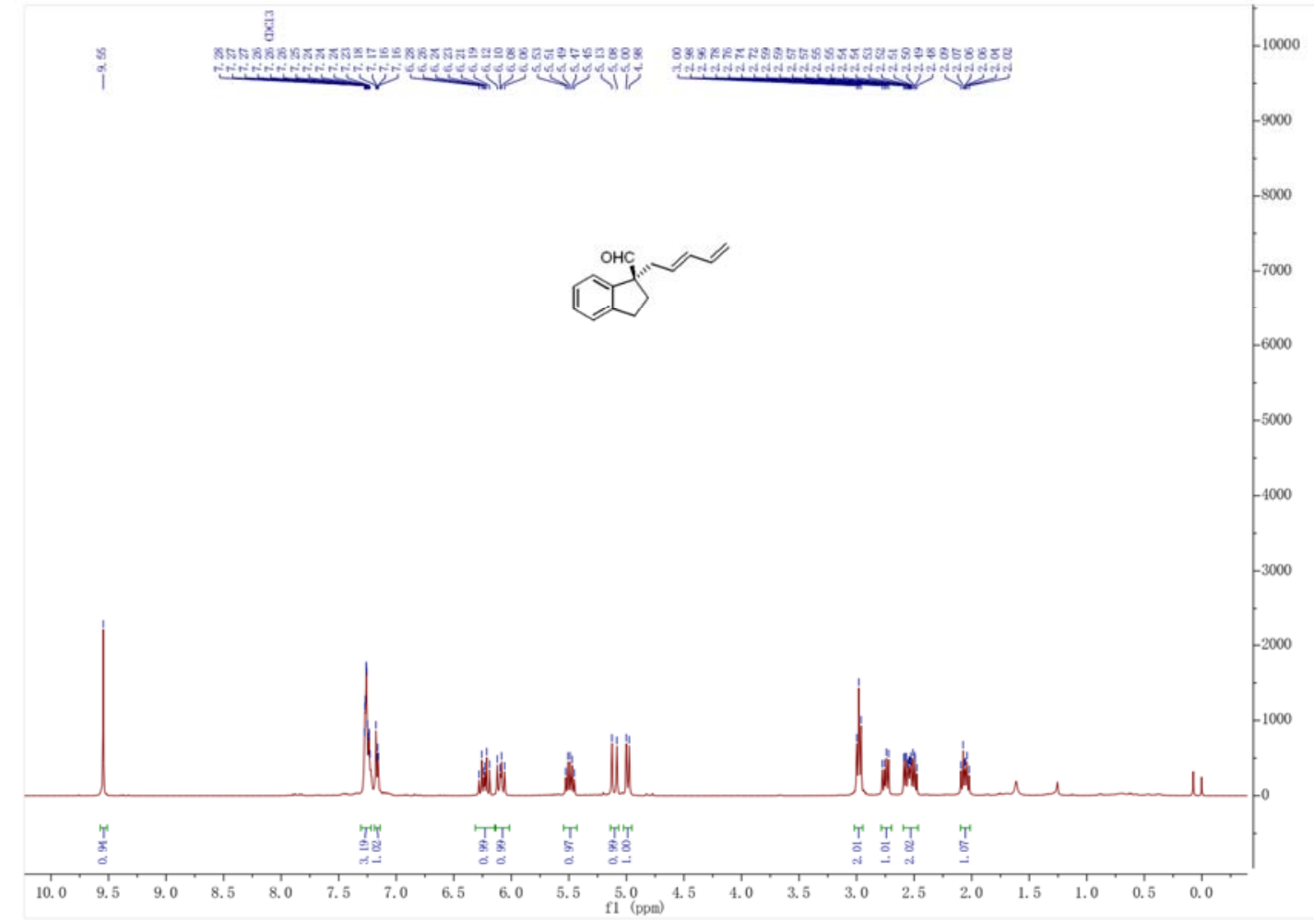

${ }^{13}$ C NMR (126 MHz, Chloroform-d)

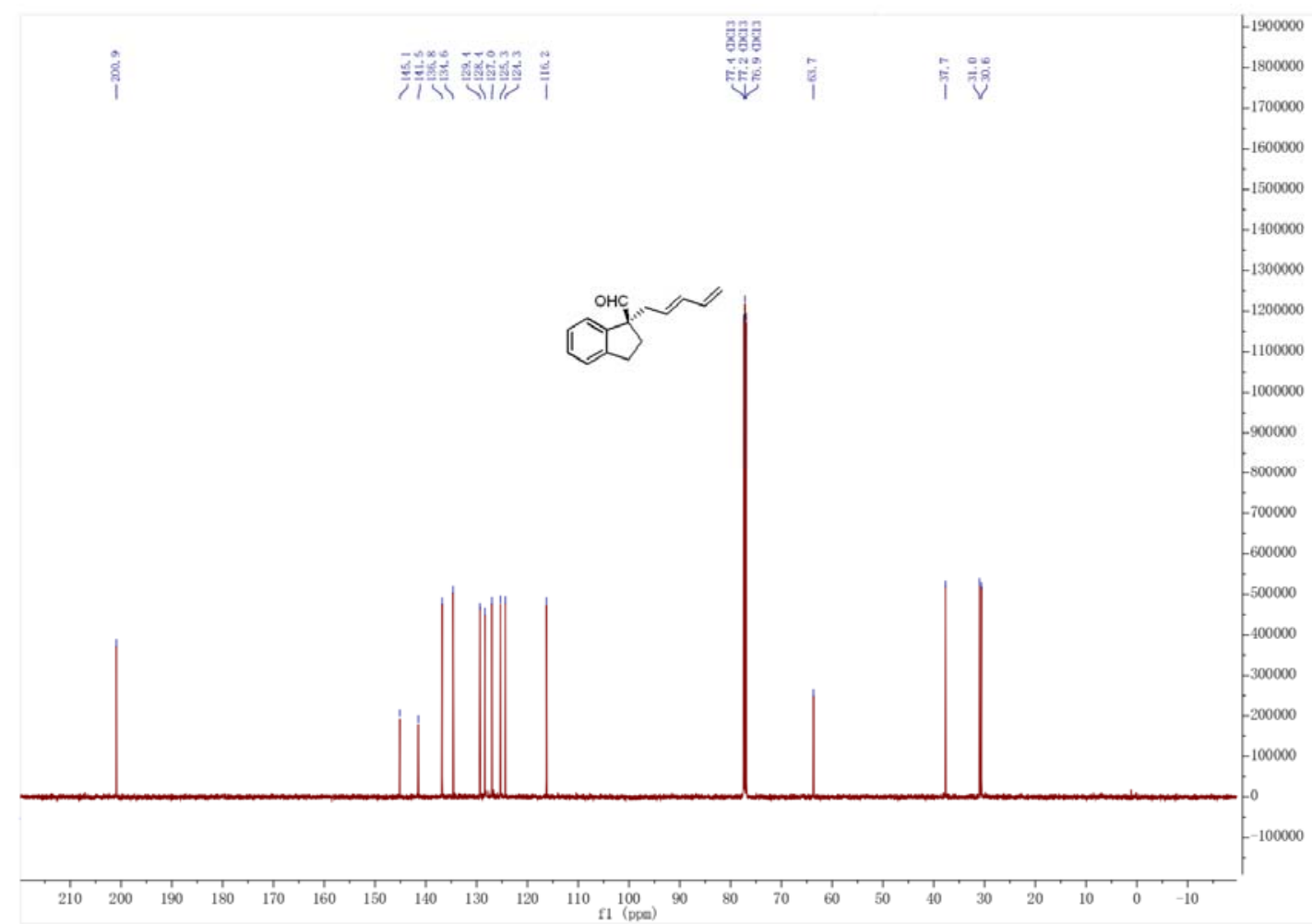


(R,E)-1-(penta-2,4-dien-1-yl)-1,2,3,4-tetrahydronaphthalene-1-carbaldehyde (3n) ${ }^{1} \mathbf{H}$ NMR (500 MHz, Chloroform-d)

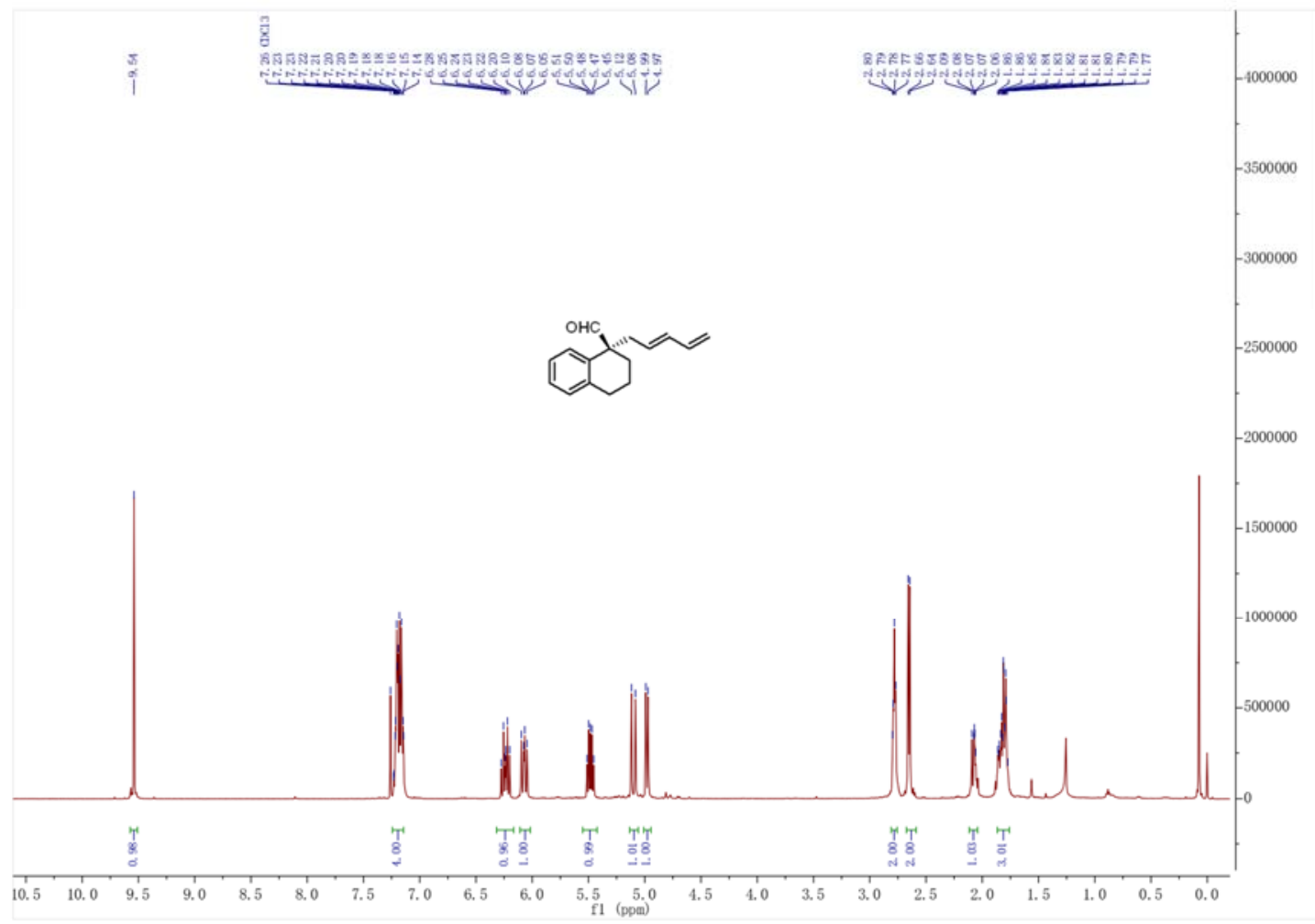

${ }^{13}$ C NMR (126 MHz, Chloroform-d)

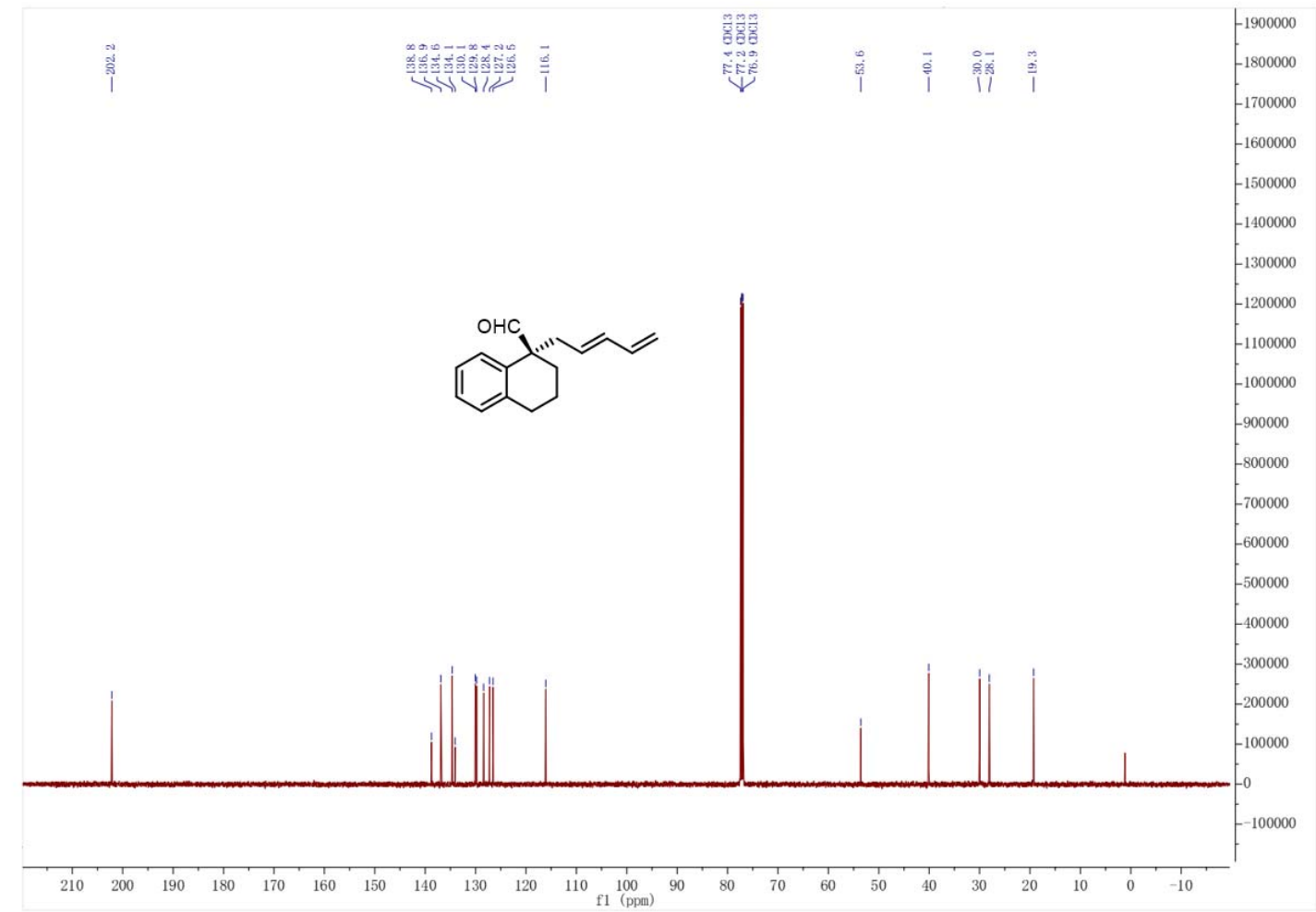


(R,E)-5-(penta-2,4-dien-1-yl)-6,7,8,9-tetrahydro-5H-benzo[7]annulene-5-carbaldehyde (3o) ${ }^{1}$ H NMR (500 MHz, Chloroform-d)

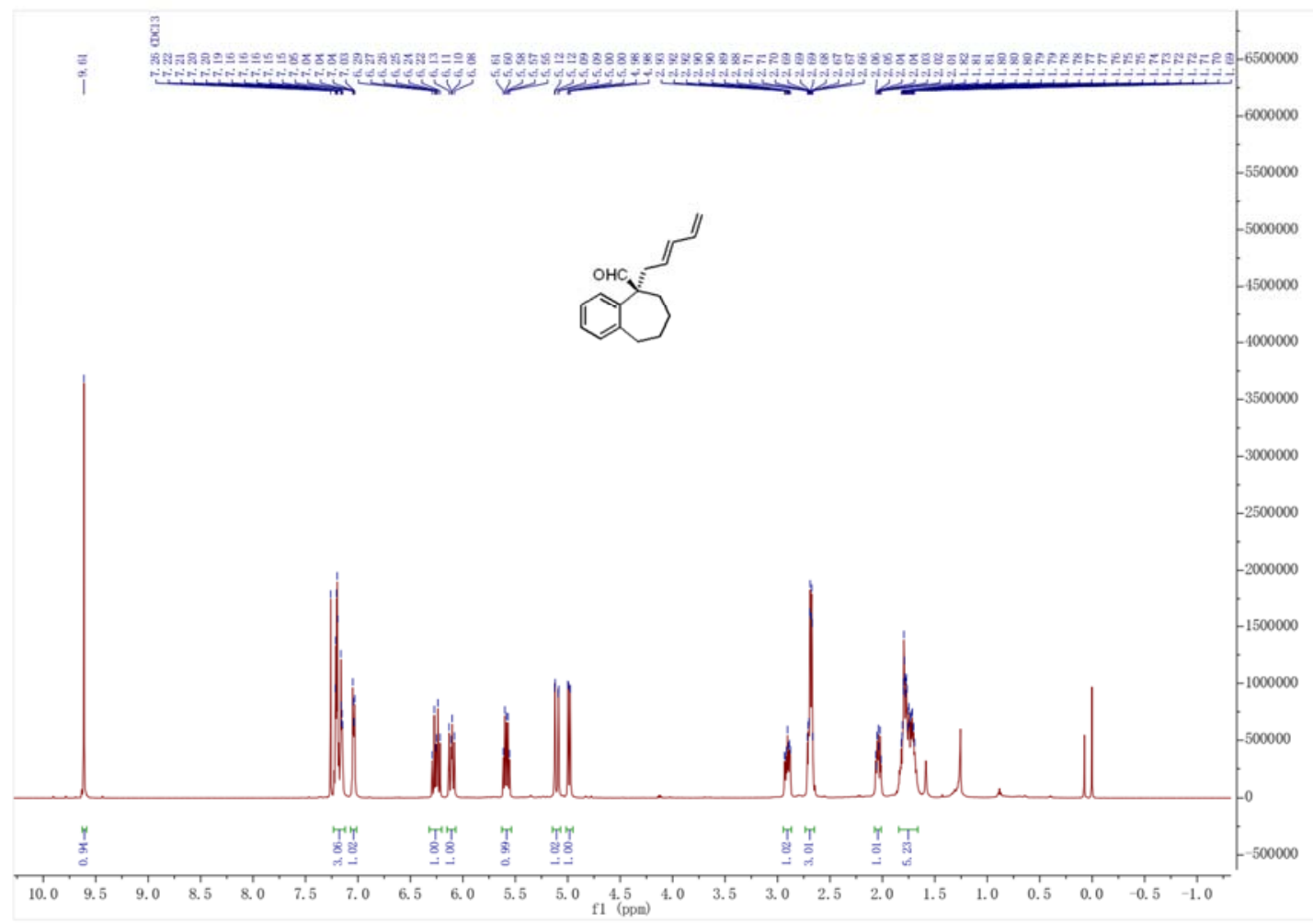

${ }^{13}$ C NMR (126 MHz, Chloroform- $d$ )

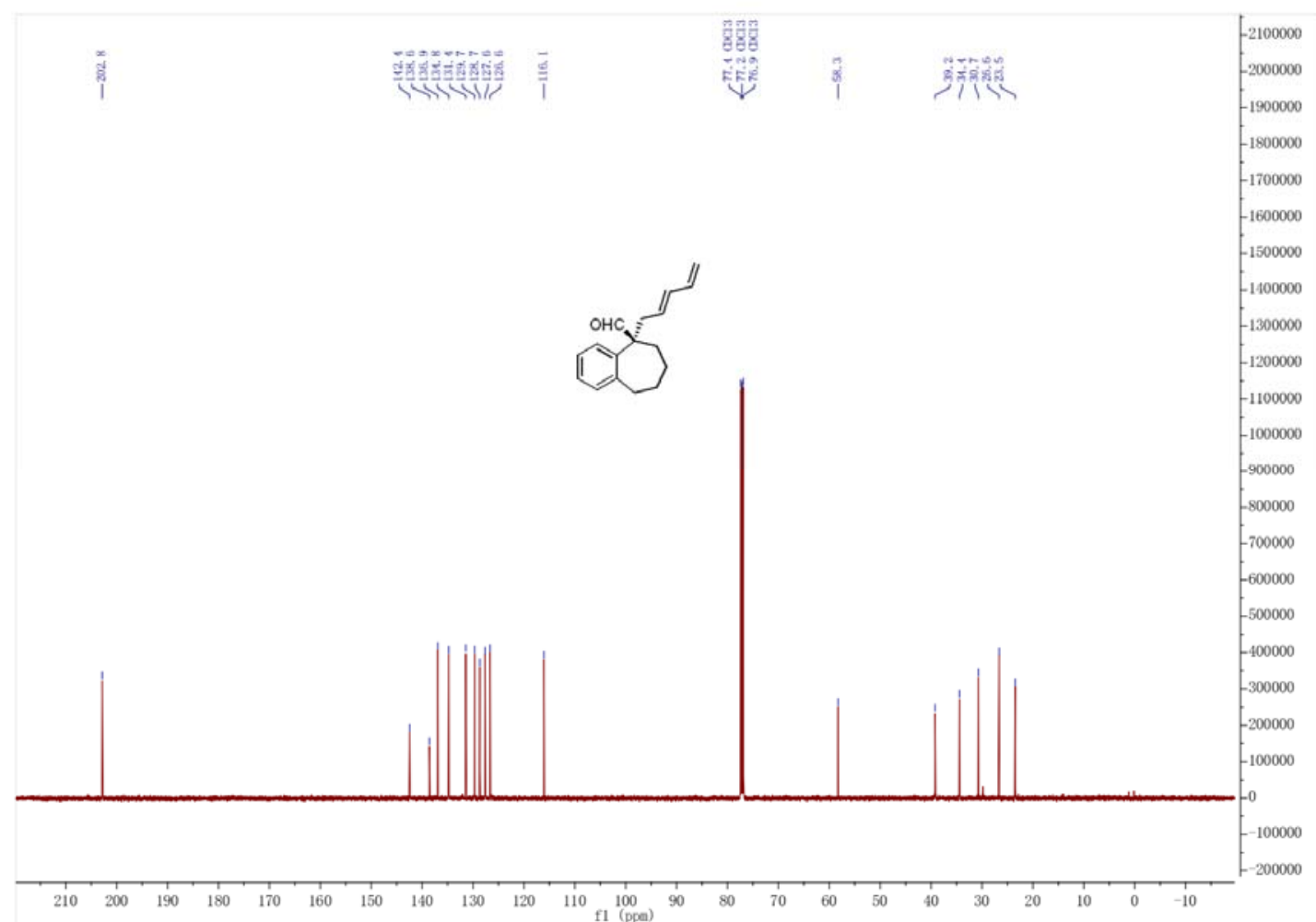


(R,E)-2-methyl-2-(pyridin-2-yl)hepta-4,6-dienal (3p)

${ }^{1}$ H NMR (400 MHz, Chloroform-d)

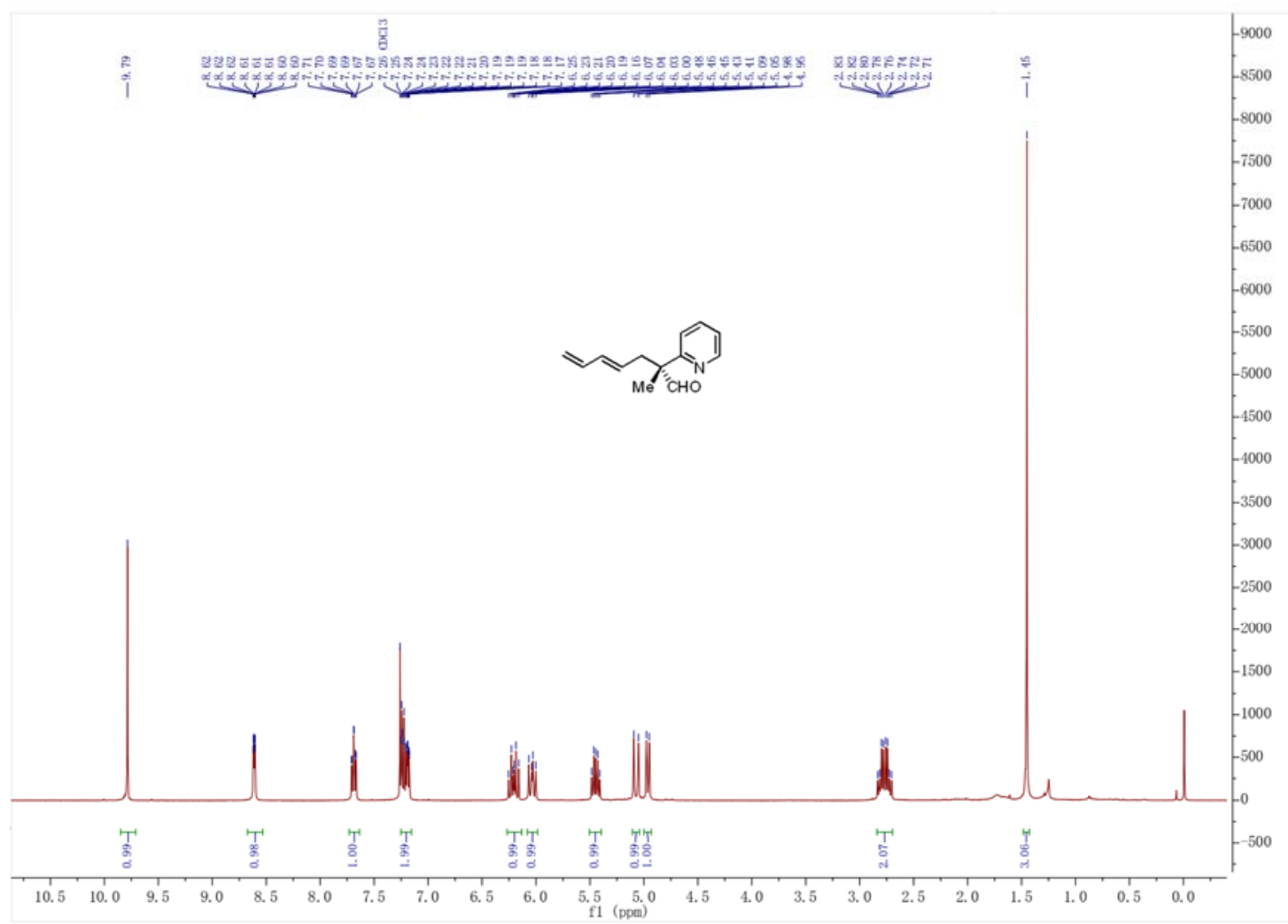

${ }^{13}$ C NMR (101 MHz, Chloroform-d)

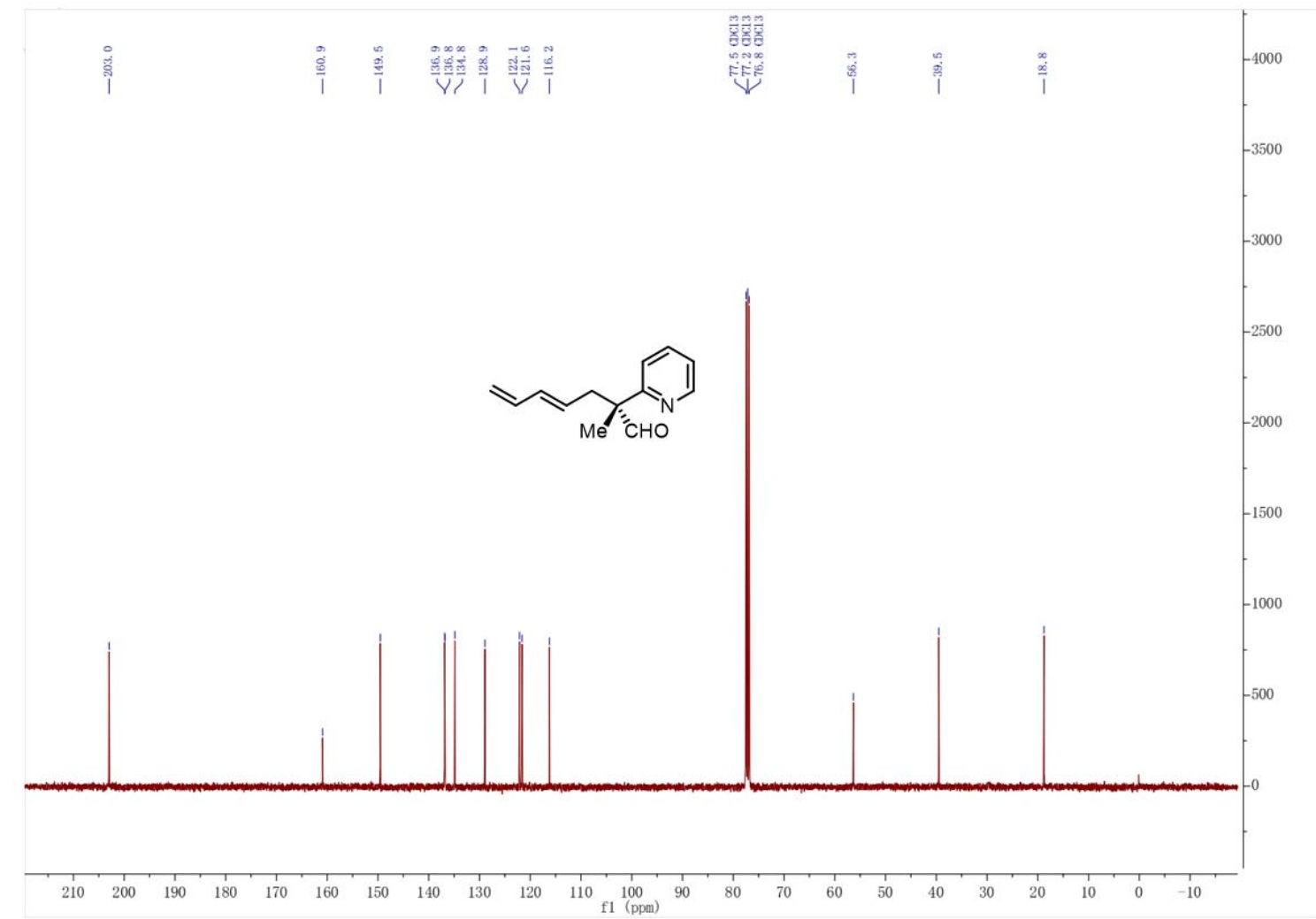


(S,E)-2-methyl-2-(thiophen-2-yl)hepta-4,6-dienal (3q)

${ }^{1}$ H NMR (400 MHz, Chloroform-d)

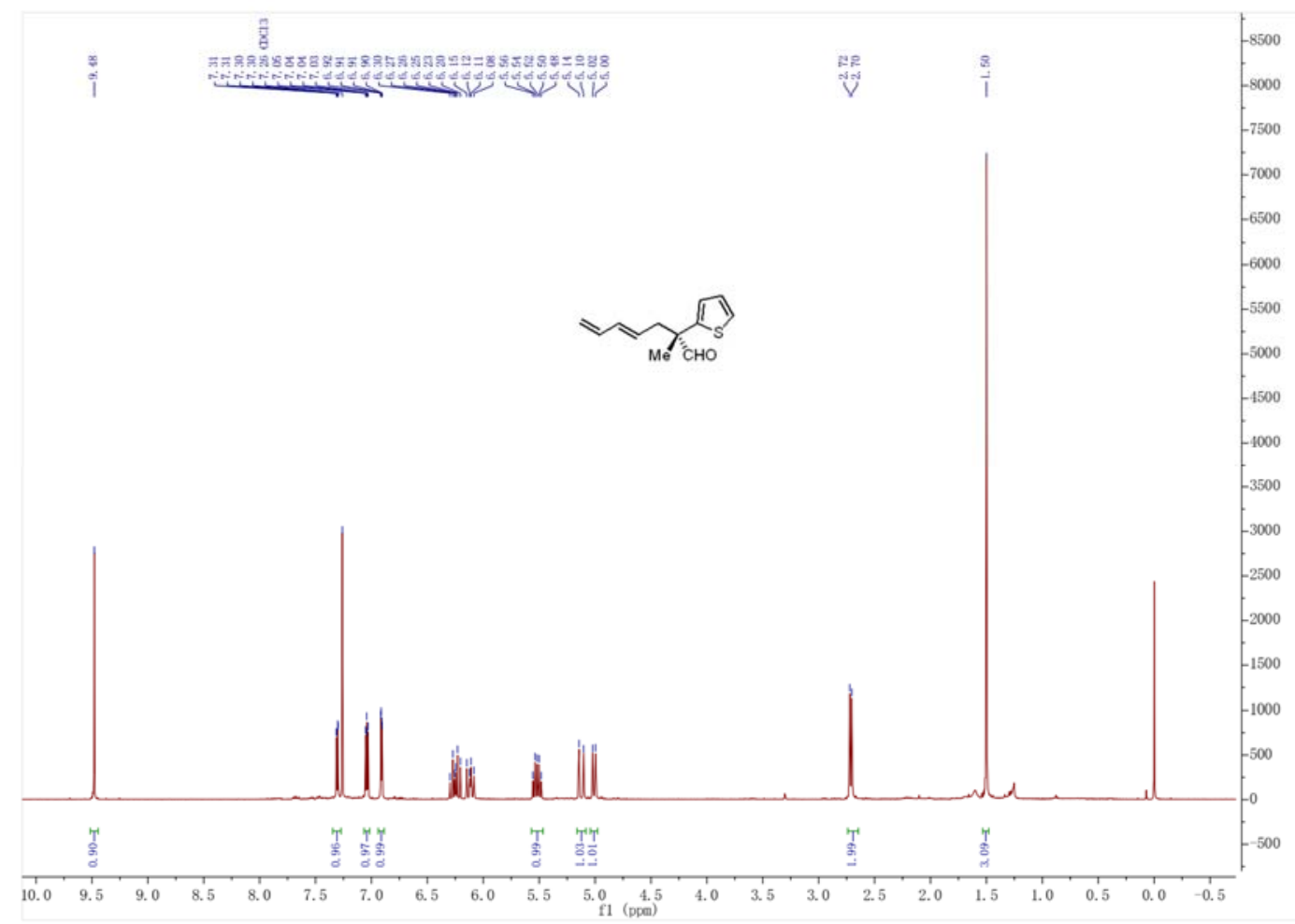

${ }^{13}$ C NMR (101 MHz, Chloroform-d)

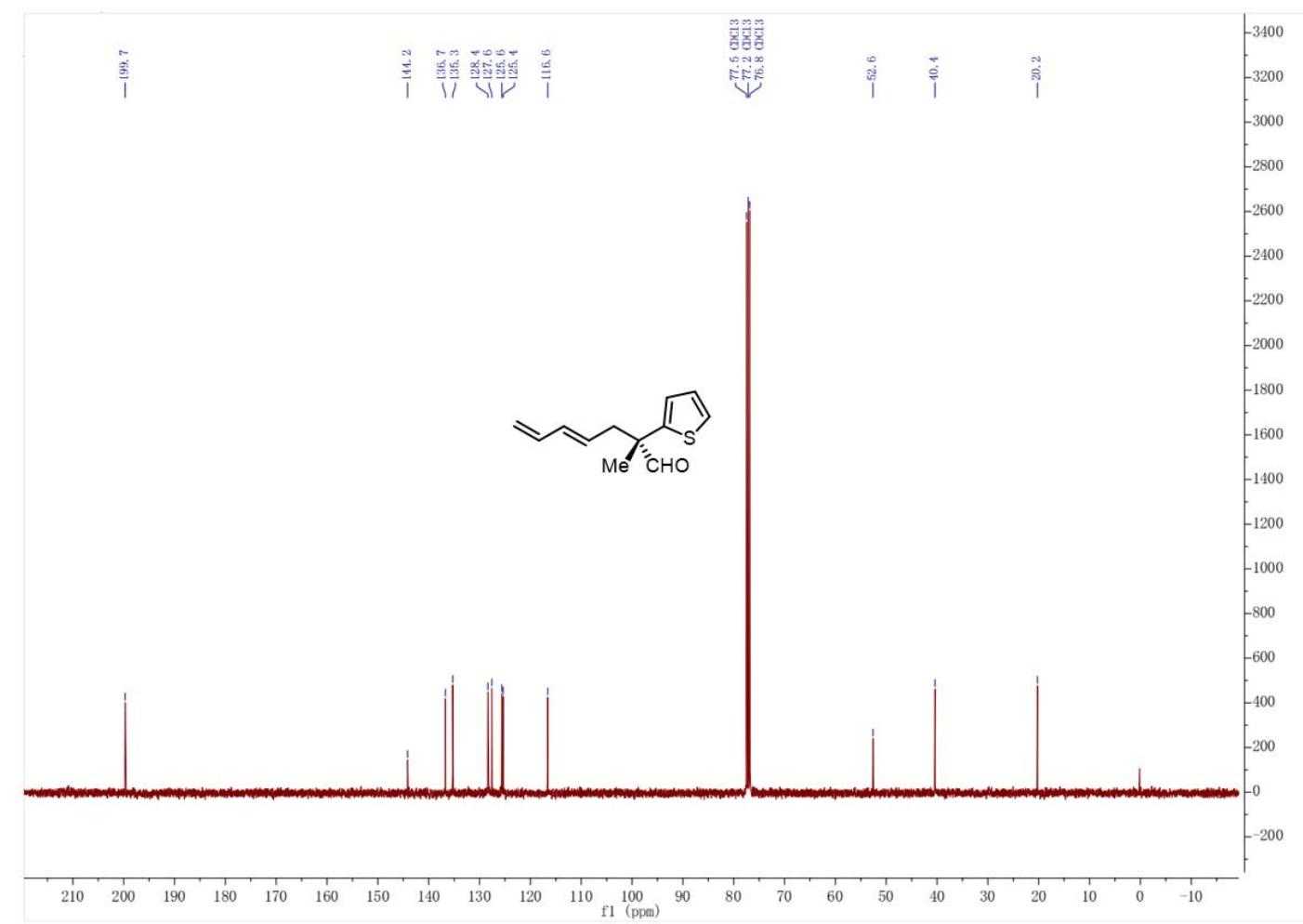


(R,4E,6E)-2-methyl-2,7-diphenylhepta-4,6-dienal (3r)

${ }^{1}$ H NMR (500 MHz, Chloroform-d)

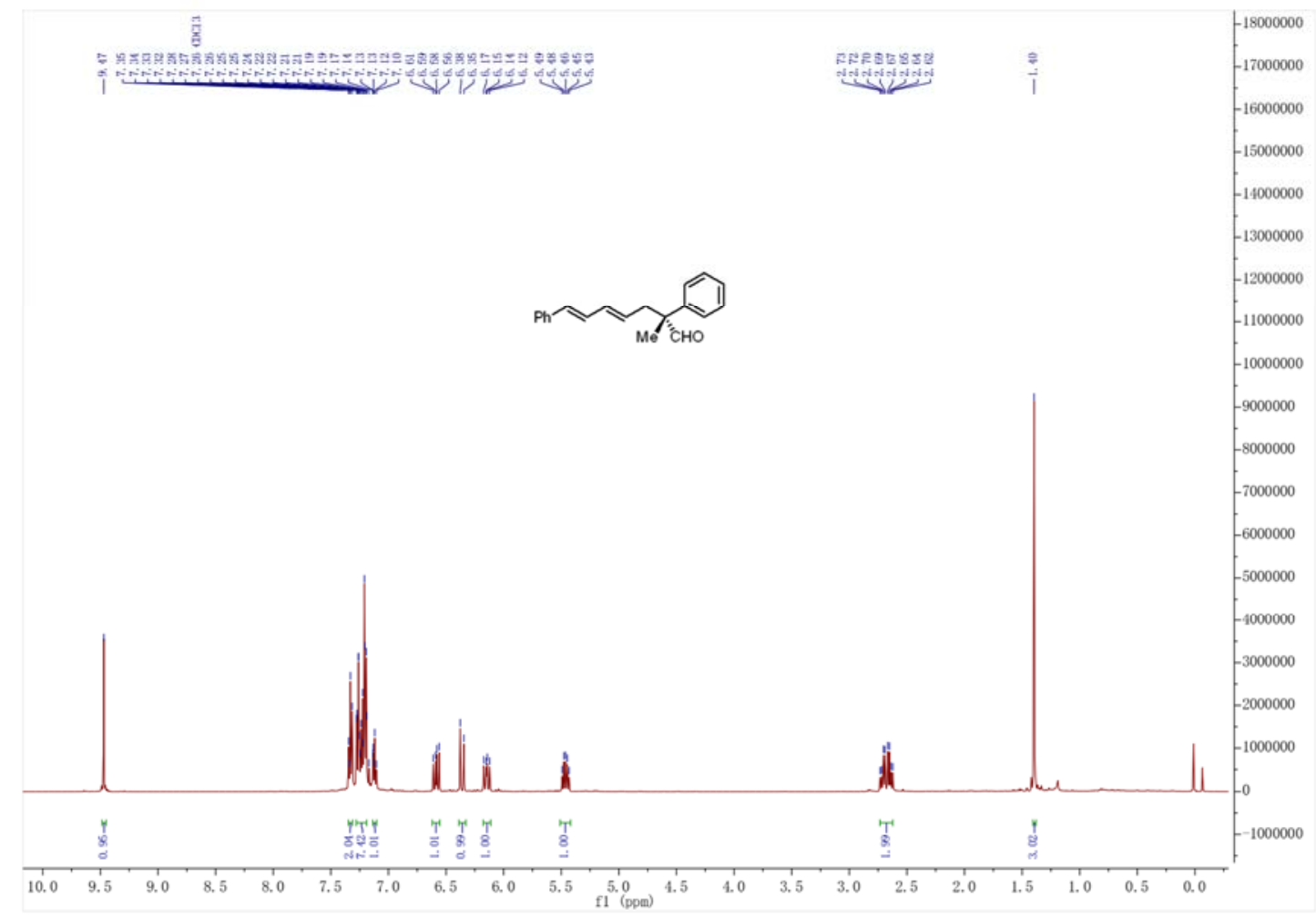

${ }^{13}$ C NMR (126 MHz, Chloroform-d)

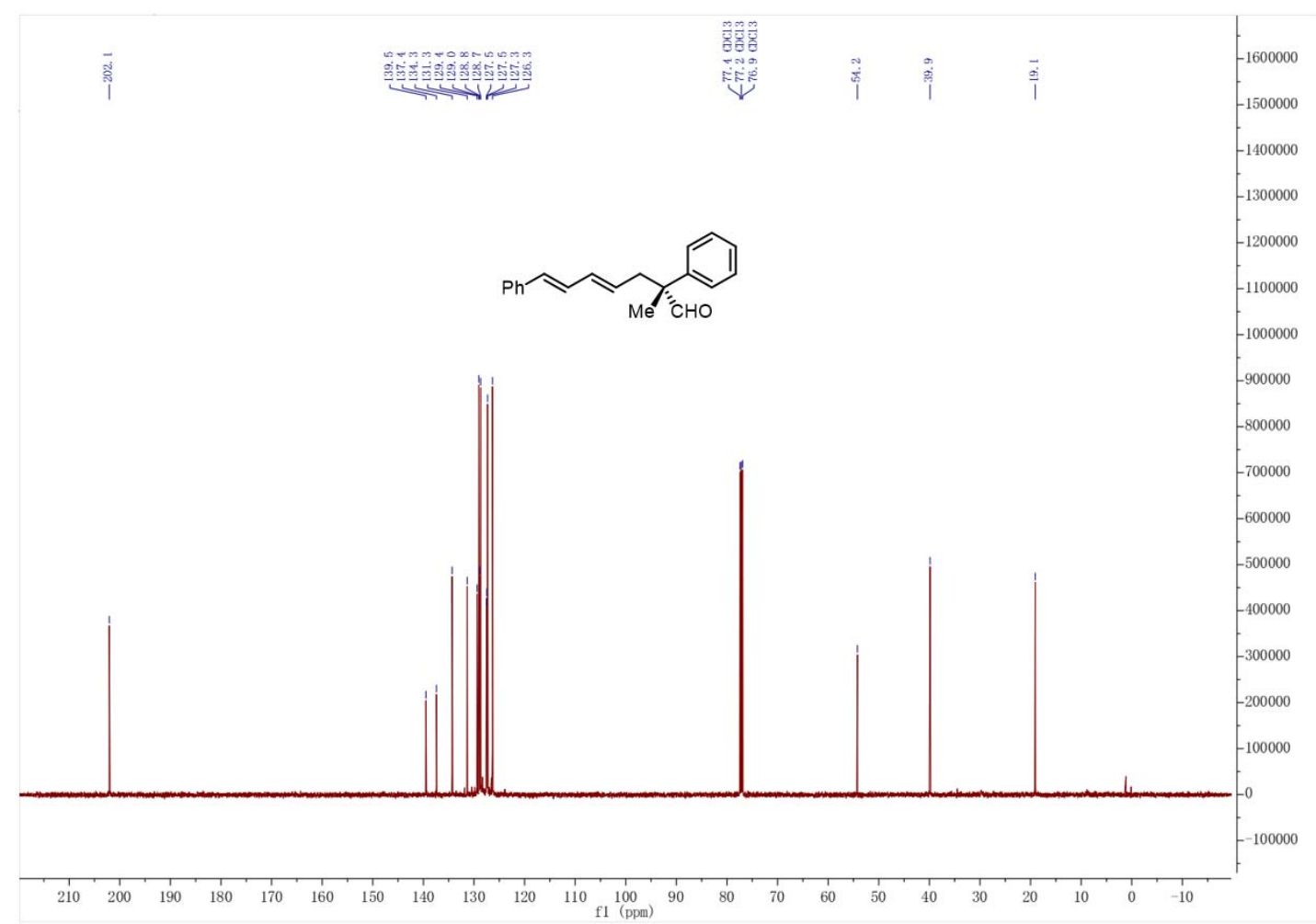


(R,4E,6E)-2-methyl-2-phenyl-7-(p-tolyl)hepta-4,6-dienal (3s)

${ }^{1}$ H NMR (500 MHz, Chloroform-d)

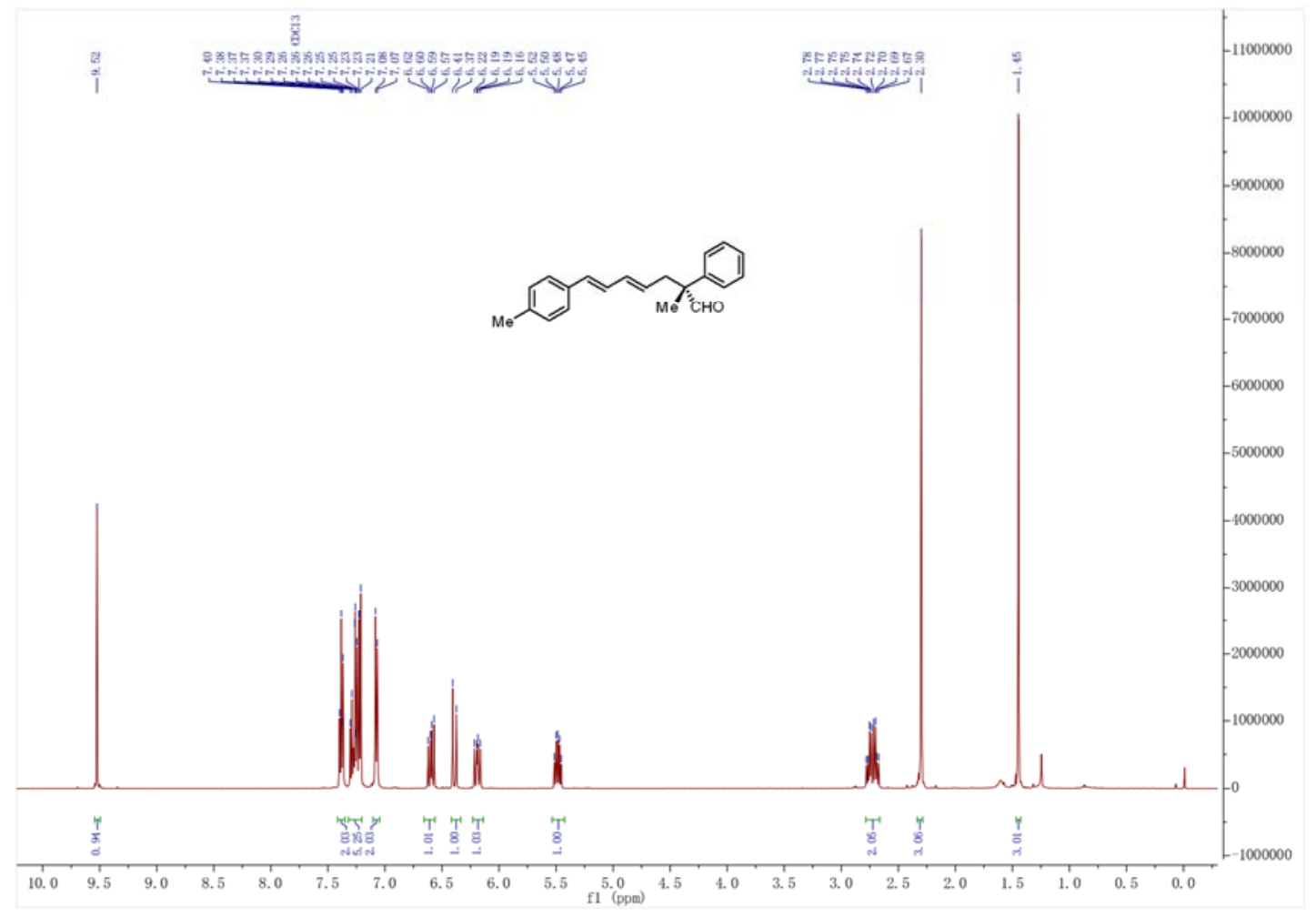

${ }^{13}$ C NMR (126 MHz, Chloroform-d)

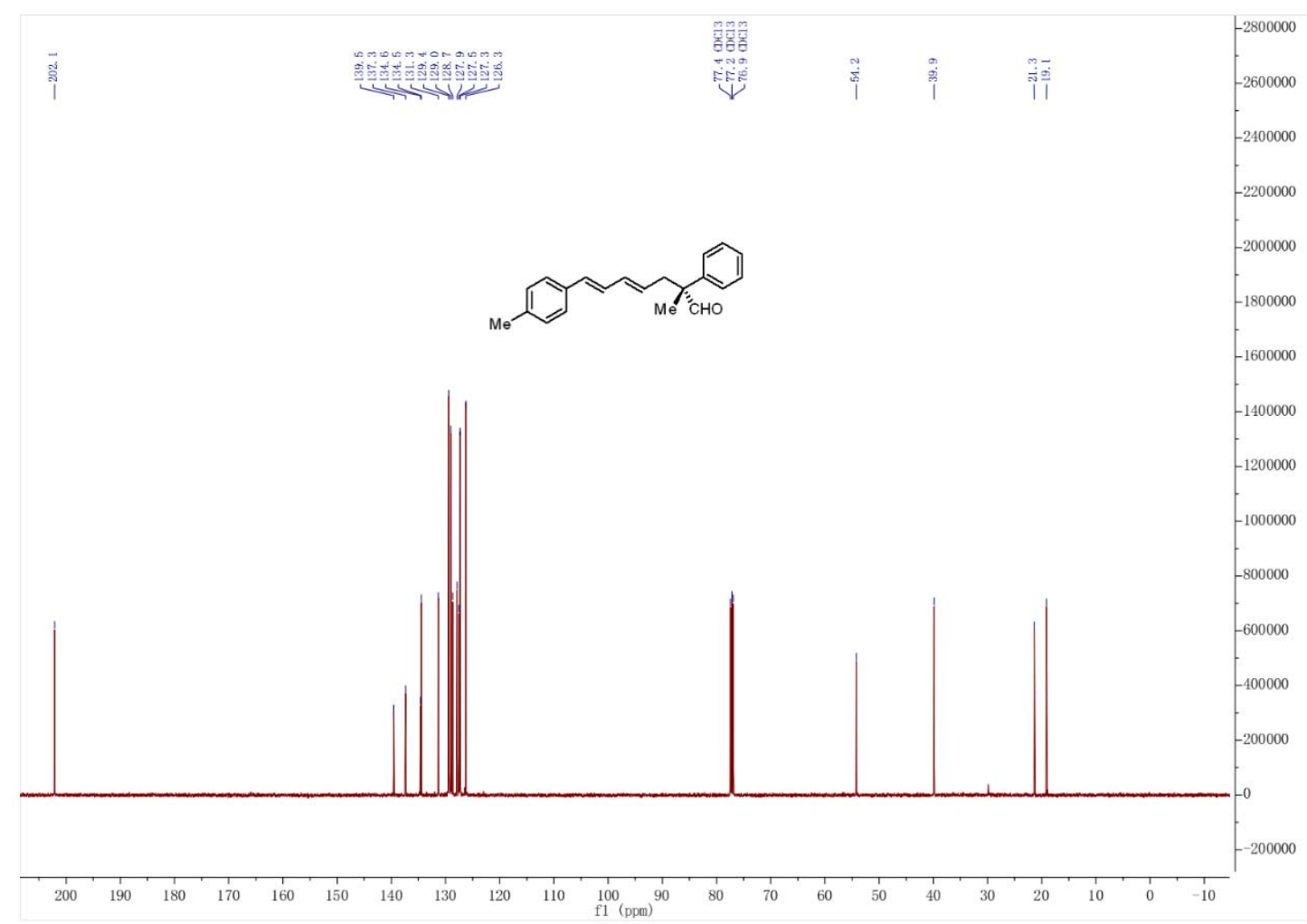


(R,4E,6E)-7-(4-fluorophenyl)-2-methyl-2-phenylhepta-4,6-dienal (3t)

${ }^{1}$ H NMR (500 MHz, Chloroform-d)

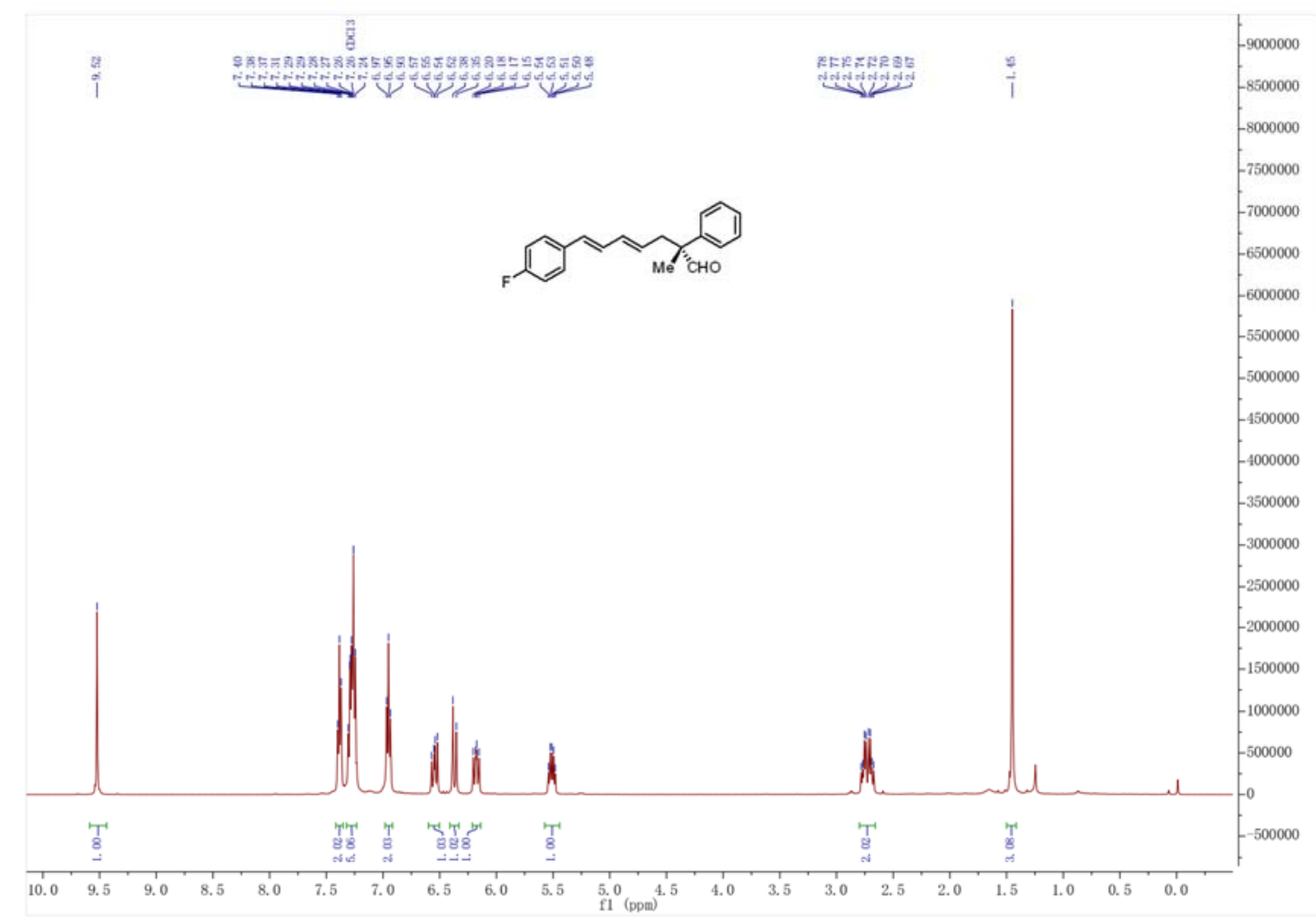

${ }^{13}$ C NMR (126 MHz, Chloroform-d)

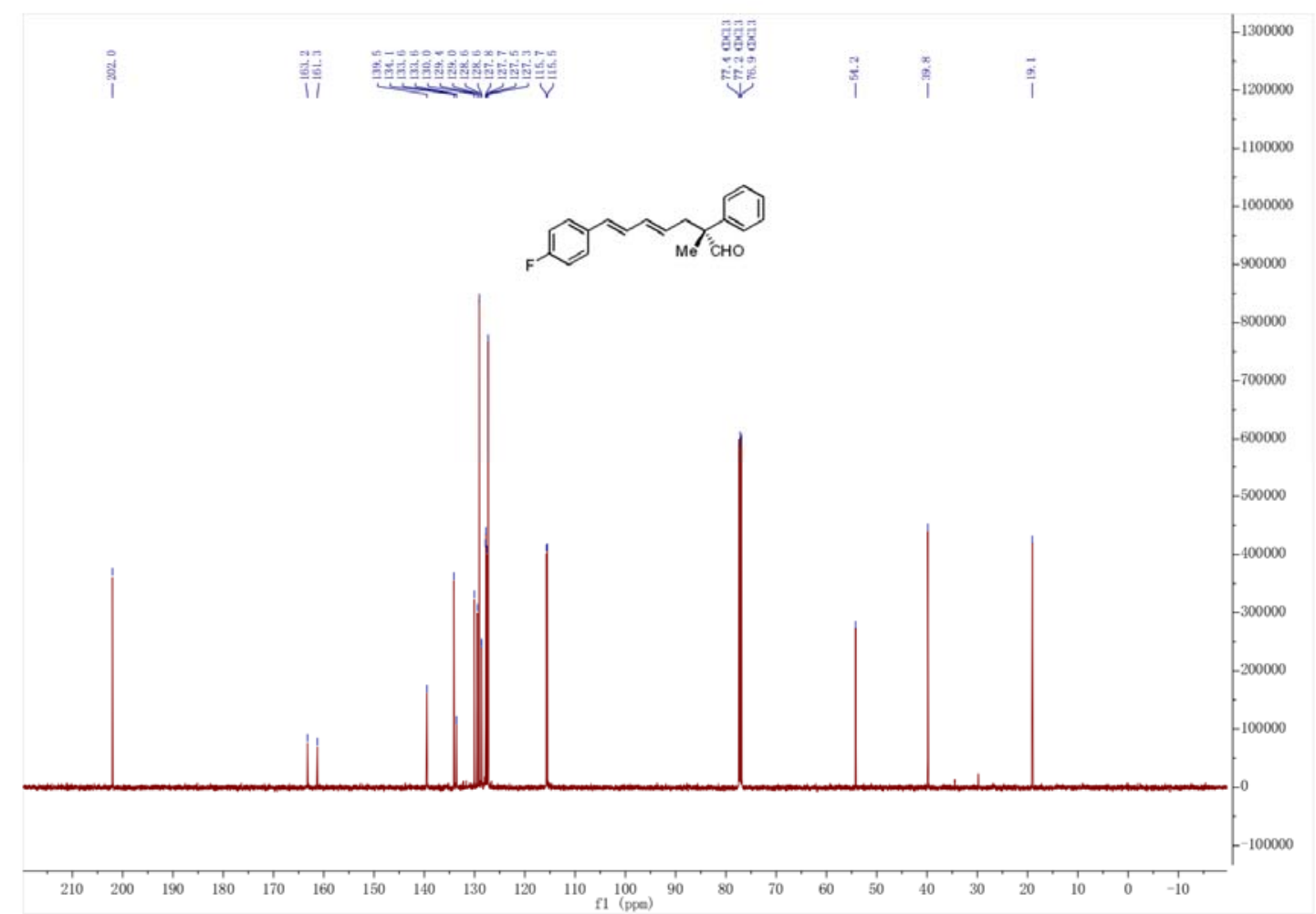


${ }^{19}$ F NMR (376 MHz, Chloroform-d)

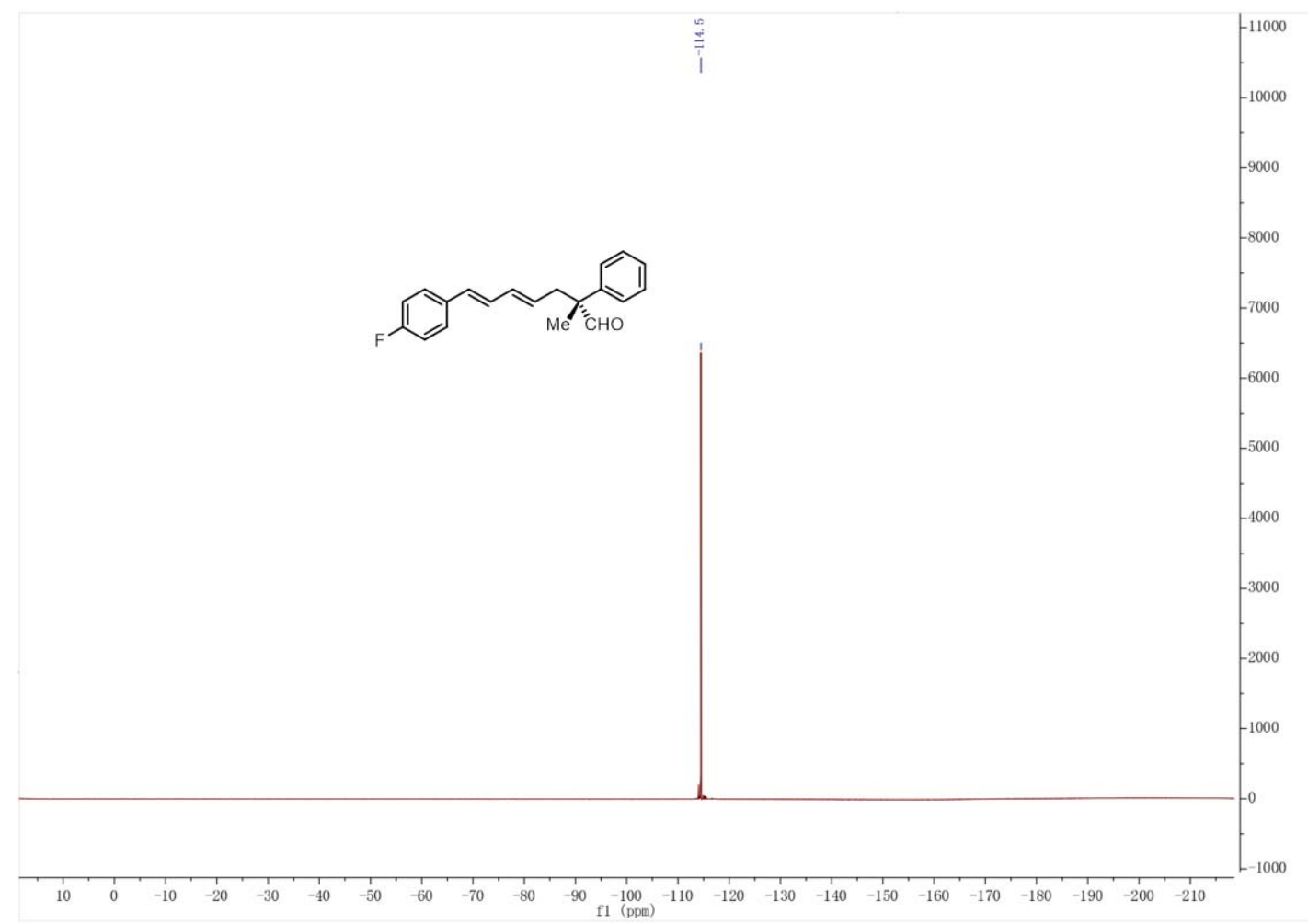

(R,4E,6E)-7-(4-chlorophenyl)-2-methyl-2-phenylhepta-4,6-dienal (3u)

${ }^{1}$ H NMR (400 MHz, Chloroform-d)

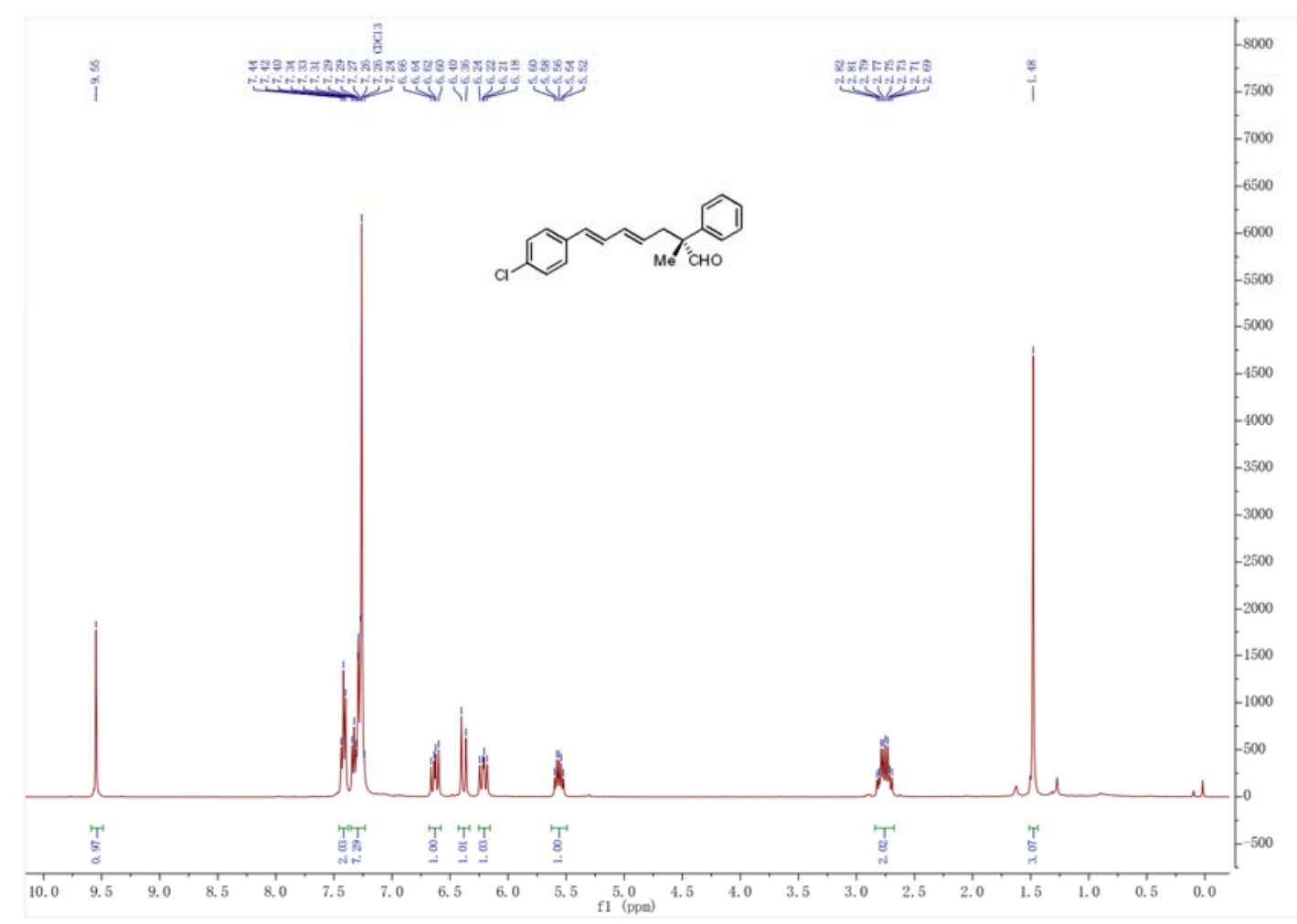


${ }^{13}$ C NMR (126 MHz, Chloroform-d)

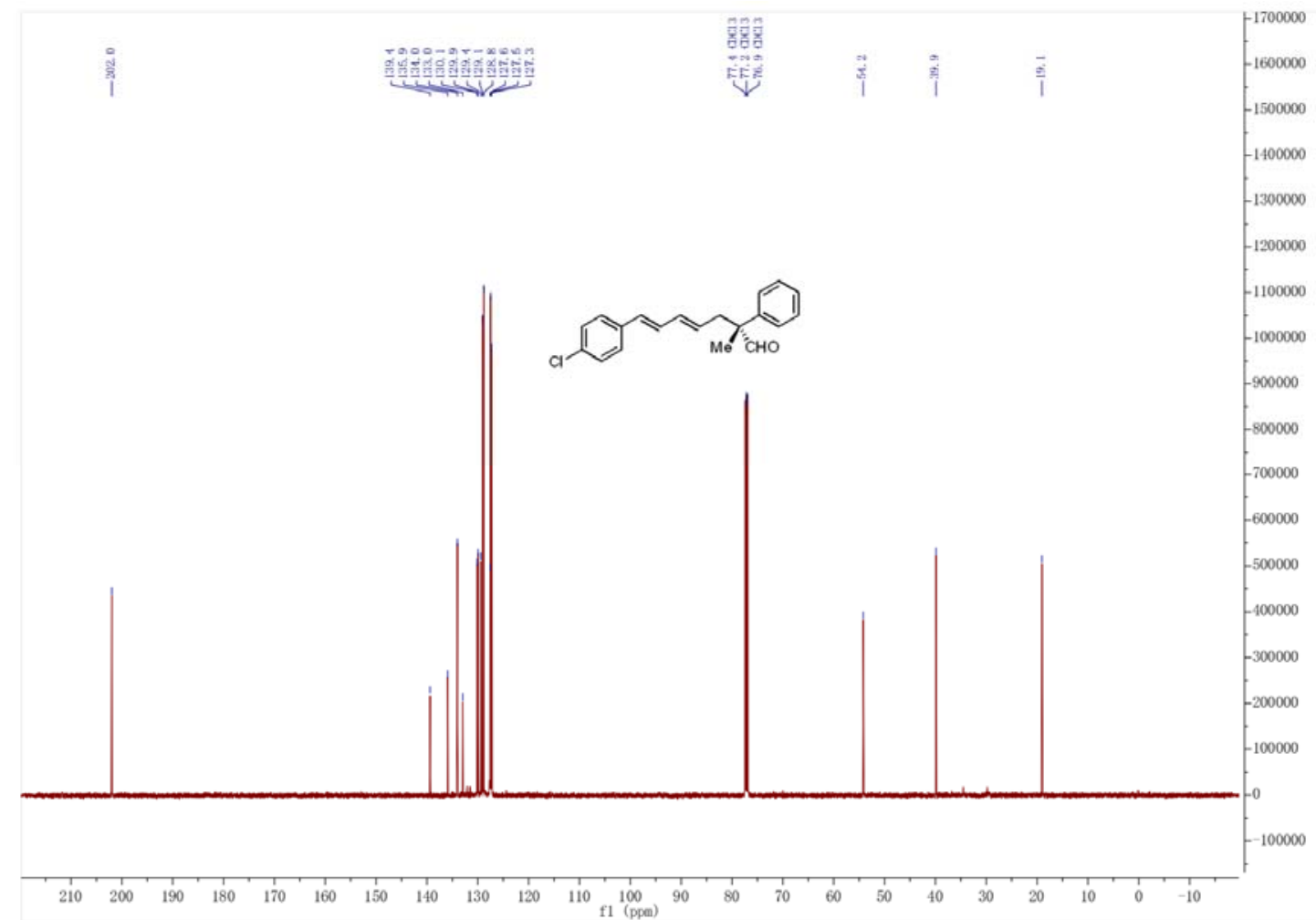

(R,4E,6E)-2-methyl-2-phenyl-7-(4-(trifluoromethyl)phenyl)hepta-4,6-dienal (3v) ${ }^{1}$ H NMR (400 MHz, Chloroform-d)

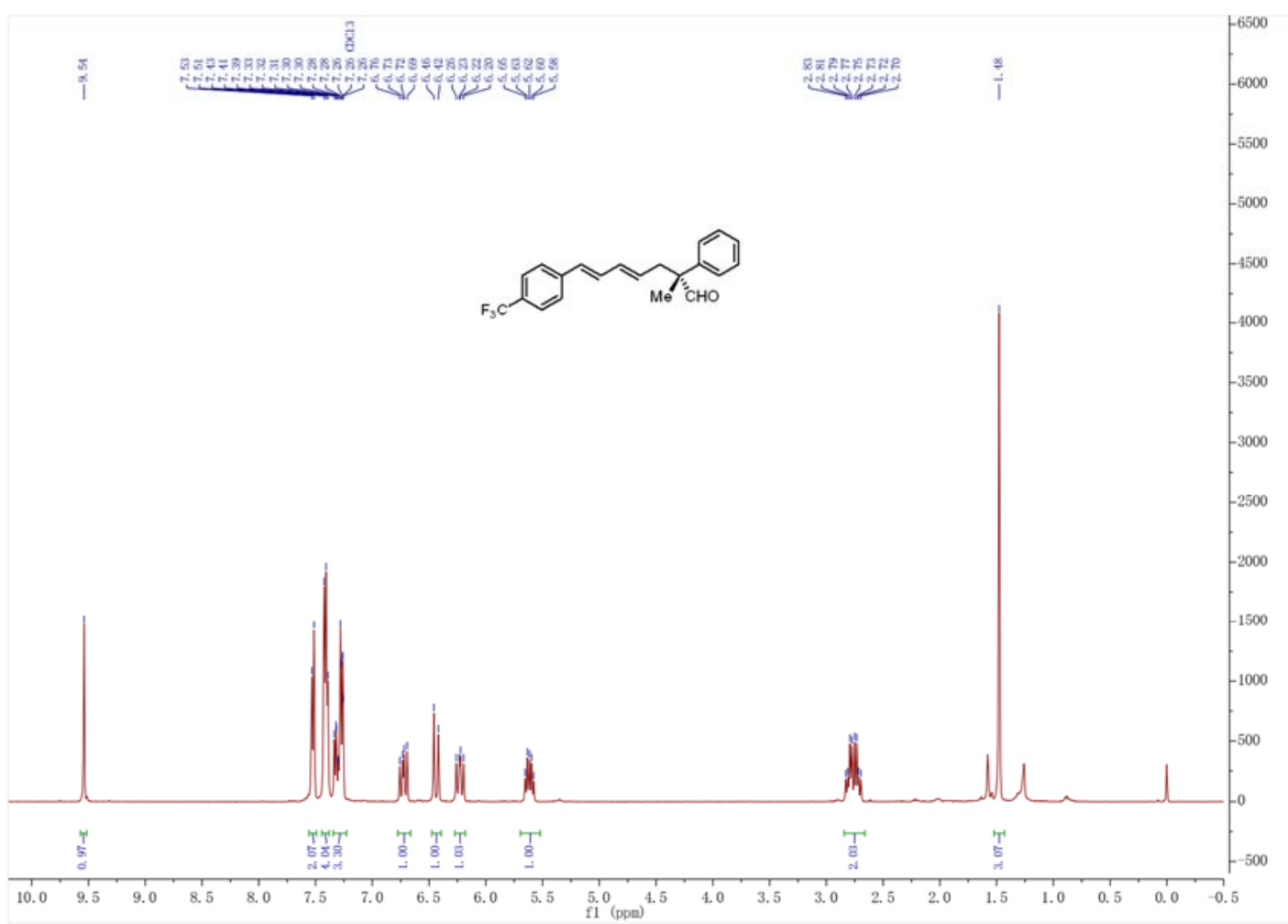


${ }^{13}$ C NMR (126 MHz, Chloroform-d)

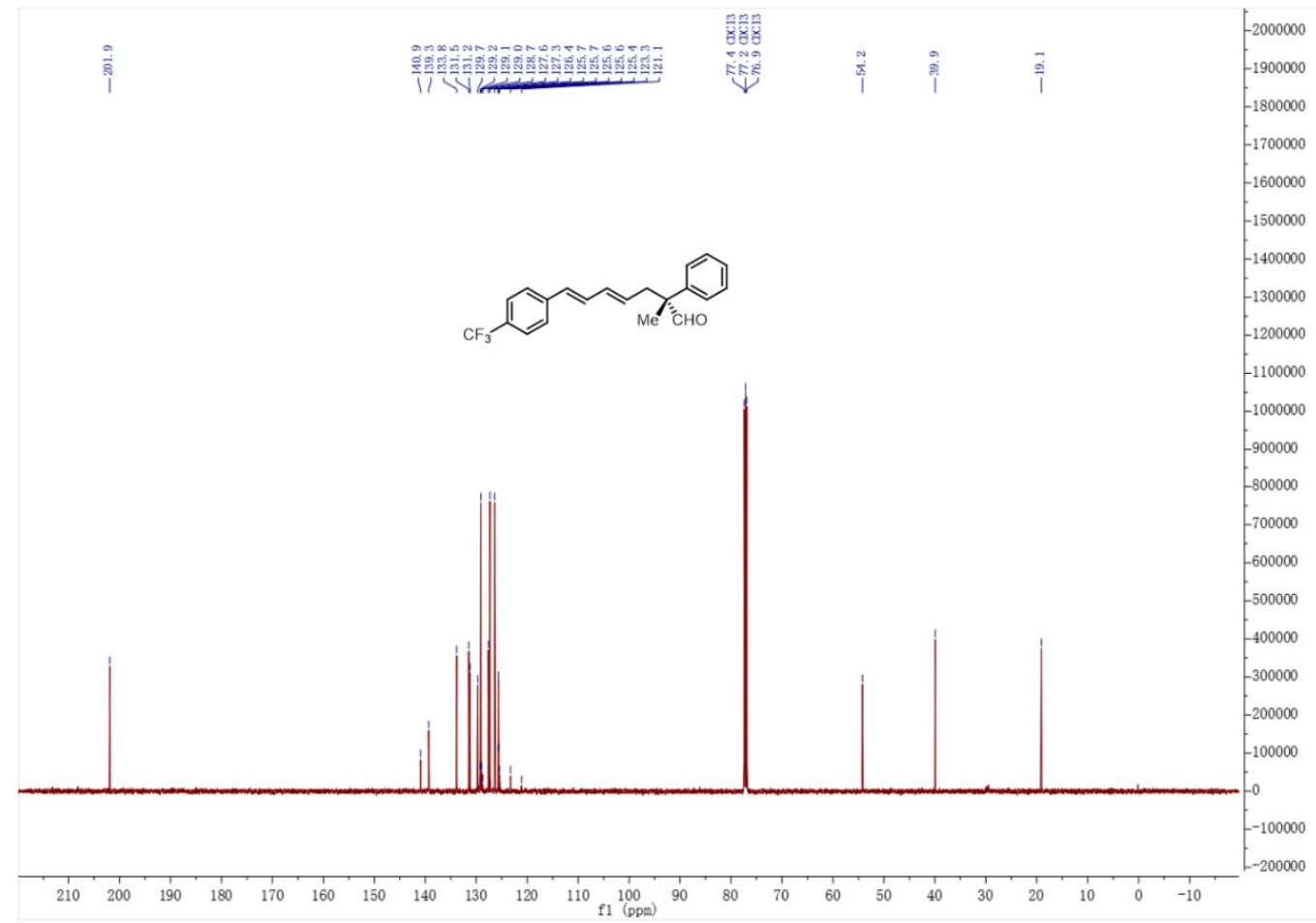

${ }^{19}$ F NMR (376 MHz, Chloroform-d)

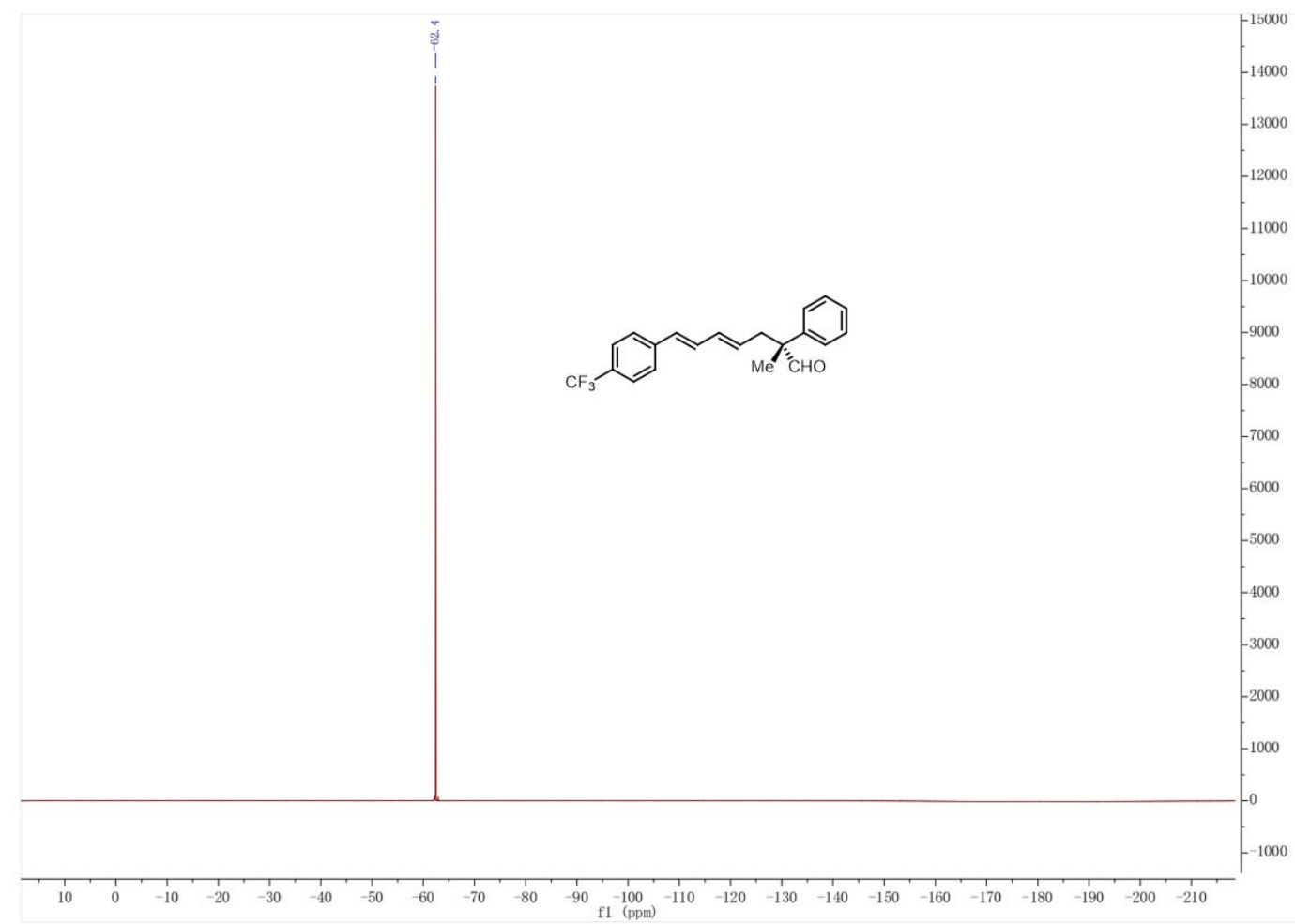


(R,4E,6E)-7-(3-fluorophenyl)-2-methyl-2-phenylhepta-4,6-dienal (3w)

${ }^{1}$ H NMR (400 MHz, Chloroform-d)

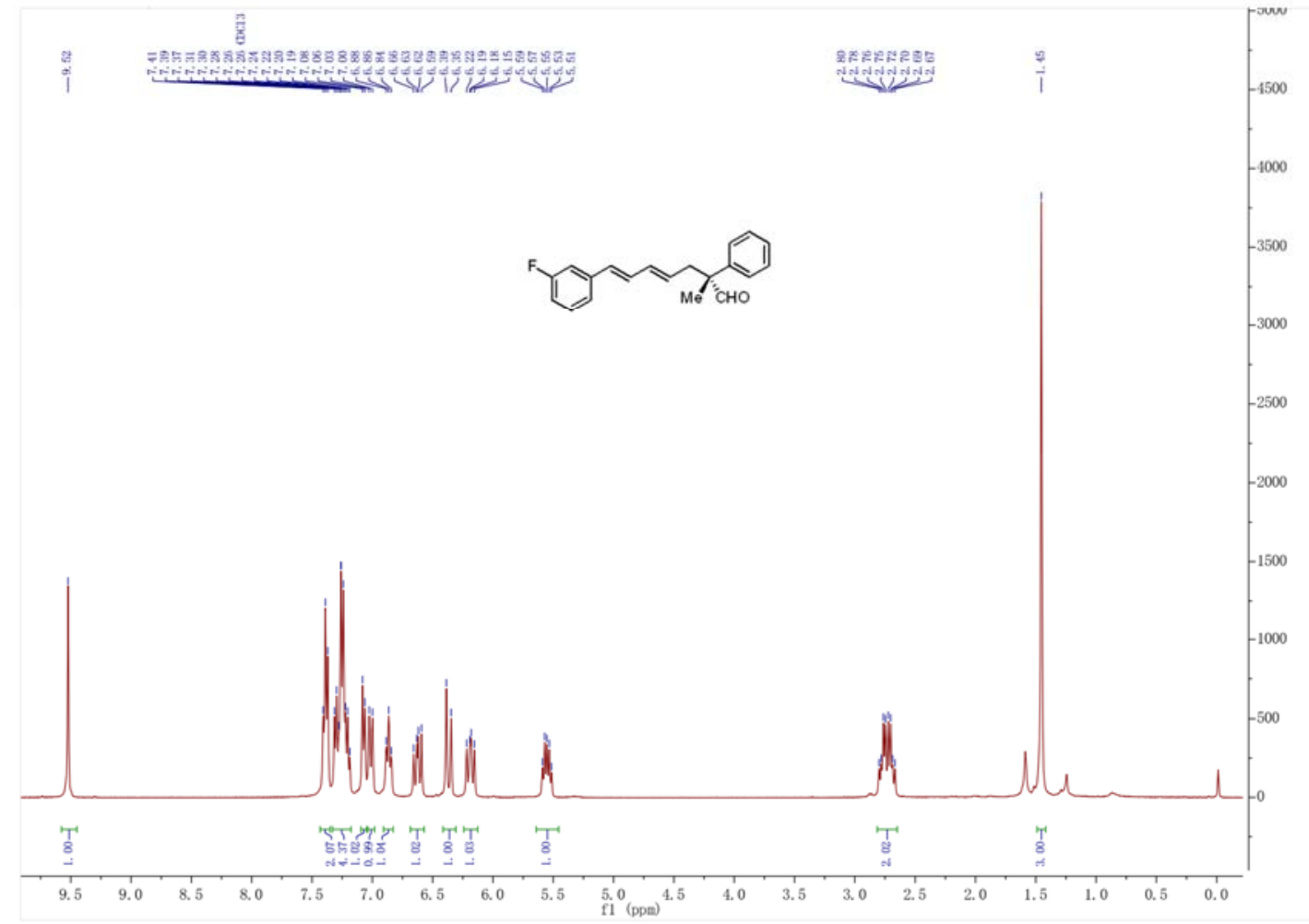

${ }^{13}$ C NMR (126 MHz, Chloroform-d)

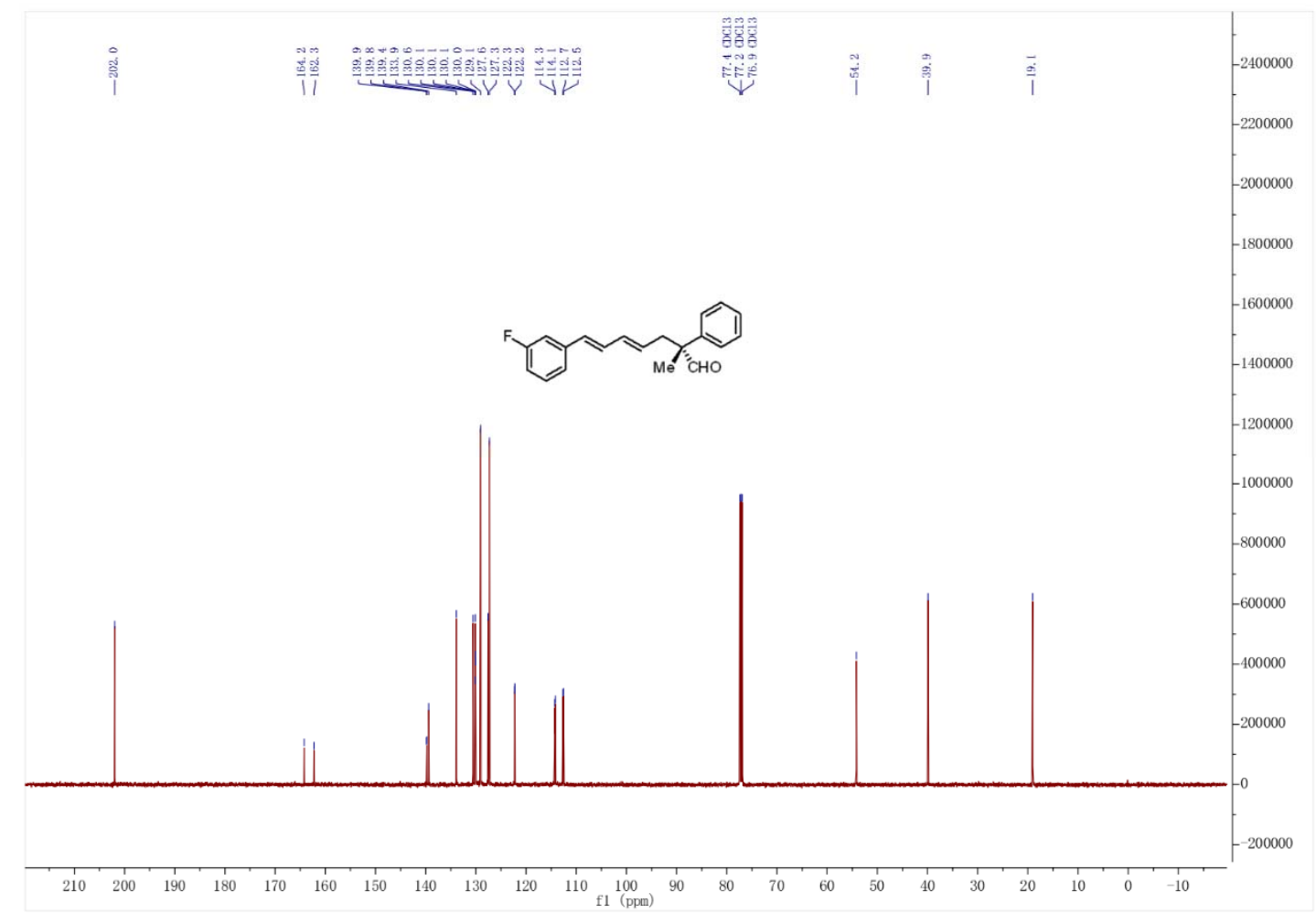


${ }^{19}$ F NMR (471 MHz, Chloroform-d)

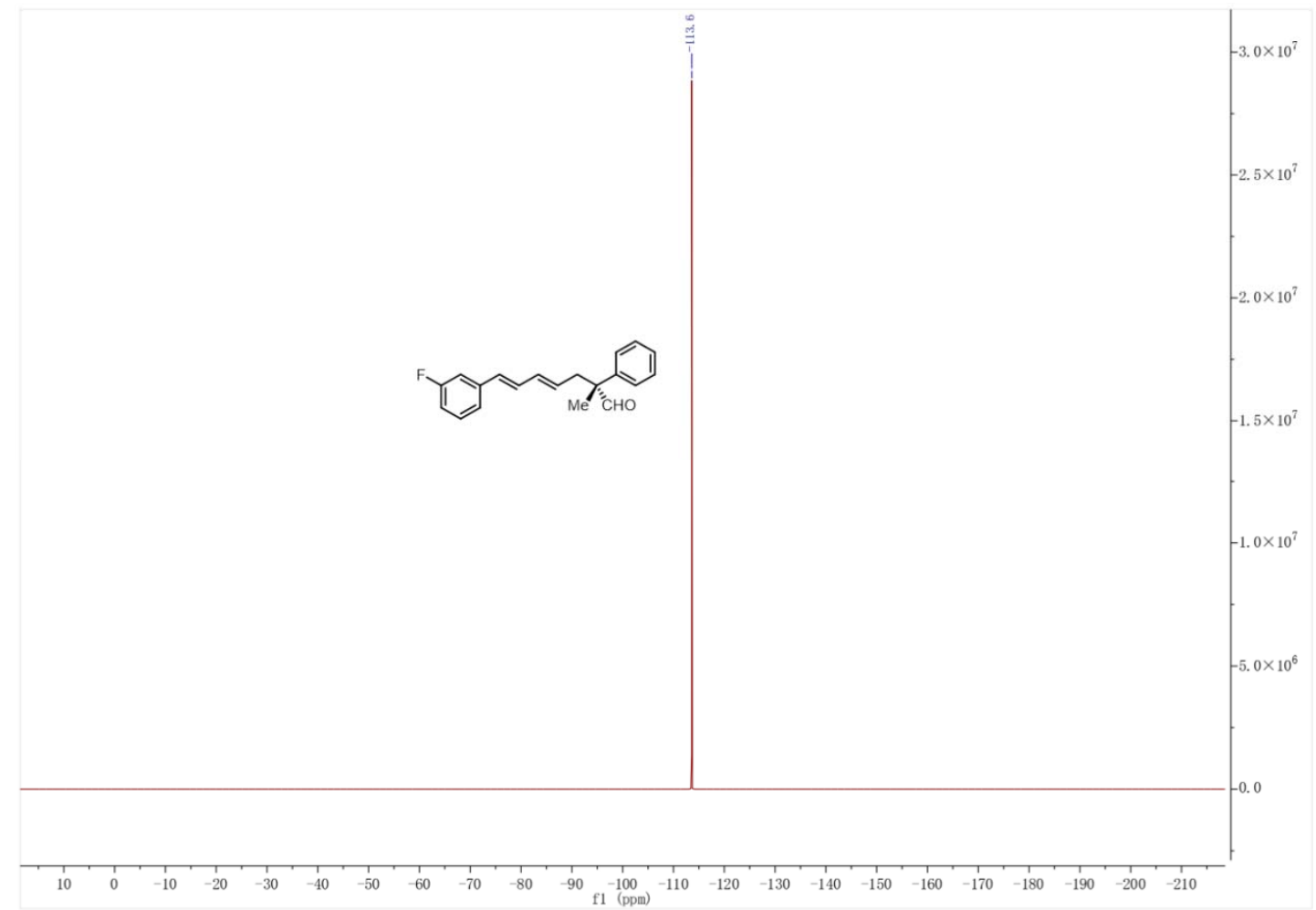

(R,4E,6E)-7-(3-chlorophenyl)-2-methyl-2-phenylhepta-4,6-dienal (3x)

${ }^{1}$ H NMR (500 MHz, Chloroform-d)

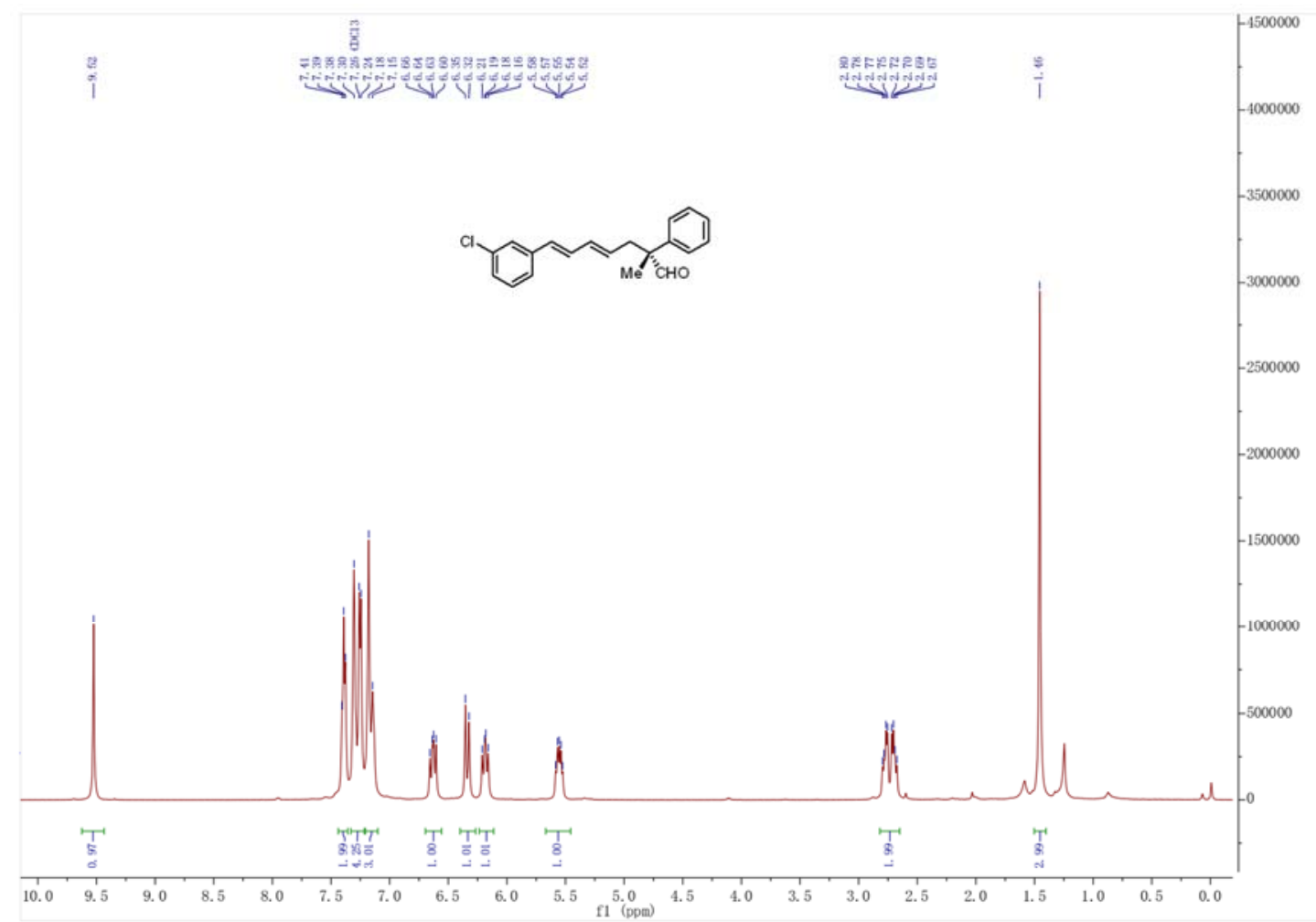


${ }^{13}$ C NMR (126 MHz, Chloroform-d)

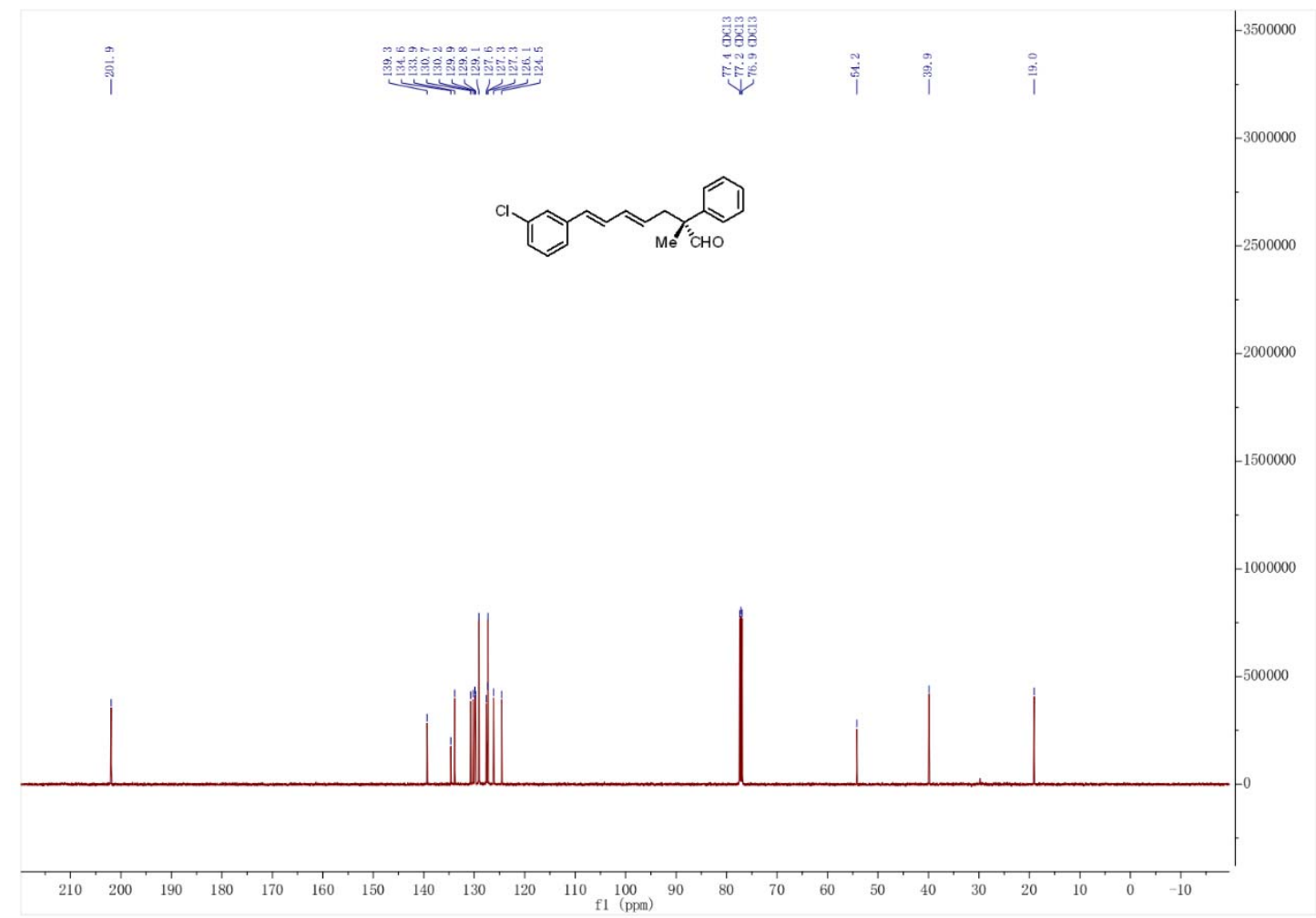

(R,4E,6E)-2-methyl-2-phenyl-7-(m-tolyl)hepta-4,6-dienal (3y)

${ }^{1}$ H NMR (400 MHz, Chloroform-d)

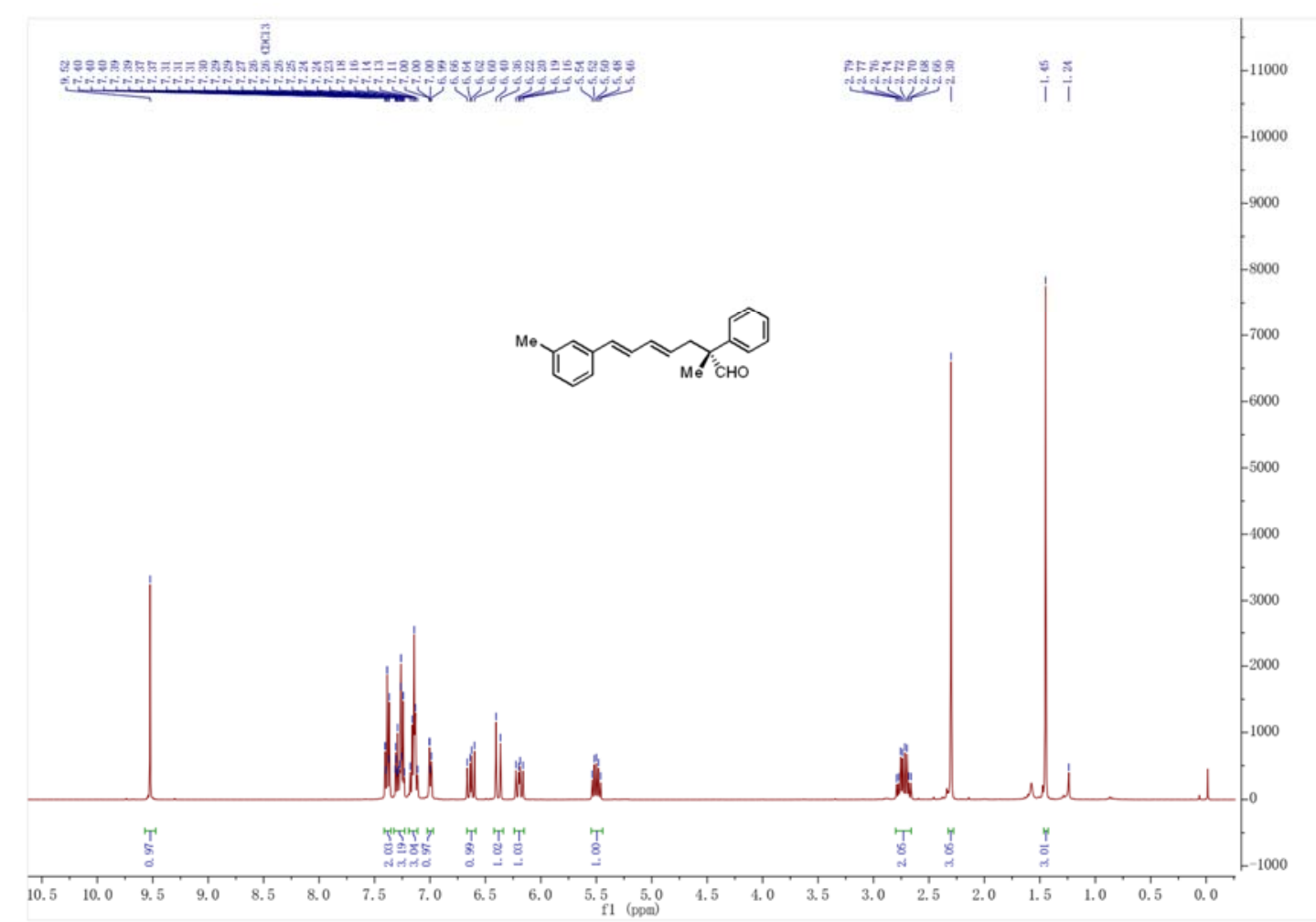


${ }^{13}$ C NMR (101 MHz, Chloroform-d)

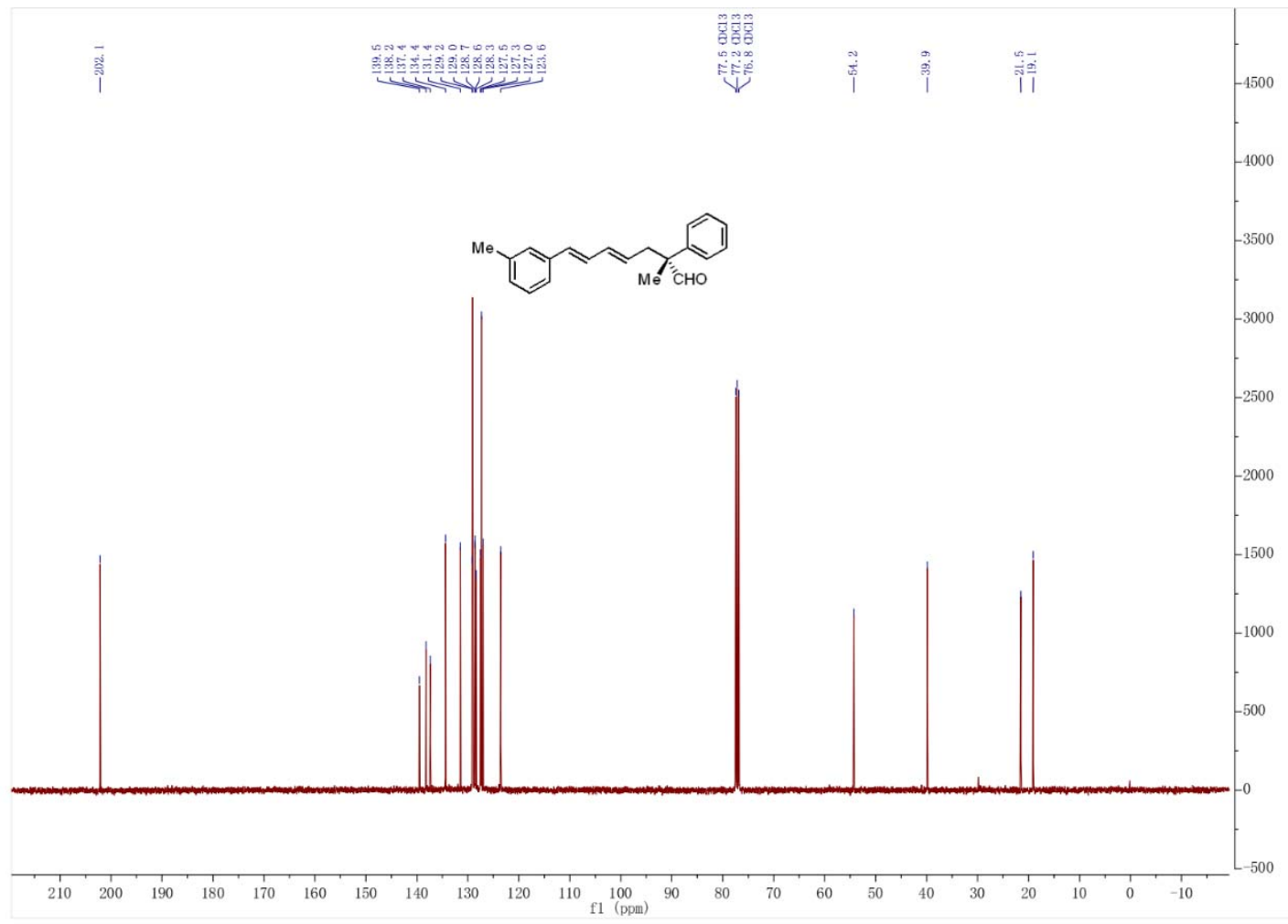

(R,4E,6E)-7-(3-methoxyphenyl)-2-methyl-2-phenylhepta-4,6-dienal (3z)

${ }^{1}$ H NMR (500 MHz, Chloroform-d)

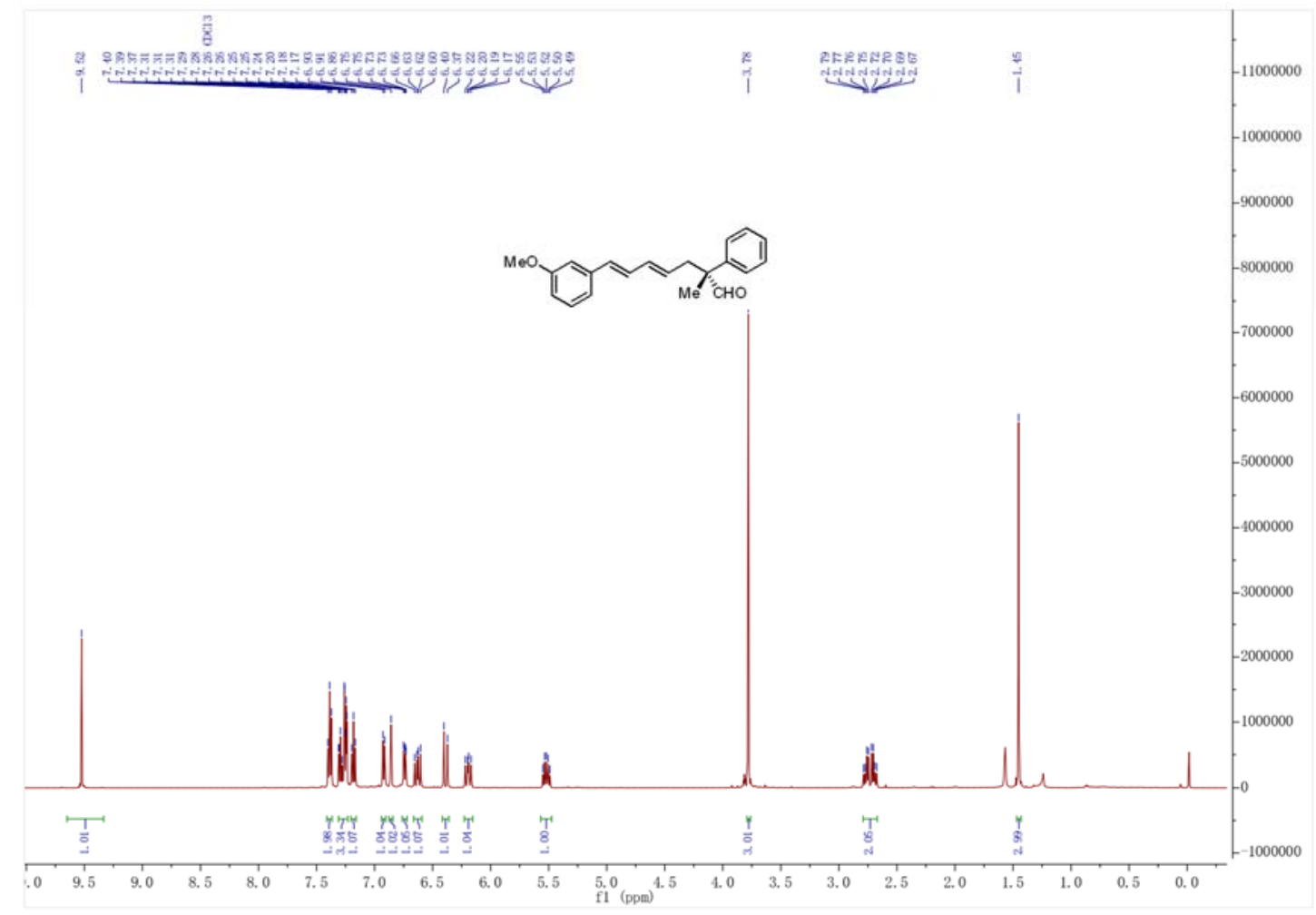


${ }^{13}$ C NMR (126 MHz, Chloroform-d)

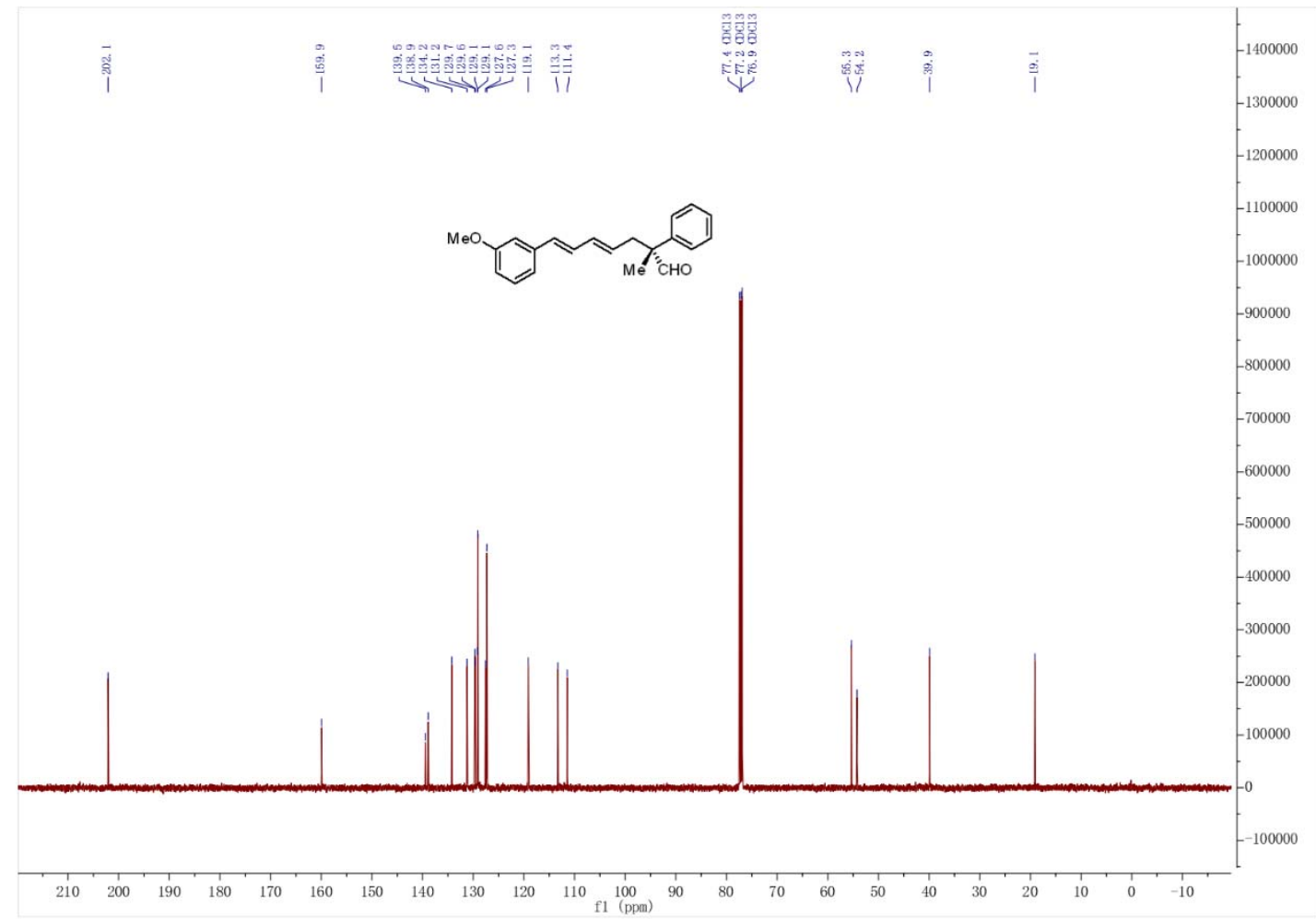

(R,4E,6E)-7-(2-fluorophenyl)-2-methyl-2-phenylhepta-4,6-dienal (3aa)

${ }^{1}$ H NMR (500 MHz, Chloroform-d)

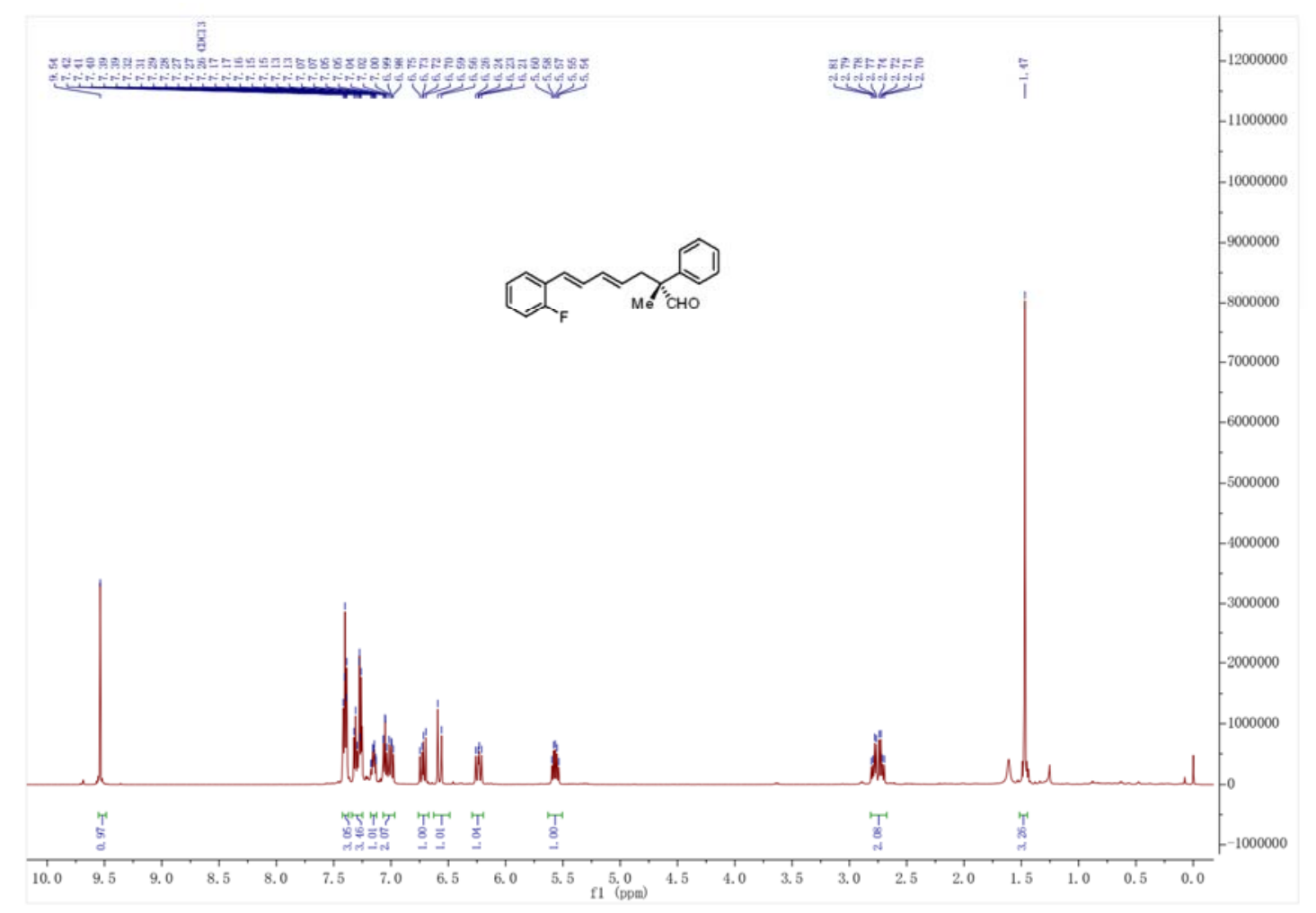


${ }^{13}$ C NMR (126 MHz, Chloroform-d)

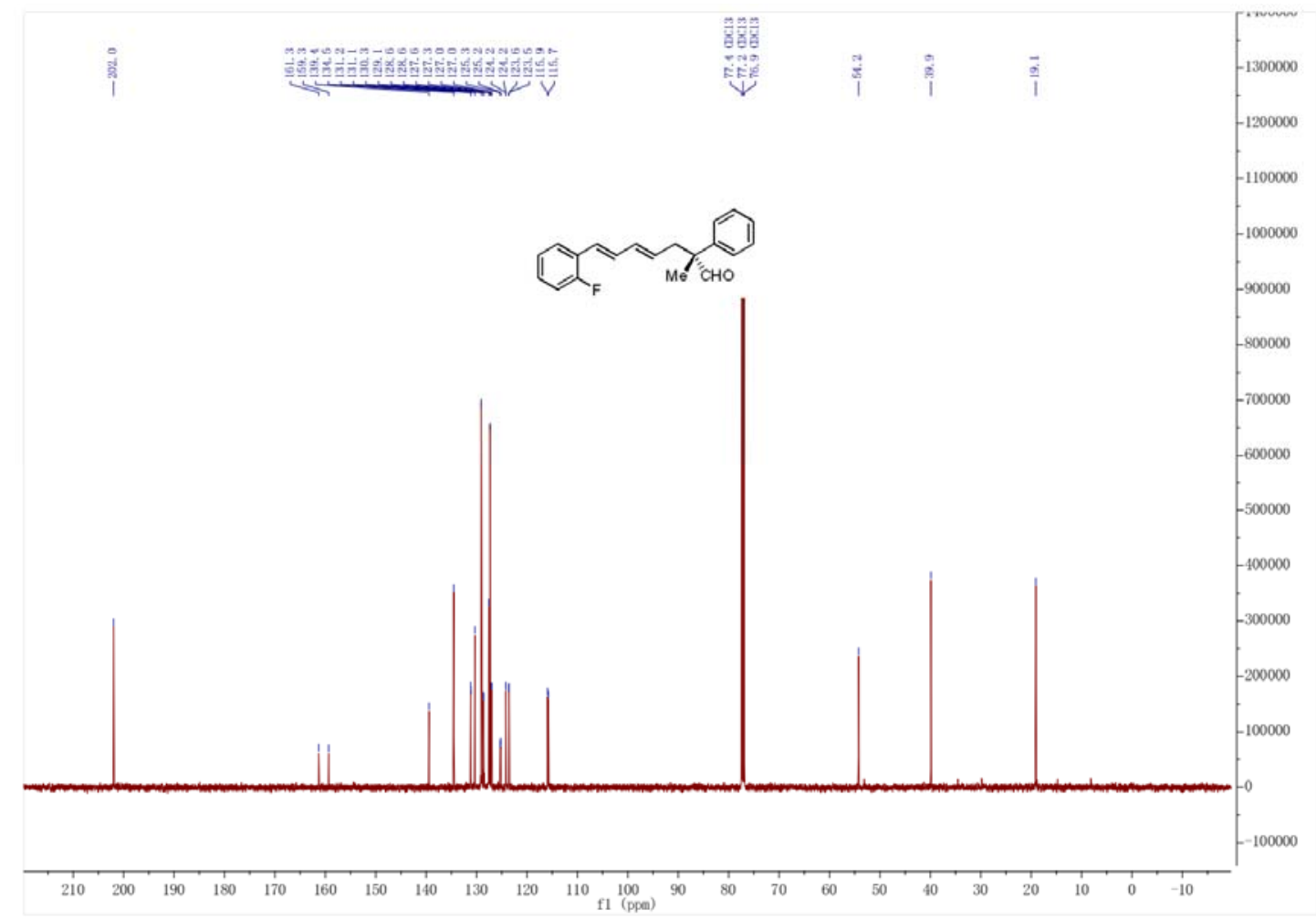

${ }^{19}$ F NMR (376 MHz, Chloroform-d)

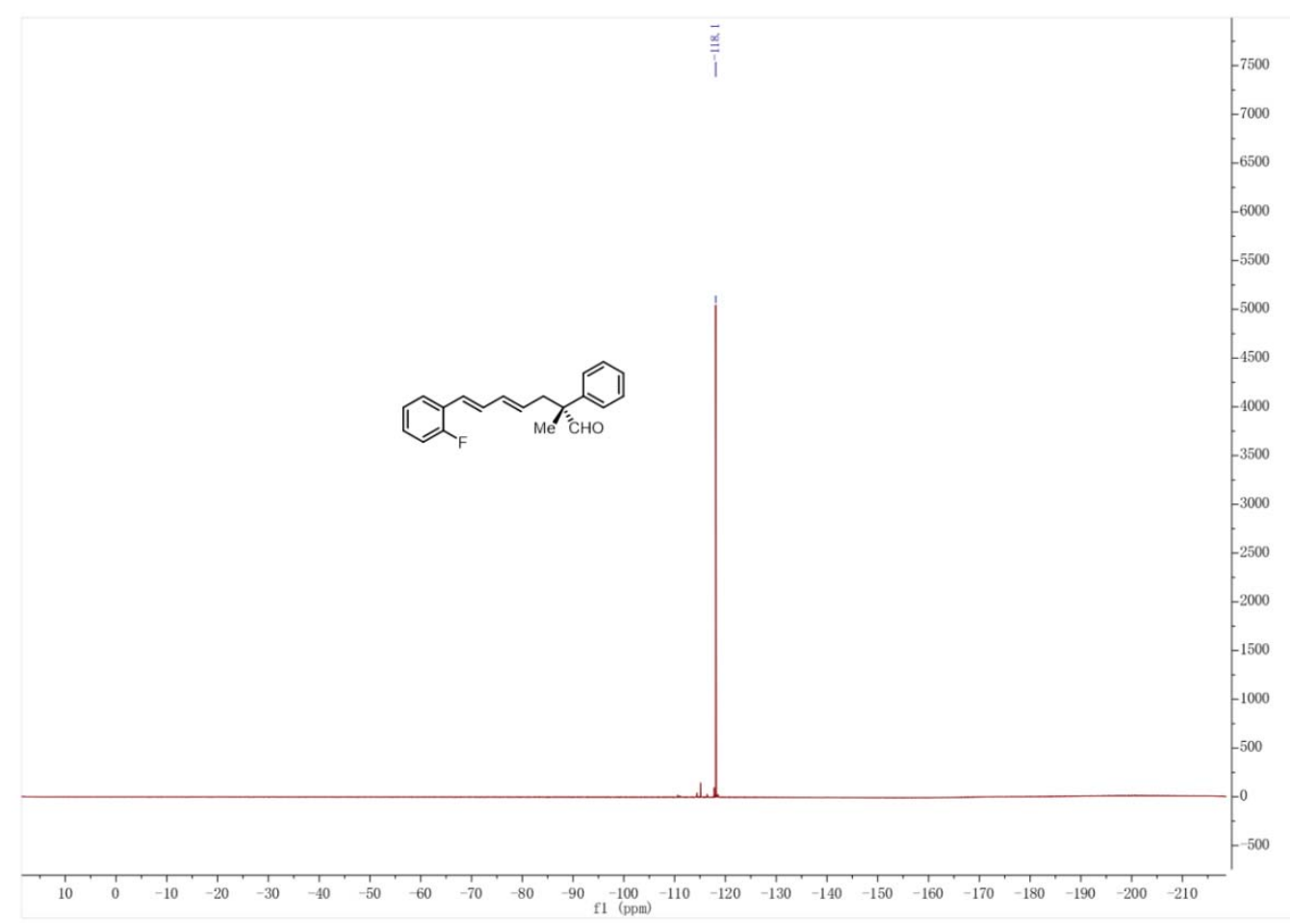


(R,4E,6E)-7-(2-methoxyphenyl)-2-methyl-2-phenylhepta-4,6-dienal (3ab)

${ }^{1}$ H NMR (400 MHz, Chloroform-d)

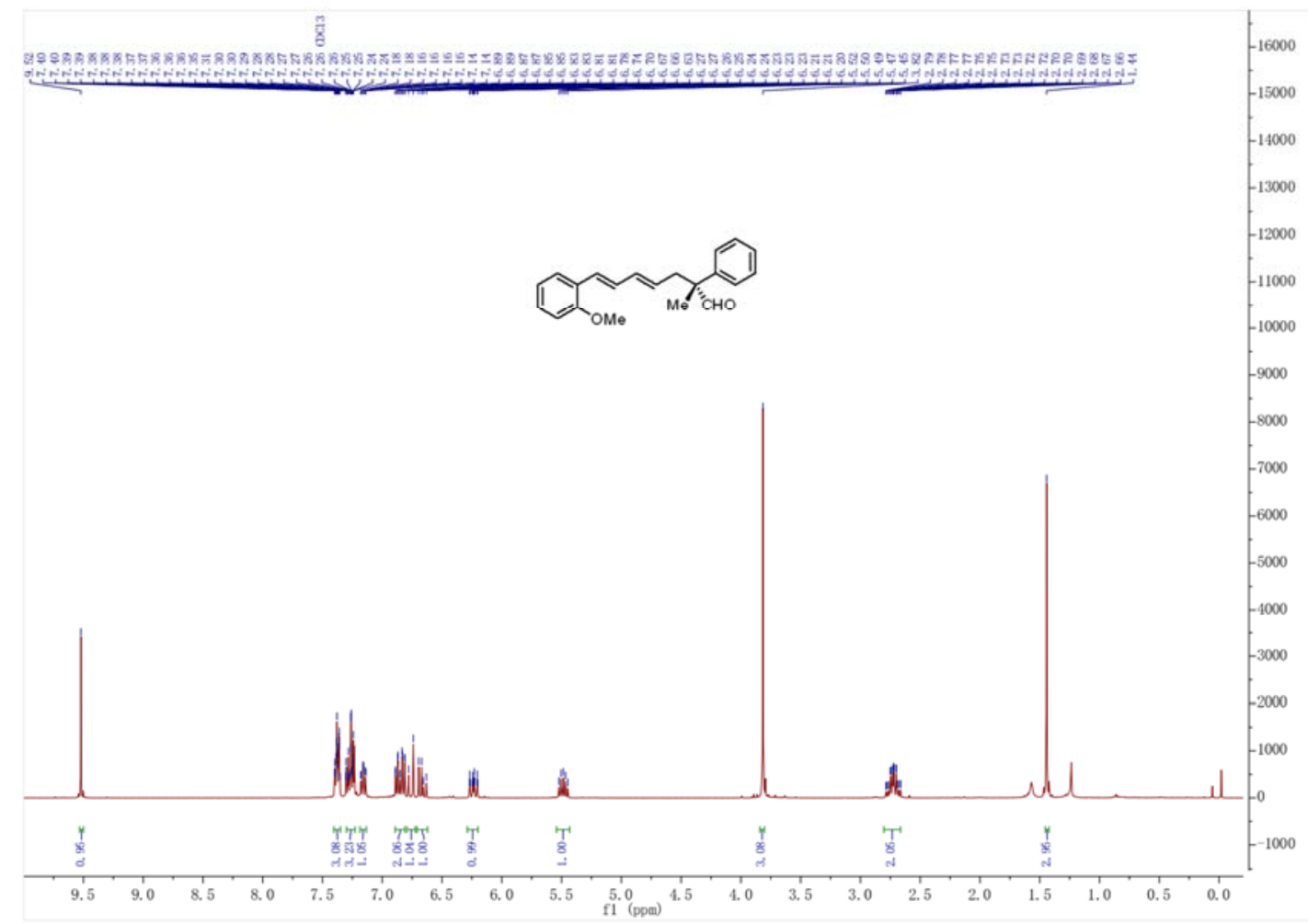

${ }^{13}$ C NMR (126 MHz, Chloroform-d)

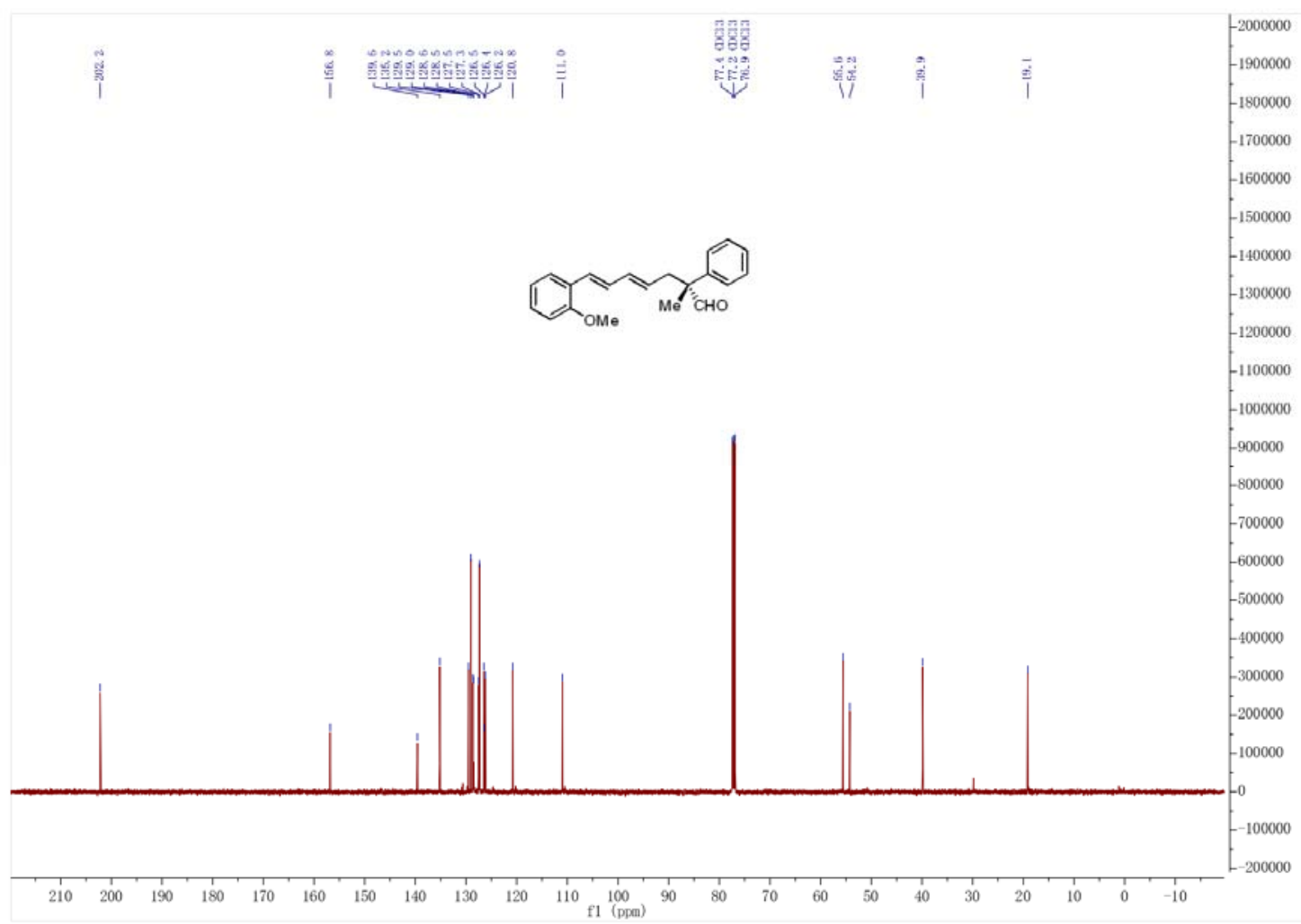


(R,4E,6E)-2-methyl-2-phenyl-7-(thiophen-3-yl)hepta-4,6-dienal (3ac)

${ }^{1}$ H NMR (500 MHz, Chloroform-d)

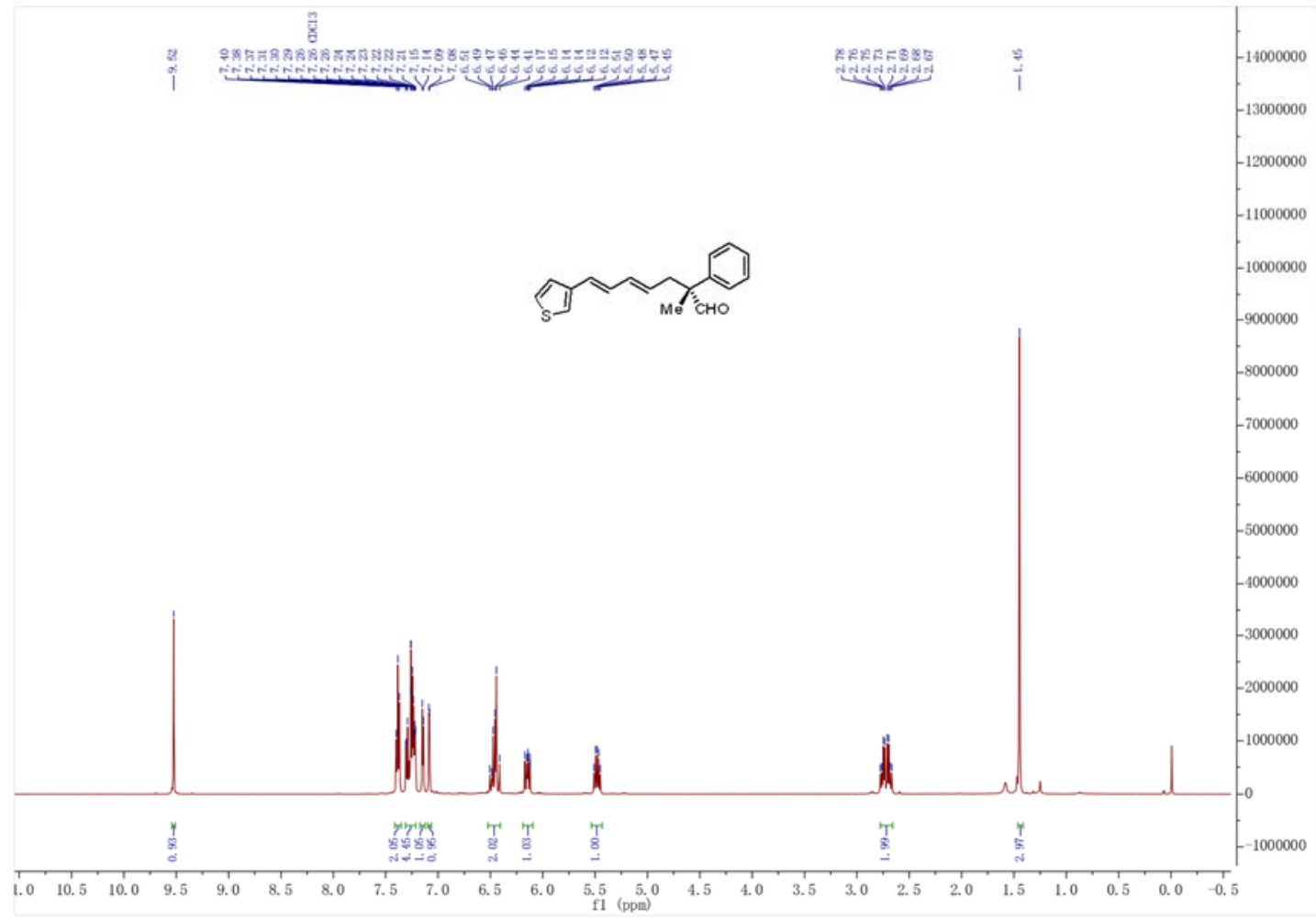

${ }^{13}$ C NMR (126 MHz, Chloroform-d)

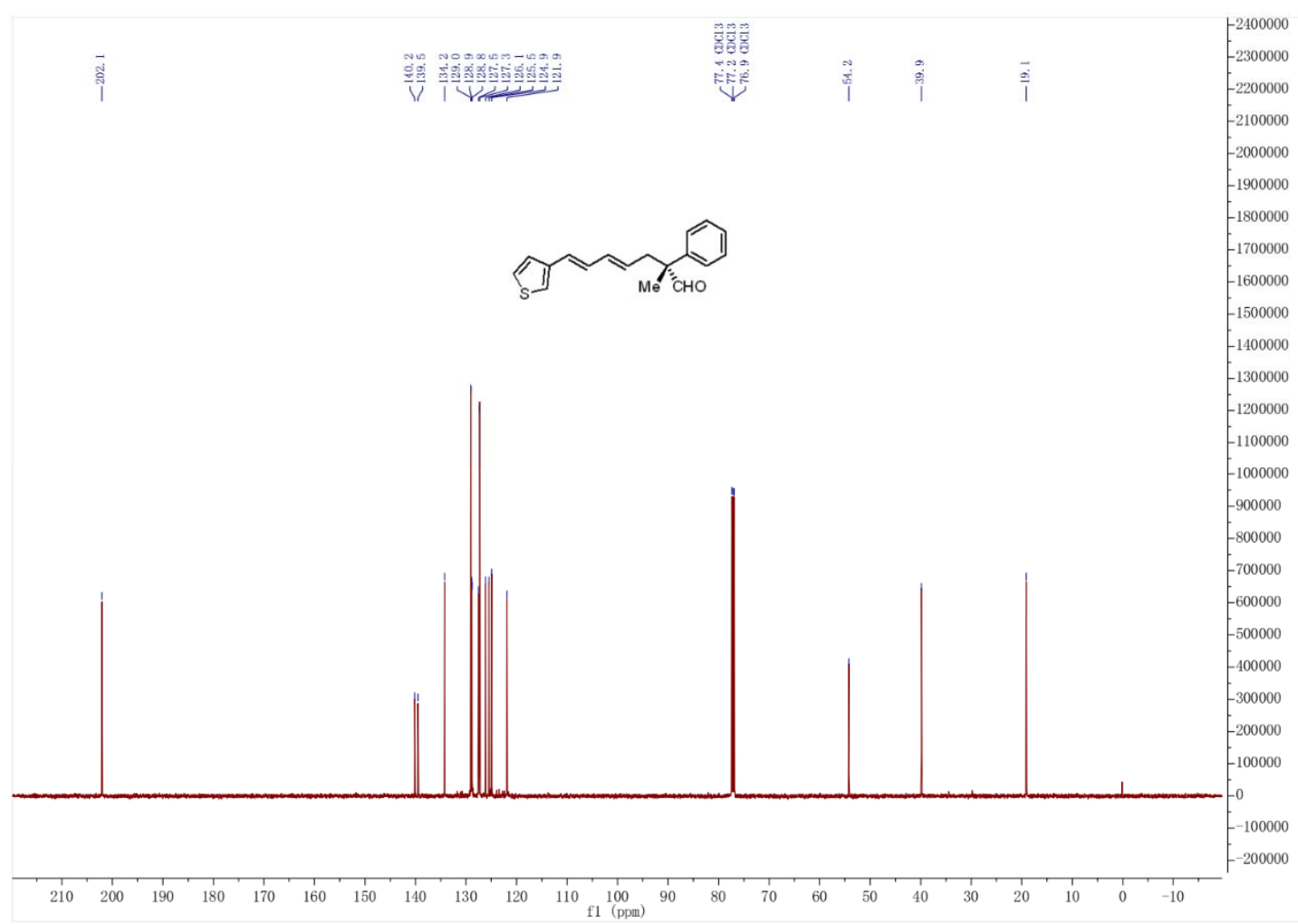


(2S,4R)-4-methyl-4-phenyl-2-((E)-prop-1-en-1-yl)tetrahydrofuran (4-major)

${ }^{1}$ H NMR (400 MHz, Chloroform-d)

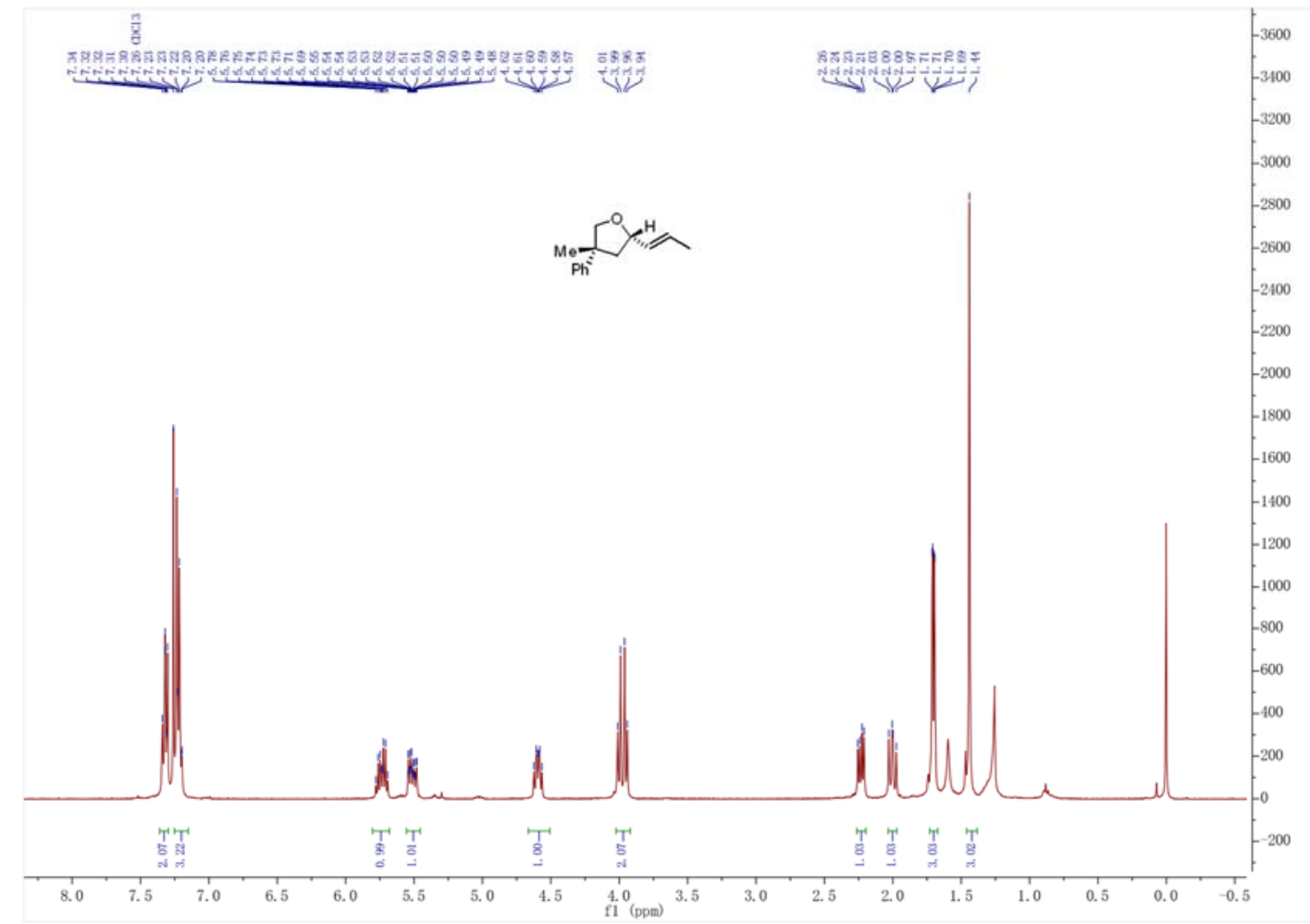

${ }^{13}$ C NMR (126 MHz, Chloroform-d)

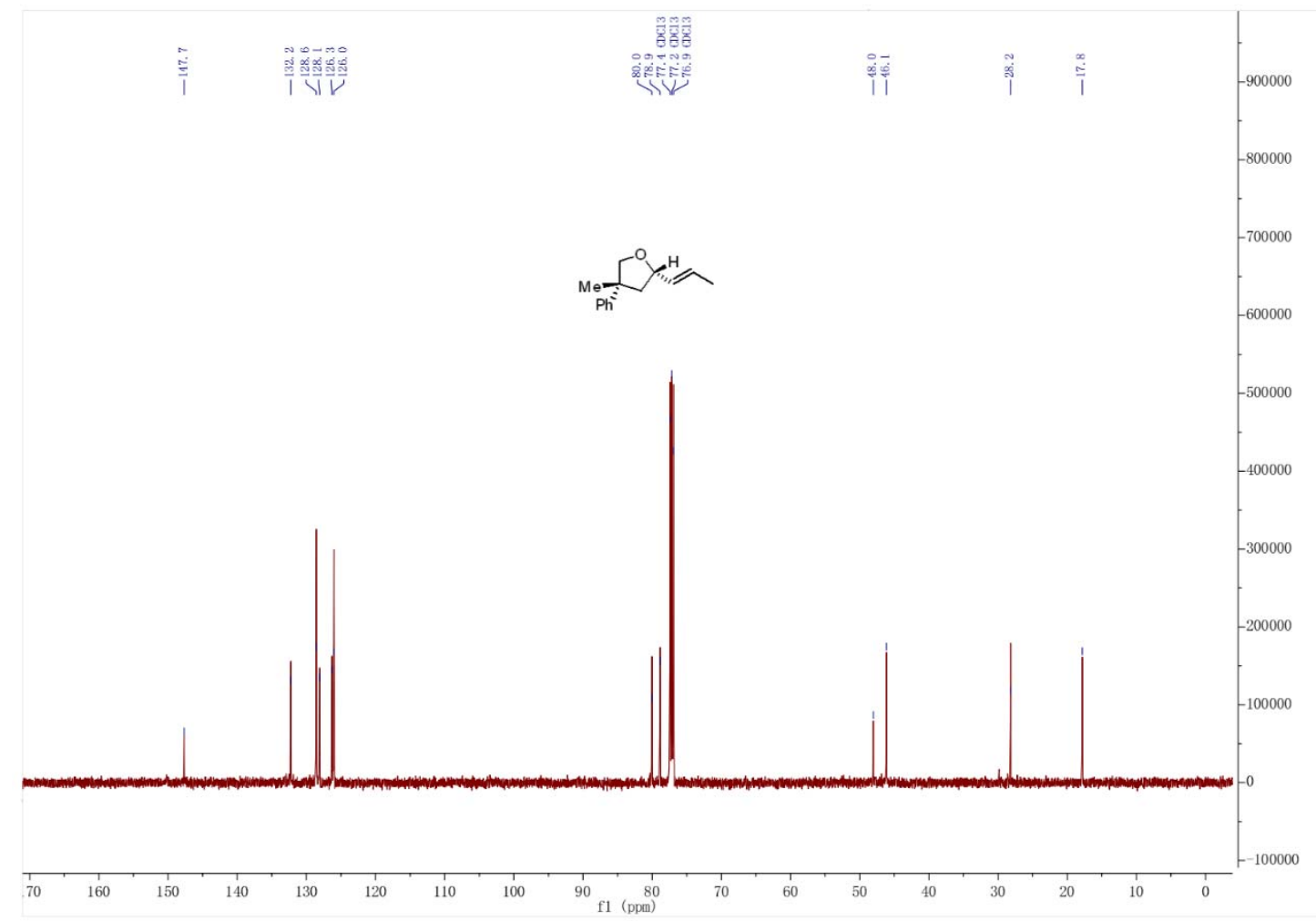


(2R,4R)-4-methyl-4-phenyl-2-((E)-prop-1-en-1-yl)tetrahydrofuran (4-minor)

${ }^{1}$ H NMR (400 MHz, Chloroform-d)

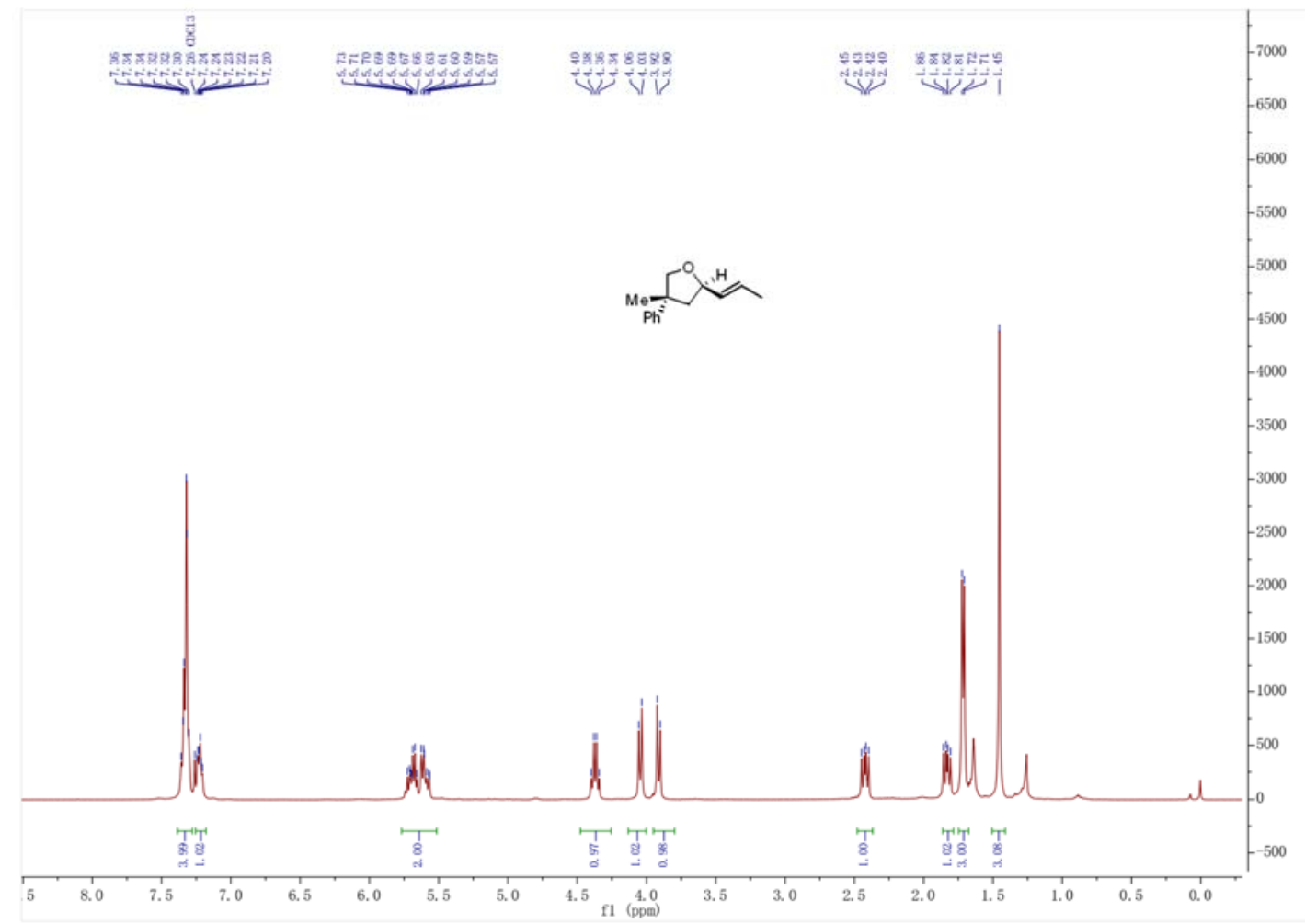

${ }^{13}$ C NMR (126 MHz, Chloroform-d)

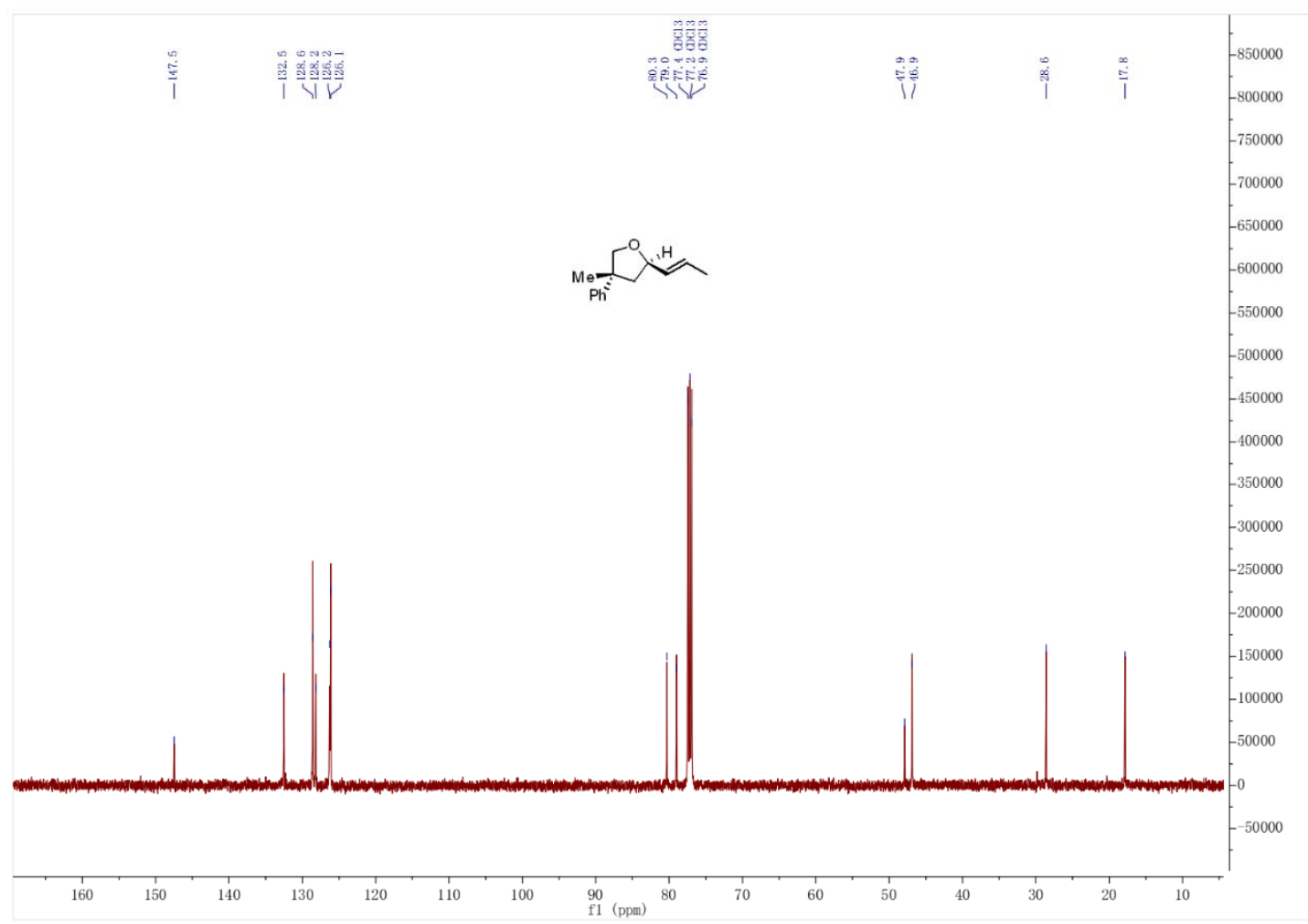


(R,E)-N-benzyl-2-methyl-2-phenylhepta-4,6-dien-1-amine (5)

${ }^{1}$ H NMR (400 MHz, Chloroform-d)

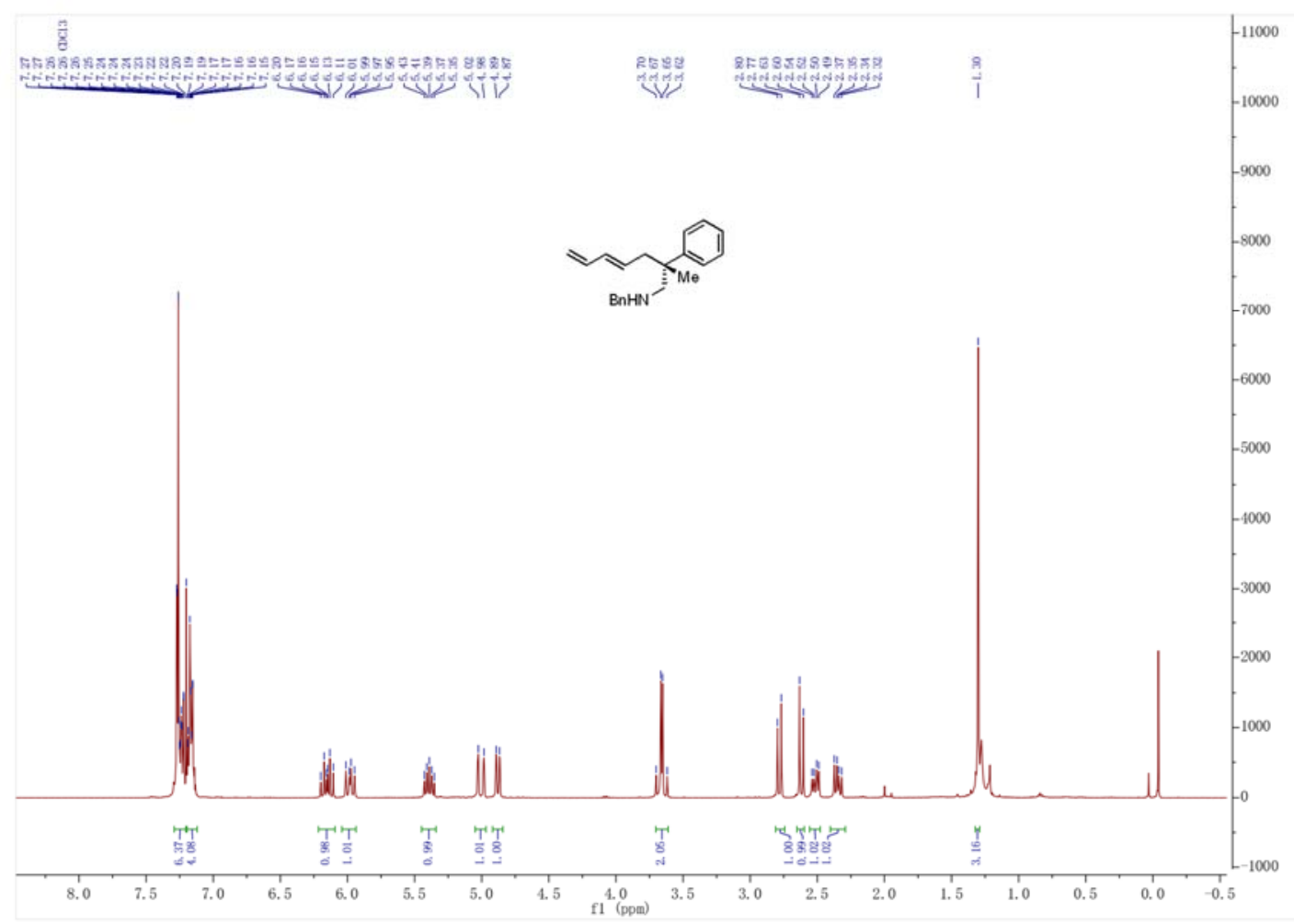

${ }^{13}$ C NMR (126 MHz, Chloroform-d)

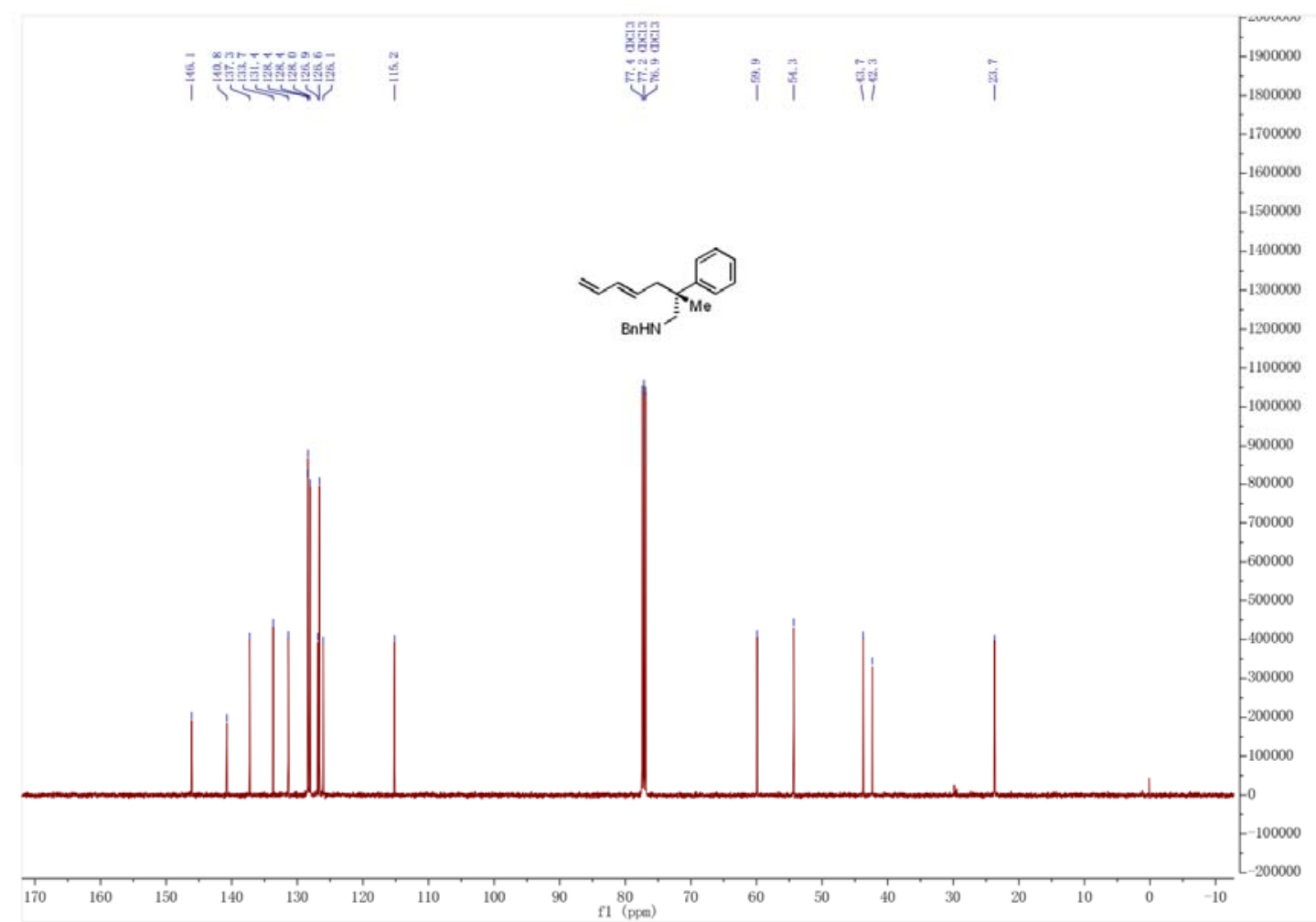


(S,E)-(3-methylocta-5,7-dien-1-yn-3-yl)benzene (6)

${ }^{1}$ H NMR (400 MHz, Chloroform-d)

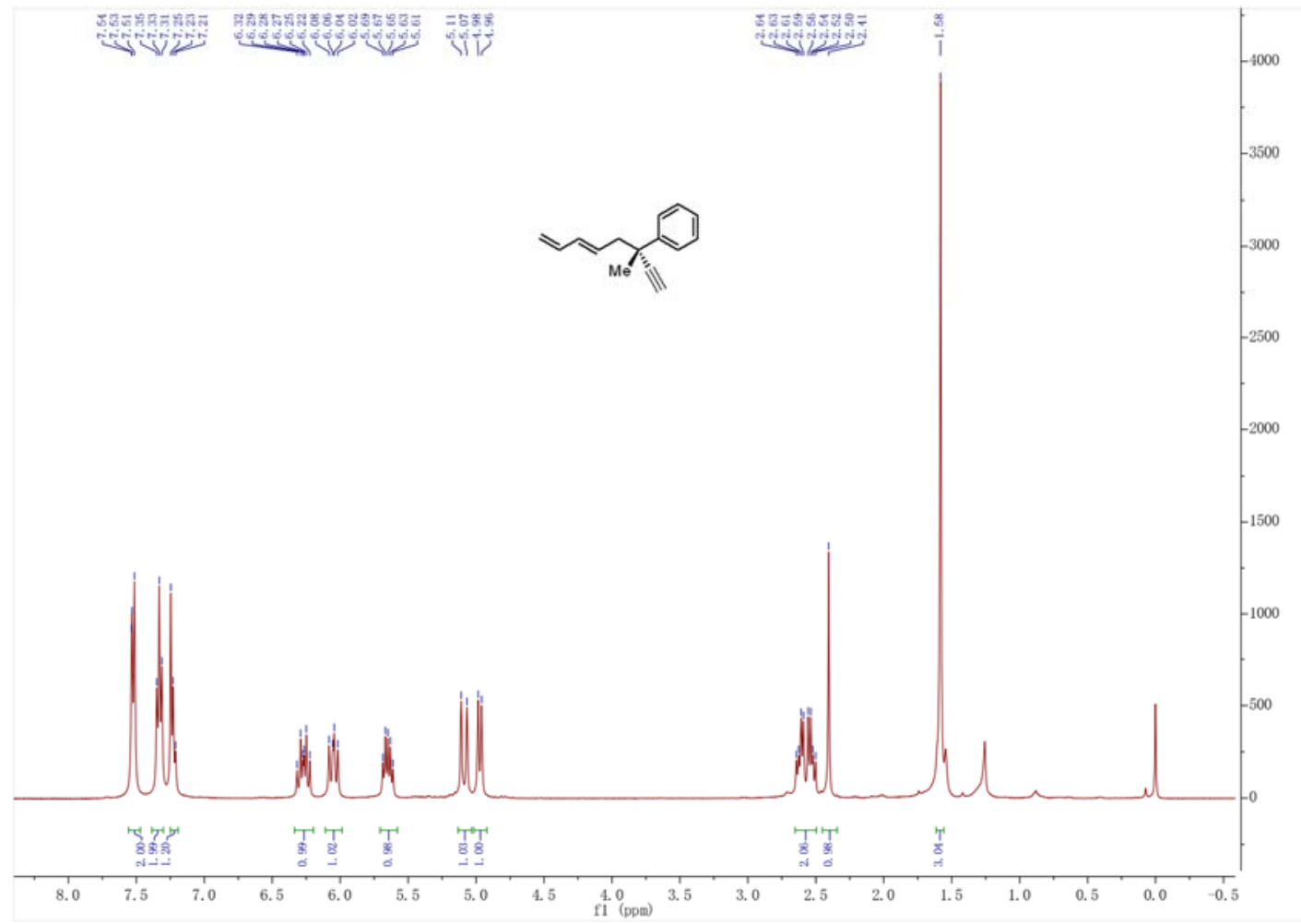

${ }^{13}$ C NMR (101 MHz, Chloroform-d)

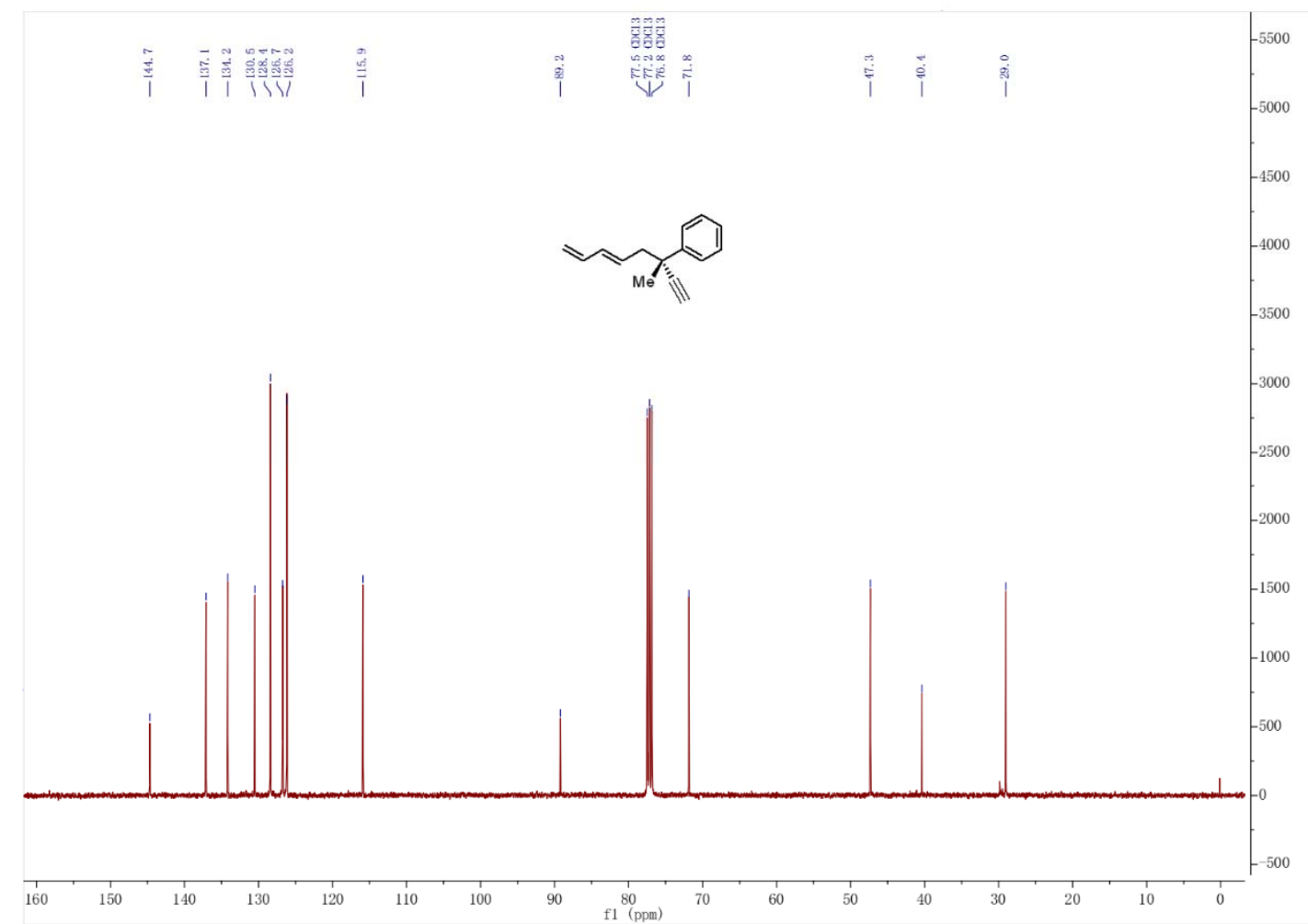




\section{HPLC data}

\section{(R,E)-2-methyl-2-phenylhepta-4,6-dienal (3a)}

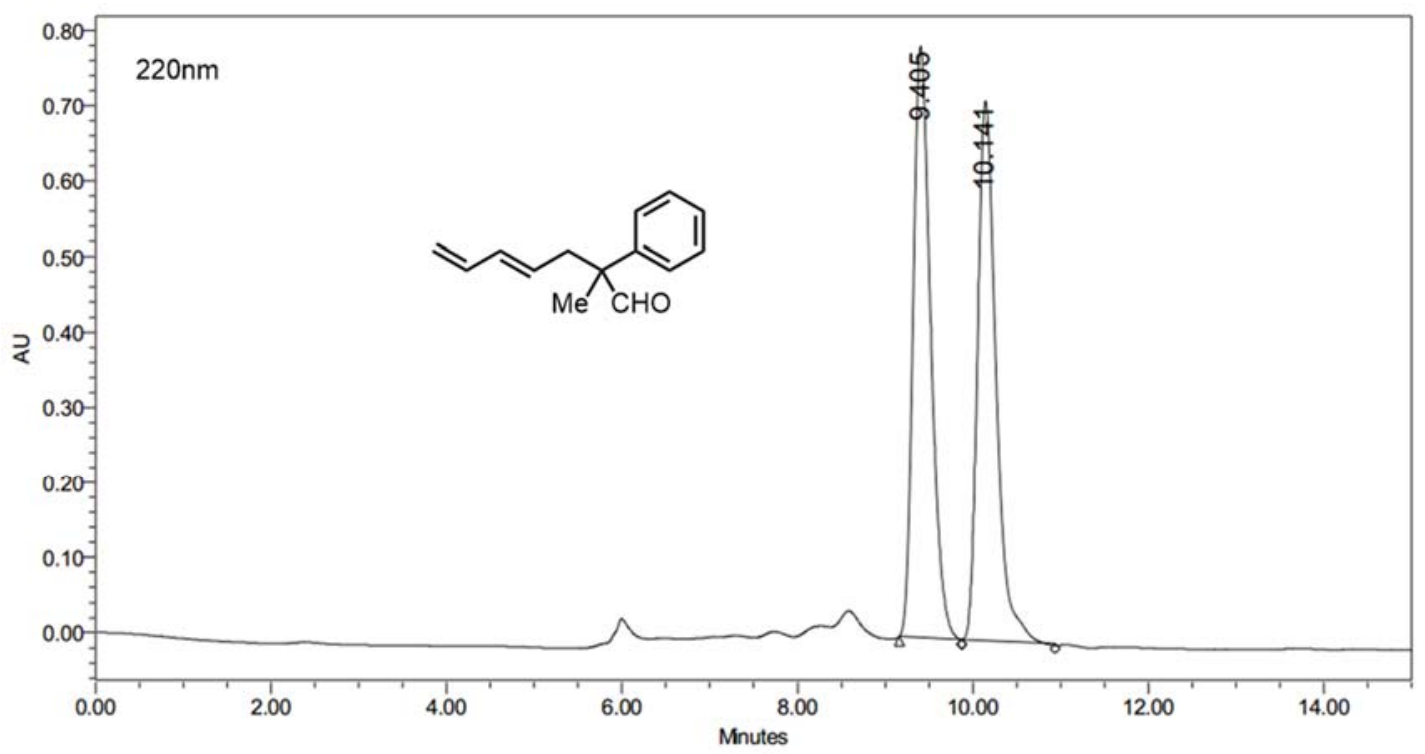

\begin{tabular}{|l|r|c|r|c|}
\hline & \multicolumn{1}{|c|}{ RT } & Area & $\%$ Area & Height \\
\hline 1 & 9.405 & 10903182 & 51.14 & 784854 \\
\hline 2 & 10.141 & 10415080 & 48.86 & 716252 \\
\hline
\end{tabular}

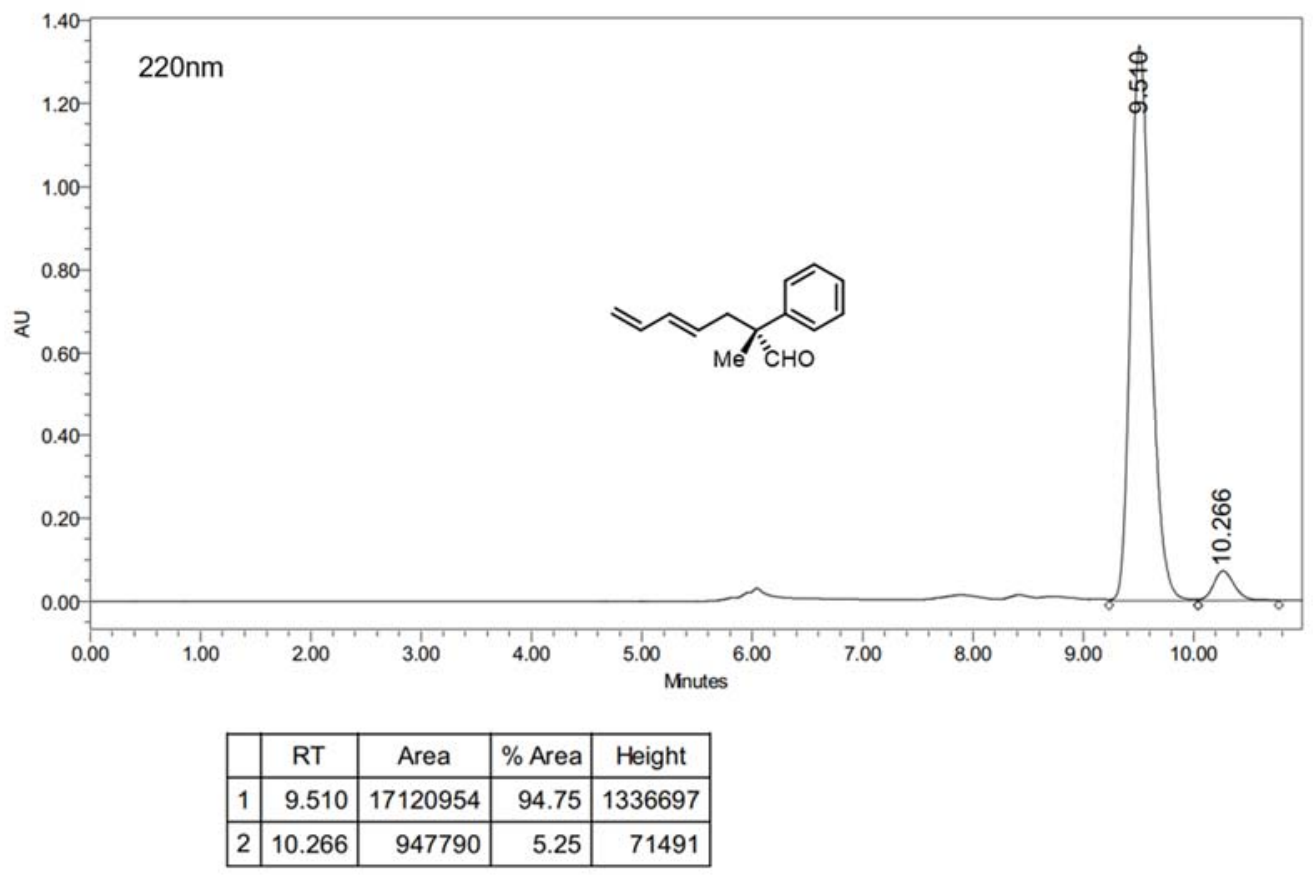


(R,E)-2-methyl-2-(p-tolyl)hepta-4,6-dienal (3b)

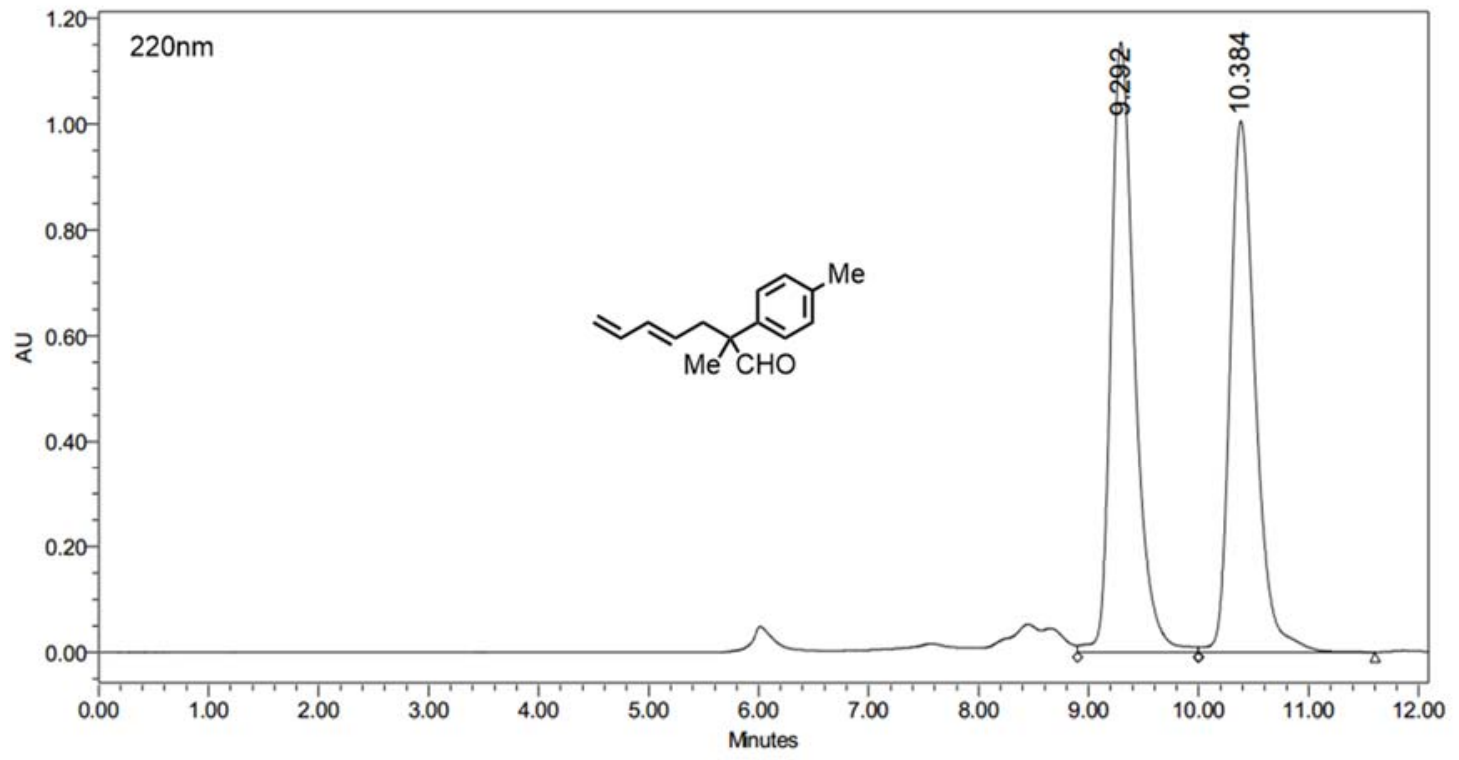

\begin{tabular}{|c|c|c|r|c|}
\hline & \multicolumn{1}{|c|}{ RT } & Area & \% Area & Height \\
\hline 1 & 9.292 & 17307855 & 51.82 & 1154053 \\
\hline 2 & 10.384 & 16093937 & 48.18 & 1005831 \\
\hline
\end{tabular}

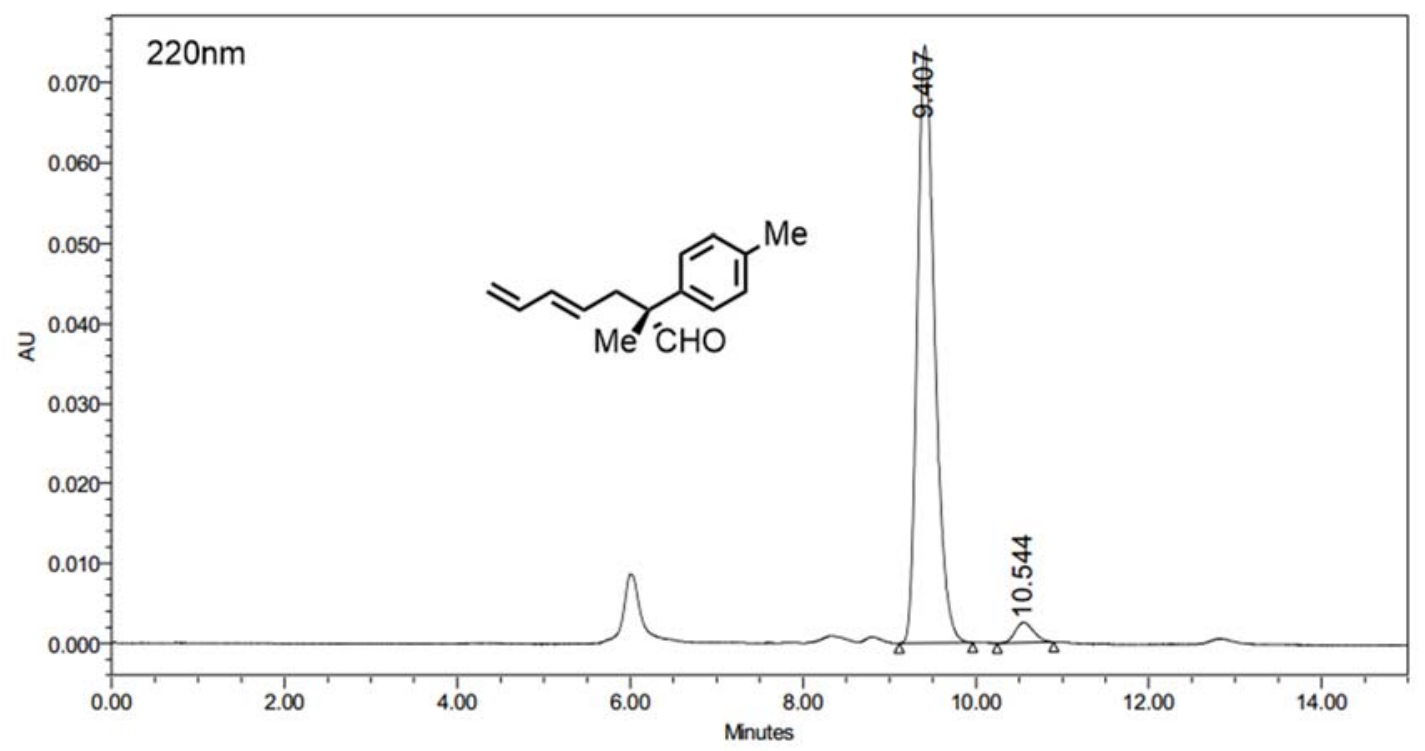

\begin{tabular}{|l|c|r|r|r|}
\hline & \multicolumn{1}{|c|}{ RT } & \multicolumn{1}{c|}{ Area } & \% Area & Height \\
\hline 1 & 9.407 & 1044570 & 96.63 & 74603 \\
\hline 2 & 10.544 & 36417 & 3.37 & 2483 \\
\hline
\end{tabular}


(R,E)-2-(4-methoxyphenyl)-2-me thylhepta-4,6-dienal (3c)

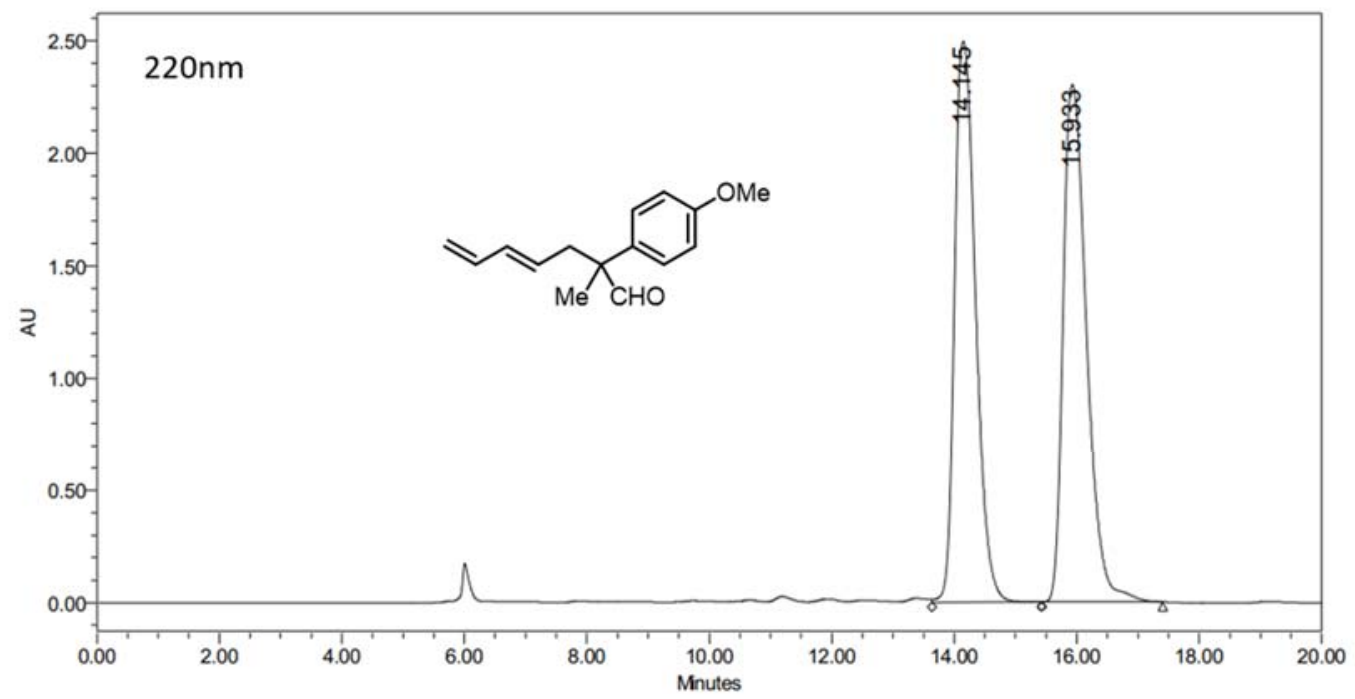

\begin{tabular}{|c|c|c|r|c|}
\hline & RT & Area & $\%$ Area & Height \\
\hline 1 & 14.145 & 59423723 & 49.24 & 2493419 \\
\hline 2 & 15.933 & 61250951 & 50.76 & 2301456 \\
\hline
\end{tabular}

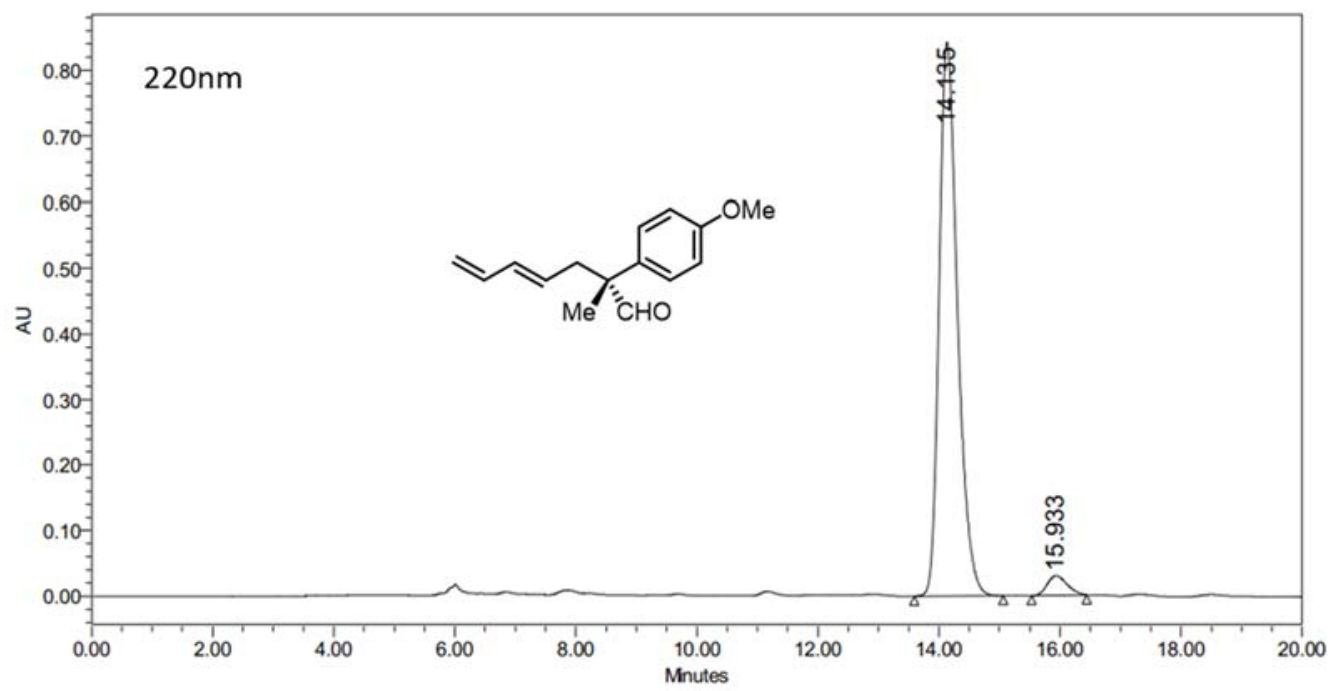

\begin{tabular}{|r|c|r|r|r|}
\hline & RT & \multicolumn{1}{c|}{ Area } & $\%$ Area & Height \\
\hline 1 & 14.135 & 17767523 & 96.33 & 841868 \\
\hline 2 & 15.933 & 676274 & 3.67 & 29780 \\
\hline
\end{tabular}


(R,E)-2-(4-fluorophenyl)-2-methylhepta-4,6-dienal (3d)

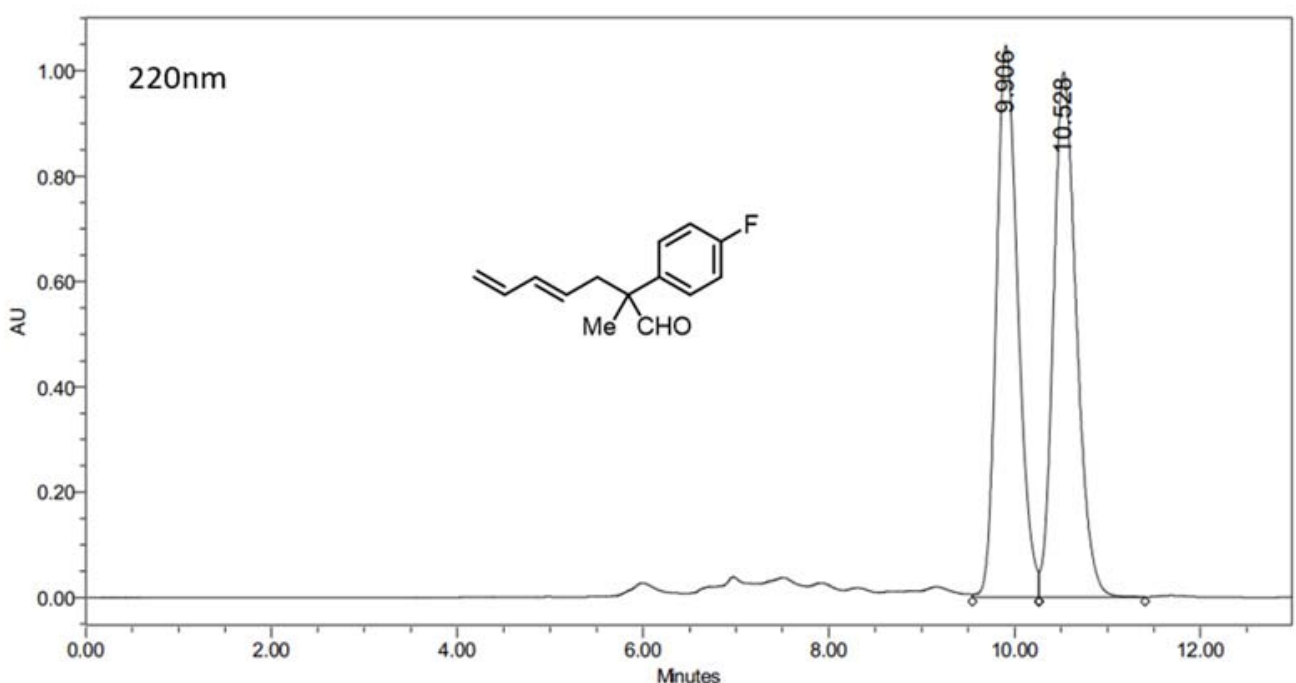

\begin{tabular}{|l|r|c|r|r|}
\hline & \multicolumn{1}{|c|}{ RT } & Area & $\%$ Area & \multicolumn{1}{c|}{ Height } \\
\hline 1 & 9.906 & 16868055 & 49.74 & 1046730 \\
\hline 2 & 10.528 & 17046903 & 50.26 & 995675 \\
\hline
\end{tabular}

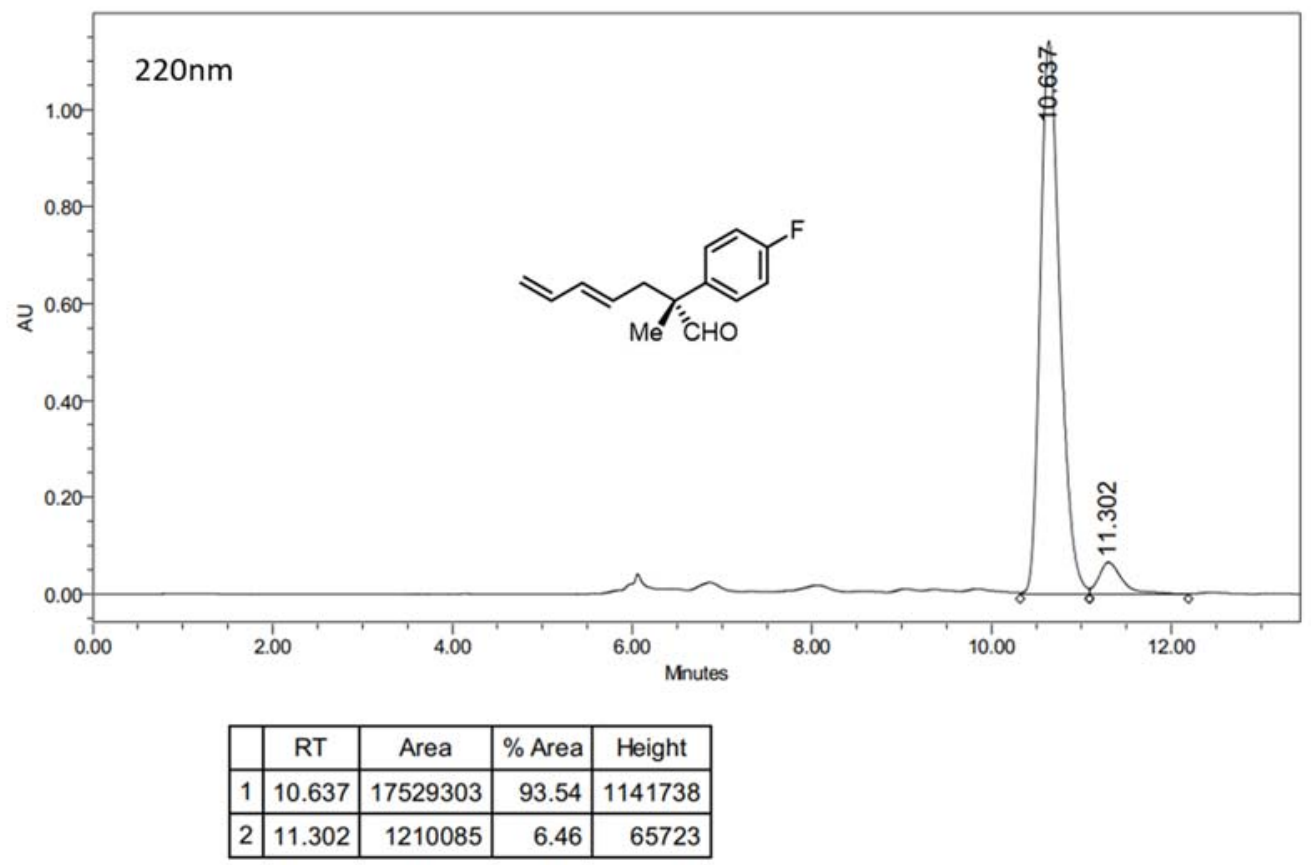


(R,E)-2-(3-chlorophenyl)-2-methylhepta-4,6-dienal (3e)

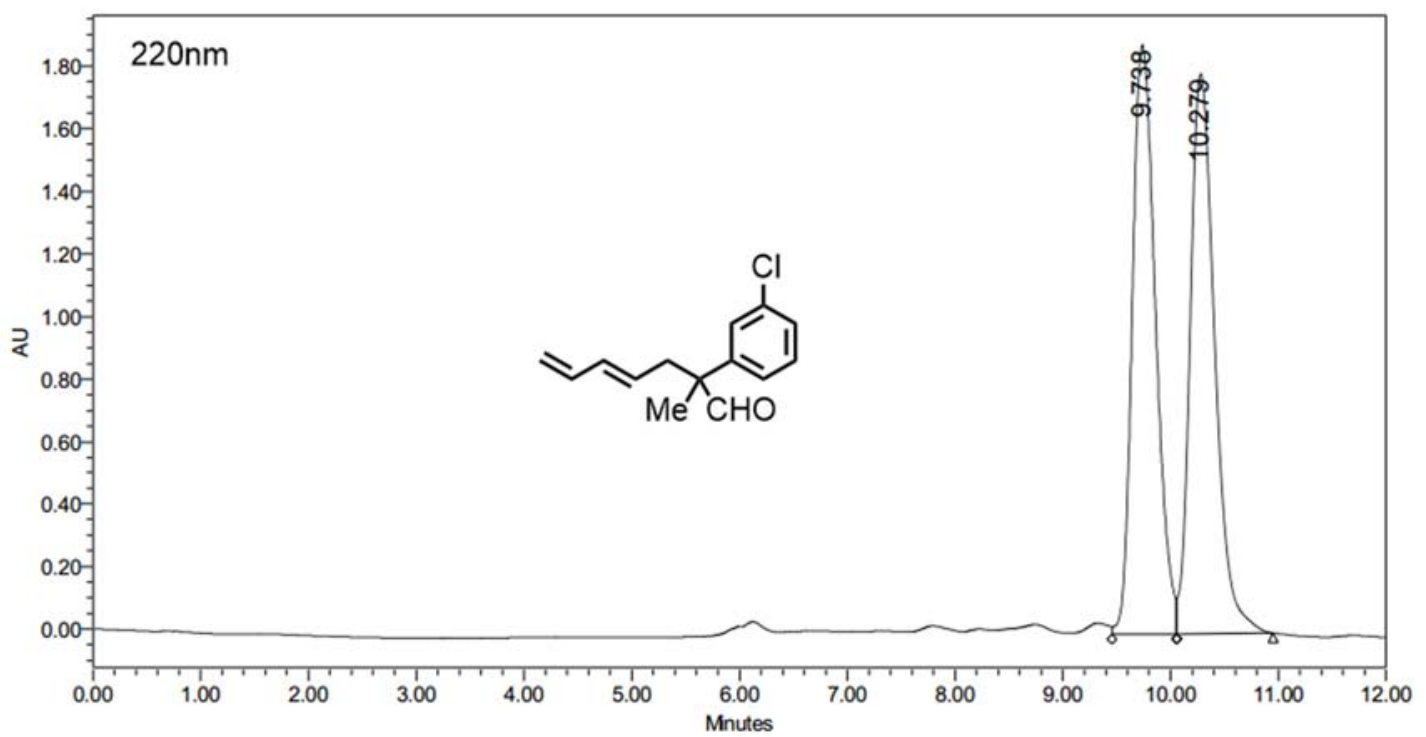

\begin{tabular}{|c|c|c|r|c|}
\hline & \multicolumn{1}{|c|}{ RT } & Area & $\%$ Area & Height \\
\hline 1 & 9.738 & 28147162 & 49.70 & 1882299 \\
\hline 2 & 10.279 & 28481817 & 50.30 & 1789058 \\
\hline
\end{tabular}

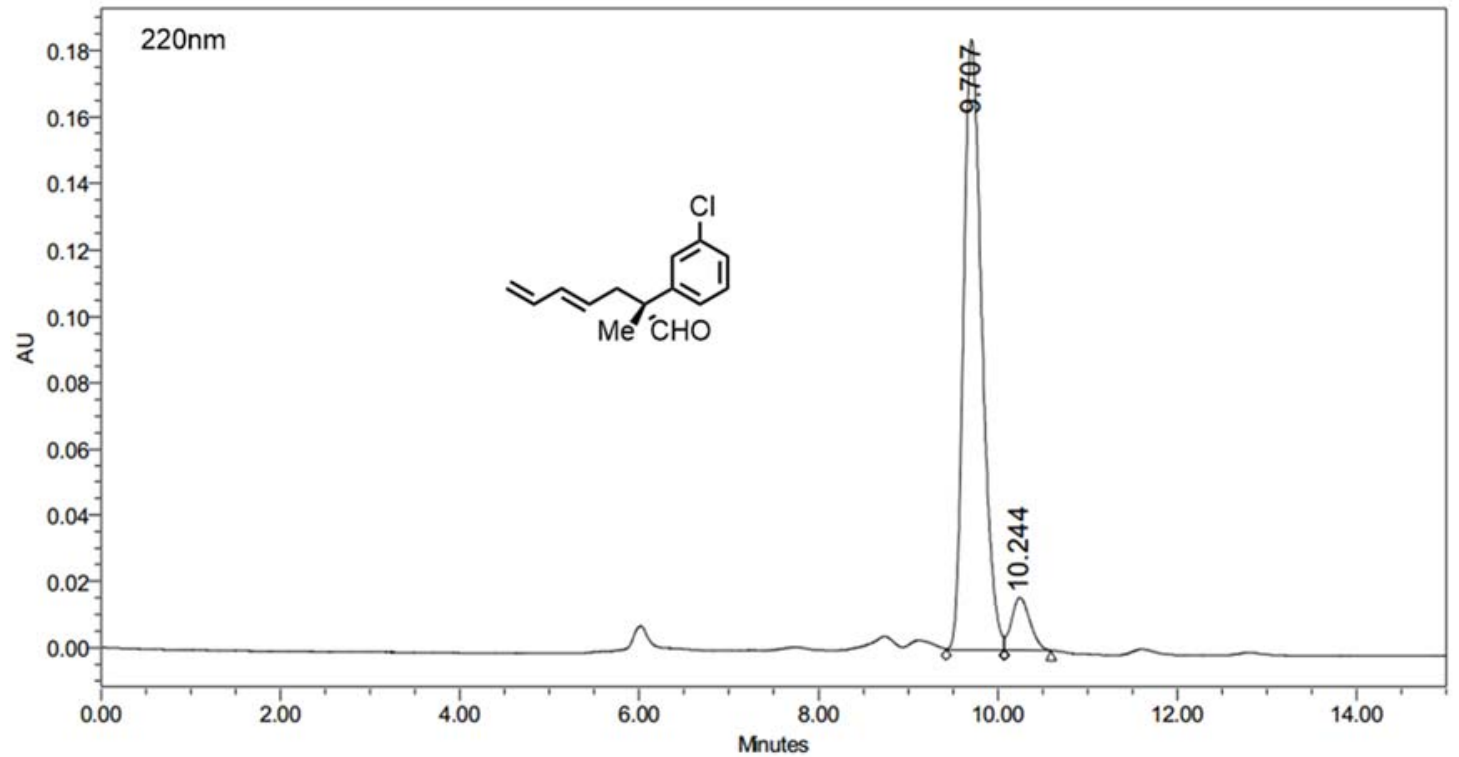

\begin{tabular}{|l|c|r|r|r|}
\hline & \multicolumn{1}{|c|}{ RT } & \multicolumn{1}{c|}{ Area } & \% Area & Height \\
\hline 1 & 9.707 & 2607873 & 91.97 & 184143 \\
\hline 2 & 10.244 & 227850 & 8.03 & 15923 \\
\hline
\end{tabular}


(R,E)-2-methyl-2-(m-tolyl)hepta-4,6-dienal (3f)

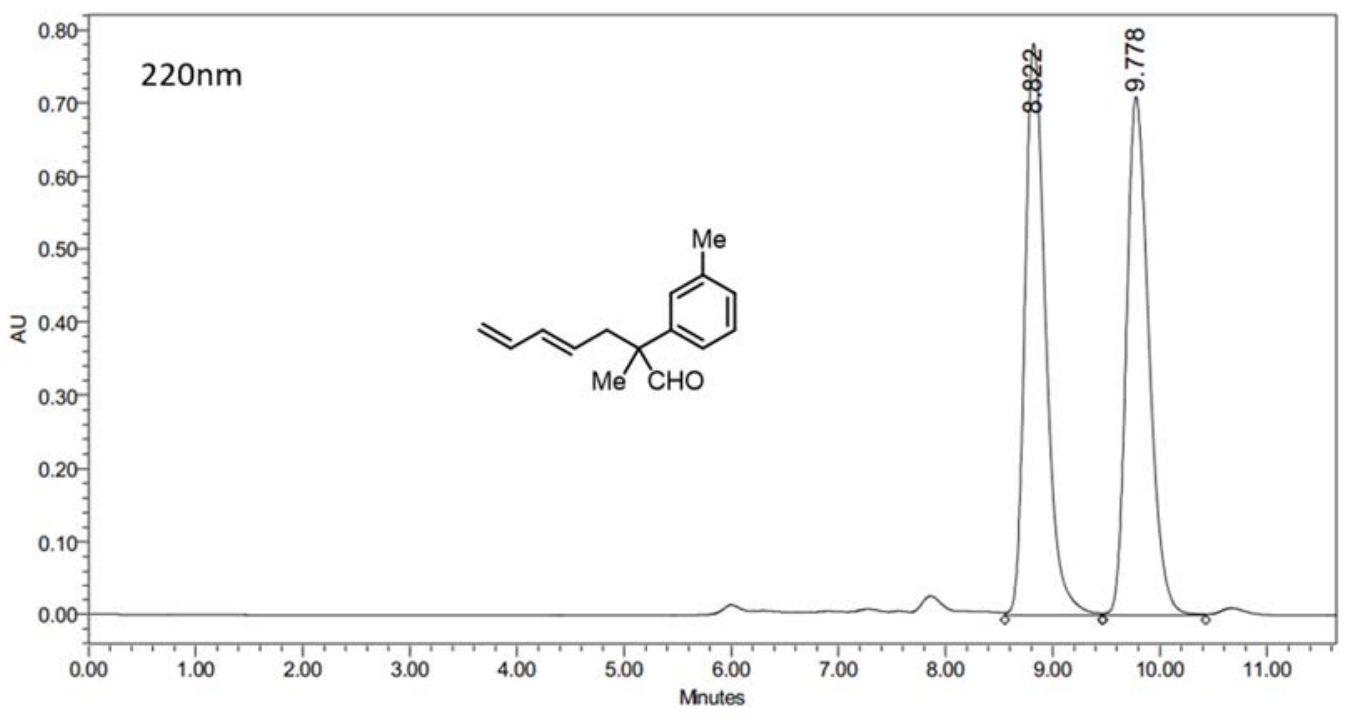

\begin{tabular}{|c|c|c|r|c|}
\hline & RT & Area & \% Area & Height \\
\hline 1 & 8.822 & 10551050 & 50.51 & 782723 \\
\hline 2 & 9.778 & 10339811 & 49.49 & 709863 \\
\hline
\end{tabular}

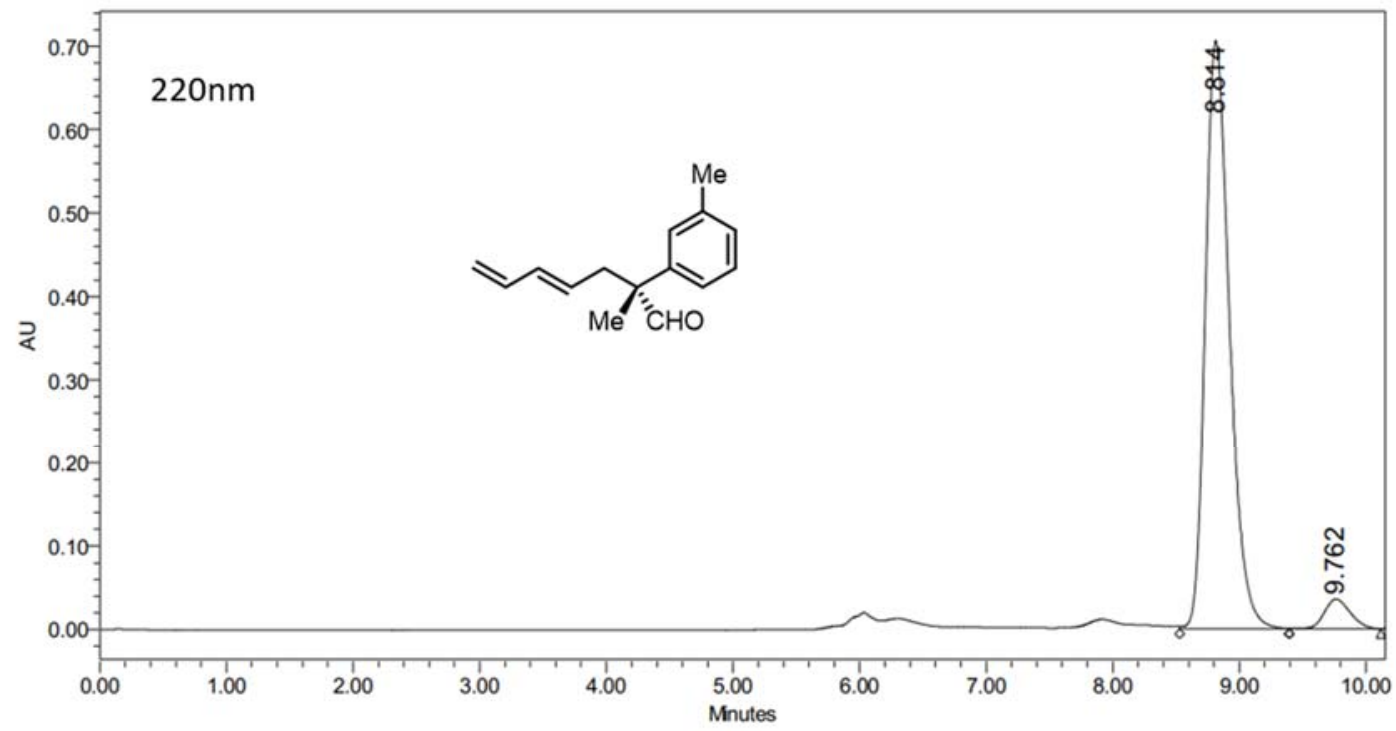

\begin{tabular}{|r|c|c|r|r|}
\hline & RT & \multicolumn{1}{c|}{ Area } & \% Area & Height \\
\hline 1 & 8.814 & 9395172 & 94.81 & 705971 \\
\hline 2 & 9.762 & 514171 & 5.19 & 35569 \\
\hline
\end{tabular}


(R,E)-2-(2-methoxyphenyl)-2-methylhepta-4,6-dienal (3g)
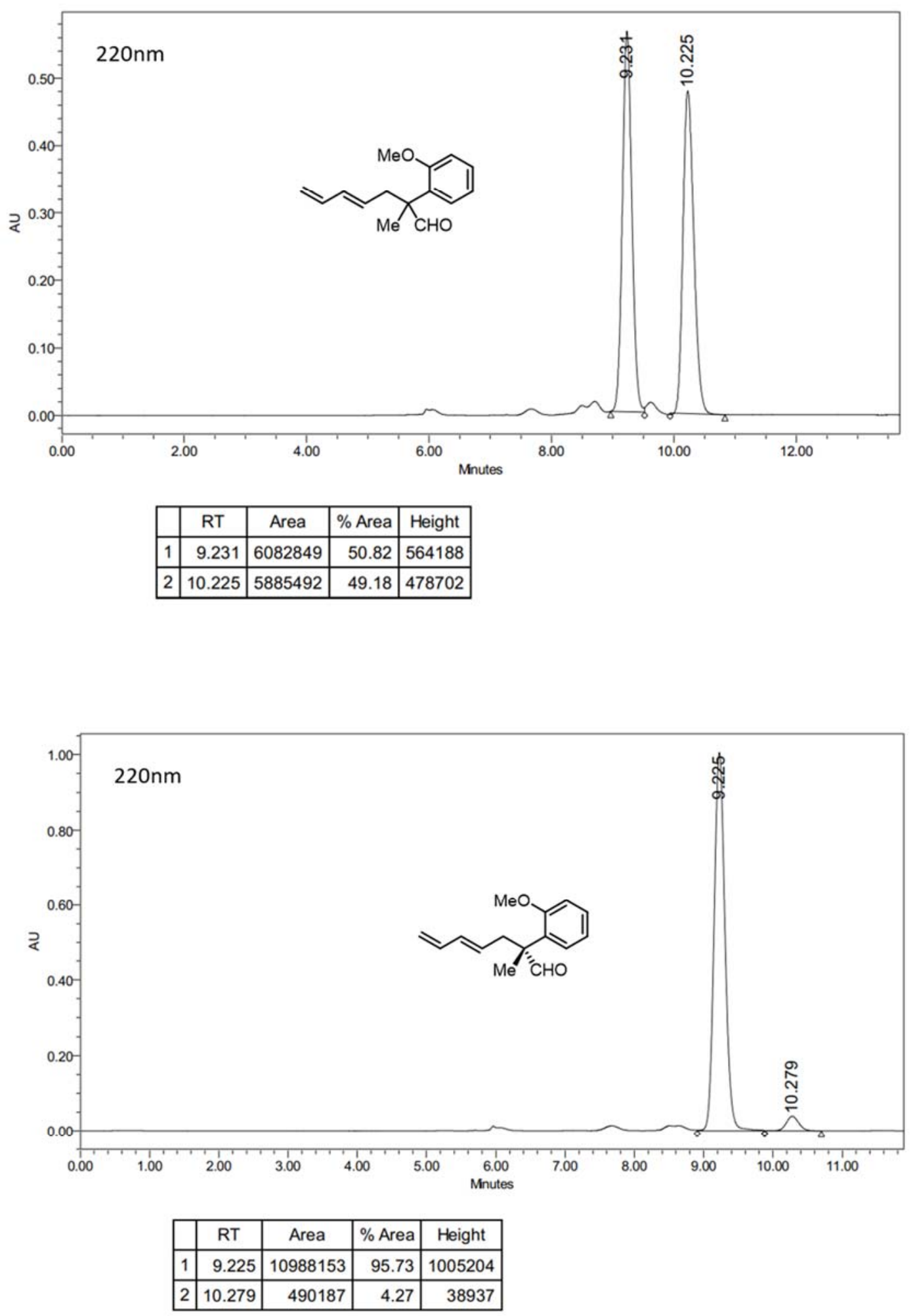
(R,E)-2-(2-fluorophenyl)-2-methylhepta-4,6-dienal (3h)

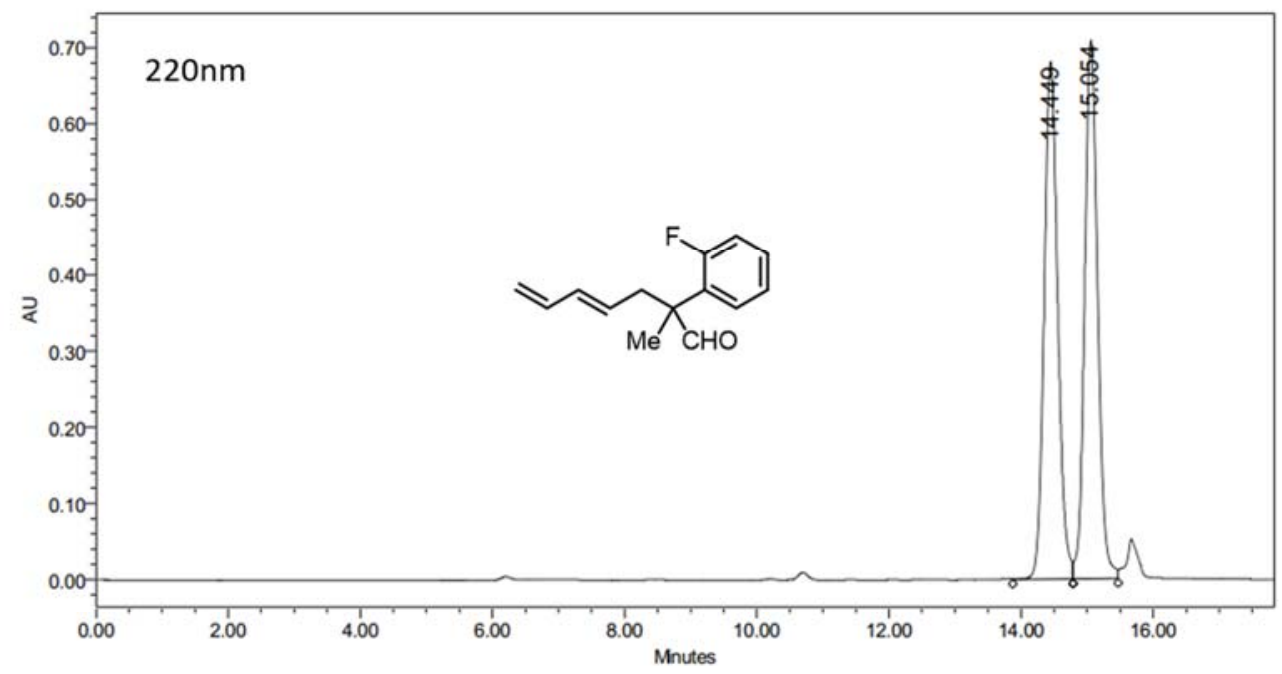

\begin{tabular}{|c|c|c|r|c|}
\hline & RT & Area & $\%$ Area & Height \\
\hline 1 & 14.449 & 9709030 & 50.04 & 680640 \\
\hline 2 & 15.054 & 9691893 & 49.96 & 708421 \\
\hline
\end{tabular}

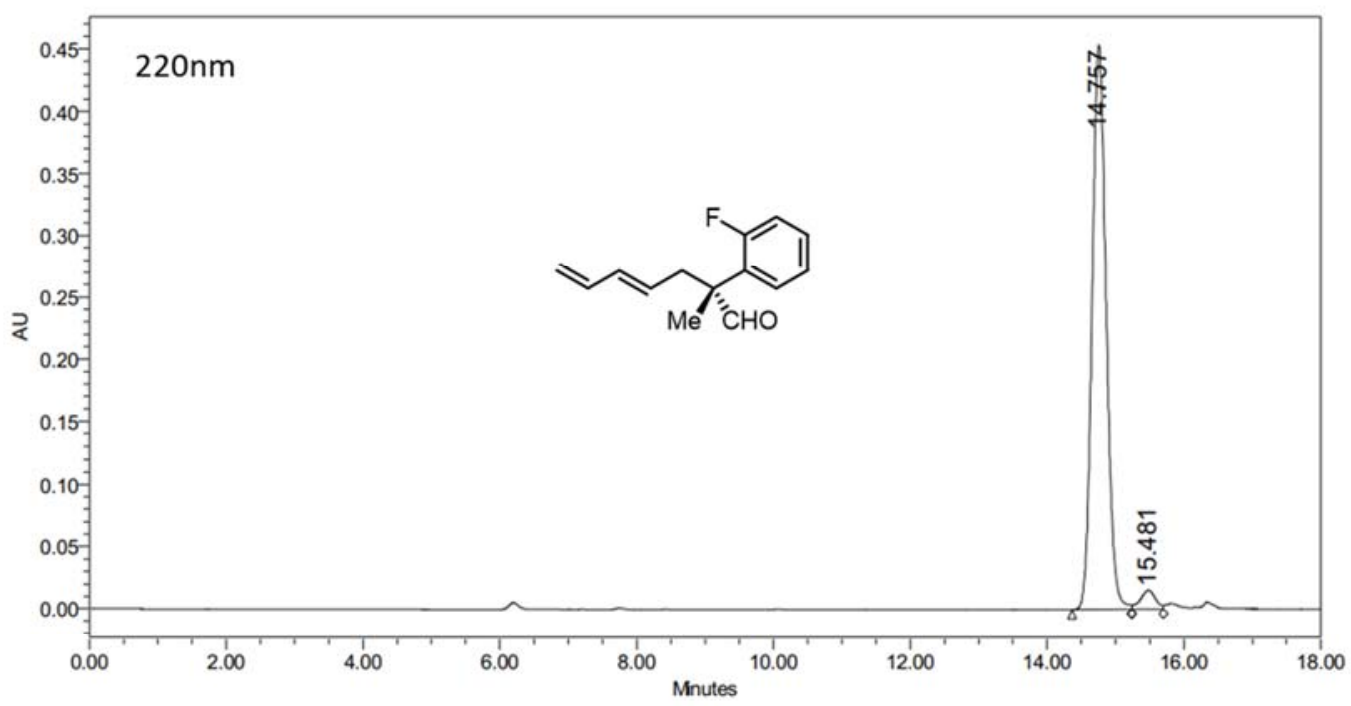

\begin{tabular}{|r|c|r|r|r|}
\hline & RT & Area & $\%$ Area & Height \\
\hline 1 & 14.757 & 6427225 & 96.52 & 453747 \\
\hline 2 & 15.481 & 231610 & 3.48 & 15132 \\
\hline
\end{tabular}


(R,E)-2-(9H-fluoren-2-yl)-2-methylhepta-4,6-dienal (3i)

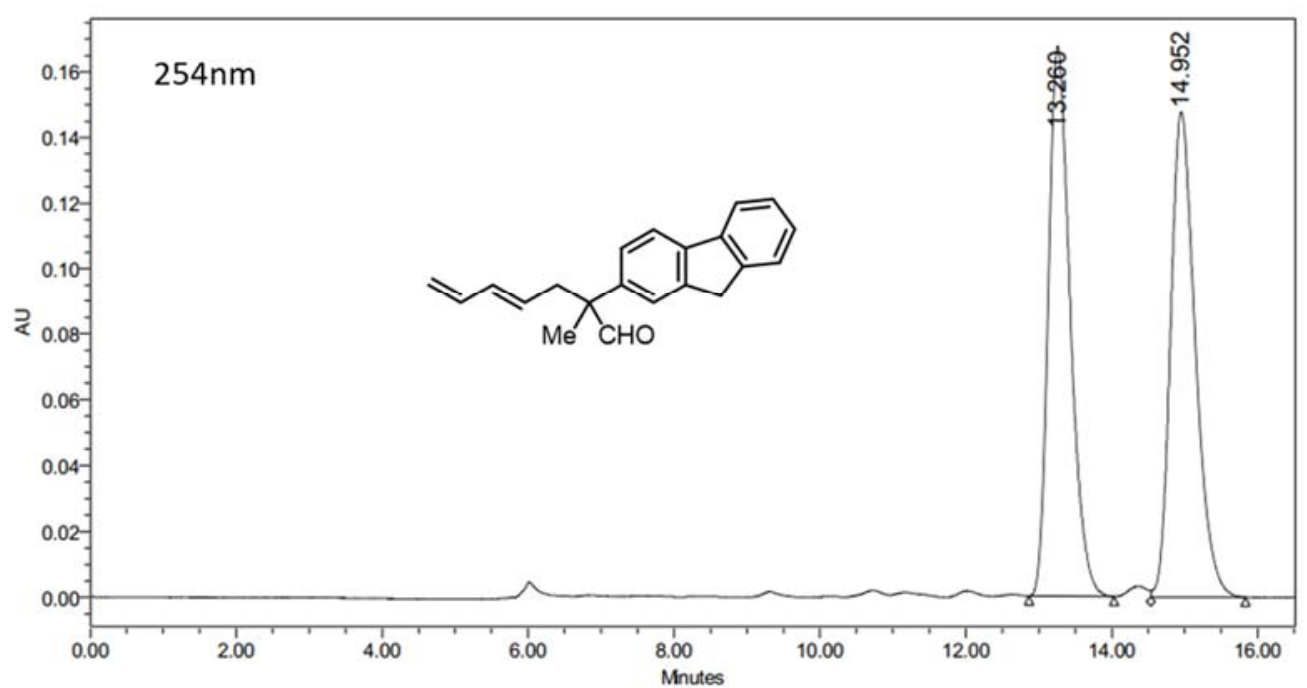

\begin{tabular}{|c|c|c|r|c|}
\hline & RT & Area & $\%$ Area & Height \\
\hline 1 & 13.260 & 3460608 & 50.05 & 167452 \\
\hline 2 & 14.952 & 3453786 & 49.95 & 147751 \\
\hline
\end{tabular}

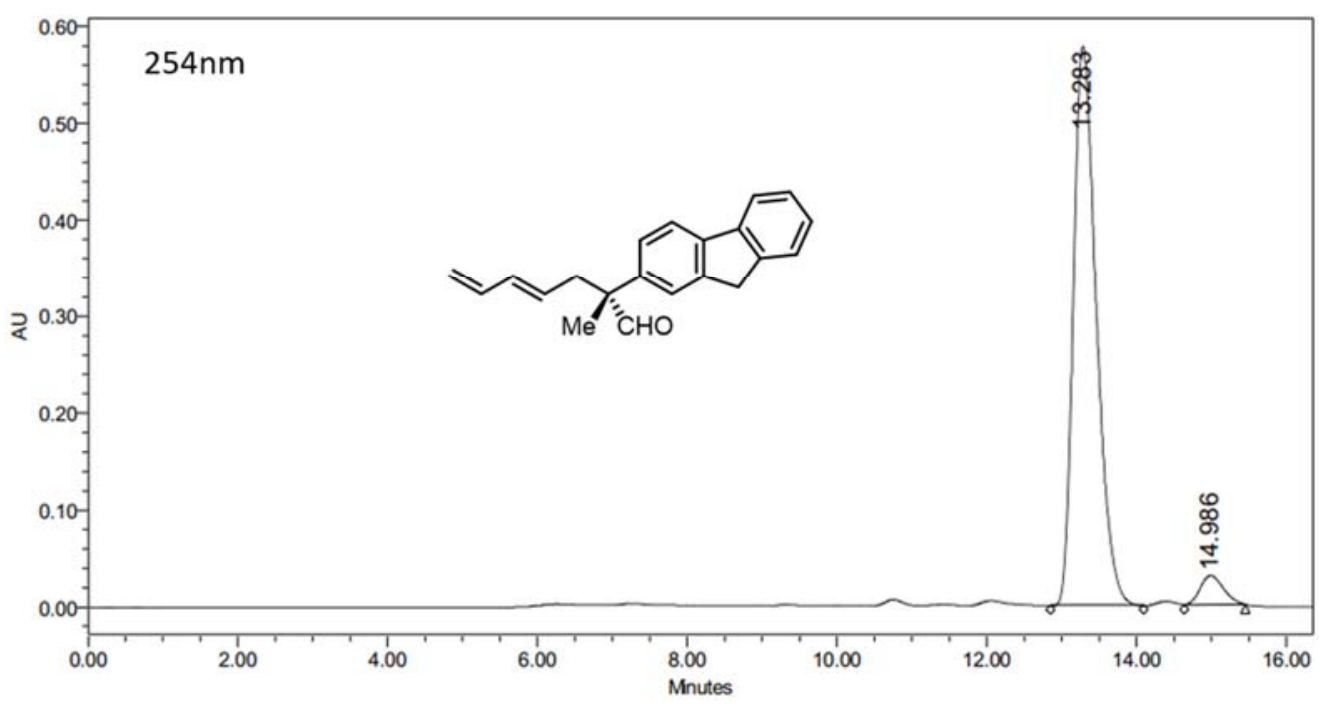

\begin{tabular}{|r|c|r|r|r|}
\hline & RT & \multicolumn{1}{|c|}{ Area } & $\%$ Area & Height \\
\hline 1 & 13.283 & 12130304 & 94.91 & 577142 \\
\hline 2 & 14.986 & 650130 & 5.09 & 30128 \\
\hline
\end{tabular}


(R,E)-2-methyl-2-(naphthalen-2-yl)hepta-4,6-dienal (3j)

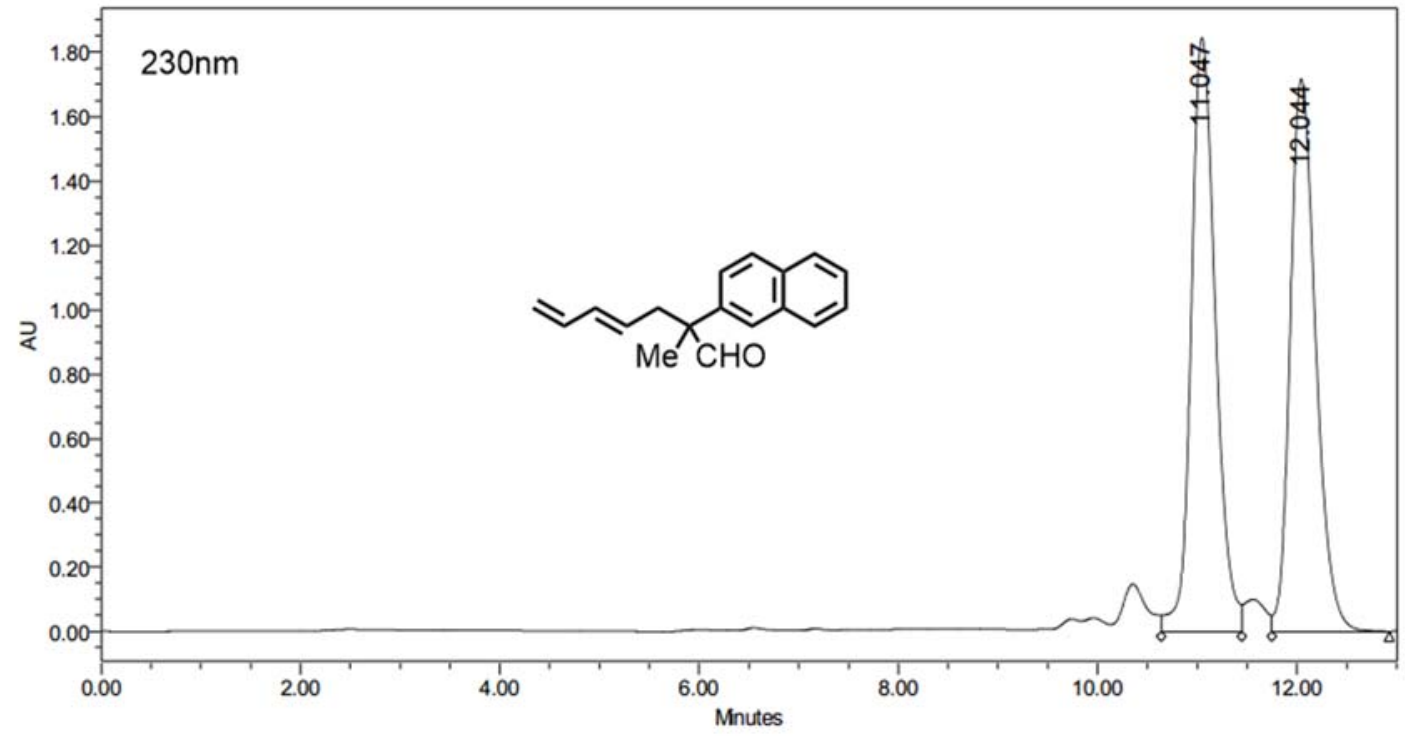

\begin{tabular}{|c|c|c|r|c|}
\hline & RT & Area & $\%$ Area & Height \\
\hline 1 & 11.047 & 31450747 & 50.61 & 1844635 \\
\hline 2 & 12.044 & 30687730 & 49.39 & 1717298 \\
\hline
\end{tabular}

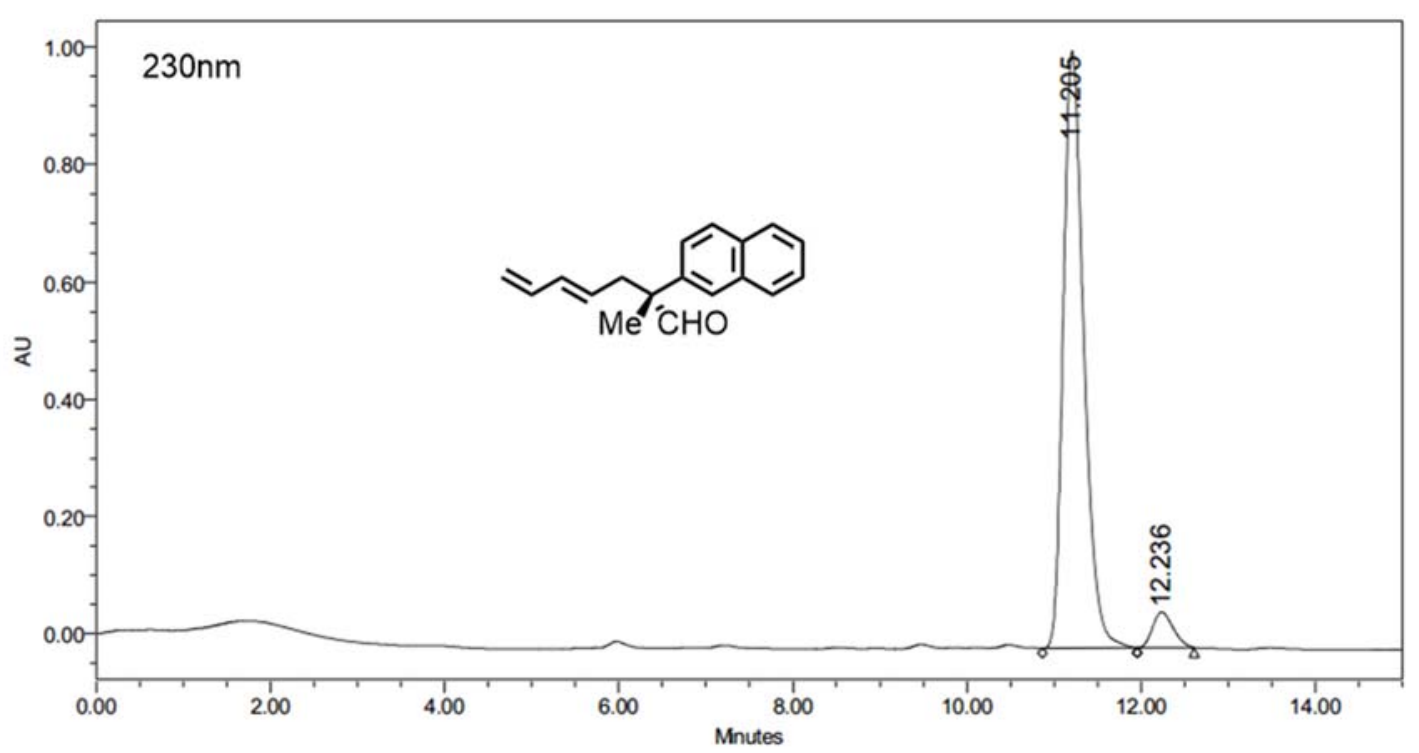

\begin{tabular}{|r|c|r|r|r|}
\hline & RT & \multicolumn{1}{|c|}{ Area } & \% Area & \multicolumn{1}{c|}{ Height } \\
\hline 1 & 11.205 & 16568375 & 94.36 & 1017910 \\
\hline 2 & 12.236 & 989438 & 5.64 & 60388 \\
\hline
\end{tabular}


(R,E)-2-ethyl-2-phenylhepta-4,6-dienal (3k)
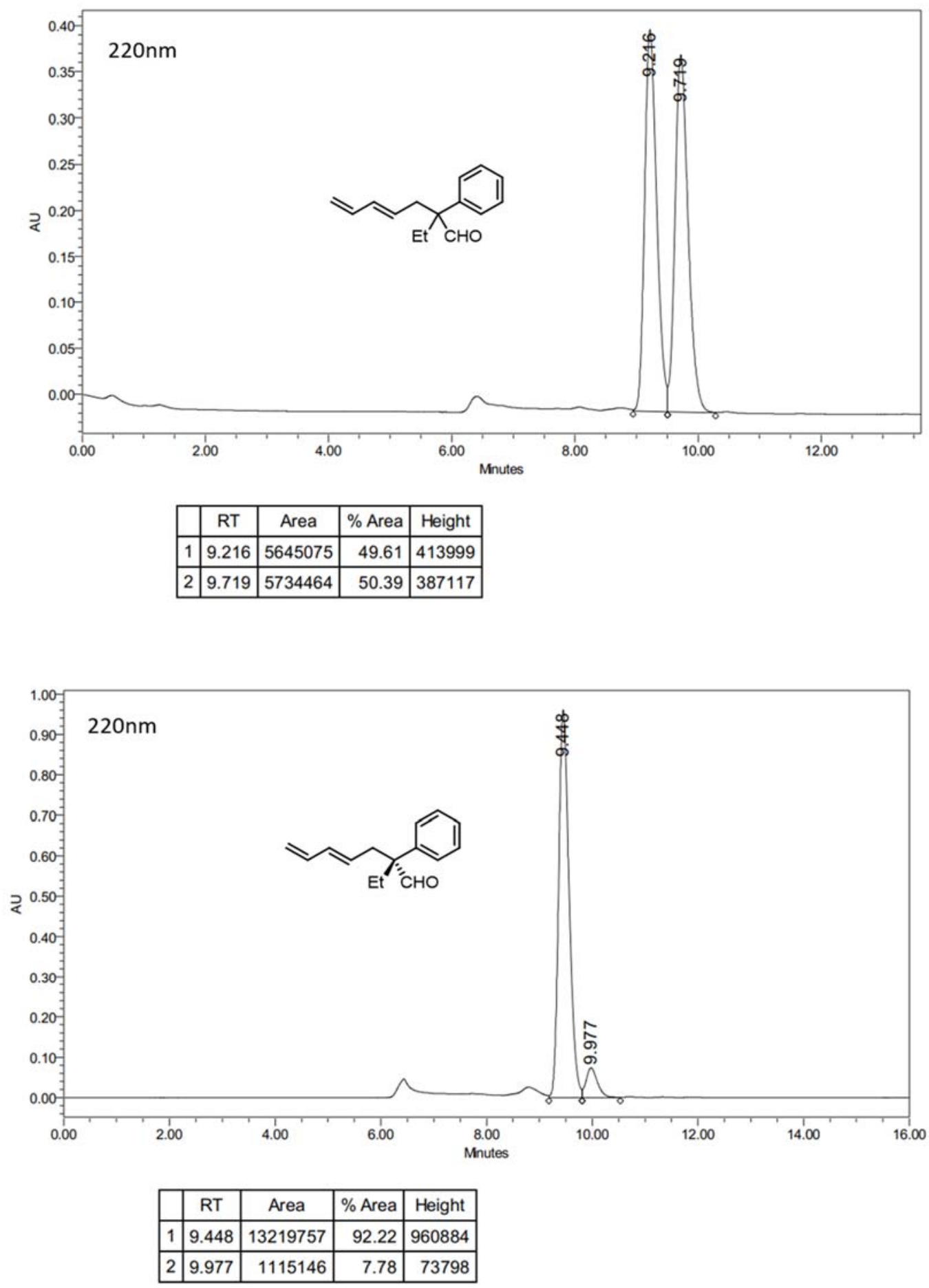
(S,E)-2-cyclopropyl-2-phenylhepta-4,6-dienal (3I)

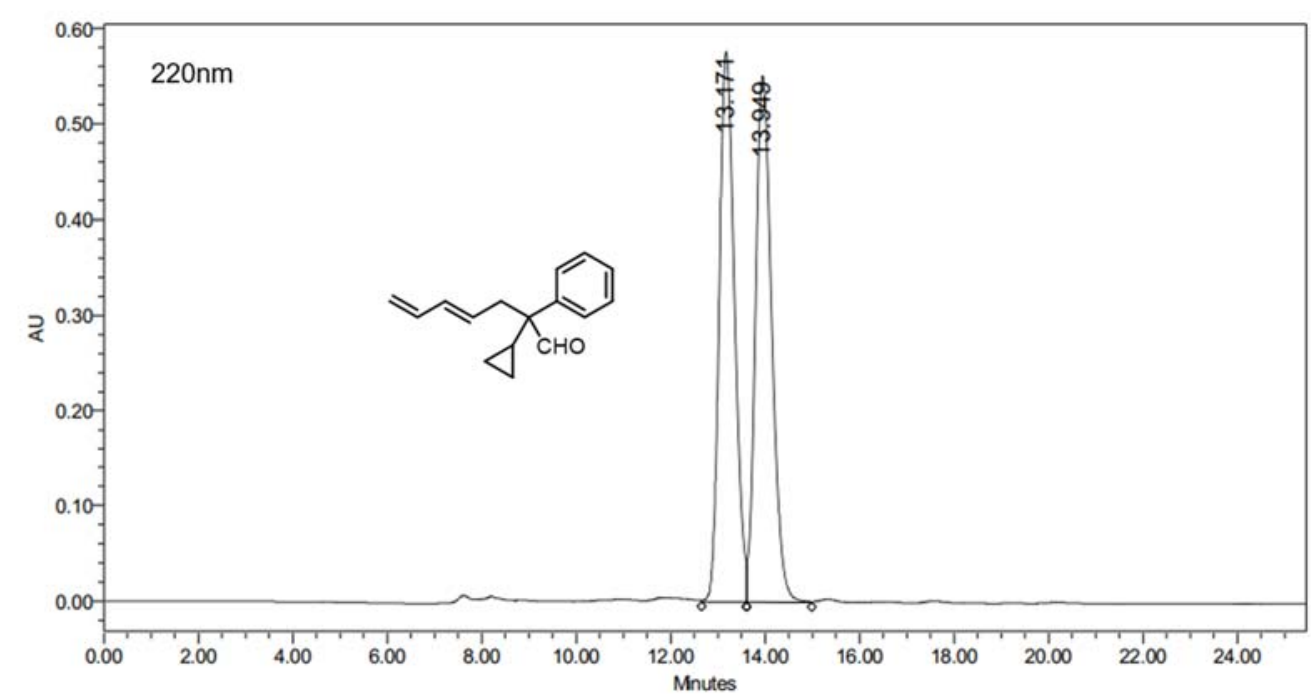

\begin{tabular}{|c|c|c|r|c|}
\hline & RT & Area & $\%$ Area & Height \\
\hline 1 & 13.171 & 12941002 & 49.42 & 576241 \\
\hline 2 & 13.949 & 13244405 & 50.58 & 551215 \\
\hline
\end{tabular}

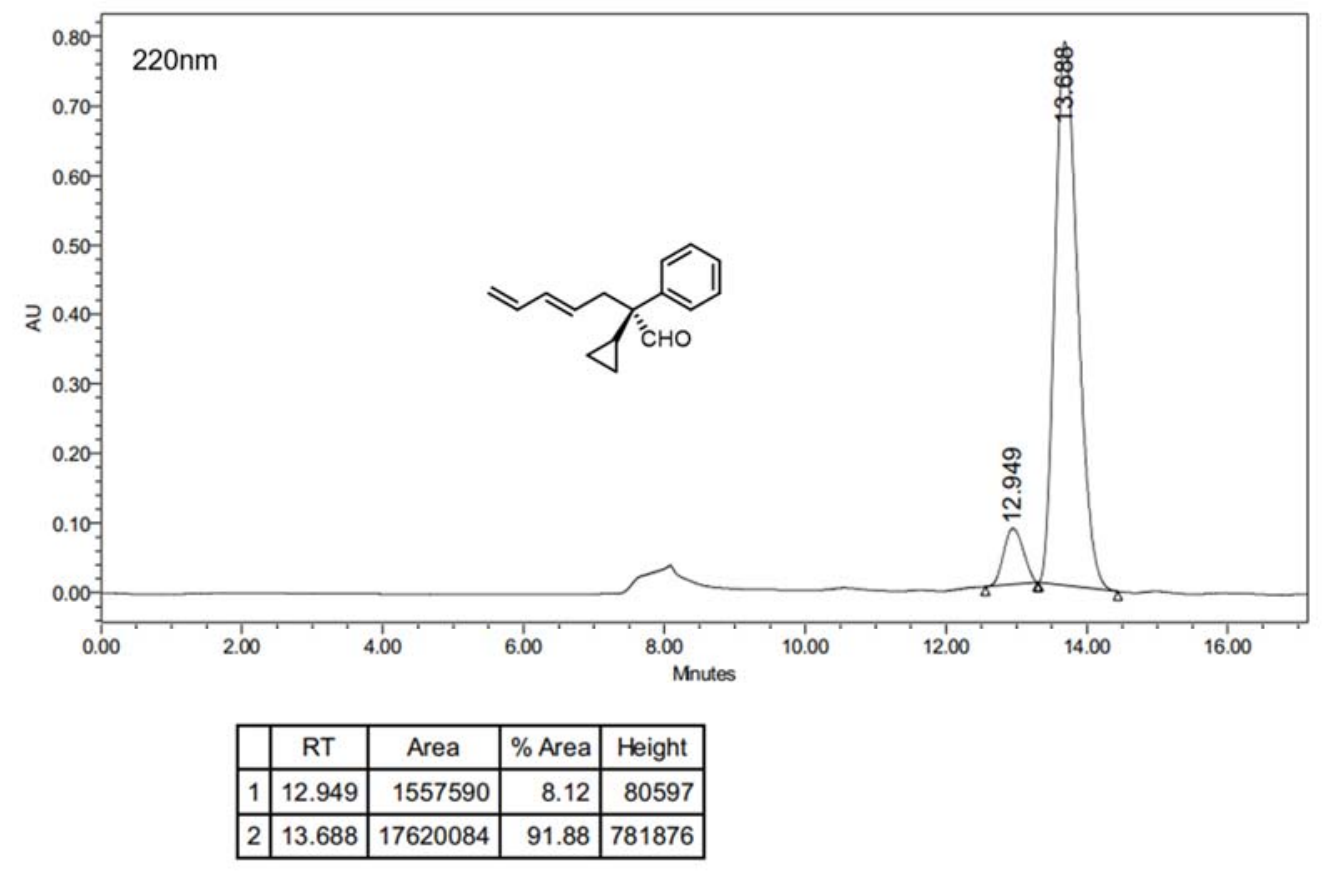


(R,E)-1-(penta-2,4-dien-1-yl)-2,3-dihydro-1H-indene-1-carbaldehyde (3m)

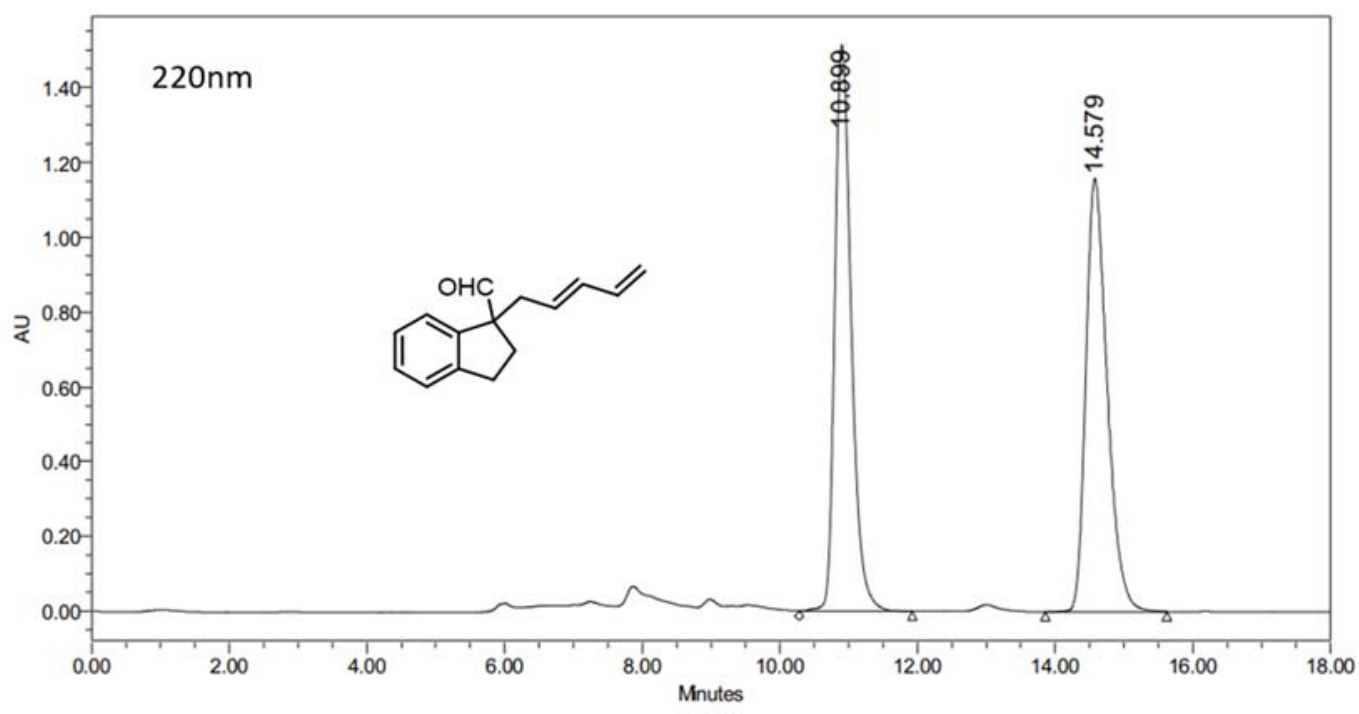

\begin{tabular}{|c|c|c|r|c|}
\hline & RT & Area & $\%$ Area & Height \\
\hline 1 & 10.899 & 25211846 & 50.34 & 1514725 \\
\hline 2 & 14.579 & 24872898 & 49.66 & 1159509 \\
\hline
\end{tabular}

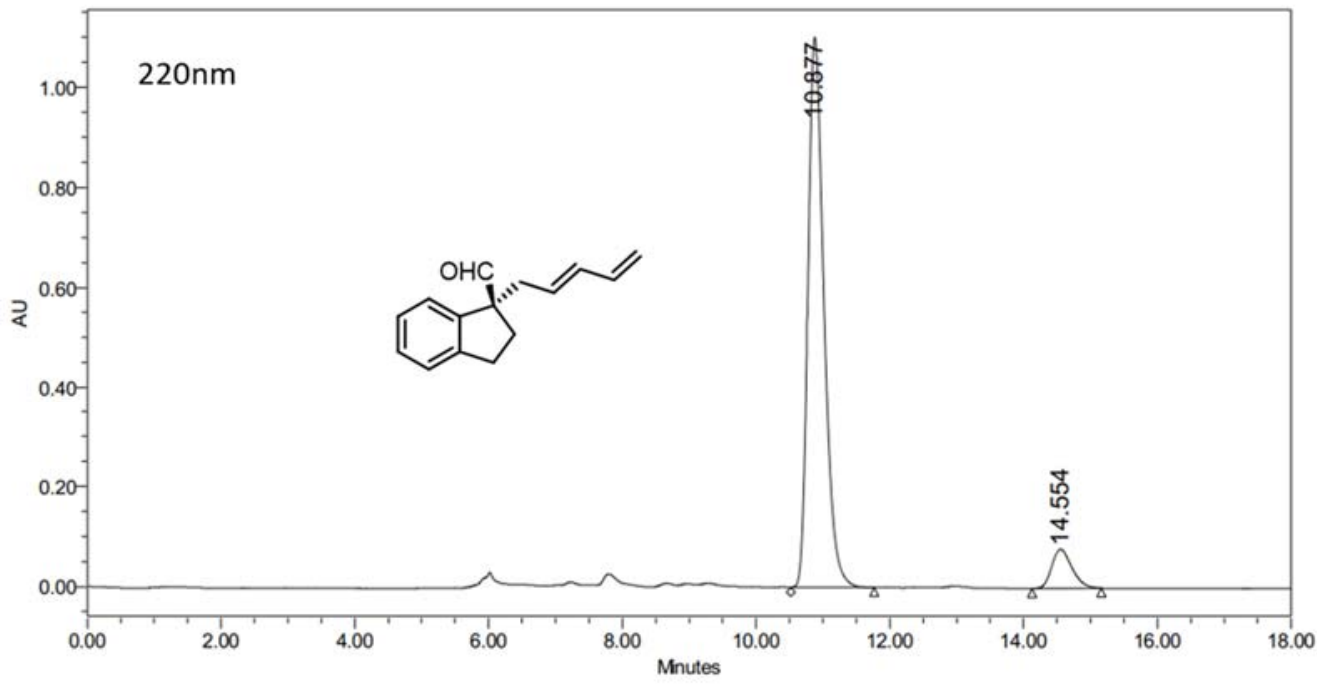

\begin{tabular}{|l|c|r|r|r|}
\hline & RT & Area & \% Area & \multicolumn{1}{c|}{ Height } \\
\hline 1 & 10.877 & 18355893 & 91.85 & 1102149 \\
\hline 2 & 14.554 & 1629366 & 8.15 & 78052 \\
\hline
\end{tabular}


(R,E)-1-(penta-2,4-dien-1-yl)-1,2,3,4-tetrahydronaphthalene-1-carbaldehyde (3n)

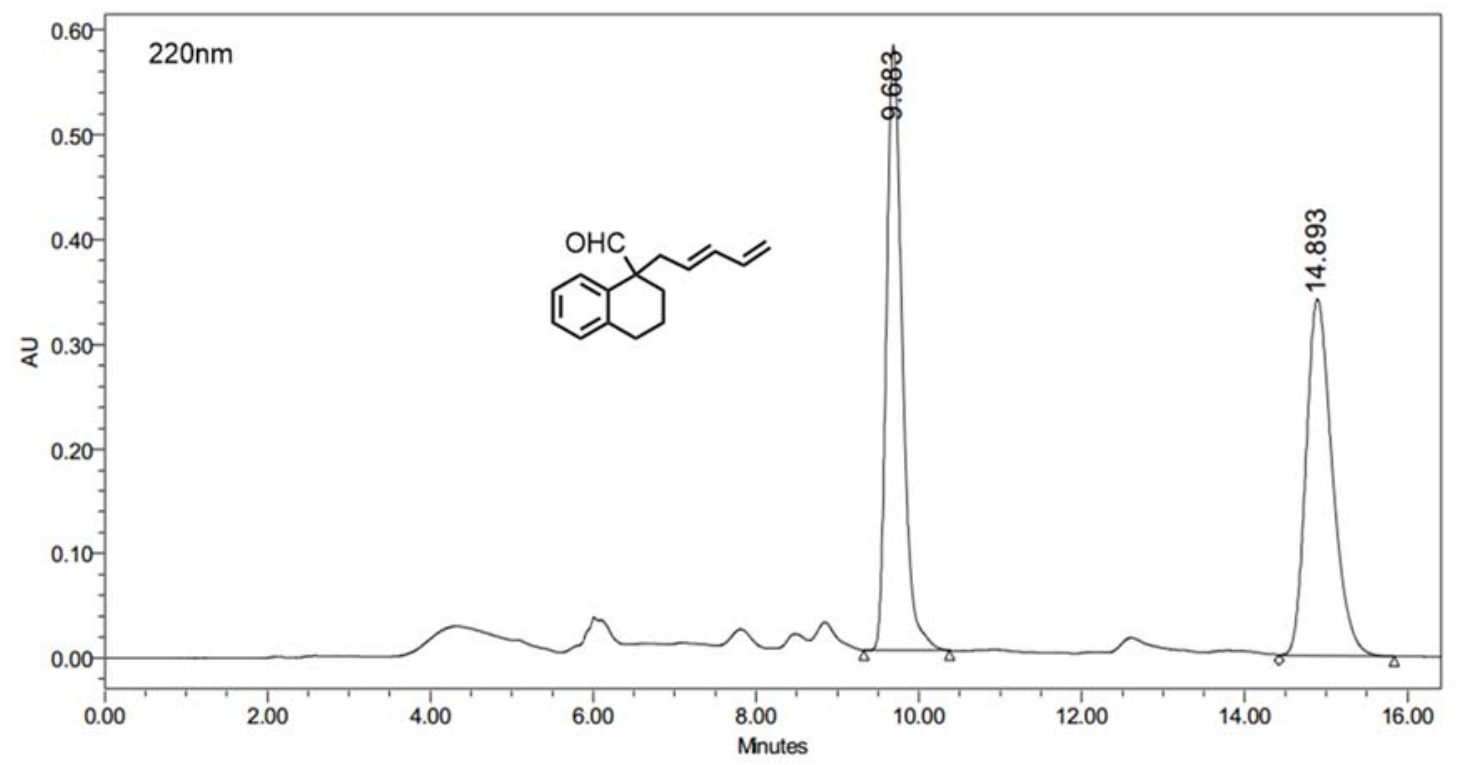

\begin{tabular}{|r|r|c|r|c|}
\hline & \multicolumn{1}{|c|}{ RT } & Area & \% Area & Height \\
\hline 1 & 9.683 & 7781696 & 50.54 & 577990 \\
\hline 2 & 14.893 & 7614800 & 49.46 & 341162 \\
\hline
\end{tabular}

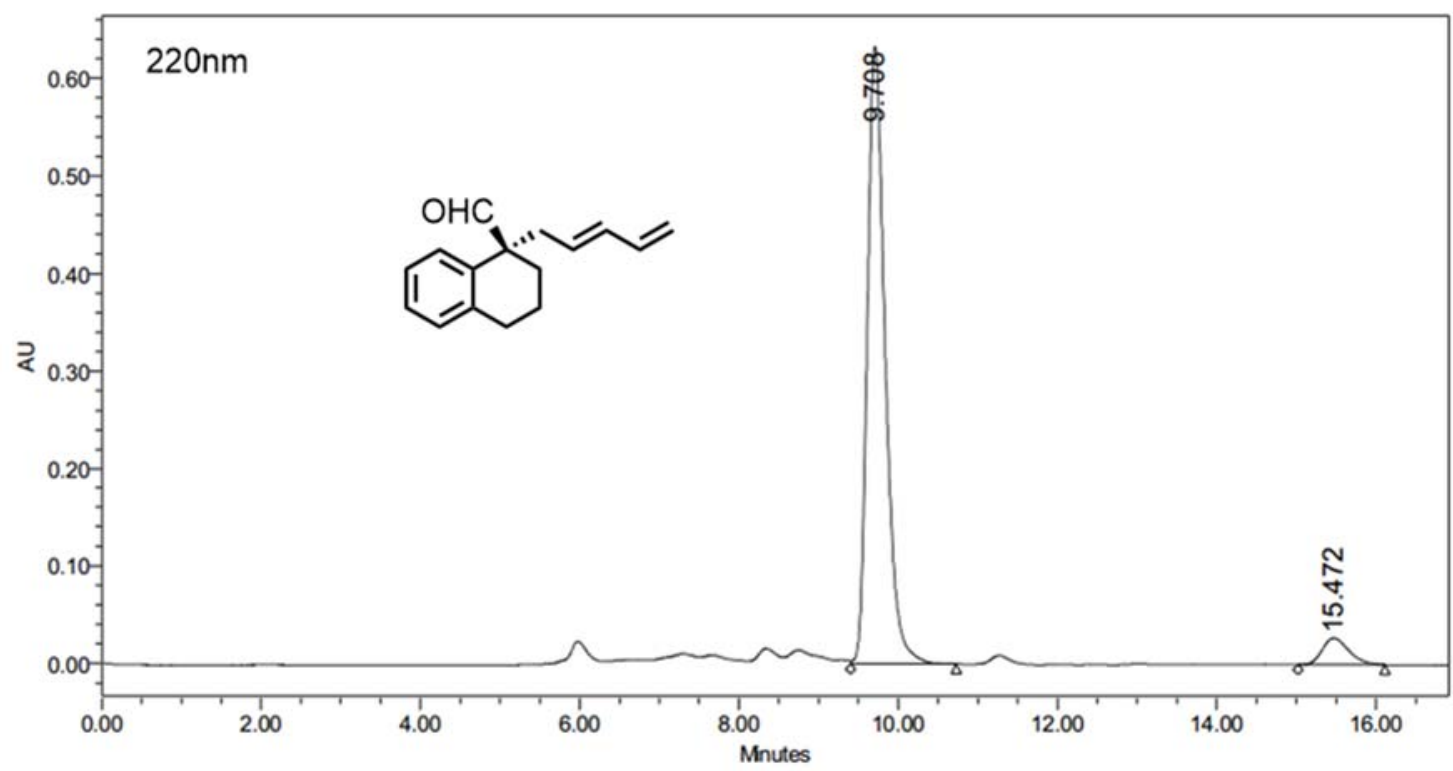

\begin{tabular}{|r|r|r|r|r|}
\hline & \multicolumn{1}{|c|}{ RT } & \multicolumn{1}{c|}{ Area } & \% Area & Height \\
\hline 1 & 9.708 & 9796538 & 93.94 & 632852 \\
\hline 2 & 15.472 & 632186 & 6.06 & 27380 \\
\hline
\end{tabular}



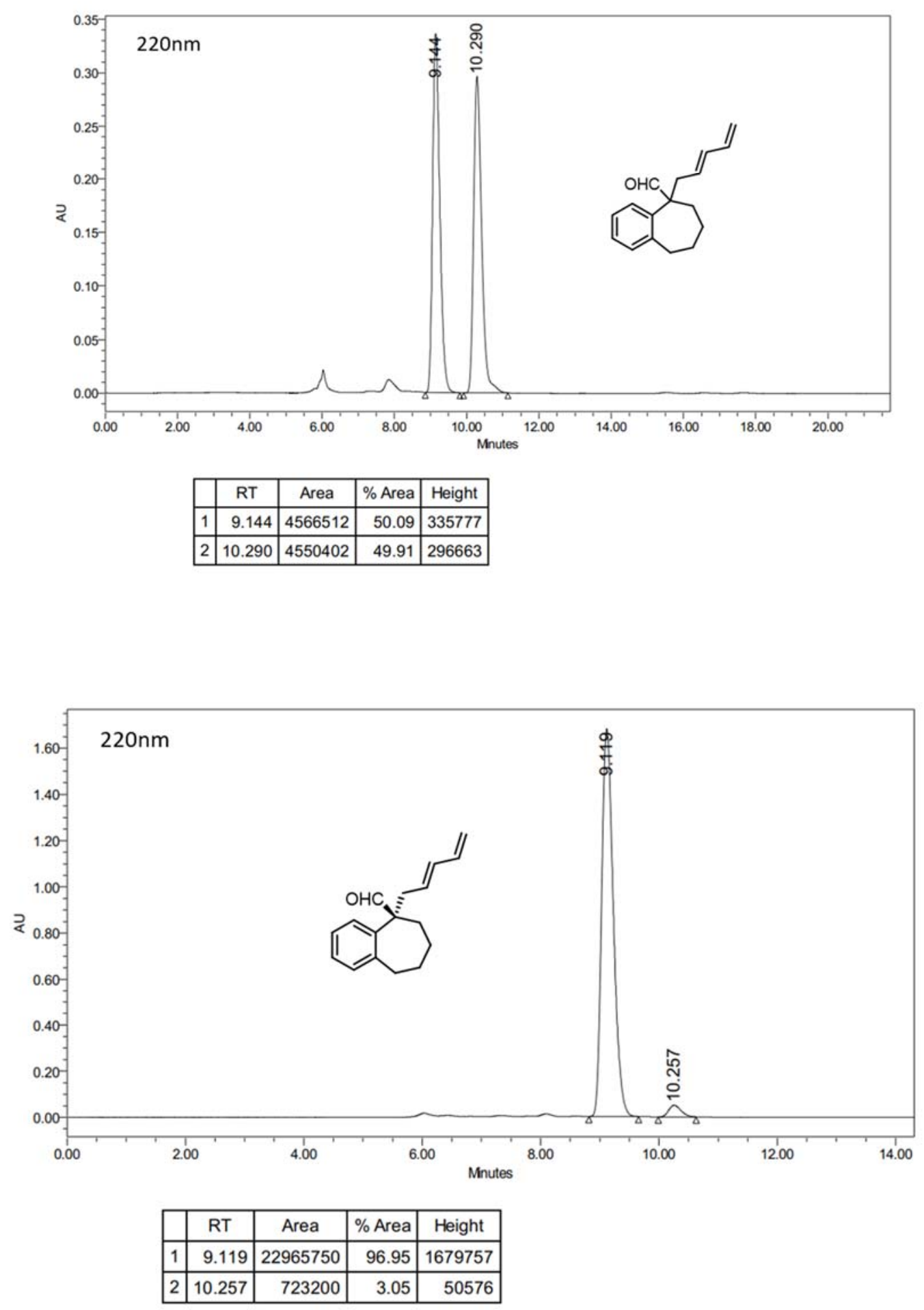
(R,E)-2-methyl-2-(pyridin-2-yl)hepta-4,6-dienal (3p)

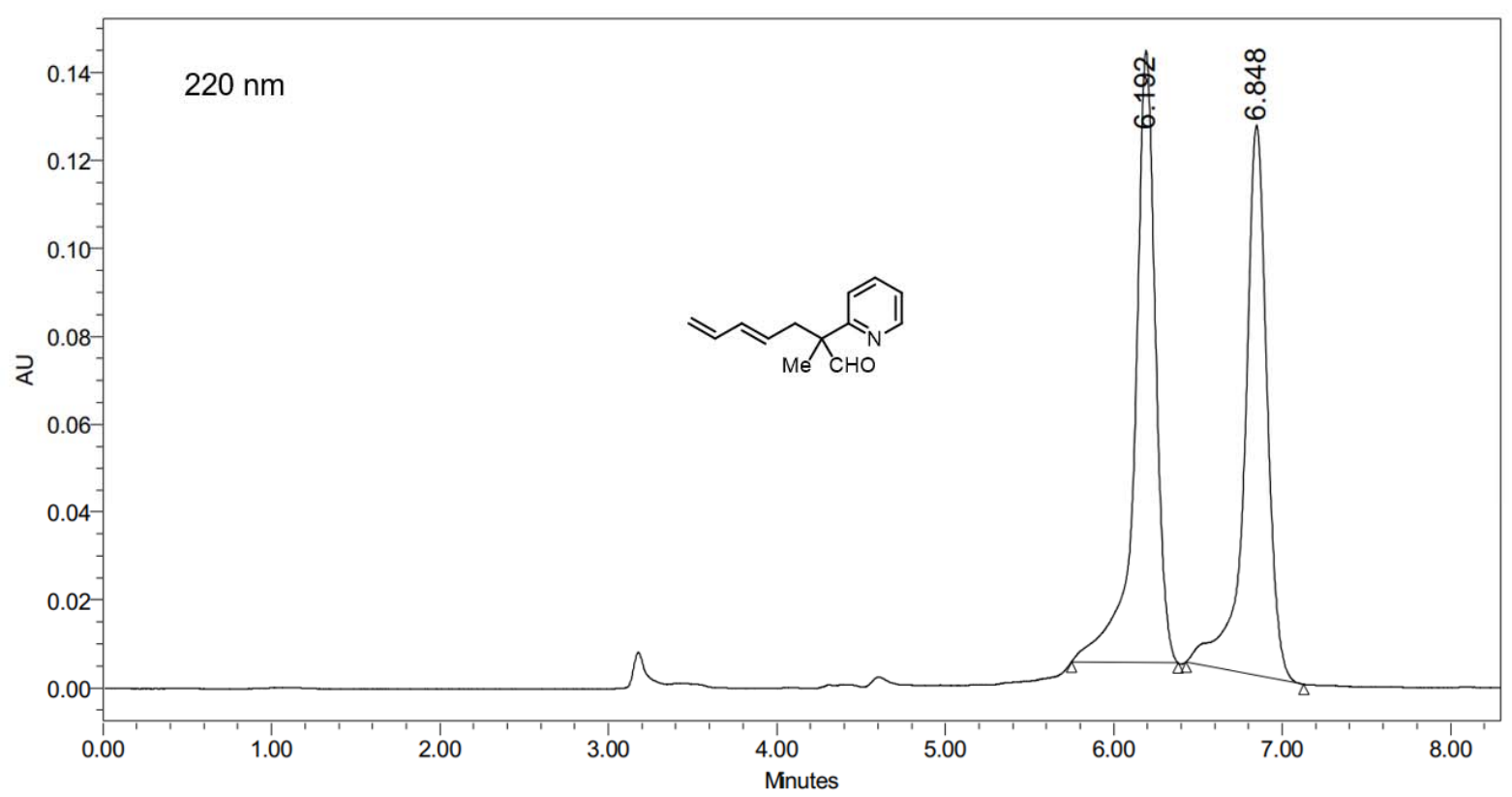

\begin{tabular}{|c|c|c|r|c|}
\hline & RT & Area & $\%$ Area & Height \\
\hline 1 & 6.192 & 1182181 & 50.53 & 139151 \\
\hline 2 & 6.848 & 1157321 & 49.47 & 125050 \\
\hline
\end{tabular}

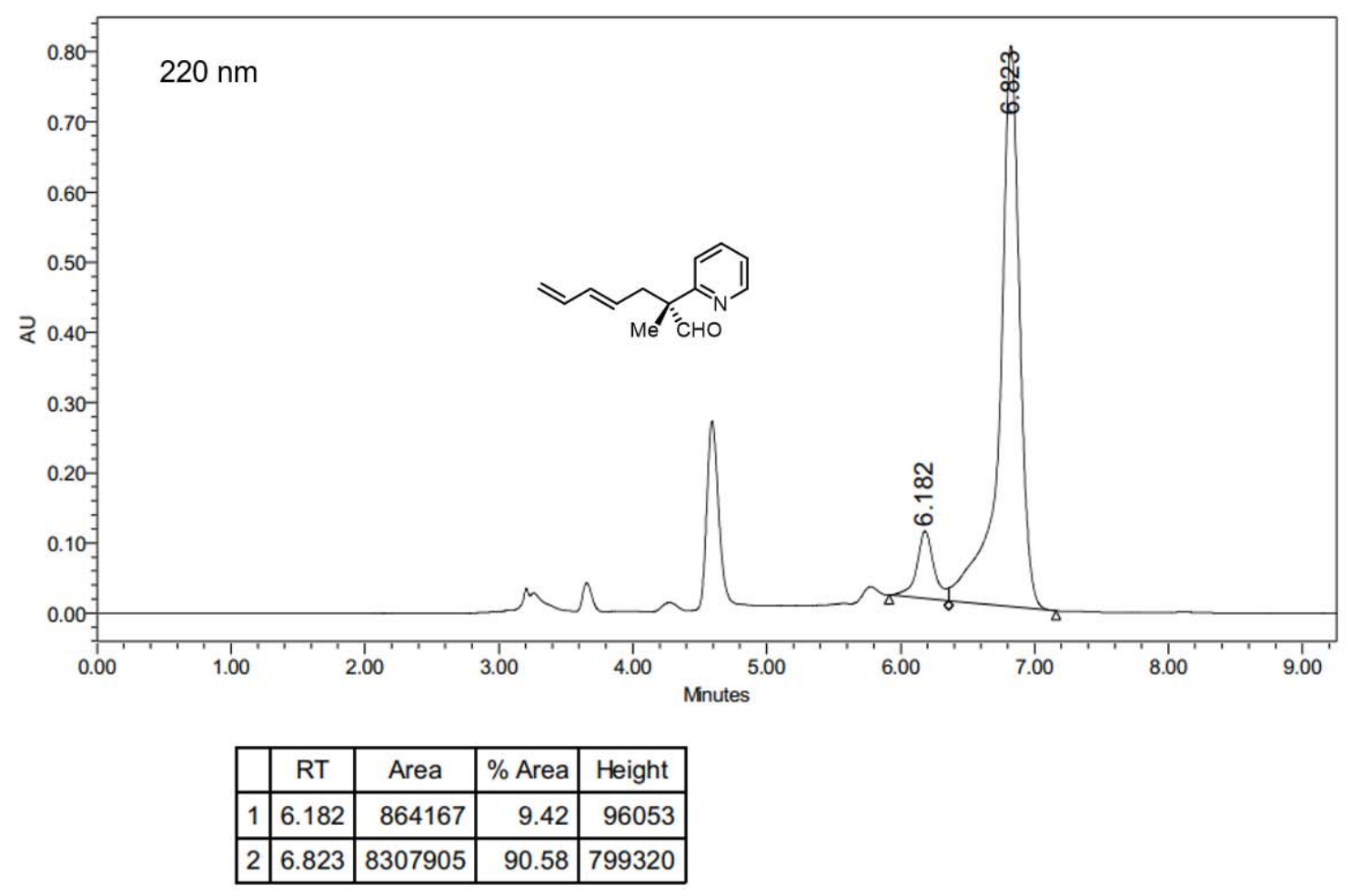


(S,E)-2-methyl-2-(thiophen-2-yl)hepta-4,6-dienal (3q)

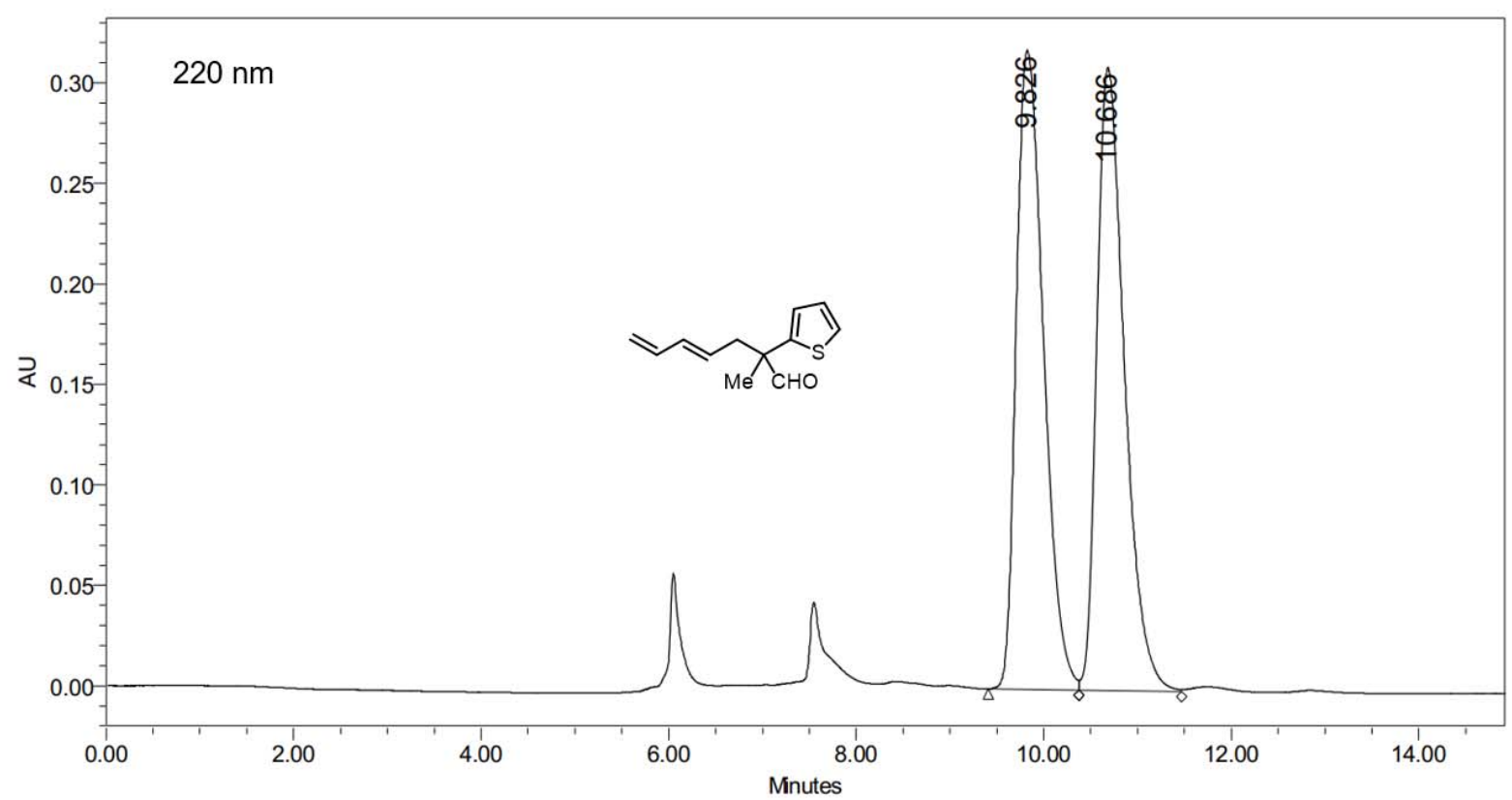

\begin{tabular}{|c|c|c|r|c|}
\hline & \multicolumn{1}{|c|}{ RT } & Area & $\%$ Area & Height \\
\hline 1 & 9.826 & 6390409 & 50.18 & 318027 \\
\hline 2 & 10.686 & 6344220 & 49.82 & 309979 \\
\hline
\end{tabular}

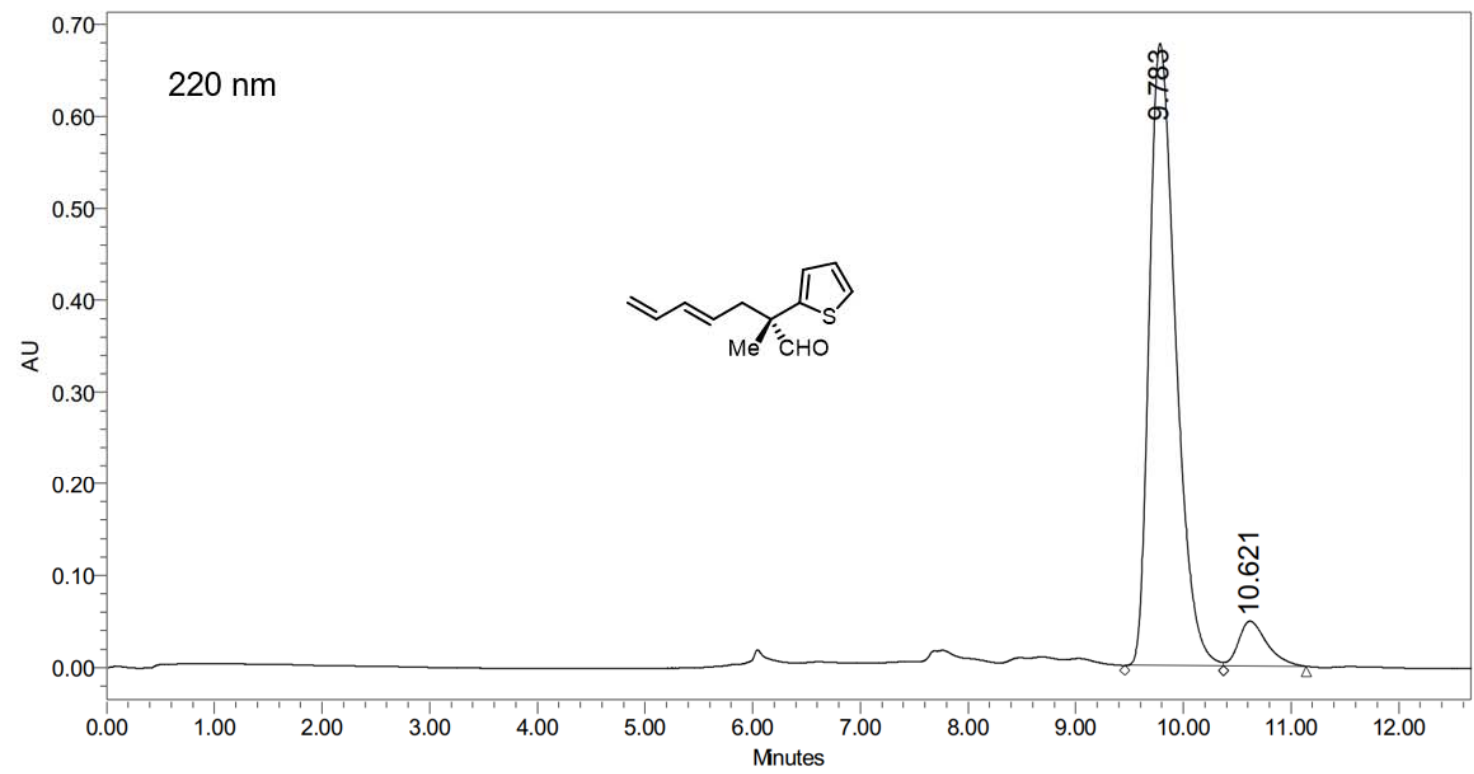

\begin{tabular}{|l|r|r|r|r|}
\hline & \multicolumn{1}{|c|}{ RT } & \multicolumn{1}{c|}{ Area } & \% Area & Height \\
\hline 1 & 9.783 & 11616315 & 92.88 & 676650 \\
\hline 2 & 10.621 & 890958 & 7.12 & 48672 \\
\hline
\end{tabular}


(R,4E,6E)-2-methyl-2,7-diphenylhepta-4,6-dienal (3r)
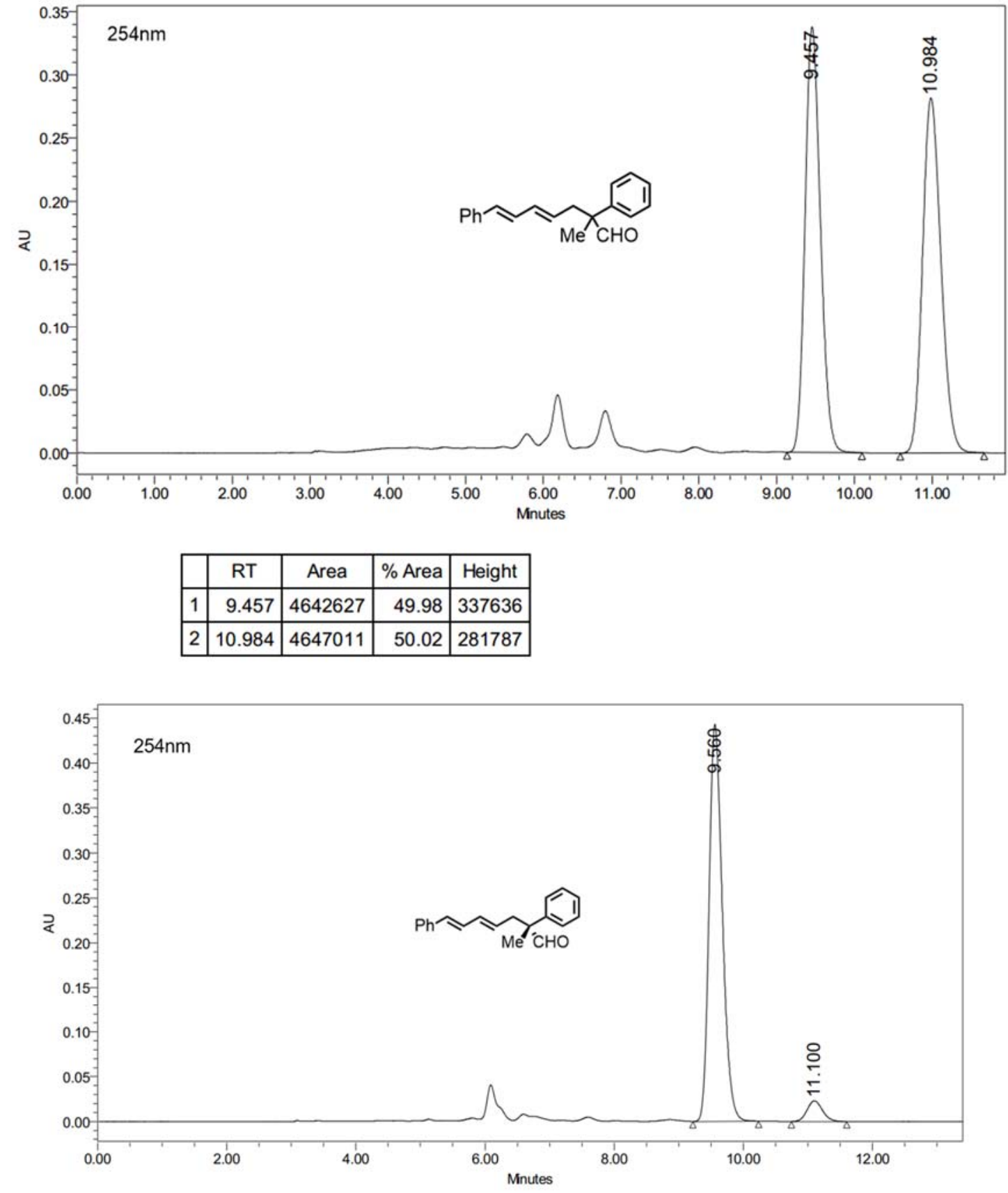

\begin{tabular}{|r|r|r|r|r|}
\hline & \multicolumn{1}{|c|}{ RT } & \multicolumn{1}{c|}{ Area } & $\%$ Area & Height \\
\hline 1 & 9.560 & 6306586 & 94.30 & 442853 \\
\hline 2 & 11.100 & 381525 & 5.70 & 22995 \\
\hline
\end{tabular}


(R,4E,6E)-2-methyl-2,7-diphenylhepta-4,6-dienal (3r)-Method D
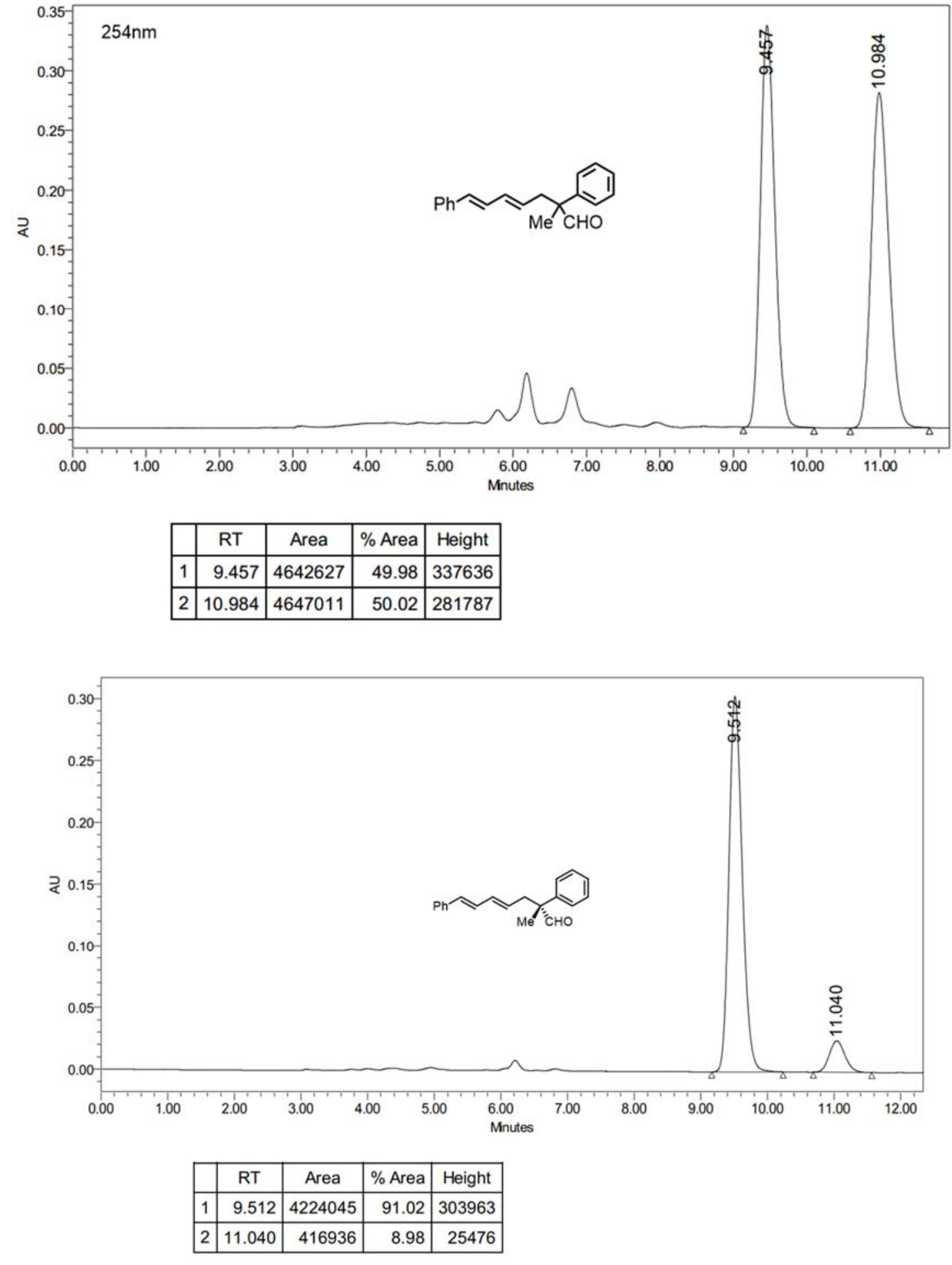
(R,4E,6E)-2-methyl-2-phenyl-7-(p-tolyl)hepta-4,6-dienal (3s)

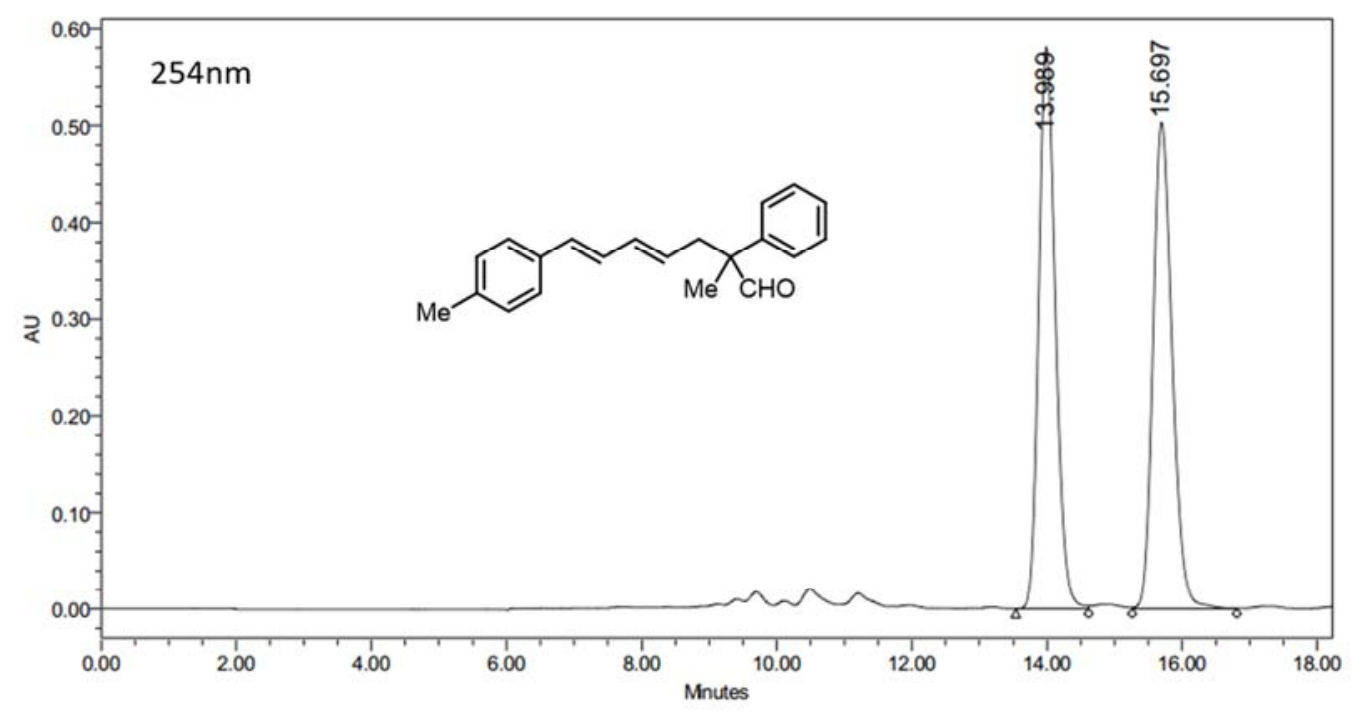

\begin{tabular}{|c|c|c|r|c|}
\hline & RT & Area & $\%$ Area & Height \\
\hline 1 & 13.989 & 10041403 & 50.11 & 580815 \\
\hline 2 & 15.697 & 9996814 & 49.89 & 503559 \\
\hline
\end{tabular}

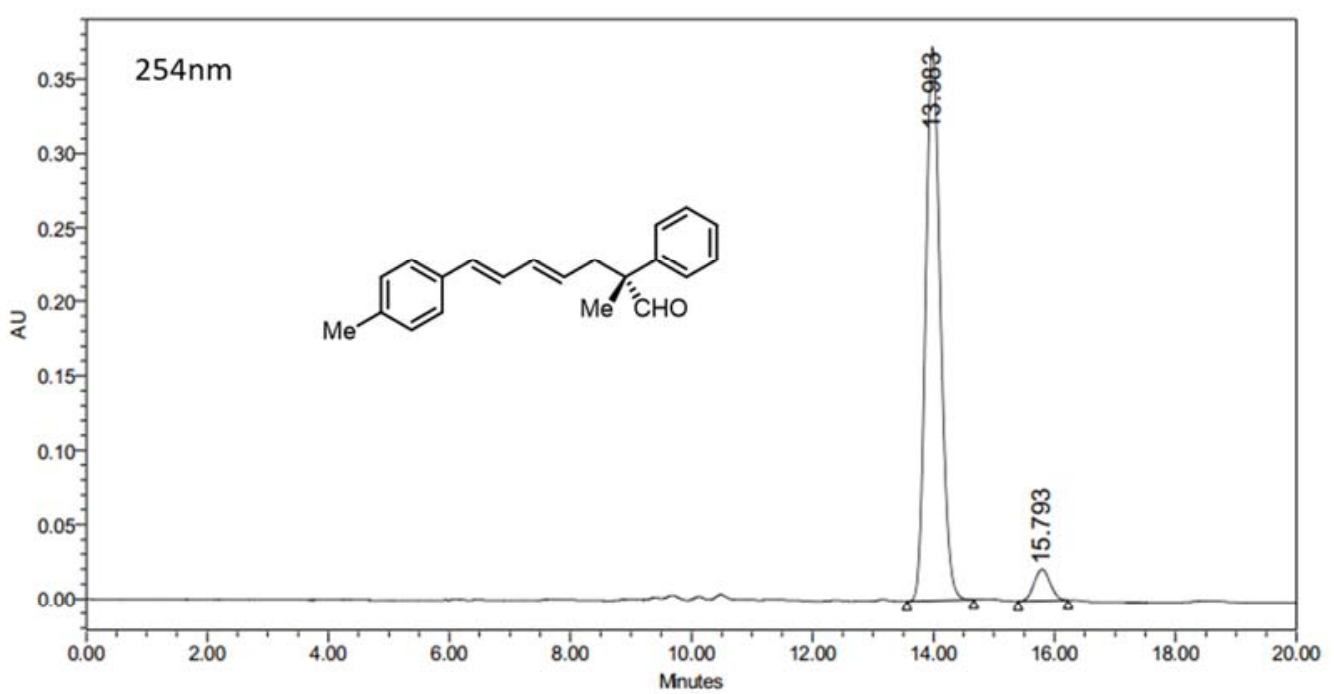

\begin{tabular}{|c|c|c|r|r|}
\hline & RT & Area & $\%$ Area & Height \\
\hline 1 & 13.983 & 6348623 & 94.14 & 372744 \\
\hline 2 & 15.793 & 394894 & 5.86 & 21334 \\
\hline
\end{tabular}


(R,4E,6E)-7-(4-fluorophenyl)-2-methyl-2-phenylhepta-4,6-dienal (3t)

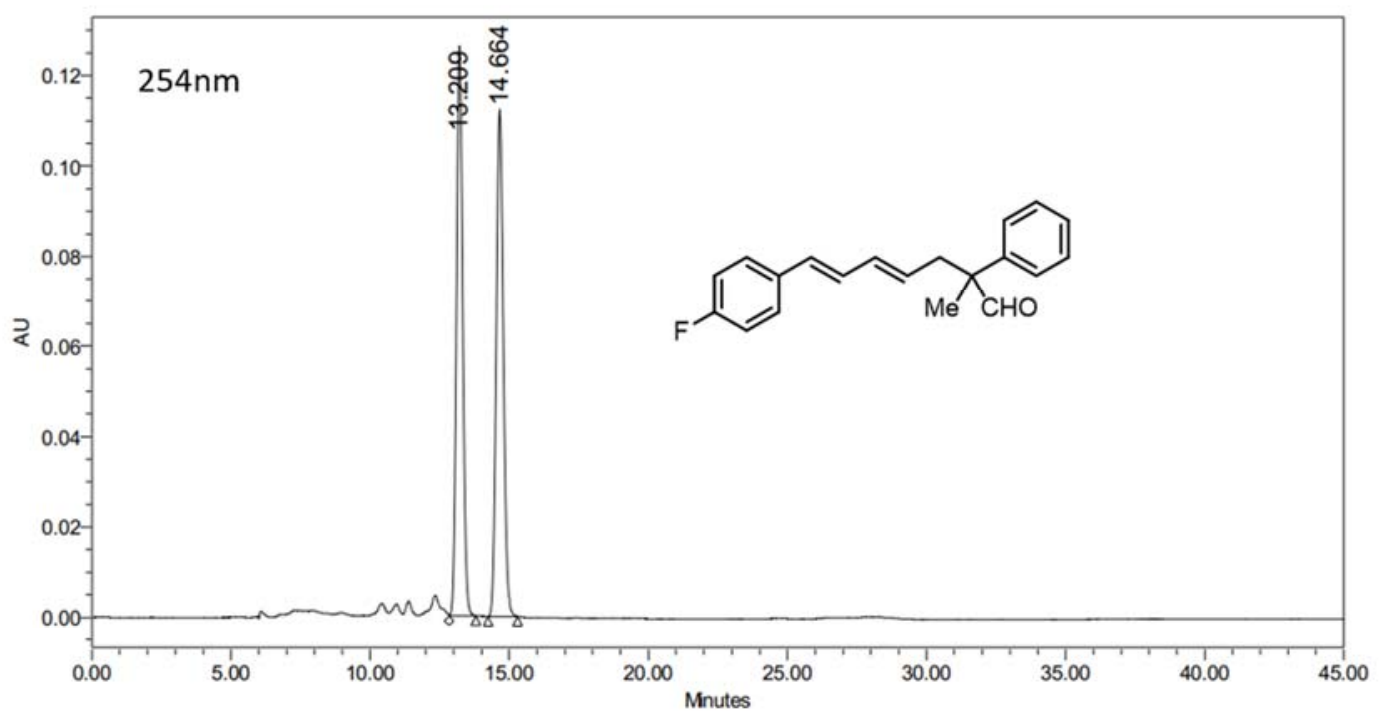

\begin{tabular}{|c|c|c|r|c|}
\hline & RT & Area & $\%$ Area & Height \\
\hline 1 & 13.209 & 1956170 & 49.96 & 126195 \\
\hline 2 & 14.664 & 1959369 & 50.04 & 112255 \\
\hline
\end{tabular}

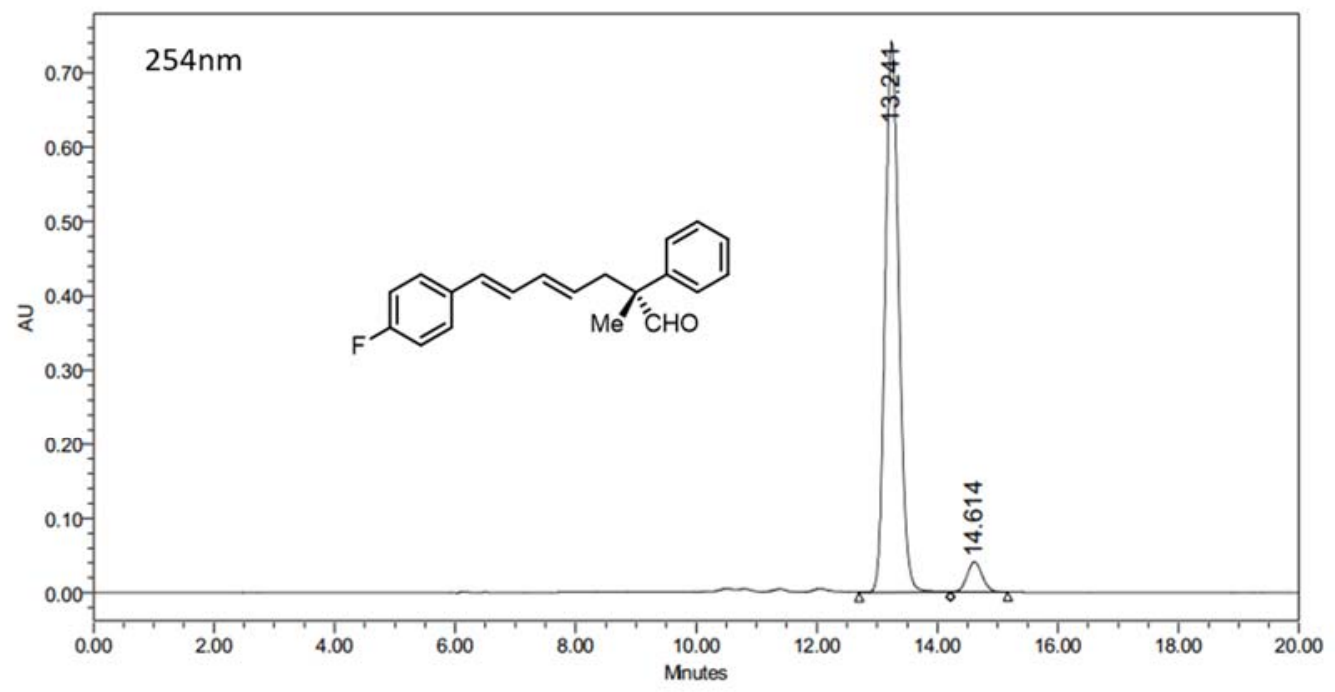

\begin{tabular}{|r|c|r|r|r|}
\hline & RT & \multicolumn{1}{c|}{ Area } & $\%$ Area & Height \\
\hline 1 & 13.241 & 11667487 & 94.20 & 741379 \\
\hline 2 & 14.614 & 717929 & 5.80 & 40702 \\
\hline
\end{tabular}




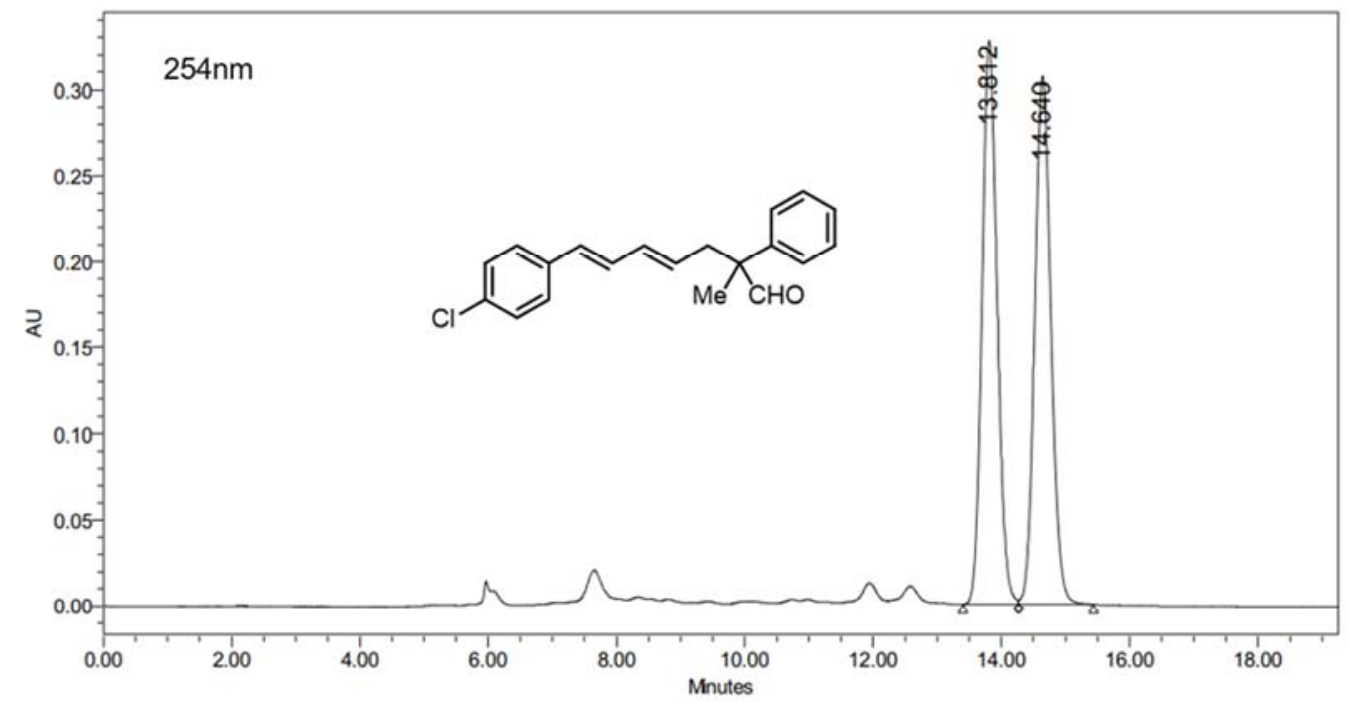

\begin{tabular}{|c|c|c|r|c|}
\hline & RT & Area & \% Area & Height \\
\hline 1 & 13.812 & 5353319 & 49.82 & 327264 \\
\hline 2 & 14.640 & 5391962 & 50.18 & 306735 \\
\hline
\end{tabular}

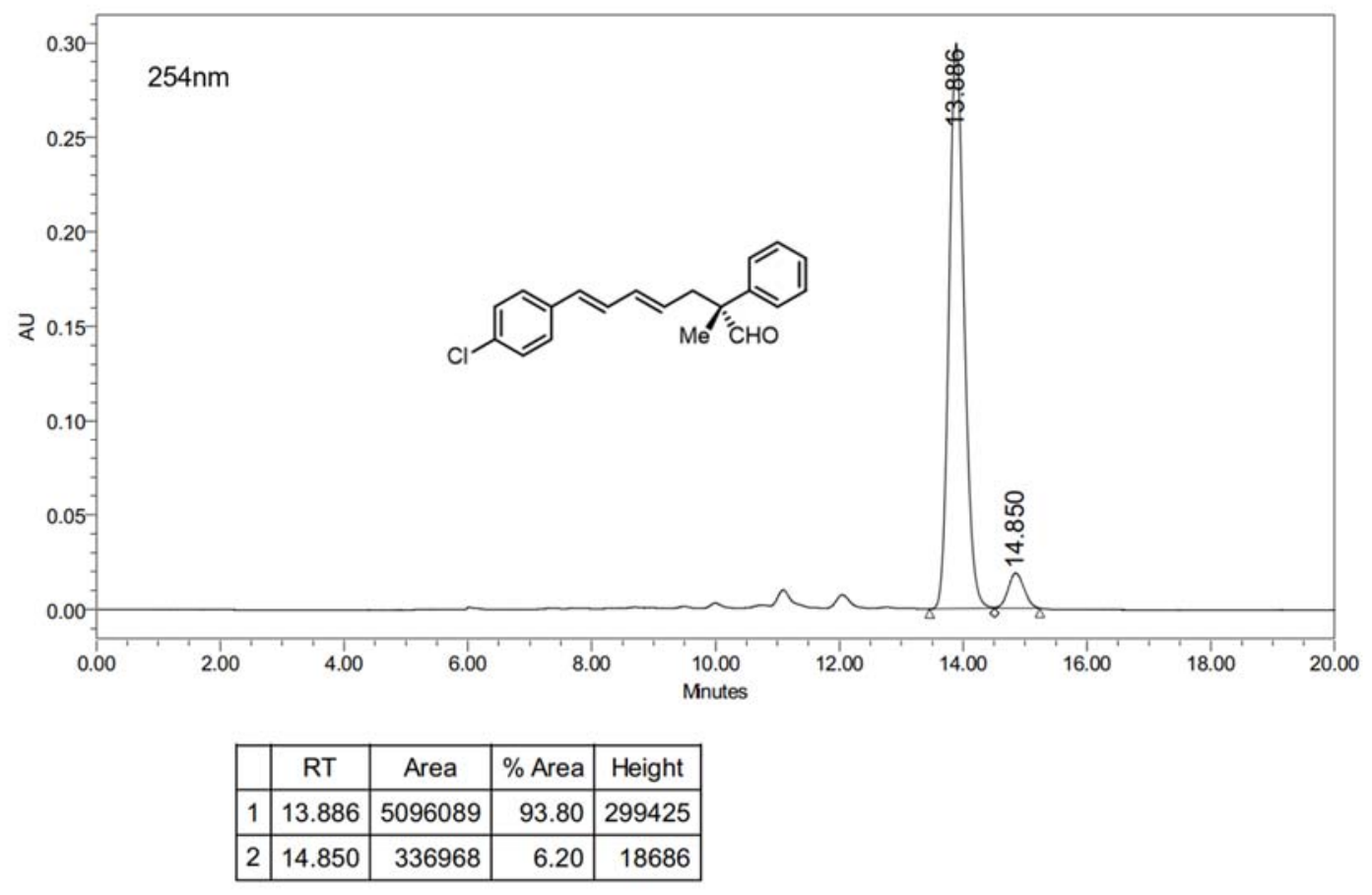


(R,4E,6E)-2-methyl-2-phenyl-7-(4-(trifluoromethyl)phenyl)hepta-4,6-dienal (3v)

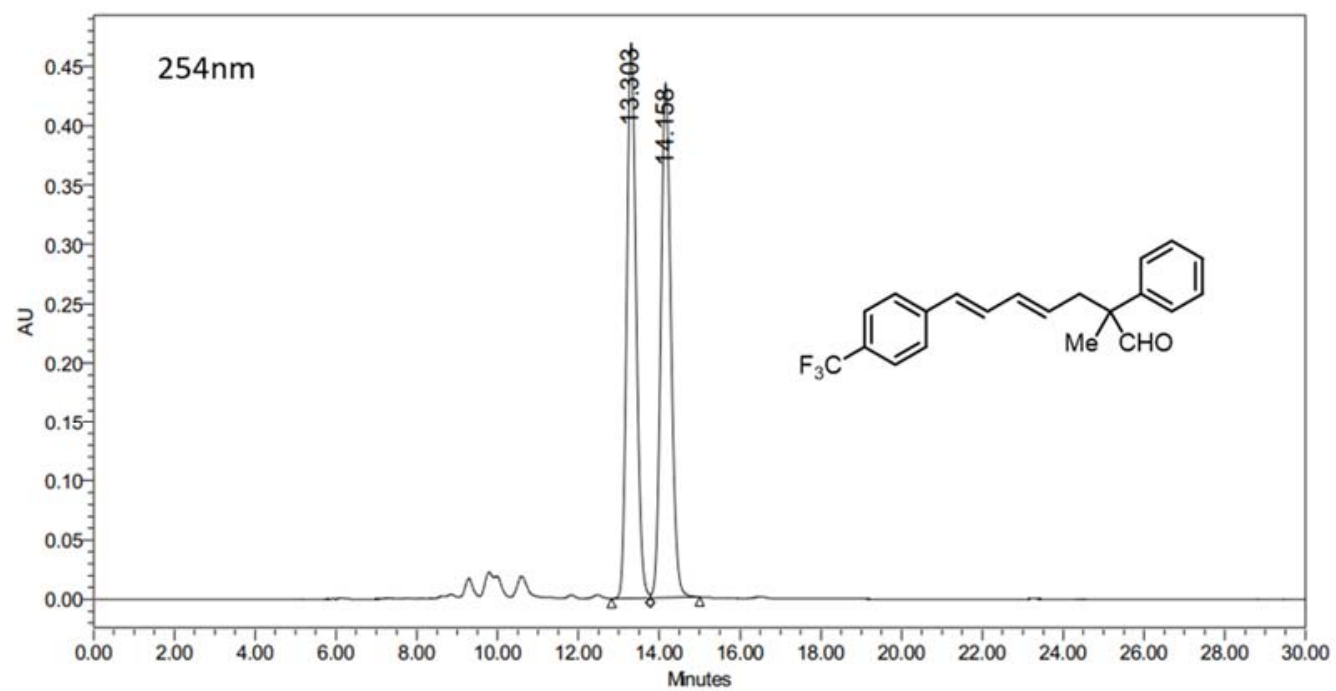

\begin{tabular}{|c|c|c|r|c|}
\hline & RT & Area & $\%$ Area & Height \\
\hline 1 & 13.303 & 7579063 & 49.74 & 468753 \\
\hline 2 & 14.158 & 7656993 & 50.26 & 434853 \\
\hline
\end{tabular}

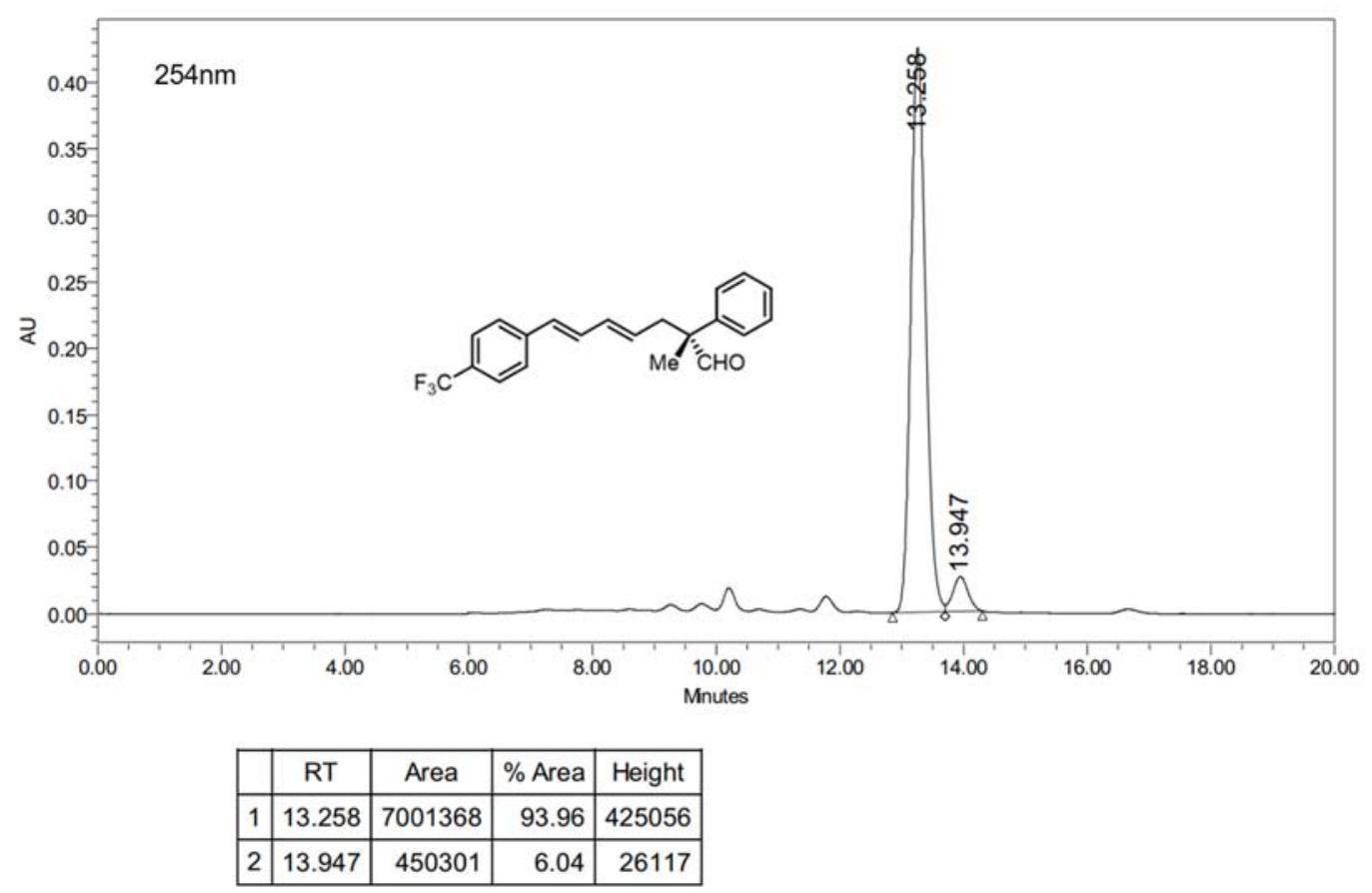


(R,4E,6E)-7-(3-fluorophenyl)-2-methyl-2-phenylhepta-4,6-dienal (3w)

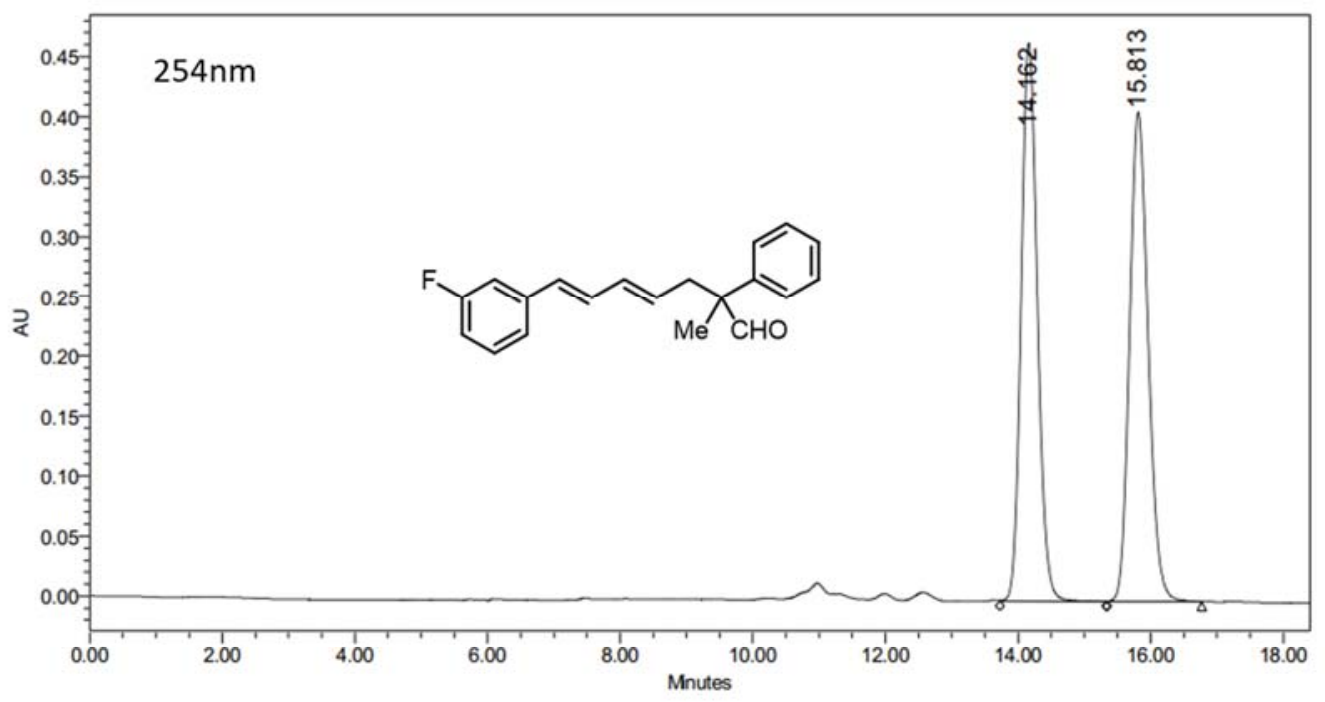

\begin{tabular}{|c|c|c|r|c|}
\hline & RT & Area & $\%$ Area & Height \\
\hline 1 & 14.162 & 7968022 & 49.97 & 465910 \\
\hline 2 & 15.813 & 7978452 & 50.03 & 408266 \\
\hline
\end{tabular}

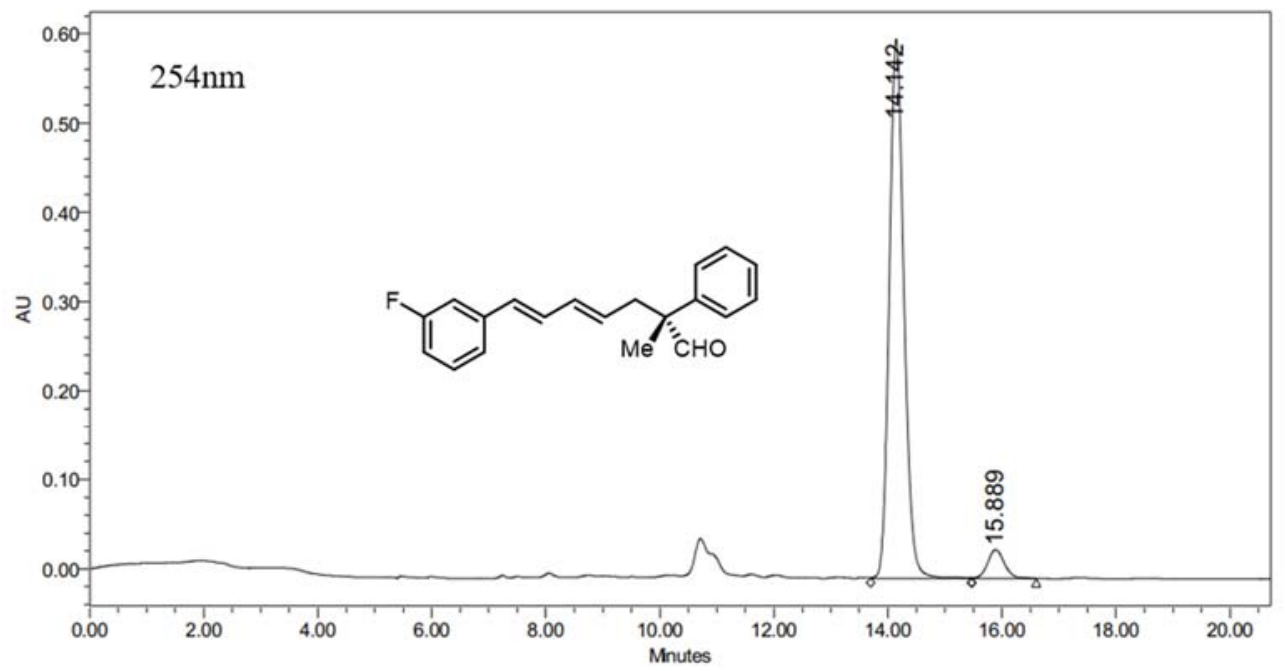

\begin{tabular}{|r|c|r|r|r|}
\hline & RT & \multicolumn{1}{|c|}{ Area } & $\%$ Area & Height \\
\hline 1 & 14.142 & 10813558 & 94.31 & 604476 \\
\hline 2 & 15.889 & 652585 & 5.69 & 32044 \\
\hline
\end{tabular}


(R,4E,6E)-7-(3-chlorophenyl)-2-methyl-2-phenylhepta-4,6-dienal (3x)

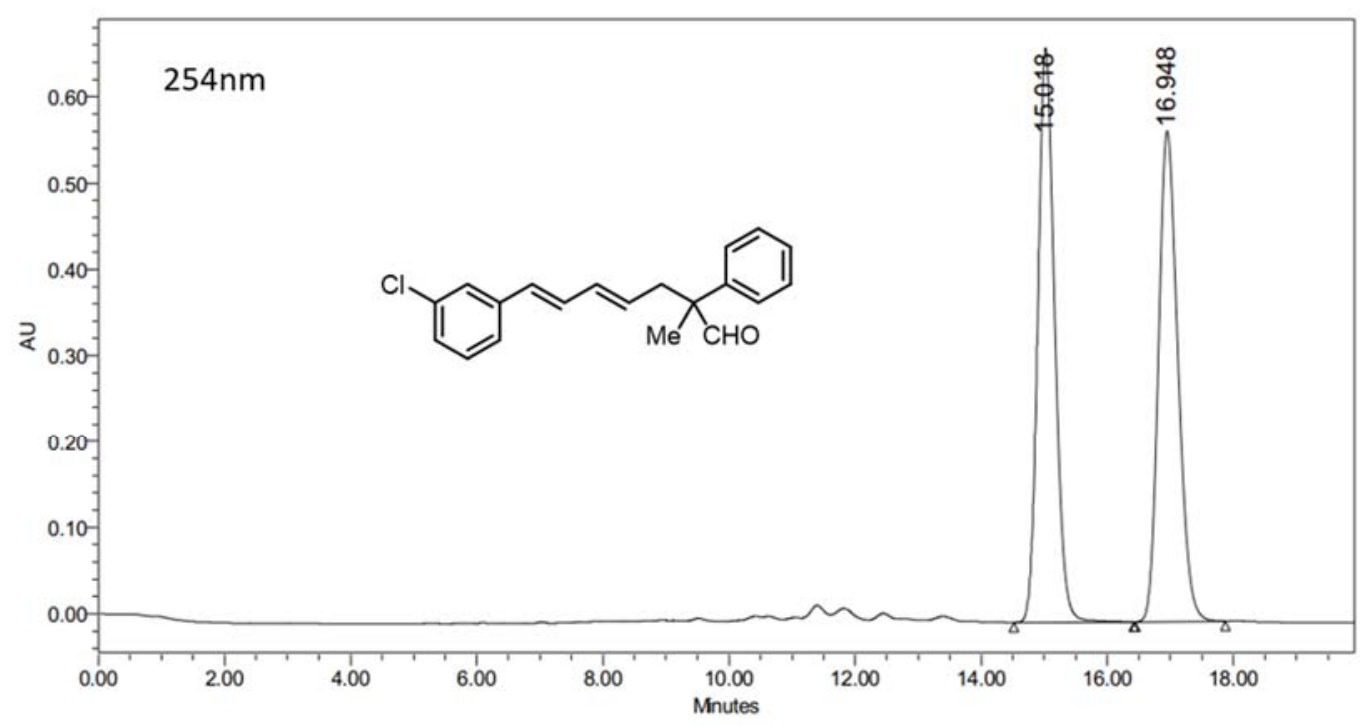

\begin{tabular}{|c|c|c|r|c|}
\hline & RT & Area & $\%$ Area & Height \\
\hline 1 & 15.018 & 12385009 & 50.27 & 666198 \\
\hline 2 & 16.948 & 12254051 & 49.73 & 569429 \\
\hline
\end{tabular}

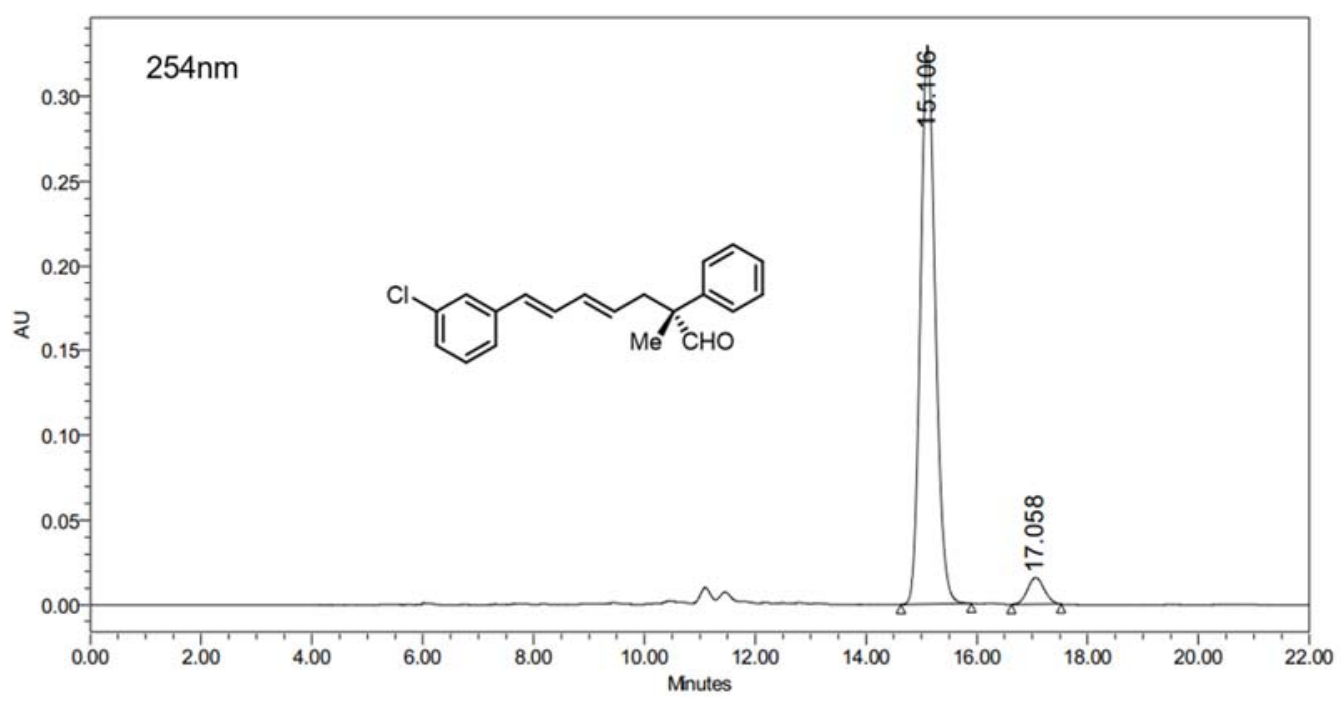

\begin{tabular}{|c|c|c|r|r|}
\hline & RT & Area & $\%$ Area & Height \\
\hline 1 & 15.106 & 6177164 & 94.96 & 329418 \\
\hline 2 & 17.058 & 327923 & 5.04 & 15721 \\
\hline
\end{tabular}


(R,4E,6E)-2-methyl-2-phenyl-7-(m-tolyl)hepta-4,6-dienal (3y)

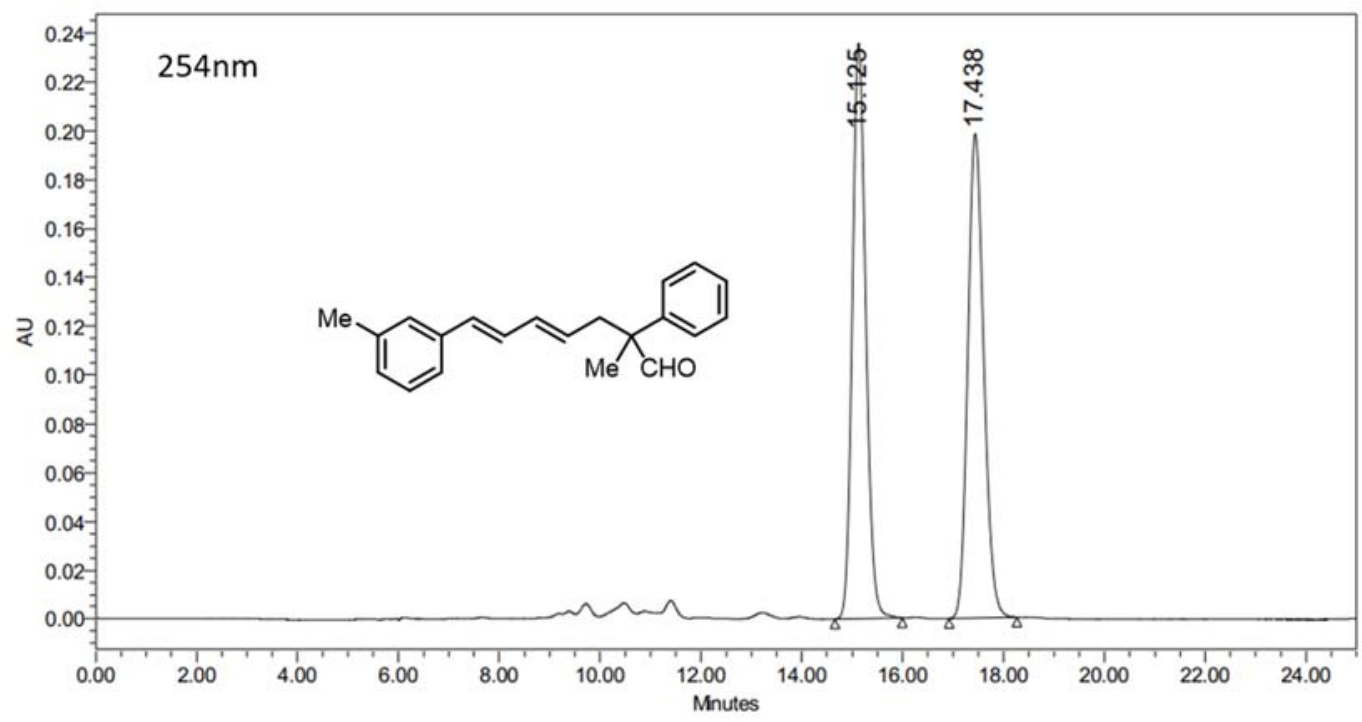

\begin{tabular}{|c|c|c|r|c|}
\hline & RT & Area & $\%$ Area & Height \\
\hline 1 & 15.125 & 4430809 & 50.08 & 235775 \\
\hline 2 & 17.438 & 4416173 & 49.92 & 198920 \\
\hline
\end{tabular}

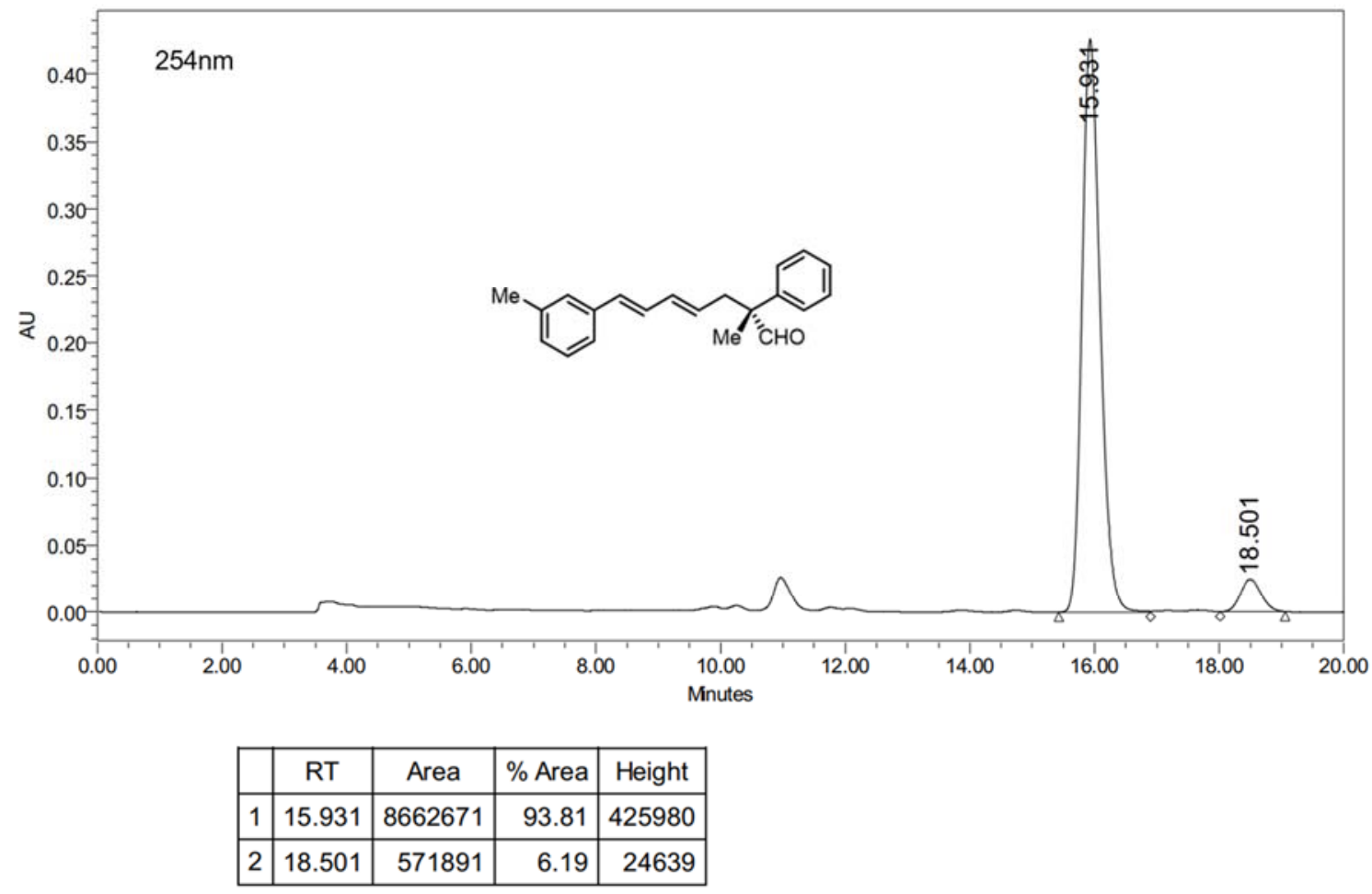



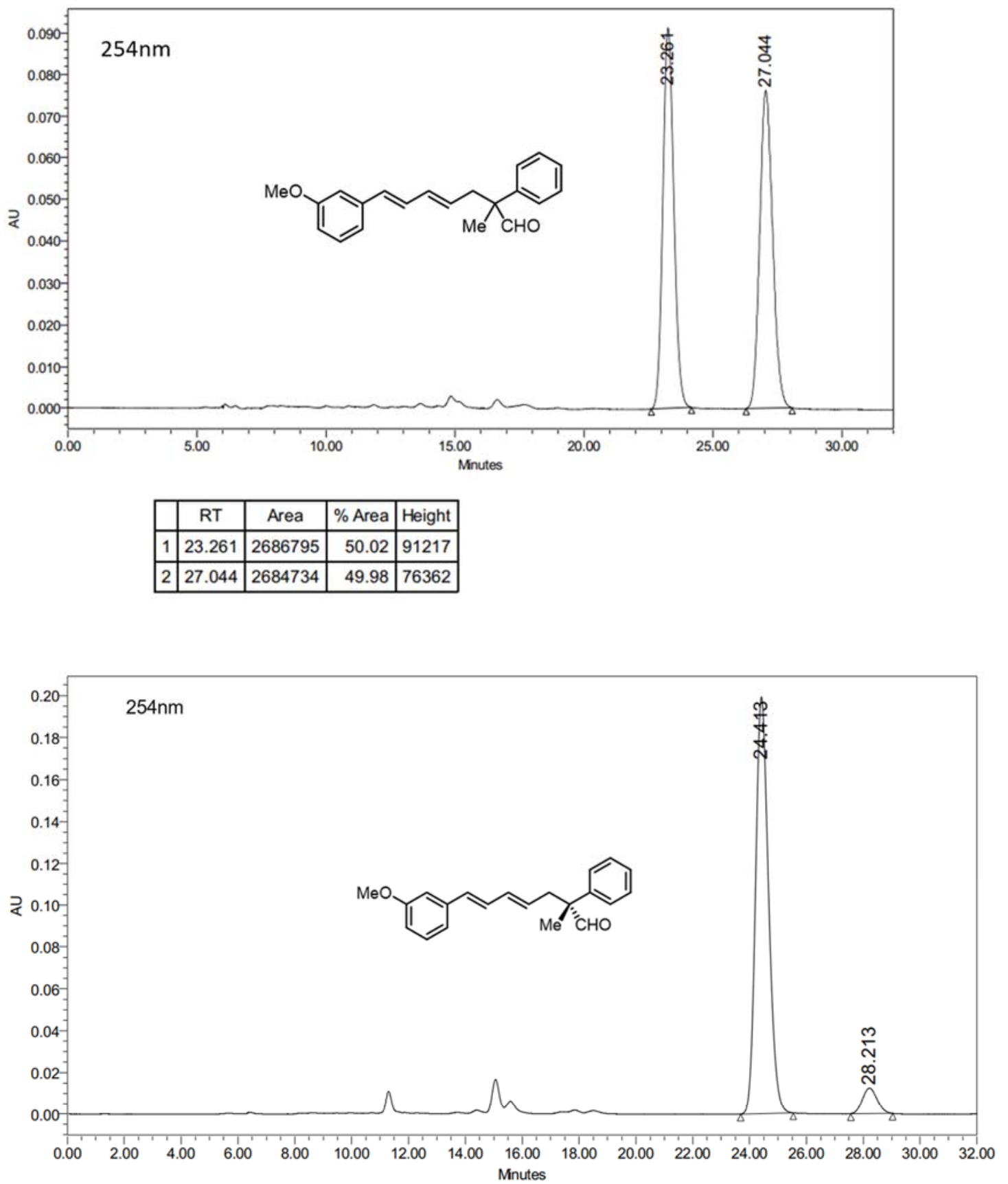

\begin{tabular}{|c|c|c|r|r|}
\hline & RT & Area & $\%$ Area & Height \\
\hline 1 & 24.413 & 6328104 & 93.40 & 199078 \\
\hline 2 & 28.213 & 446968 & 6.60 & 12403 \\
\hline
\end{tabular}


(R,4E,6E)-7-(2-fluorophenyl)-2-methyl-2-phenylhepta-4,6-dienal (3aa)

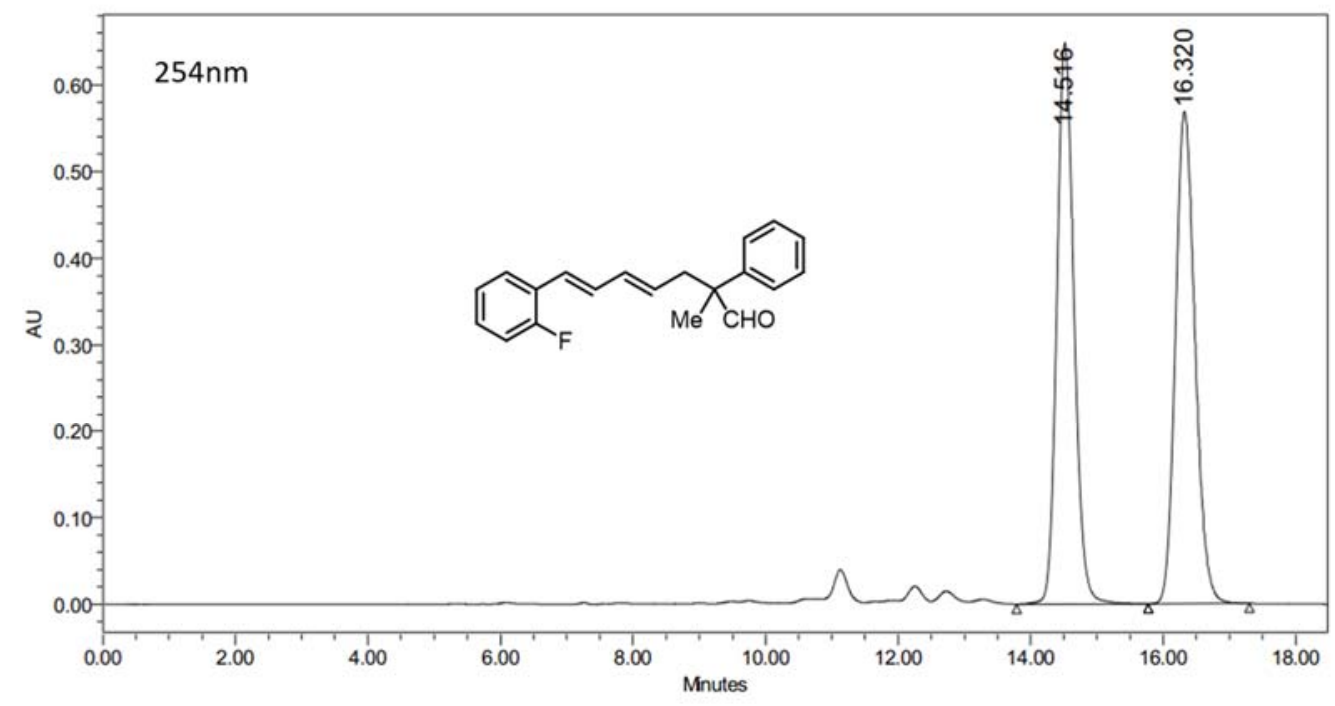

\begin{tabular}{|c|c|c|r|c|}
\hline & RT & Area & $\%$ Area & Height \\
\hline 1 & 14.516 & 11714792 & 50.13 & 648426 \\
\hline 2 & 16.320 & 11655651 & 49.87 & 568613 \\
\hline
\end{tabular}

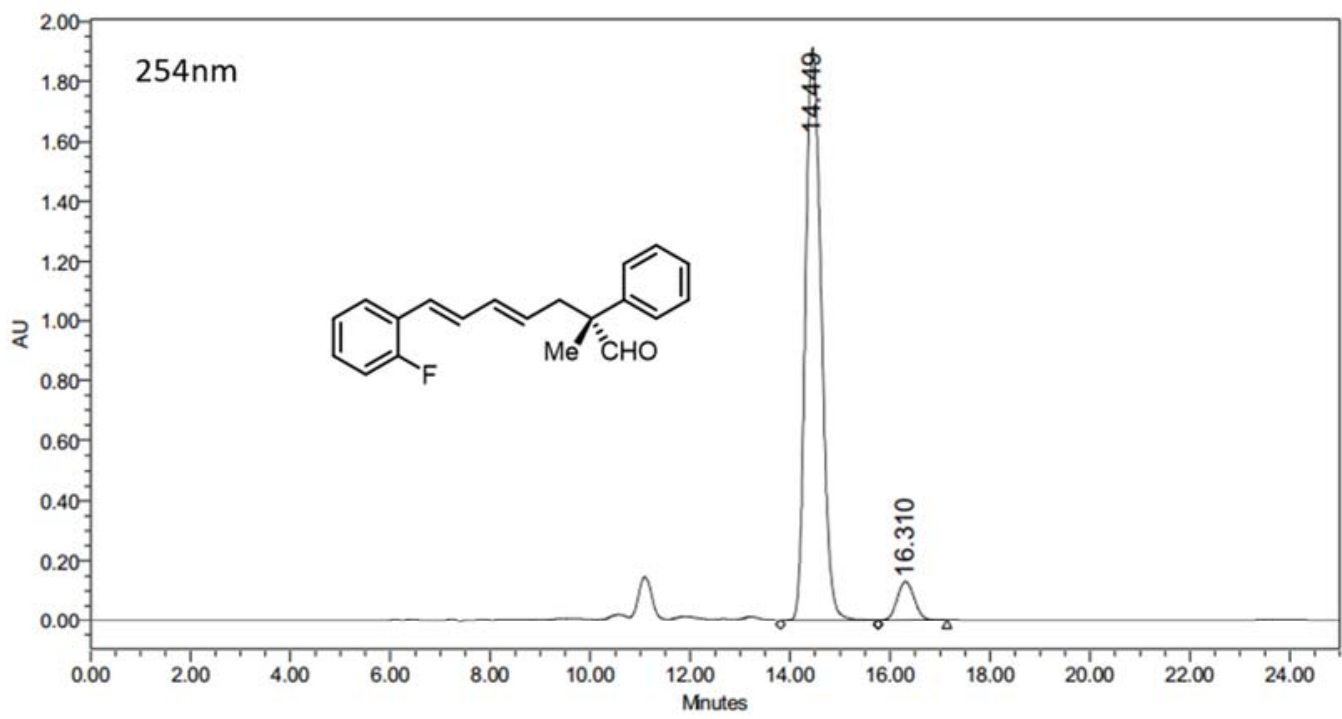

\begin{tabular}{|r|c|r|r|r|}
\hline & RT & \multicolumn{1}{|c|}{ Area } & $\%$ Area & Height \\
\hline 1 & 14.449 & 41630176 & 93.05 & 1911959 \\
\hline 2 & 16.310 & 3111235 & 6.95 & 130400 \\
\hline
\end{tabular}


(R,4E,6E)-7-(2-methoxyphenyl)-2-methyl-2-phenylhepta-4,6-dienal (3ab)

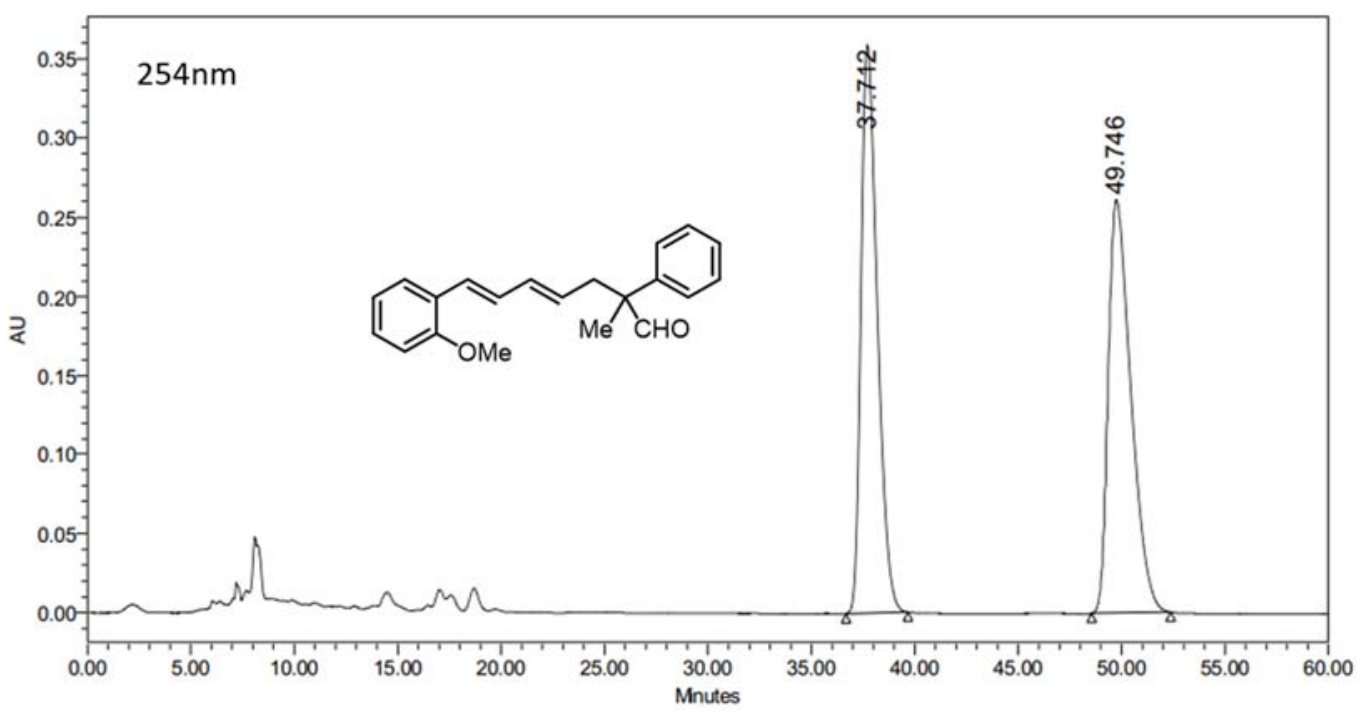

\begin{tabular}{|c|c|c|c|c|}
\hline & RT & Area & $\%$ Area & Height \\
\hline 1 & 37.712 & 19755463 & 49.99 & 358818 \\
\hline 2 & 49.746 & 19763569 & 50.01 & 260942 \\
\hline
\end{tabular}

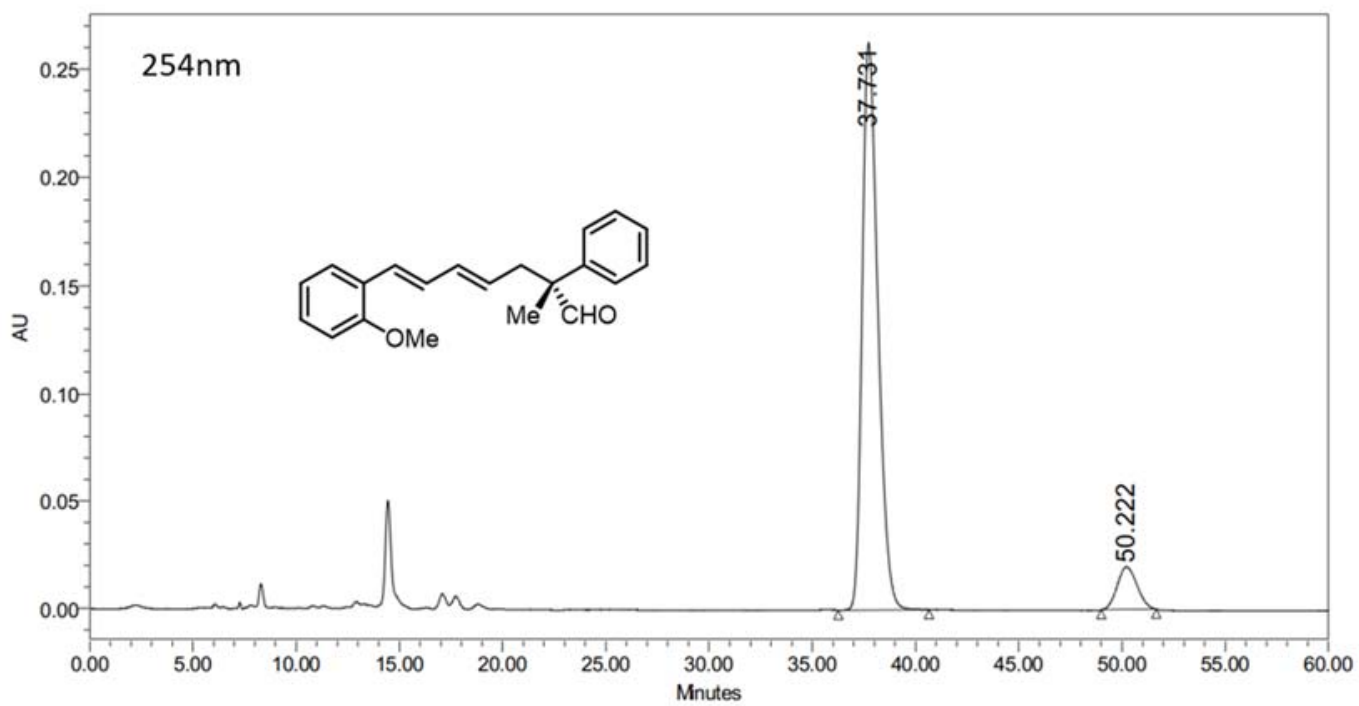

\begin{tabular}{|l|c|c|r|r|}
\hline & RT & Area & $\%$ Area & Height \\
\hline 1 & 37.731 & 13944634 & 91.26 & 262872 \\
\hline 2 & 50.222 & 1336013 & 8.74 & 19855 \\
\hline
\end{tabular}




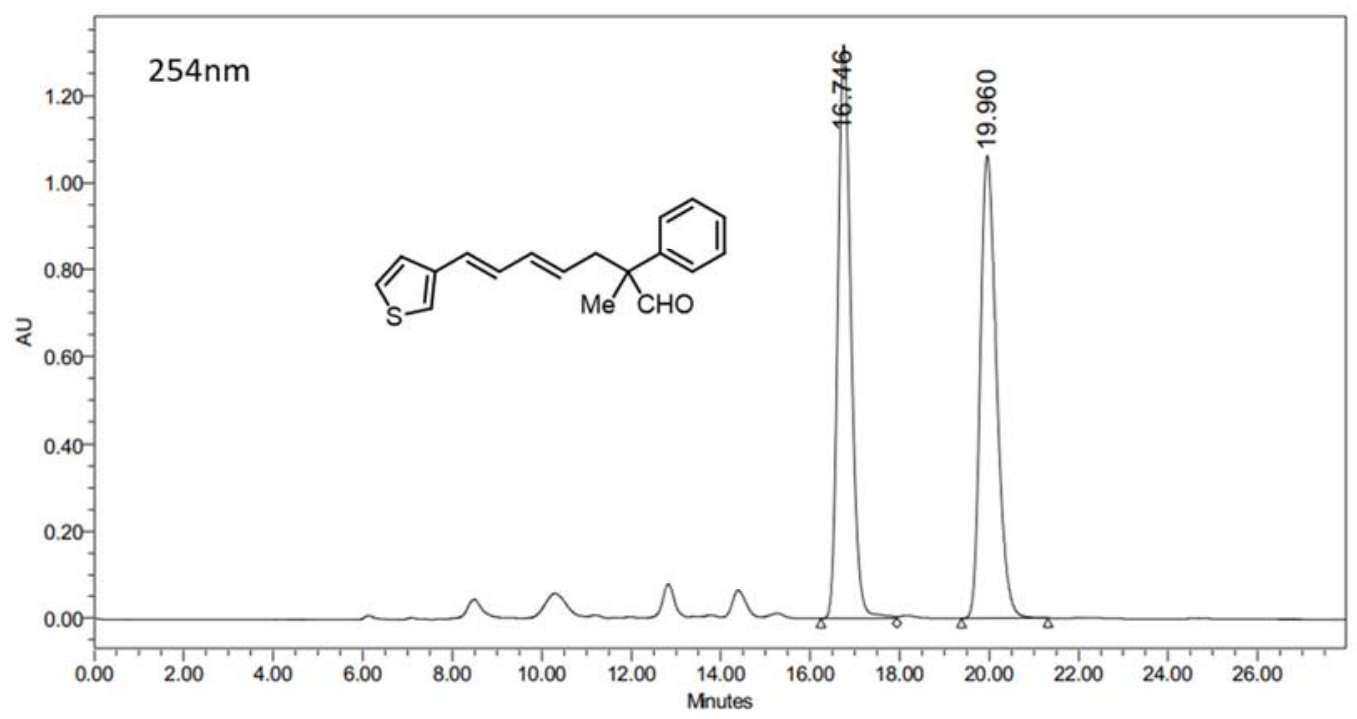

\begin{tabular}{|c|c|c|r|c|}
\hline & RT & Area & $\%$ Area & Height \\
\hline 1 & 16.746 & 27028257 & 50.17 & 1316364 \\
\hline 2 & 19.960 & 26845752 & 49.83 & 1064320 \\
\hline
\end{tabular}

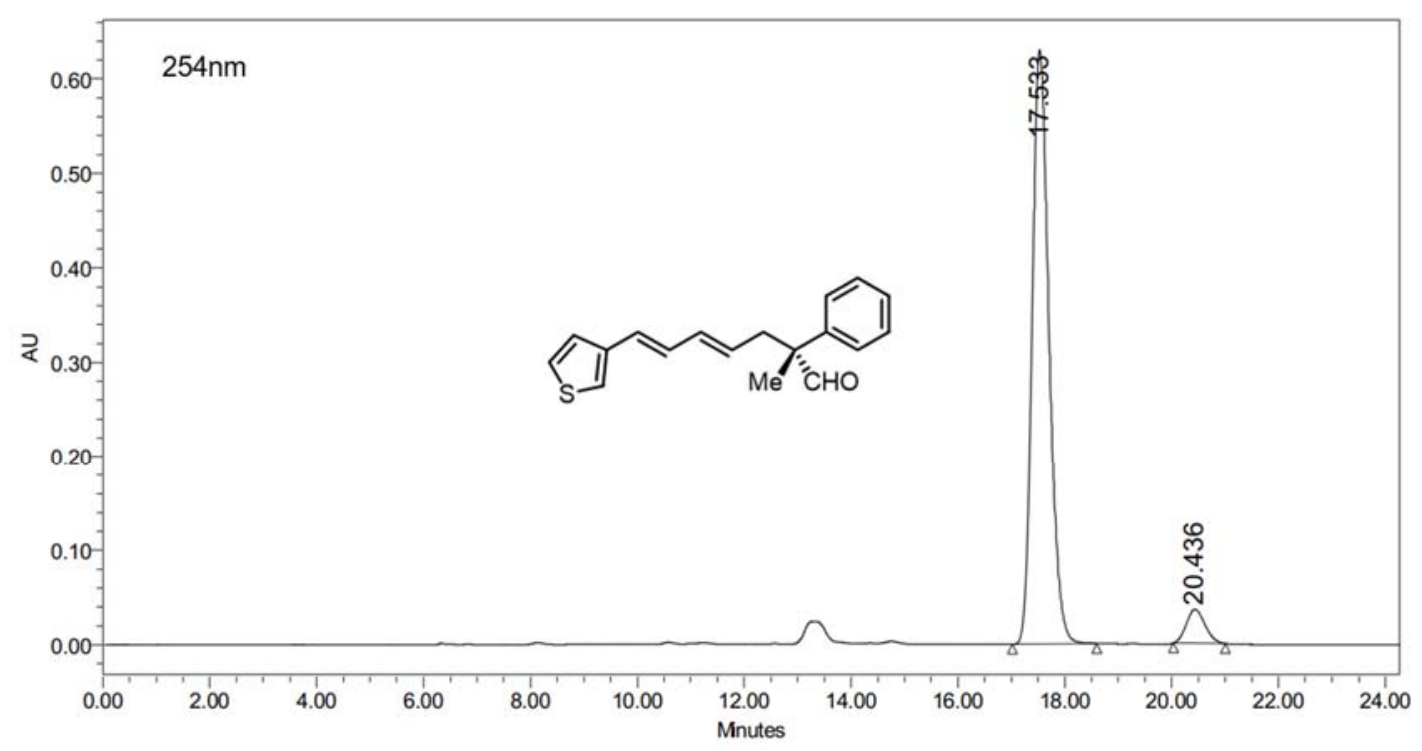

\begin{tabular}{|l|c|r|r|r|}
\hline & RT & \multicolumn{1}{|c|}{ Area } & \% Area & Height \\
\hline 1 & 17.533 & 13702395 & 93.96 & 630049 \\
\hline 2 & 20.436 & 880479 & 6.04 & 35911 \\
\hline
\end{tabular}


(2S,4R)-4-methyl-4-phenyl-2-((E)-prop-1-en-1-yl)tetrahydrofuran (4-major)

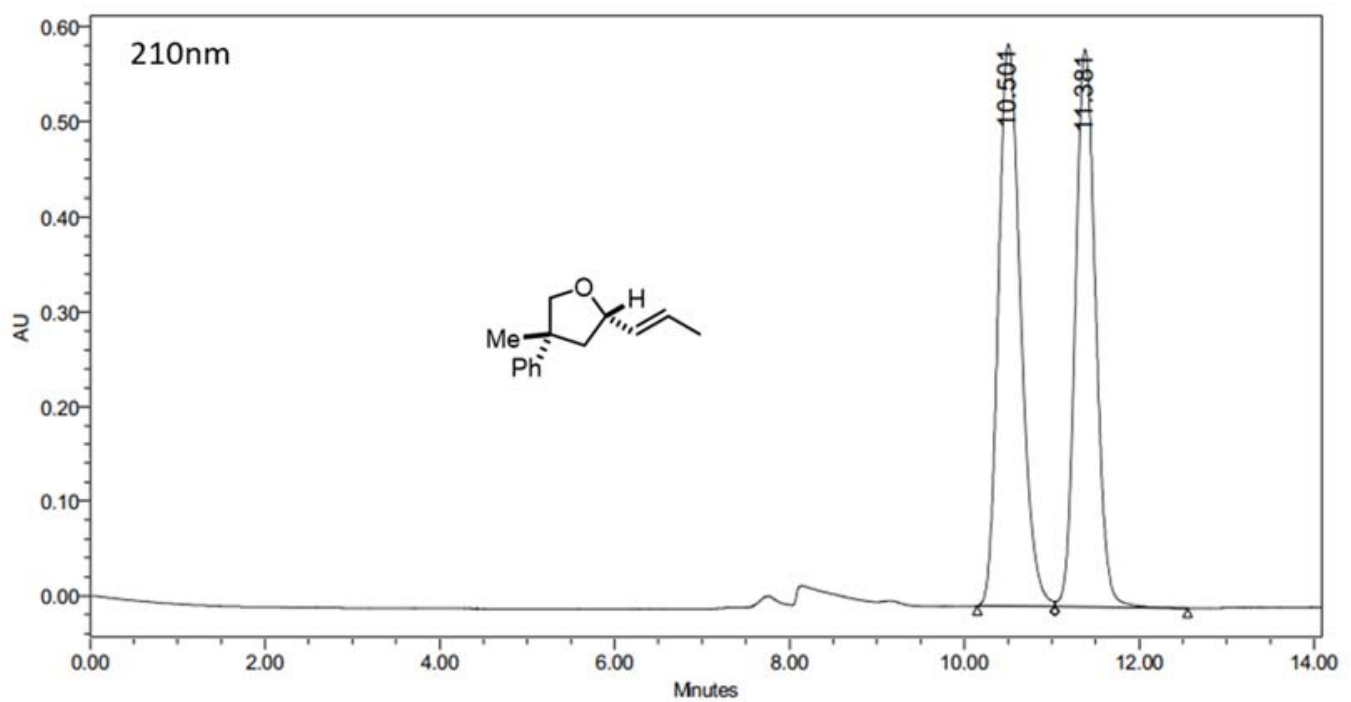

\begin{tabular}{|c|c|c|r|c|}
\hline & RT & Area & $\%$ Area & Height \\
\hline 1 & 10.501 & 10462089 & 52.62 & 592502 \\
\hline 2 & 11.381 & 9420285 & 47.38 & 587911 \\
\hline
\end{tabular}

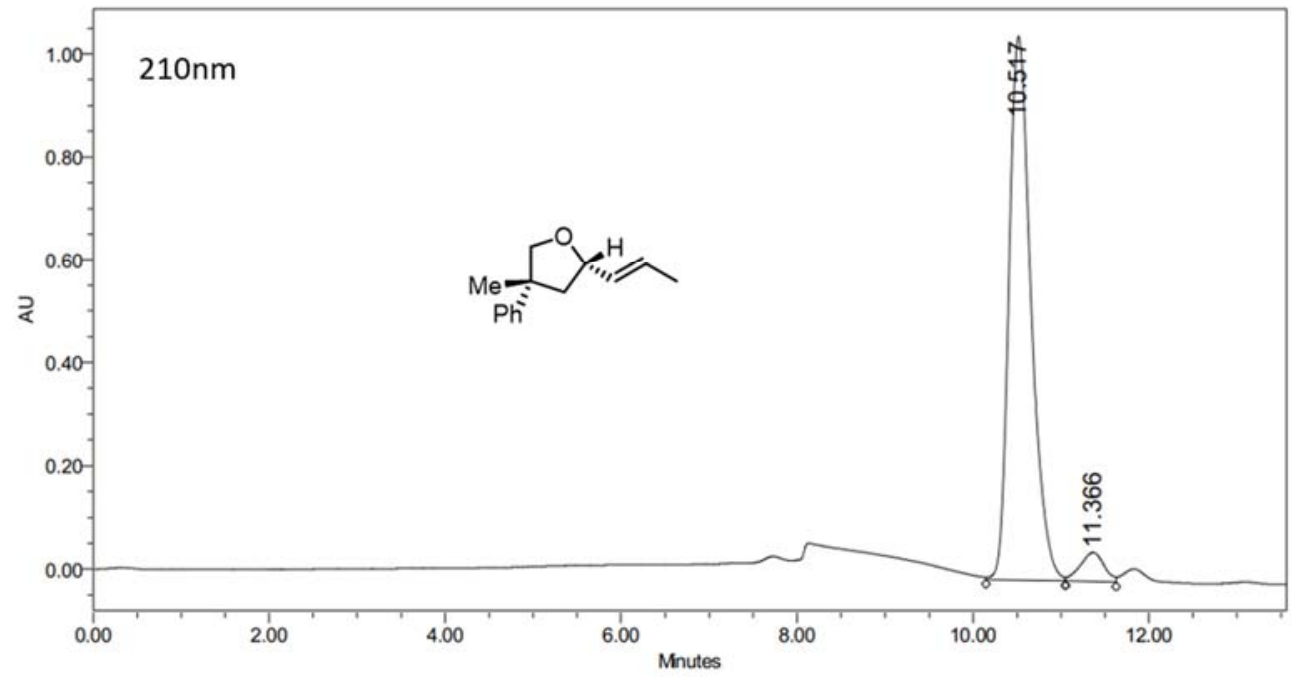

\begin{tabular}{|c|c|c|r|r|}
\hline & RT & Area & \% Area & Height \\
\hline 1 & 10.517 & 18440394 & 94.57 & 1055605 \\
\hline 2 & 11.366 & 1058903 & 5.43 & 56267 \\
\hline
\end{tabular}


(2R,4R)-4-methyl-4-phenyl-2-((E)-prop-1-en-1-yl)tetrahydrofuran (4-minor)

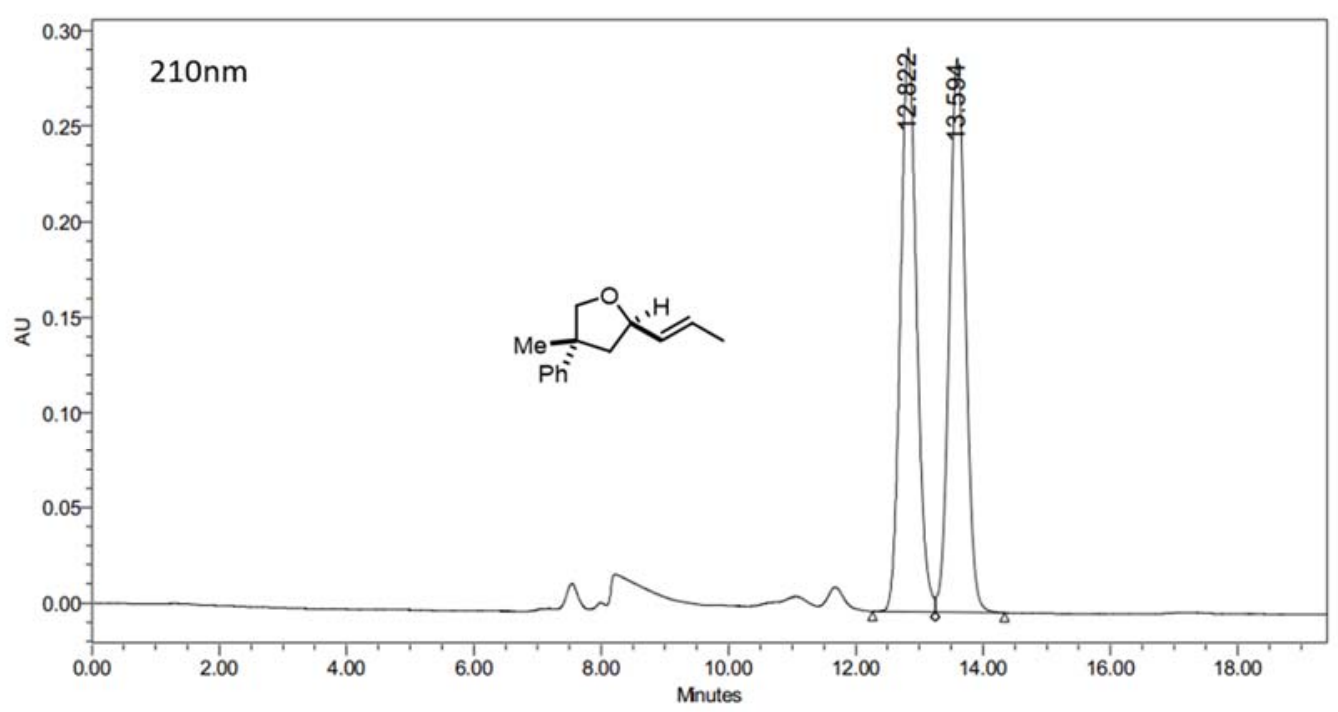

\begin{tabular}{|c|c|c|r|c|}
\hline & RT & Area & \% Area & Height \\
\hline 1 & 12.822 & 5219255 & 50.55 & 295383 \\
\hline 2 & 13.594 & 5106422 & 49.45 & 290333 \\
\hline
\end{tabular}

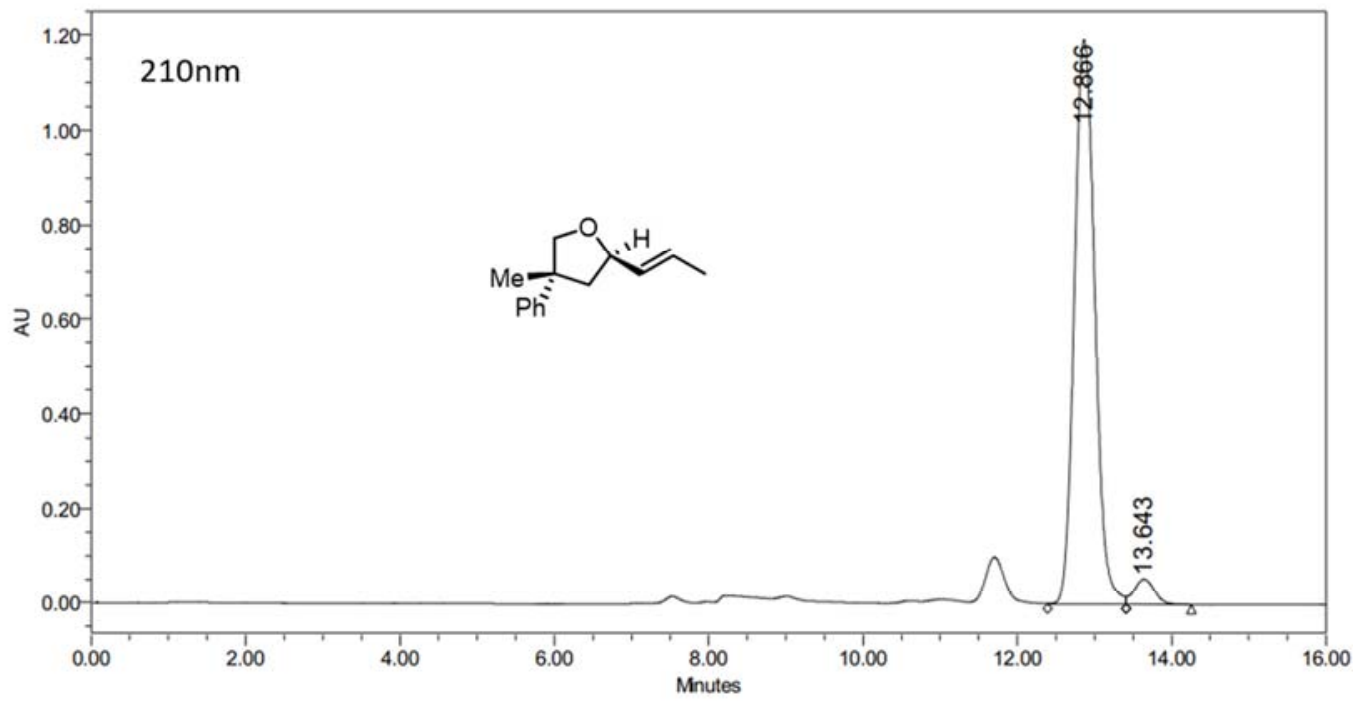

\begin{tabular}{|r|c|r|r|r|}
\hline & RT & \multicolumn{1}{|c|}{ Area } & \% Area & \multicolumn{1}{c|}{ Height } \\
\hline 1 & 12.866 & 21945038 & 95.68 & 1193019 \\
\hline 2 & 13.643 & 989890 & 4.32 & 54203 \\
\hline
\end{tabular}


(R,E)-N-benzyl-2-methyl-2-phenylhepta-4,6-dien-1-amine (5)

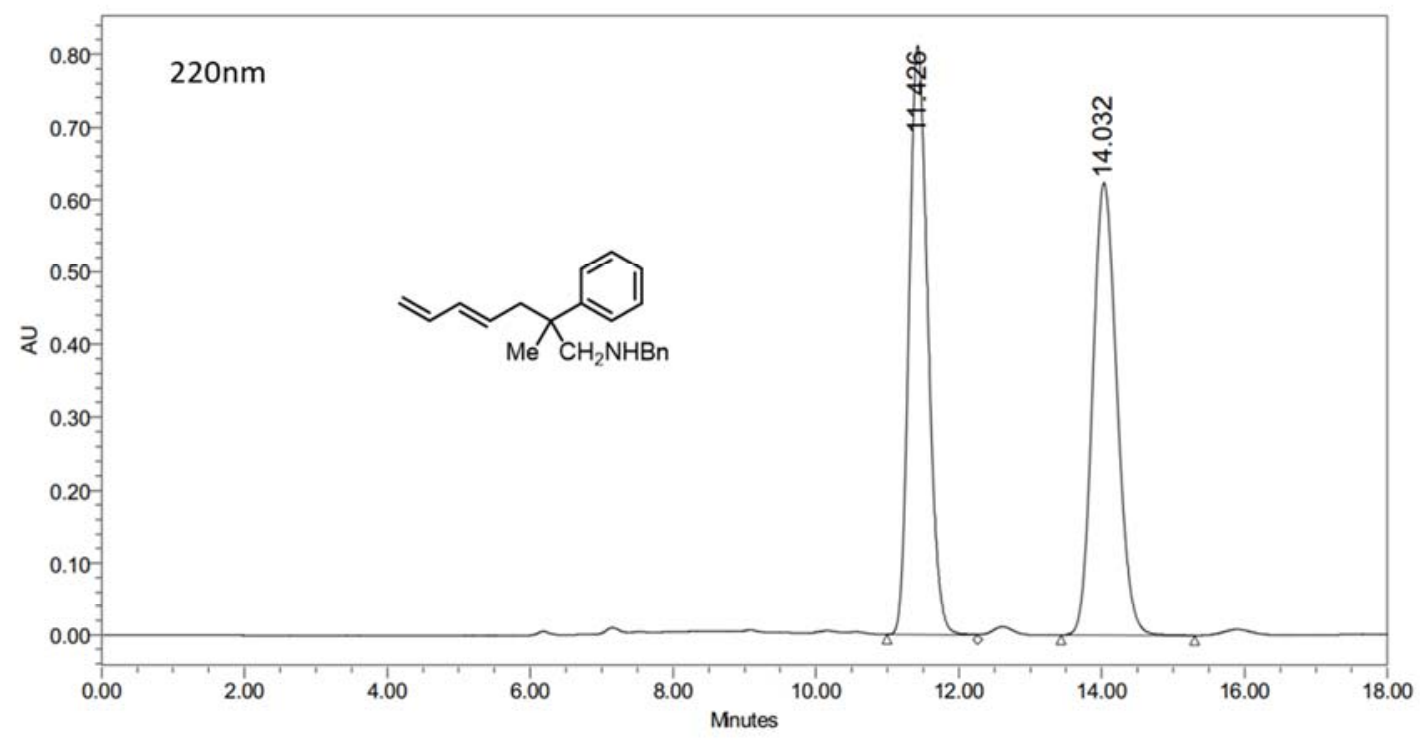

\begin{tabular}{|c|c|c|r|c|}
\hline & RT & Area & $\%$ Area & Height \\
\hline 1 & 11.426 & 14829389 & 50.02 & 811819 \\
\hline 2 & 14.032 & 14815156 & 49.98 & 624030 \\
\hline
\end{tabular}

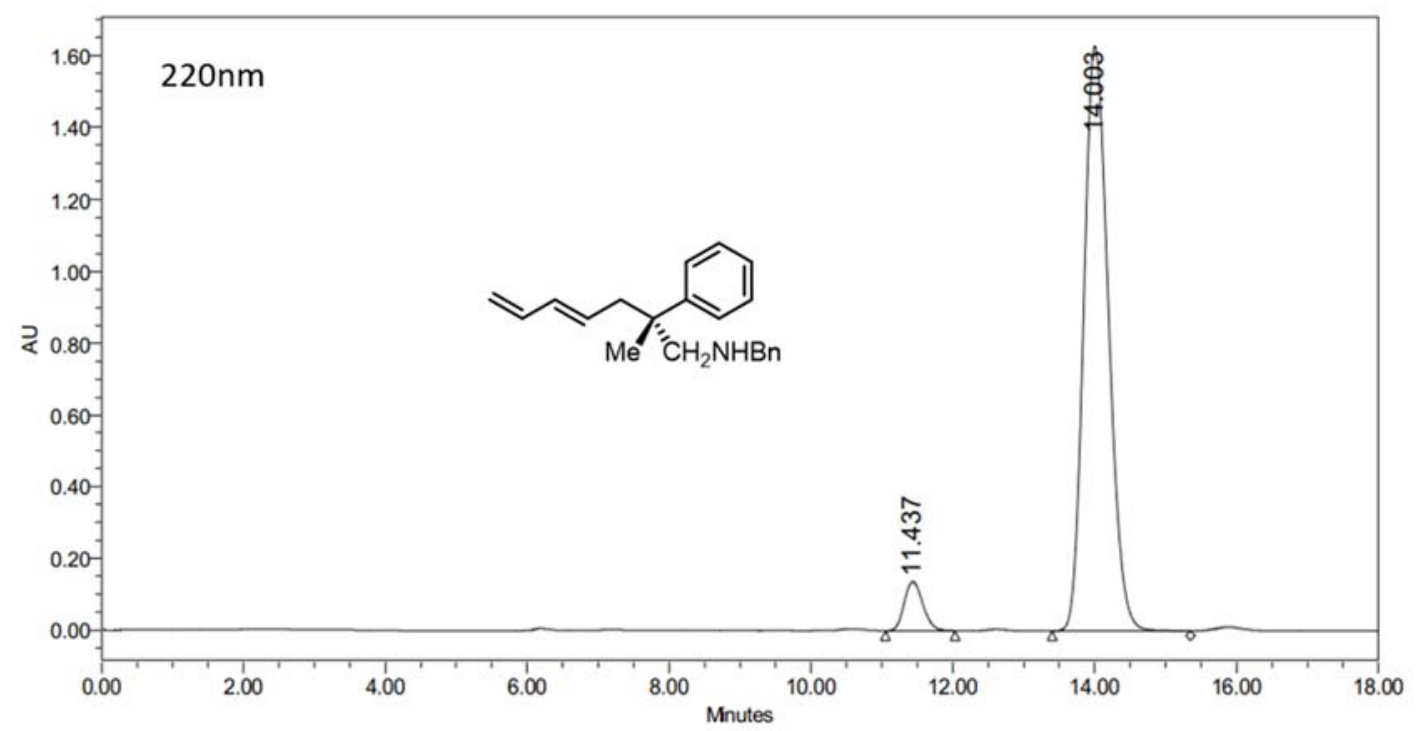

\begin{tabular}{|c|c|r|r|c|}
\hline & RT & Area & $\%$ Area & Height \\
\hline 1 & 11.437 & 2495359 & 5.95 & 136969 \\
\hline 2 & 14.003 & 39473422 & 94.05 & 1627432 \\
\hline
\end{tabular}


(S,E)-(3-methylocta-5,7-dien-1-yn-3-yl)benzene (6)

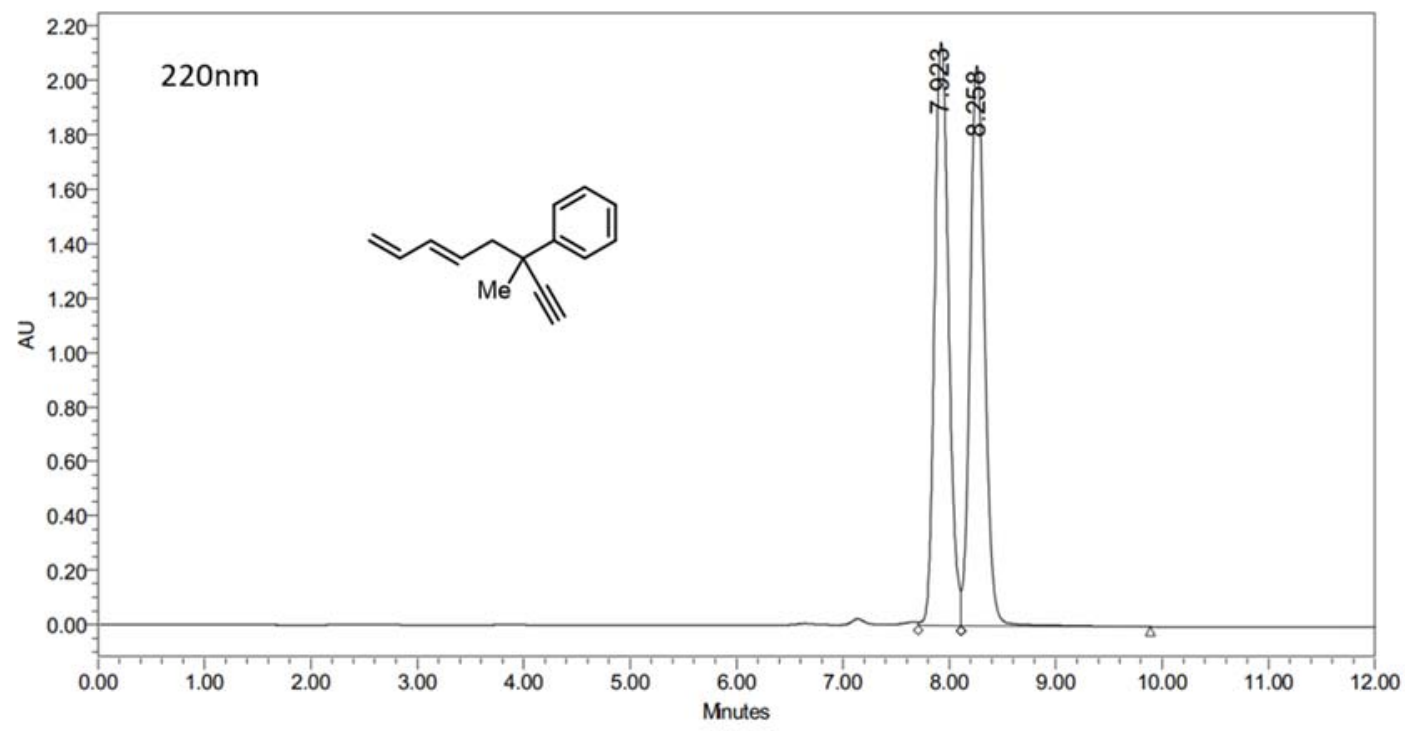

\begin{tabular}{|c|c|c|r|c|}
\hline & RT & Area & $\%$ Area & Height \\
\hline 1 & 7.923 & 19584142 & 49.87 & 2142013 \\
\hline 2 & 8.258 & 19687661 & 50.13 & 2057377 \\
\hline
\end{tabular}

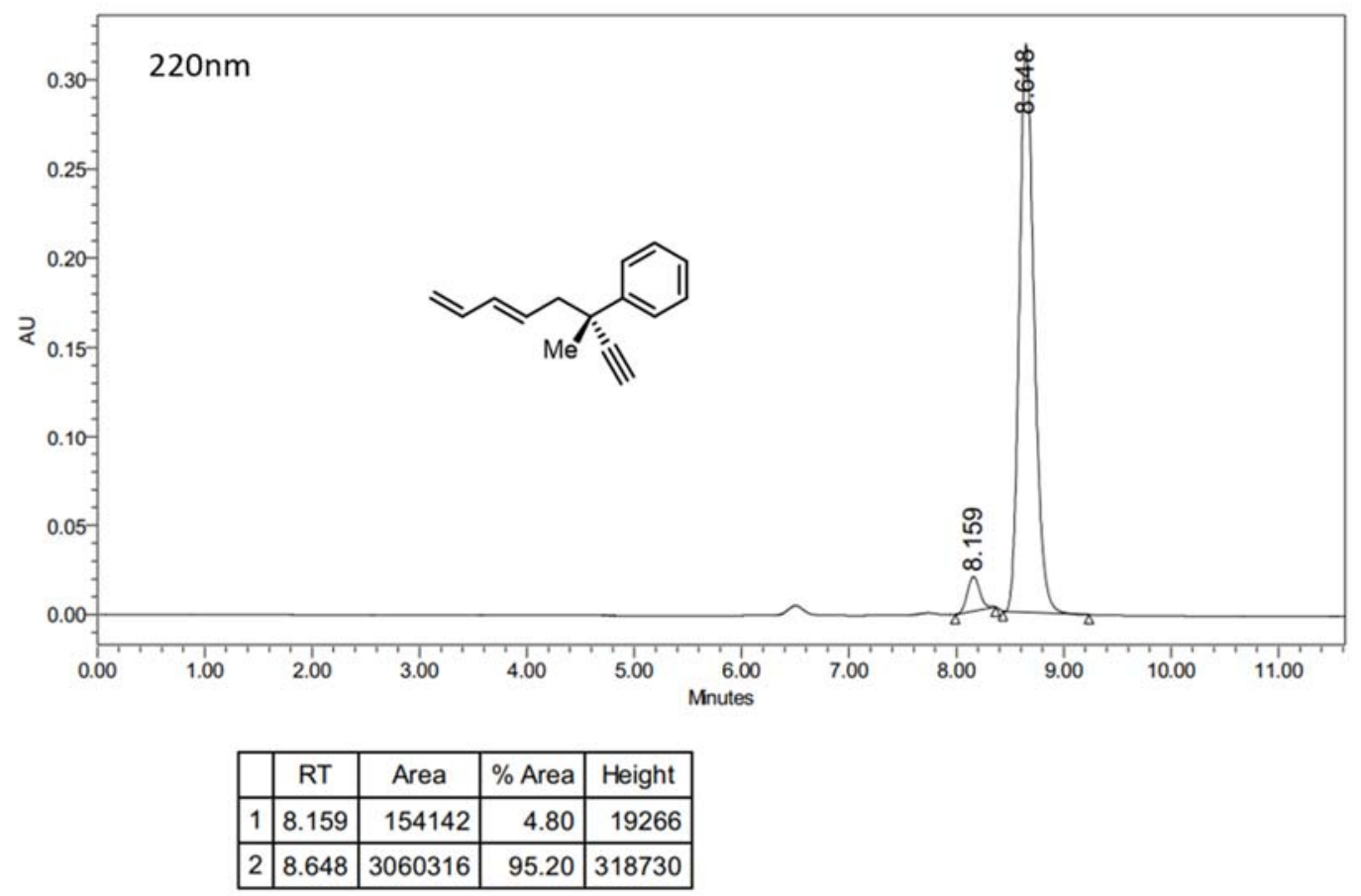

